Tipos de homotopia dos grupos de gauge dos fibrados linhas quaterniônicos sobre esferas 


\title{
Tipos de homotopia dos grupos de gauge dos fibrados linhas quaterniônicos sobre esferas ${ }^{1}$
}

\author{
Mario Henrique Andrade Claudio \\ Orientador: Prof. Dr. Mauro Flavio Spreafico
}

Tese apresentada ao Instituto de Ciências Matemáticas e de Computação da Universidade de São Paulo, como parte dos requisitos para obtenção do título de Doutor em Ciências Área: Matemática.

USP - São Carlos

Abril/2008

\footnotetext{
${ }^{1}$ Este trabalho teve suporte financeiro da Capes
} 
Aos meus pais,

Luiz Antonio e Ursulina, com carinho... 


\section{Agradecimentos}

Primeiramente a Deus, por me iluminar e me dar força nos momentos mais difíceis, me guiando ao final de mais uma etapa da minha vida.

Ao meu orientador, Prof. Mauro Flavio Spreafico, pela orientação, me ajudando nos momentos em que precisei. Agradeço pela paciência, incentivo e pelo exemplo de profissional que mostrou ser durante esses anos.

Aos meus pais e minhas irmãs, que sempre me incentivaram a buscar novos caminhos e idéias, não me deixando esquecer que o ser humano é um conjunto de seus conhecimentos e das ações que pratica. Obrigado por todo amor que dedicaram a mim. Amo vocês!

A toda minha família, que sempre acreditou em mim e por todo carinho e amizade.

Ao Prof. Oziride Manzoli Neto, pela amizade em todos esses anos de minha vida acadêmica e pelas sugestões no trabalho. Agradeço, também a todos os professores que participaram de minha formação pela amizade e incentivo constante.

Aos meus amigos, que depois de todos esses anos a quantidade é grande para listar todos aqui, mas no coração todos estão bem guardados. Agradeço pela ajuda, vinda em momentos em que vocês nem imaginavam, pelas risadas, pelas conversas e por todos os outros bons momentos que passamos juntos.

Aos funcionários do ICMC e a todos os que colaboraram para a realização deste trabalho.

À CAPES pelo suporte financeiro.

Muito obrigado! 


\section{Abstract}

Let $p$ be a principal $S^{3}$-bundle over a sphere $S^{n}$, with $n \geq 4$. The subject of this work is to calculate the homotopy type of the gauge group $\mathcal{G}_{p}$ of these bundles $p$, extending the result determined by A. Kono [25] when $n=4$. We present explicit formulas for the boundary operator in the homotopy exact sequence associated with the evaluation map ev $: m\left(S^{n}, B S^{3}\right) \rightarrow B S^{3}$, translating that problem into calculations involving homotopy groups of sphere. We calculate all the classical cases, namely those that can be dealt with using the information in the book of $\mathrm{H}$. Toda [46], determining the homotopy type of the gauge group of these bundles for each $n \leq 25$. 


\section{Resumo}

Seja $p$ um $S^{3}$-fibrado principal sobre uma esfera $S^{n}$, com $n \geq 4$. O objetivo deste trabalho é calcular os tipos de homotopia do grupo de gauge $\mathcal{G}_{p}$ desses fibrados $p$, estendendo o resultado determinado por A. Kono [25] quando $n=4$. Apresentamos fórmulas explícitas para o operador bordo na seqüência exata de homotopia associada com a aplicação avaliação ev $: m\left(S^{n}, B S^{3}\right) \rightarrow B S^{3}$, traduzindo o problema nos cálculos envolvendo grupos de homotopia de esferas. Calculamos todos os casos clássicos, ou seja, aqueles que podem ser avaliados usando as informações encontradas no livro de H. Toda [46], determinando o tipo de homotopia do grupo de gauge desses fibrados para cada $n \leq 25$. 


\section{Sumário}

Introdução 1

1 Preliminares 3

1.1 O espaço de aplicações contínuas . . . . . . . . . . . . . . . . 3

1.2 Fibrações e co-fibrações . . . . . . . . . . . . . . . . . . . . . 6

1.3 Seqüências exatas de homotopia . . . . . . . . . . . . . . . . . . . 9

1.4 Alguns lemas . . . . . . . . . . . . . . . . . . . . . . . 11

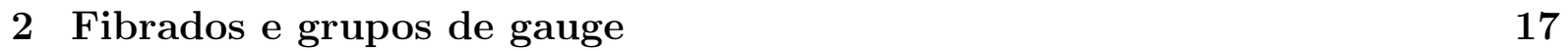

2.1 Grupos topológicos e ações . . . . . . . . . . . . . . . . . . . . . 17

2.2 Fibrados . . . . . . . . . . . . . . . . . . . 22

2.2.1 Definição global de um fibrado principal . . . . . . . . . . . . . . 22

2.2.2 Fibrados associados . . . . . . . . . . . . . . . . . . 22

2.2.3 Definição local de fibrados . . . . . . . . . . . . . . . . . . . 23

2.2.4 Equivalência de fibrados . . . . . . . . . . . . . . . 25

2.2 .5 Seções e trivialidade . . . . . . . . . . . . . . . . . . . 26

2.2.6 Fibrado pull back induzido . . . . . . . . . . . . . . . . . . 26

2.2.7 Somando fibrados . . . . . . . . . . . . . . . . . 27

2.2 .8 Exemplos de fibrados . . . . . . . . . . . . . . . . . . . . . 28

2.3 Classificação de homotopia de $G$-fibrados principais . . . . . . . . . . . . . 29

2.4 Grupos de gauge . . . . . . . . . . . . . . . . . . . . . . . . . . . . . . . . . . . . .

2.4.1 A homotopia do grupo de gauge . . . . . . . . . . . . . 35

2.5 O espaço dos laços sobre o espaço classificante de um grupo topológico . . 38

2.6 Apêndice do capítulo $2 \ldots \ldots \ldots$. . . . . . . . . . . . . 42

3 Métodos de composição dos grupos de homotopia de esferas 47

3.1 O produto de Whitehead . . . . . . . . . . . . . . . . . . . . . . . . . 48

3.2 A seqüência exata $\Sigma H P \ldots \ldots \ldots \ldots$. . . . . . . . . . . . . . . . . . . . . . . . 59

3.3 As componentes primárias de $\pi_{2+k}\left(S^{2}\right)$ e $\pi_{3+k}\left(S^{3}\right)$ com $1 \leq k \leq 19 \ldots$. . . 53

4 Tipo de homotopia dos grupos de gauge dos $S^{3}$-fibrados sobre esferas $\quad \mathbf{6 7}$

4.1 A imagem do homomorfismo $e v_{j} \ldots \ldots . \ldots . . \ldots 69$

4.2 Alguns resultados sobre os grupos de homotopia das fibras . . . . . . . . . 75

4.3 Os tipos de homotopia dos grupos de gauge . . . . . . . . . . . . . . 79 
4.3.1 Os casos $5 \leq n \leq 12, n=16,17,18,23,24,25 \ldots \ldots$. . . . . 79

4.3.2 Os casos $n=14,19,20,22 \ldots \ldots \ldots \ldots$. . . . . . . . . . . . . . . . . . . . . . 84

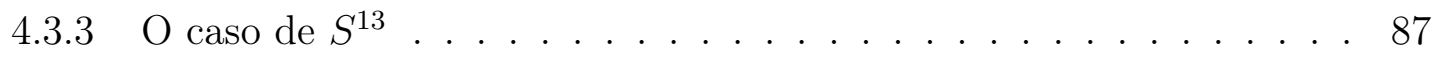

4.3 .4 O caso de $S^{21} \ldots \ldots \ldots 119$

4.4 Apêndice do capítulo $4 \ldots \ldots$. . . . . . . . . . . . . 130

Trocando a esfera $S^{n}$ pelo espaço projetivo quaterniônico 135

$\begin{array}{ll}\text { Referências Bibliográficas } & 141\end{array}$

$\begin{array}{ll}\text { Tabela de símbolos } & 145\end{array}$

$\begin{array}{ll}\text { Îndice Remissivo } & 146\end{array}$ 


\section{Introdução}

Dado um $G$-fibrado principal, $p: P \rightarrow B$, sobre um complexo finito $B$, onde $G$ é um grupo de Lie compacto, definimos o grupo de gauge $\mathcal{G}_{p}$ de $p$ como o grupo das aplicações $G$-eqüivariantes de $P$ cobrindo a identidade. Quando $B$ e $G$ são conexos, um estudo foi feito por M. C. Crabb e W. Sutherland em [9], onde provaram que o número de tipos de homotopia de $\mathcal{G}_{p}$ é finito. Além disso, resultados explícitos foram dados por A. Kono e H. Hamanaka, que estudaram em uma série de trabalhos o caso em que $B=S^{4}$ e $G=S U(n)$. Esses fibrados $P_{n, k}$ estão classificados pela classe de Chern $c_{2}\left(P_{n, k}\right)=k$, e foi provado em [25] que $\mathcal{G}_{P_{2, k}} \sim \mathcal{G}_{P_{2, k^{\prime}}}$ se, e somente se, $(12, k)=\left(12, k^{\prime}\right)$ (aqui $(m, n)$ denota o máximo divisor comum entre $n$ e $m$ ), e existem seis tipos de homotopia. Foi provado em [14] que $\mathcal{G}_{P_{3, k}} \sim \mathcal{G}_{P_{3, k^{\prime}}}$ se, e somente se, $(24, k)=\left(24, k^{\prime}\right)$. Recentemente, os mesmos autores estudaram o caso $B=S^{6}$ e $G=S U(3)$. Esses fibrados estão classificados pela classe de Chern $c_{3}\left(P_{k}\right)=k$, e provaram em [15] que $\mathcal{G}_{P_{k}} \sim \mathcal{G}_{P_{k^{\prime}}}$ se, e somente se, $(120, k)=\left(120, k^{\prime}\right)$.

Seguindo essa linha de investigação, estudamos o caso dos $S U(2)$-fibrados principais sobre uma esfera $S^{n}$. Nosso objetivo consiste em generalizar o método introduzido em [25] com $n=4$ para um $n$ maior. Em particular, apresentamos fórmulas explícitas para o operador bordo na seqüência exata de homotopia associada com a aplicação avaliação ev $: m\left(S^{n}, B S^{3}\right) \rightarrow B S^{3}$, traduzindo o problema para cálculos envolvendo grupos de homotopia de esferas, e resolvendo até o ponto em que as informações sobre esses grupos estejam disponíveis. Apresentamos o resultado para todos os casos clássicos, ou seja, aqueles que podem ser avaliados usando as informações encontradas no livro de H. Toda [46].

O trabalho será apresentado da seguinte forma:

No Capítulo 1, apresentaremos todo o material básico necessário no desenvolvimento do trabalho, recordando rapidamente os seguintes itens: o espaço das aplicações contínuas, pull back e push out, fibrações e co-fibrações, seqüências exatas de homotopia e alguns lemas sobre equivalência de homotopia.

No Capítulo 2, recordamos as definições (global e local) de fibrados, fibrados principais, fibrados associados, fibrado pull back induzido, somando fibrados e também vemos a classificação de homotopia de $G$-fibrados principais, a definição dos grupos de gauge e sua homotopia, e ainda o espaço dos laços sobre o espaço classificante de um grupo topológico.

No Capítulo 3, fazemos um pequeno resumo dos resultados apresentados no livro de H. Toda [46], vendo a definição de decomposição secundária, a seqüência exata $\Sigma H P$ e 
as componentes primárias dos grupos de homotopia das esferas, $S^{2}$ e $S^{3}$ em especial, e também uma breve apresentação dos resultados sobre o produto de Whitehead.

Finalmente, no Capítulo 4, calculamos os tipos de homotopia dos grupos de gauge dos fibrados linhas quaterniônicos sobre esferas, iniciando com o cálculo explícito do homomorfismo avaliação $e v_{j}$, seguido da seção em que vemos alguns resultados sobre os grupos de homotopia das fibras. Em duas outras seções, temos também os casos de $S^{13}$ e $S^{21}$, que por não apresentarem o mesmo comportamento dos outros casos, merecem uma atenção especial e são trabalhados com mais detalhes. Fechamos o capítulo com uma proposição reunindo todo os resultados obtidos (com uma tabela contendo os grupos de homotopia dos grupos envolvidos).

Finalizamos o trabalho discutindo a idéia de se considerar o espaço projetivo quaterniônico, $\mathbb{H} P^{2}$, ao invés da esfera $S^{n}$, como uma proposta de trabalho futuro. 


\section{Capítulo}

\section{Preliminares}

Neste capítulo, apresentaremos algumas definições e resultados básicos sobre teoria de homotopia, os quais serão necessários para o desenvolvimento dos resultados dos capítulos posteriores. Uma discussão mais completa desses resultados, incluindo provas e aplicações, pode ser encotrada em várias referências, dentre elas [10], [23],[37] e [47].

\subsection{O espaço de aplicações contínuas}

Seja Top a categoria dos espaços topológicos e das funções contínuas. Os objetos e morfismos de tal categoria serão chamados de espaços e aplicações contínuas, respectivamente. Do mesmo modo, Topo representa a categoria dos espaços baseados e aplicações contínuas baseadas. Um espaço baseado será denotado junto com seu ponto base como um par, $\left(X, x_{0}\right)$. Em ambas as categorias, o símbolo $\cong$ denota homeomorfismo.

Dados $X, Y \in T o p$, seja $m(X, Y)$ o espaço das funções de todas as aplicações contínuas $f: X \rightarrow Y$ com a topologia compacto-aberta. Recordemos que para $X, Y \in T o p$, os conjuntos $W_{K, U}=\{f \in m(X, Y) \mid f(K) \subset U\}$, onde $K \subset X$ é compacto e $U \subset Y$ é aberto, formam uma sub-base para a topologia compacto-aberta de $m(X, Y)$.

Os dois próximos resultados podem ser encontrados em [37].

Teorema 1.1.1. Seja $X \in$ Top um espaço de Haudorff localmente compacto. Então, se $m(X, Y)$ possui a topologia compacto-aberta, a função

$$
e v: m(X, Y) \times X \rightarrow Y
$$

definida por ev $(f, x)=f(x)$, para todo $x \in X$ e $f \in m(X, Y)$, é contínua.

A função ev acima é chamada função avaliação.

Teorema 1.1.2. Sejam $X$ um espaço de Hausdorff localmente compacto e $m(X, Y)$ com a topologia compacto-aberta. Para um espaço arbitrário $Z \in$ Top, uma função

$$
f: X \times Z \rightarrow Y
$$


é contínua se, e somente se, a função

$$
\tilde{f}: Z \rightarrow m(X, Y)
$$

definida por $\tilde{f}(z)(x)=f(x, z)$, para todo $(x, z) \in X \times Z$, é contínua.

Este último resultado é conhecido como a Lei exponencial e dizemos que as aplicações contínuas $f$ e $\tilde{f}$ são adjuntas segundo a Lei exponencial.

Agora, se $\left(X, x_{0}\right),\left(Y, y_{0}\right) \in T o p_{0}$, consideremos $m_{0}(X, Y)$ o espaço de todas as aplicações contínuas $f:\left(X, x_{0}\right) \rightarrow\left(Y, y_{0}\right)$ com a topologia compacto-aberta.

Um exemplo bastante conhecido de um espaço de funções baseadas é obtido considerando $\left(X, x_{0}\right)$ como a esfera unitária unidimensional $S^{1}$ de $\mathbb{R}^{2}$, com ponto base $e_{0}=(0,1)$. O espaço $m_{0}\left(S^{1}, Y\right)$ é normalmente denotado por $\Omega Y$ e é chamando o espaço dos laços associado a $\left(Y, y_{0}\right)$. Podemos ver $\Omega Y$ como o espaço de todas as aplicações contínuas $\alpha: I \rightarrow Y$ tal que $\alpha(0)=\alpha(1)=y_{0}$, onde $I$ é o intervalo unitário [0,1].

Um importante espaço associado aos espaços $\left(X, x_{0}\right)$ e $\left(Y, y_{0}\right)$ é o produto smash $X \wedge Y$ definido como segue: considere o espaço $X \vee Y=X \times\left\{y_{0}\right\} \cup\left\{x_{0}\right\} \times Y$, chamado produto wedge de $X$ e $Y$, considerado como um subespaço do produto $X \times Y$, então $X \wedge Y=$ $(X \times Y) /(X \vee Y)$. Denotamos os pontos de $X \wedge Y$ por $x \wedge y$, com $x \in X$ e $y \in Y$; em particular, $X \wedge Y$ é um espaço baseado com ponto base $x_{0} \wedge y_{0}$. Observemos que no caso $\left(X, x_{0}\right)=\left(S^{1}, e_{0}\right)$ e $\left(Y, y_{0}\right)$ arbitrário, o espaço $S^{1} \wedge Y=\Sigma Y$ é chamado suspensão de $\left(Y, y_{0}\right)$. Podemos utilizar também o intervalo $I$, ao invés de $S^{1}$, para definir $\Sigma Y$; de fato, considere $S^{1}$ como o conjunto de todos os números complexos $e^{2 \pi i t}$, parametrizado por $t \in I$, e estabelecemos um homeomorfismo

$$
\Sigma Y \cong(I \times Y) /\left(I \times\left\{y_{0}\right\} \cup \partial I \times Y\right)
$$

onde $\partial I=\{0,1\}$. Sempre que considerarmos $\Sigma Y$ desta forma, denotaremos seus elementos por $[t, y]$, entendendo que $[t, y]$ representa a classe de $(t, y)$ módulo $I \times\left\{y_{0}\right\} \cup \partial I \times Y$. O ponto base de $\Sigma Y$ é denotado por $*$.

O seguinte resultado pode ser encontrado em [37]

Teorema 1.1.3. Para todo $\left(X, x_{0}\right),\left(Y, y_{0}\right) \in$ Top $_{0}$, existe uma bijeção

$$
\Phi: m_{0}(\Sigma X, Y) \rightarrow m_{0}(X, \Omega Y)
$$

se $X$ é Hausfdorff, então $\Phi$ é continua.

Ainda por [37], para $X, Y, Z \in T o p$, temos:

(i) se $X$ é um espaço de Hausdorff localmente compacto e $Y, Z$ são espaços arbitrários, então $m(X \times Z, Y) \cong m(Z, m(X, Y))$;

(ii) se $X$ e $Y$ são espaços de Hausdorff compactos, então $m_{0}(X \wedge Y, Z) \cong m_{0}\left(X, m_{0}(Y, Z)\right)$; (iii) se $X, Y, Z$ são espaços baseados e $X$ é Hausdorff, então $m_{0}(X, Y \times Z) \cong m_{0}(X, Y) \times$ $m_{0}(X, Z)$.

Duas aplicações contínuas $f, g: X \rightarrow Y$ são homotópicas, notação $f \sim g$, se existe uma aplicação contínua $F: X \times I \rightarrow Y$, chamada homotopia, tal que $F(x, 0)=f(x)$ e 
$F(x, 1)=g(x)$, para todo $x \in X$. Homotopia de aplicações é uma relação de equivalência, e denotamos por Htop a categoria cujos objetos são espaços topológicos e morfismos são as classes de equivalência de aplicações homotópicas.

Particionemos agora o conjunto $m(X, Y)$ em classes de equivalência de homotopias chamadas classes de homotopias livres. O conjunto de todas essas classes será denotado por $[X, Y]$.

Para o caso de $m_{0}(X, Y)$ as classes serão definidas pela relação de homotopia baseada da seguinte forma: $f, g \in m_{0}(X, Y)$ são homotópicas baseadas se existe uma aplicação contínua

$$
H: X \times I \rightarrow Y
$$

tal que, para todo $x \in X, t \in I, H(x, 0)=f_{0}(X), H(x, 1)=f_{1}(x)$ e $H\left(x_{0}, t\right)=y_{0}$. Homotopia baseada é uma relação de equivalência e as classes de equivalência em $m_{0}(X, Y)$ serão chamadas de classes de homotopias baseadas. O conjunto dessas classes será denotado por $[X, Y]_{0}$.

Por [37] temos

Teorema 1.1.4. Para todo $\left(X, x_{0}\right),\left(Y, y_{0}\right) \in$ Top $_{0}$, a função

$$
\Phi: m_{0}(\Sigma X, Y) \rightarrow m_{0}(X, \Omega Y)
$$

definida no Teorema 1.1.3 induz uma bijeção

$$
\bar{\Phi}:[\Sigma X, Y]_{0} \rightarrow[X, \Omega Y]_{0}
$$

Ainda em [37], para todo $\left(X, x_{0}\right),\left(Y, y_{0}\right) \in T_{o p_{0}}$, podemos encontrar:

(i) $[X, \Omega Y]_{0}$ é um grupo;

(ii) $[\Sigma X, Y]_{0}$ é um grupo;

(iii) a bijeção $\bar{\Phi}$ é um isomorfismo de grupos.

Consideremos agora o espaço baseado $X$ como sendo a esfera unitária $n$-dimensional $S^{n}$, para $n \geq 0$, e adotemos a notação

$$
\left[S^{n}, Y\right]_{0}=\pi_{n}\left(Y, y_{0}\right)
$$

para todo $\left(Y, y_{0}\right) \in T_{o p_{0}}$ e todo $n \geq 0$.

Temos que para todo $\left(Y, y_{0}\right) \in T_{o p_{0}}$ e para todo $n \geq 1, \pi_{n}\left(Y, y_{0}\right)$ é um grupo e para todo $n \geq 2, \pi_{n}\left(Y, y_{0}\right)$ é um grupo comutativo.

$\mathrm{O}$ grupo $\pi_{n}\left(Y, y_{0}\right)$ é o n-ésimo grupo de homotopia de $\left(Y, y_{0}\right)$. O conjunto baseado $\left[S^{0}, Y\right]_{0}=\pi_{0}\left(Y, y_{0}\right)$ é apenas o conjunto de todas as componentes conexas por caminhos de $Y$. Em alguns casos $\pi_{0}\left(Y, y_{0}\right)$ tem uma estrutura de grupo, mas não o consideraremos como um grupo de homotopia. Observe que os grupos de homotopia de espaços contráteis e de espaços discretos são todos triviais. 


\subsection{Fibrações e co-fibrações}

Daqui por diante, quando falarmos em aplicações, já estaremos considerando que essas são contínuas. Consideremos agora a notação $(E, p, B)$ para $E, B \in T o p$ e $p: E \rightarrow B$ uma aplicação. Os espaços $E$ e $B$ são chamados de fonte e meta de $p$, respectivamente.

Dados $(Y, g, B)$ e $(A, f, B)$, um pull back de $g$ e $f$ em Top é definido por $(X, \bar{f}, Y)$ e $(X, \bar{g}, A)$ tais que $g \bar{f}=f \bar{g}$ e satisfazendo:

propriedade universal: dados $(Z, h, Y)$ e $(Z, k, A)$ tais que $g h=f k$, existe uma única $\ell: Z \rightarrow X$ tal que $\bar{f} \ell=h$ e $\bar{g} \ell=k$.

Essa situação é descrita pelo seguinte diagrama comutativo:

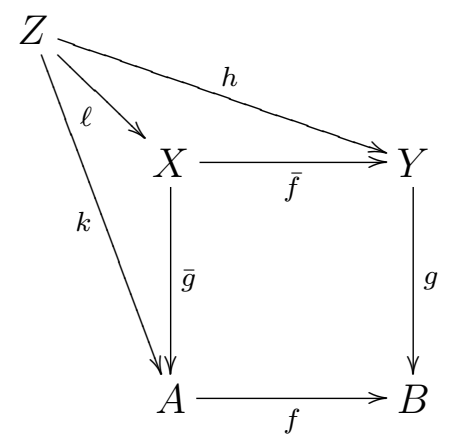

Assumindo por um momento que os espaços pull back existam em Top, então a propriedade universal nos mostra que, para $(Y, g, B)$ e $(A, f, B)$, o espaço $X$ desse modo criado é único a menos de homeomorfismo. O espaço $X=A_{g} \sqcap_{f} Y=\{(a, y) \in A \times Y \mid f(a)=g(y)\}$ é chamado o espaço pull back.

Olhando para a noção dual de pull back, dados $(A, f, B)$ e $(A, g, Y)$, um push out de $f$ e $g$ em Top, é um par, digamos $(Y, \bar{f}, X)$ e $(B, \bar{g}, X)$ tal que $\bar{f} g=\bar{g} f$ e satisfazendo: propriedade universal: dados $(B, k, Z)$ e $(Y, h, Z)$ tais que $k f=h g$, existe uma única $\ell: X \rightarrow Z$ tal que $\ell \bar{f}=h$ e $\ell \bar{g}=k$.

Ilustramos a situação no seguinte diagrama comutativo:

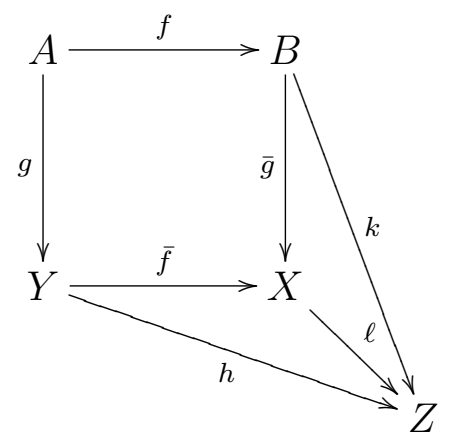

A categoria de espaços topológicos e aplicações, possuem push outs e o espaço push out de $f$ e $g$ é $X=B_{g} \sqcup_{f} Y$ (ou qualquer espaço homeomorfo a ele), o qual é o conjunto de todas as classes de equivalência da soma topológica $B \sqcup Y$ segundo a relação de equivalência gerada por

$$
b \mathcal{R} y \Leftrightarrow \exists a \in A \mid b=f(a), y=g(a) .
$$


Temos duas leis de composição interessantes:

- lei de composição horizontal: dados $A \subset Y$ fechado, $(A, f, B)$ e $(B, g, C)$, temos

$$
C \sqcup_{g}\left(B \sqcup_{f} Y\right) \cong C \sqcup_{g f} Y
$$

- lei de composição vertical: dados $A \subset Y$ fechado, $Y \subset Y^{\prime}$ fechado e $(A, f, B)$, temos

$$
\left(B \sqcup_{f} Y\right) \sqcup_{\bar{f}} Y^{\prime} \cong B \sqcup_{f} Y^{\prime}
$$

Agora, para todo $B \in$ Top, seja $B^{I}=m(I, B)$ o espaço de todos os caminhos em $B$. Considere a aplicação avaliação $e v_{0}: B^{I} \rightarrow B$ obtida avaliando cada caminho em $B$ no ponto $0 \in[0,1]$. Dado $(E, p, B)$ e considerando $\left(B^{I}, e v_{0}, B\right)$ formamos o espaço pull back

$$
B^{I} \sqcap E=B_{p}^{I} \sqcap_{e v_{0}} E .
$$

Considere as aplicações $p^{I}: E^{I} \rightarrow B^{I}$ e $e \hat{v}_{0}: E^{I} \rightarrow E$ definidas por $p^{I}(\lambda)=p \lambda$ e $e \hat{v}_{0}(\lambda)=\lambda(0)$, para todo $\lambda \in E^{I}$. Note que $p e \hat{v}_{0}=e v_{0} p^{I}$. Então a propriedade universal do pull back nos leva a uma única aplicação $\pi: E^{I} \rightarrow B^{I} \sqcap E$ tal que $\bar{p} \pi=p^{I}$ e $e \bar{v}_{0} \pi=e \hat{v}_{0}$ e temos o seguinte diagrama comutativo:

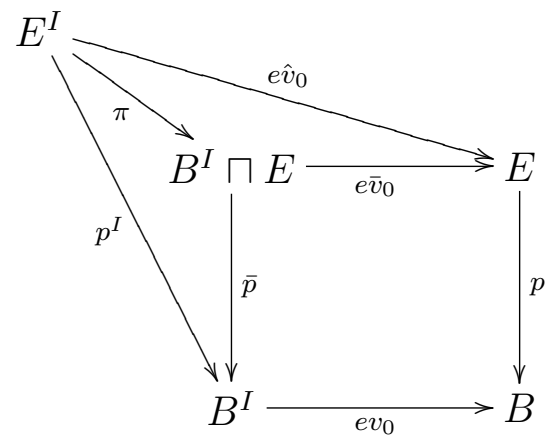

Se existe uma aplicação $\Gamma: B^{I} \sqcap E \rightarrow E^{I}$ tal que $\pi \Gamma=1_{B^{I} \sqcap E}$, dizemos que $\Gamma$ é uma função levantamento de $(E, p, B)$.

O seguinte resultado pode ser encontrado em [37]:

Teorema 1.2.1. Dado $(E, p, B)$ com $E, B \in$ Top e p uma aplicação, temos as seguintes equivalências:

(1) Para todo $(A, g, E)$ e toda homotopia $H: A \times I \rightarrow B$ tal que $H(-, 0)=p g$, existe uma homotopia $G: A \times I \rightarrow E$ tal que $G(-, 0)=g$ e $p G=H$;

(2) para todo $(D, q, A)$, todo par de aplicações $(g, h)$ para o qual temos o diagrama comutativo:

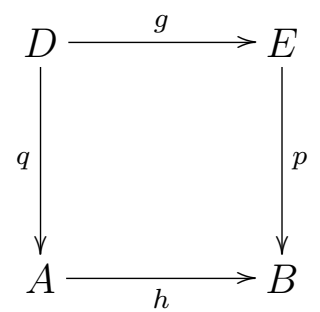


e toda homotopia $H: A \times I \rightarrow B$ de h existe uma homotopia $G: D \times I \rightarrow E$ de g tal que $p G=H\left(q \times 1_{I}\right)$;

(3) existe uma função levantamento $\Gamma$ para $(E, p, B)$.

Se $(E, p, B)$ satisfaz as condições equivalentes do teorema acima, dizemos que é uma fibração. Sendo (E, p, B) uma fibração, dado $b \in B$, o subsepaço $p^{-1}(b)=E_{b}$ de $E$ é a fibra de $p$ sobre $b$.

Temos também em [37] os seguintes resultados:

Teorema 1.2.2. Seja $(E, p, B)$ uma fibração com B conexo por caminhos. Então todas as fibras de $p$ têm o mesmo tipo de homotopia e p é sobrejetiva.

Teorema 1.2.3. Toda aplicação $f: X \rightarrow Y$ pode ser fatorada como uma equivalência de homotopia seguida por uma fibração.

Consideremos agora um espaço $X \in$ Top e um subespaço $A \subset X$, dizemos que $A$ é um retrato de deformação forte de $X$ se existe uma homotopia $H: X \times I \rightarrow X$ tal que

$$
\begin{gathered}
H(x, 0)=x, x \in X \\
H(x, 1) \in A, x \in X \\
H(a, t)=a,(a, t) \in A \times I .
\end{gathered}
$$

A homotopia $H$ é um retrato de deformação forte de $X$ sobre $A$. A aplicação $r=H(-, 1): X \rightarrow A$ é uma retração e $A$ é um retrato de $X$. Assim, um retrato $A$ de $X$ com retração $r: X \rightarrow A$ é um retrato de deformação forte de $X$ se

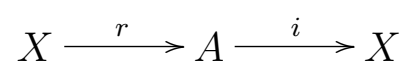

é $A$-homotópico relativo a $1_{X}$.

Por [37] temos:

Teorema 1.2.4. Seja A um subespaço fechado de $X$. As seguintes afirmações são equivalentes:

(1) para quaisquer duas aplicações $f: X \times\{0\} \rightarrow Z$ e $G: A \times I \rightarrow Z$ a qual coincide quando restrita a $A \times\{0\}$ existe uma aplicação $F: X \times I \rightarrow Z$ tal que F restrita a $X \times\{0\}$ é $f$ e F restrita a $A \times I$ é $G$;

(2) o espaço $\hat{X}=X \times\{0\} \cup A \times I$ é um retrato de $X \times I$;

(3) $\hat{X}$ é um retrato de deformação forte de $X \times I$.

Dizemos que $(A, i, X)$, com $A \subset X$ fechado e $i$ a aplicação inclusão, é uma co-fibração se satisfaz qualquer uma das condições equivalentes do teorema anterior.

Um exemplo de co-fibração bastante conhecido é $(Y, i, C Y)$, onde $\left(Y, y_{0}\right) \in T_{o p}$ e $C Y=(I \times Y) /\left(I \times\left\{y_{0}\right\} \cup\{0\} \times Y\right)$ é o cone de $\left(Y, y_{0}\right)$. O cone $C Y$ está intimamente relacionado a suspensão de $Y$ : de fato, $\Sigma Y=C Y / Y$. 
A co-fibração $(Y, i, C Y)$ dá origem a uma outra importante cofibração, dessa vez relacionada a uma aplicação baseada $f:\left(Y, y_{0}\right) \rightarrow\left(Z, z_{0}\right)$; estamos nos referindo a co-fibração mapping cone da aplicação baseada $f$ :

$$
\left(Z, \bar{i}, Z_{i} \sqcup_{f} C Y\right) .
$$

O espaço $Z_{i} \sqcup_{f} C Y$ é conhecido como mapping cone da aplicação $f$ e é denotado por $C_{f}$.

Teorema 1.2.5. Toda aplicação $f: A \rightarrow B$ pode ser fatorada como uma co-fibração seguida por uma equivalência de homotopia.

Temos, também, por [23] pág. 184:

Proposição 1.2.6. Seja $u: A \rightarrow X$ uma co-fibração fechada, onde $X$ é um espaço regular localmente compacto. Então

$$
u^{*}: m(X, E) \rightarrow m(A, E)
$$

é uma fibração para todos os espaços E.

\subsection{Seqüências exatas de homotopia}

Comecemos relembrando o caso co-variante. Para uma aplicação $f:\left(Y, y_{0}\right) \rightarrow\left(Z, z_{0}\right)$ temos que $\left(P Z, e v_{1}, Z\right)$ é uma fibração, onde $P Z=m_{0}(I, B)$ é o espaço dos caminhos baseados e $e v_{1}$ é a aplicação avaliação no valor 1 . Sua fibra sobre $z_{0}$ é o espaço dos laços $\Omega Z$ (laço baseados em $z_{0}$ ). Consideremos agora o espaço pull back $L_{f}=Y \sqcap P Z$ de $e v_{1}$ e $f ;\left(L_{f}, e \bar{v}_{1}, Y\right)$ é também uma fibração e sua fibra sobre $y_{0}$ é $\Omega Z$. Desse modo obtemos uma seqüência de espaços topológicos e aplicações

$$
\Omega Z \underset{j}{\longrightarrow} L_{f} \underset{e \bar{v}_{1}}{\longrightarrow} Y \underset{f}{\longrightarrow} Z
$$

na qual $j$ é a inclusão de $\Omega Z$ em $L_{f}$. Na verdade, por iterações dessas aplicações obtemos uma sequência de espaços e aplicações a qual se estende indefinidamente à esquerda:

$$
\begin{gathered}
\cdots \underset{\Omega^{n+1} f}{\longrightarrow} \Omega^{n+1} Z \underset{\Omega^{n} j}{\longrightarrow} \Omega^{n} L_{f} \underset{\Omega^{n} e \bar{v}_{1}}{\longrightarrow} \Omega^{n} Y \underset{\Omega^{n} f}{\longrightarrow} \\
\Omega^{n} Z \underset{\Omega^{n-1} j}{\longrightarrow} \cdots \underset{j}{\longrightarrow} L_{f} \underset{e \bar{v}_{1}}{\longrightarrow} Y \underset{f}{\longrightarrow} Z .
\end{gathered}
$$

Esta é a seqüência exata longa de espaços e aplicações associada à $f$.

Por [37] temos os seguintes resultados: 
Teorema 1.3.1. Para todo $\left(X, x_{0}\right),\left(Y, y_{0}\right),\left(Z, z_{0}\right) \in$ Top $_{0}$ e toda aplicação $f:\left(Y, y_{0}\right) \rightarrow$ $\left(Z, z_{0}\right)$, a seqüência de conjuntos baseados e grupos

$$
\begin{gathered}
\cdots \underset{\Omega^{n+1} f}{\longrightarrow}\left[X, \Omega^{n+1} Z\right]_{0} \underset{\Omega^{n} j_{*}}{\longrightarrow}\left[X, \Omega^{n} L_{f}\right]_{0} \underset{\Omega^{n} e \bar{v}_{1 *}}{\longrightarrow}\left[X, \Omega^{n} Y\right]_{0} \underset{\Omega^{n} f_{*}}{\longrightarrow} \cdots \\
\cdots \underset{j_{*}}{\longrightarrow}\left[X, L_{f}\right]_{0} \underset{e \bar{v}_{1 *}}{\longrightarrow}[X, Y]_{0} \underset{f_{*}}{\longrightarrow}[X, Z]_{0}
\end{gathered}
$$

induzida pela seqüência exata longa de espaços e aplicações associada à $f$, é exata.

Lema 1.3.2. Seja $(E, p, B)$ uma fibração com fibra $F$ sobre $b_{0} \in B$. Então os espaços $L_{p}$ e F têm o mesmo tipo de homotopia.

Teorema 1.3.3. Sejam $(E, p, B)$ uma fibração com fibra $F$ sobre $b_{0} \in B$ e $e_{0} \in F$ visto como ponto-base para $F$ e E. Então, para todo $\left(X, x_{0}\right) \in T_{o p_{0}}$, a seguinte seqüência de conjuntos baseados e grupos é exata:

$$
\begin{gathered}
\cdots \underset{\Omega^{n+1} p_{*}}{\longrightarrow}\left[X, \Omega^{n+1} B\right]_{0} \underset{\Omega^{n} j_{*}}{\longrightarrow}\left[X, \Omega^{n} F\right]_{0} \underset{\Omega^{n} i_{*}}{\longrightarrow}\left[X, \Omega^{n} E\right]_{0} \underset{\Omega^{n} p_{*}}{\longrightarrow} \cdots \\
\cdots[X, F]_{0} \underset{i_{*}}{\longrightarrow}[X, E]_{0} \underset{p_{*}}{\longrightarrow}[X, B]_{0} .
\end{gathered}
$$

Agora especificamos $\left(X, x_{0}\right)$ como sendo a 0 -esfera unitária baseada $\left(S^{0}, \mathrm{e}_{0}\right)$. Recordemos que $\pi_{0}\left(Y, y_{0}\right)$ é o conjunto de todas as componentes conexas por caminhos de $Y$. Então pelo teorema anterior temos o seguinte:

Teorema 1.3.4. Sejam $(E, p, B)$ uma fibração com fibra $F=p^{-1}\left(b_{0}\right)$ e $e_{0} \in F$ o ponto base para $F$ e E. Então, a seguinte seqüência de conjuntos baseados e grupos

$$
\begin{gathered}
\cdots \underset{p_{*}(n+1)}{\longrightarrow} \pi_{n+1}\left(B, b_{0}\right) \underset{j_{*}(n)}{\longrightarrow} \pi_{n}\left(F, e_{0}\right) \underset{i_{*}(n)}{\longrightarrow} \pi_{n}\left(E, e_{0}\right) \underset{p_{*}(n)}{\longrightarrow} \cdots \\
\cdots \underset{j_{*}(0)}{\longrightarrow} \pi_{0}\left(F, e_{0}\right) \underset{i_{*}(0)}{\longrightarrow} \pi_{0}\left(E, e_{0}\right) \underset{p_{*}(0)}{\longrightarrow} \pi_{0}\left(B, b_{0}\right) .
\end{gathered}
$$

é exata.

Para o caso contravariante, seja $f:\left(Y, y_{0}\right) \rightarrow\left(Z, z_{0}\right)$ uma aplicação que preserva ponto base e seja $\left(Z, \bar{i}, C_{f}\right)$ sua co-fibração mapping cone. Agora considere a aplicação constante $c_{z_{0}}: Z \rightarrow\left\{z_{0}\right\}$ e, usando a lei de composição horizontal e a relação entre $\Sigma Y$ e $C Y$, temos:

$$
\left\{z_{0}\right\} \sqcup_{c_{z_{0}}} C_{f} \cong\left\{z_{0}\right\} \sqcup_{c_{z_{0}} f} C Y \cong \Sigma Y
$$

Então construímos uma seqüência infinita de espaços e aplicações por sucessivas iterações:

$$
Y \underset{f}{\longrightarrow} Z \underset{\bar{i}}{\longrightarrow} C_{f} \underset{c_{z_{0}}^{-}}{\longrightarrow} \Sigma Y \underset{\Sigma f}{\longrightarrow} \Sigma Z \underset{\Sigma \bar{i}}{\longrightarrow} \cdots
$$


Fixado $\left(X, x_{0}\right) \in T o p_{0}$, a aplicação baseada $f$ induz uma função que preserva pontobase

$$
f^{*}:[Z, X]_{0} \rightarrow[Y, X]_{0}
$$

e um homomorfismo de grupos

$$
\Sigma f^{*}:[\Sigma Z, X]_{0} \rightarrow[\Sigma Y, X]_{0}
$$

Teorema 1.3.5. Para todo $\left(X, x_{0}\right),\left(Y, y_{0}\right),\left(Z, z_{0}\right) \in$ Top $_{0}$ e toda aplicação $f:\left(Y, y_{0}\right) \rightarrow$ $\left(Z, z_{0}\right)$, a seqüência de conjuntos baseados e grupos

$$
\begin{aligned}
& \cdots \underset{\Sigma^{n+1} f^{*}}{\longrightarrow}\left[\Sigma^{n+1} Y, X\right]_{0} \underset{\Sigma^{n} c_{z_{0}}^{-}{ }^{*}}{\longrightarrow}\left[\Sigma^{n} C_{f}, X\right]_{0} \underset{\Sigma^{n} \bar{i}^{*}}{\longrightarrow}\left[\Sigma^{n} Z, X\right]_{0} \underset{\Sigma^{n} f^{*}}{\longrightarrow} \cdots \\
& \cdots \underset{c_{z_{0}}{ }^{*}}{\longrightarrow}\left[C_{f}, X\right]_{0} \underset{\bar{i}^{*}}{\longrightarrow}[Z, X]_{0} \underset{f^{*}}{\longrightarrow}[Y, X]_{0}
\end{aligned}
$$

é exata.

Lema 1.3.6. Seja $(Y, j, Z)$ uma co-fibração, então os espaços $C_{j}$ e $Z / Y$ têm o mesmo tipo de homotopia.

Deste último lema, e para manter uma coerência entre os resultados nos conceitos duais de fibração e co-fibração, dado $(Y, i, Z)$ uma cofibração, o espaço quociente $Z / Y$ será chamado de co-fibra de $(Y, i, Z)$. Temos também o seguinte resultado:

Teorema 1.3.7. Sejam $\left(X, x_{0}\right) \in$ Top $_{0}$ e $(Y, j, Z)$ uma co-fibração. Para todo ponto base $y_{0} \in Y \subset Z$, a seqüência de conjuntos baseados e grupos

$$
\begin{gathered}
\cdots \underset{\Sigma^{n+1} j^{*}}{\longrightarrow}\left[\Sigma^{n+1} Y, X\right]_{0} \underset{\Sigma^{n} c_{y_{0}}{ }^{*}}{\longrightarrow}\left[\Sigma^{n} Z / Y, X\right]_{0} \underset{\Sigma^{n} \bar{i}^{*}}{\longrightarrow}\left[\Sigma^{n} Z, X\right]_{0} \underset{\Sigma^{n} j^{*}}{\longrightarrow} \cdots \\
\cdots \underset{c_{y_{0}}{ }^{*}}{\longrightarrow}[Z / Y, X]_{0} \underset{\bar{i}^{*}}{\longrightarrow}[Z, X]_{0} \underset{j^{*}}{\longrightarrow}[Y, X]_{0}
\end{gathered}
$$

é exata.

Chamamos a seqüência do Teorema 1.3 .7 de seqüência exata da co-fibração $(Y, j, Z)$.

\subsection{Alguns lemas}

Todos os espaços considerados no que segue serão conexos por caminhos e com ponto-base se o contrário não for especificado.

Recordemos que a fibração mapping track ou fibração mapping path associada a uma aplicação dada $f: X \rightarrow Y$, é a fibração $p_{f}: P_{f}=X \sqcap_{\epsilon_{0}} Y^{I} \rightarrow Y$ definida como a diagonal de um diagrama pull back 


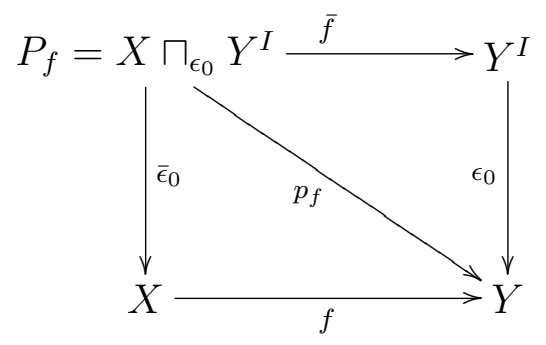

por $p_{f}(x, \lambda)=\lambda(1)$ [37] pág. 45, no qual o espaço $P_{f}=X \sqcap_{\epsilon_{0}} Y^{I}$ é chamado mapping track de $f$ ou espaço mapping path [10] 6.17.

Lema 1.4.1. A aplicação $\overline{e v}_{0}$ é uma equivalência de homotopia de $X$ e $P_{f}$ com inversa $s: X \rightarrow P_{f}, s: x \mapsto\left(x, c_{f(x)}\right)$ sobre $Y$, isto é, tal que $p_{f} s=f$.

Demonstração: [37] 2.2.7, [10] 6.18.

A fibra de $p_{f}, F_{f}=p_{f}^{-1}\left(y_{0}\right)$ é chamada a fibra de homotopia de $f$. Uma caracterização desse espaço será dada no Lema 1.4.3 mais a frente.

Lema 1.4.2. Se $f$ é uma fibração com fibra $F$, a equivalência de homotopia s é uma equivalência de fibra de homotopia, isto é, $F \sim F_{f}$.

Demonstração: [10] 6.18.

A prova do próximo lema pode ser feita de um modo alternativo, como em [10] 6.39, 6.40, usando diretamente a construção do mapping track. Seguimos aqui o caminho escolhido por [37].

Seja $f:\left(X, x_{0}\right) \rightarrow\left(Y, y_{0}\right)$ uma aplicação baseada. Considere $f$ uma fibração com fibra $F=f^{-1}\left(y_{0}\right)$ sobre o ponto base $y_{0}$ e inclusão $i: F \rightarrow X$. Seja $P Y$ o espaço dos caminhos baseados $\left\{\lambda \in Y^{I}: \lambda(0)=y_{0}\right\}$. O espaço pull back $L_{f}=X \sqcap P Y$ definido pelo diagrama

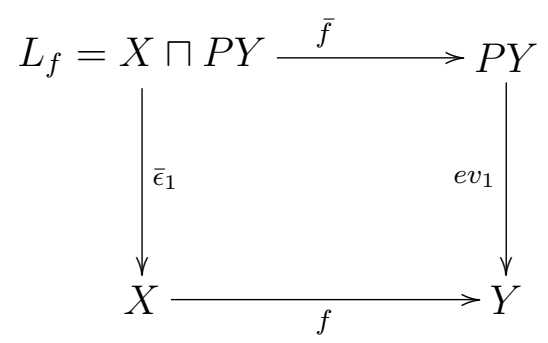

será chamado espaço baseado mapping track de $f$ e é o espaço total da fibração $\overline{e v}_{1}: L_{f} \rightarrow$ $X$, cuja fibra sobre $x_{0}$ pode ser facilmente reconhecida como o espaço dos laços baseados em $y_{0}$, isto é, $\bar{e} \bar{v}_{1}^{-1}\left(x_{0}\right)=\Omega Y$ (isso é verdade apenas se a aplicação $f$ for baseada!) [37] 2.2.2. Também, como $f$ é uma fibração, por [37] 3.1.3, a aplicação $h: F \rightarrow L_{f}$, $h: x \mapsto\left(x, c_{y_{0}}\right)$ é uma equivalência de homotopia e $F \sim L_{f}$.

Lema 1.4.3. A fibra do mapping track é o espaço baseado mapping track (a menos de homotopia). 


\section{Demonstração:}

$$
\begin{gathered}
p_{f}^{-1}\left(y_{0}\right)=\left\{(x, \lambda) \in X \sqcap_{e v_{0}} Y^{I}: \lambda(1)=y_{0}\right\}= \\
=\left\{(x, \lambda) \in X \times Y^{I}: \lambda(0)=y_{0}, \lambda(1)=f(x)\right\}=X \sqcap P Y=L_{f} .
\end{gathered}
$$

E mais, consideremos novamente a construção do espaço baseado mapping track para a aplicação $\overline{e v}$. Obtemos o espaço baseado mapping track $L_{\bar{e}_{1}}$ com a aplicação $\bar{e}_{1}$ definida pelo diagrama pull back

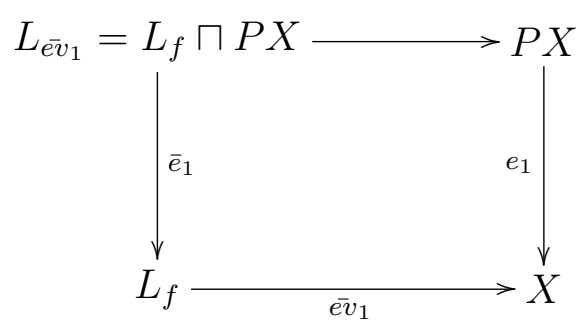

Por [37] 2.2.2, $\bar{e}_{1}$ é uma fibração e procedendo como acima, sua fibra sobre o ponto base $\bar{e}_{1}^{-1}\left(c_{x_{0}}\right)=\Omega X$ (onde os laços em $X$ são baseados em $x_{0}$ ). Também, como $\bar{e} \bar{v}_{1}$ é na verdade uma fibração com fibra $\Omega Y$, a aplicação $g: \Omega Y \rightarrow L_{\bar{e}_{1}}, g: \lambda \mapsto\left(\left(x_{0}, \lambda\right), c_{x_{0}}\right)$, é uma equivalência de homotopia, isto é, $L_{e_{\bar{v}}} \sim \Omega Y$ por [37] 3.13 .

Podemos resumir essa construção no seguinte diagrama

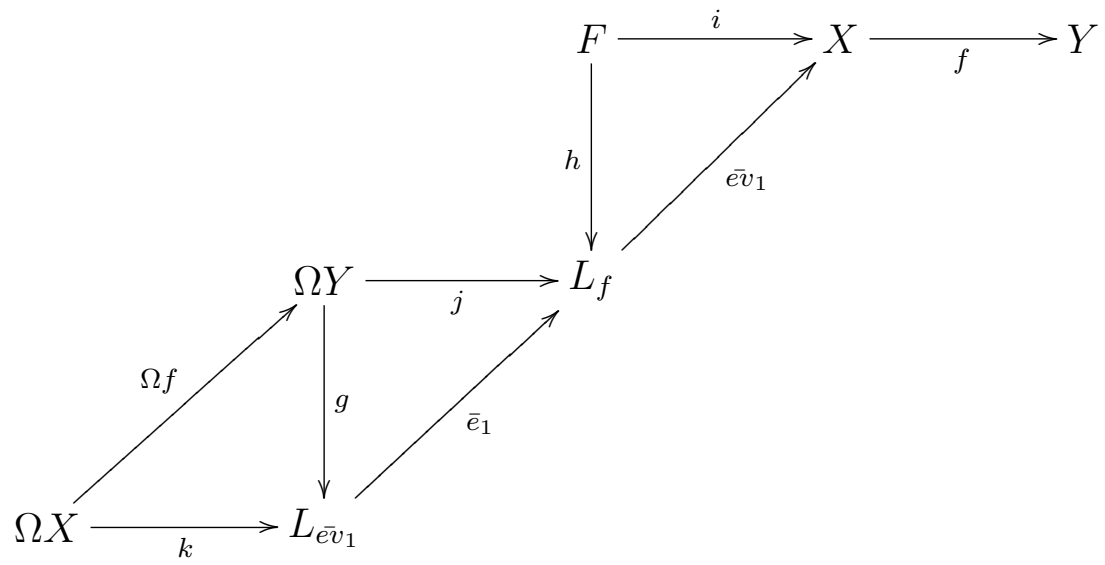

no qual $j: \Omega Y \rightarrow L_{f}, j: \beta \mapsto\left(x_{0}, \beta\right)$, e $k: \Omega X \rightarrow L_{\bar{e}_{1}}, k: \alpha \mapsto\left(\left(x_{0}, c_{y_{0}}\right), \alpha\right)$ são as inclusões de fibras, e temos que os dois triângulos da direita comutam, $\bar{e}_{1} g=j$ e $\bar{\epsilon}_{1} h=i$. Ainda, podemos provar que o triângulo da esquerda é também comutativo a menos de homotopia, ou seja, $g \Omega f \sim k$. Fazemos isso usando a seguinte homotopia

$$
\begin{gathered}
H: \Omega X \times I \rightarrow L_{\bar{\epsilon}_{1}}, \\
H:(\alpha, s) \mapsto\left(\left(x_{0}, f \alpha(s t)\right), \alpha(1+s t-t)\right) .
\end{gathered}
$$


De fato, a imagem do ponto base está no caminho $\gamma_{s}(t)=\left(\left(x_{0}, f \alpha(s t)\right), \alpha(1+t s-t)\right)$ que sempre pertence a $L_{\bar{\epsilon}_{1}}$ já que, no ponto inicial $t=0, \gamma_{s}(0)=\left(\left(x_{0}, c_{y_{0}}\right), c_{x_{0}}\right)$, no ponto final $t=1, \gamma_{s}(1)=\left(\left(x_{0}, f \alpha(s)\right), \alpha(s)\right)$. Também,

$$
\begin{gathered}
H(\alpha, 1)(t)=\left(\left(x_{0}, f \alpha(t)\right), c_{x_{0}}\right)=g \Omega f \\
H(\alpha, 0)(t)=\left(\left(x_{0}, c_{y_{0}}\right), \alpha^{-1}(t)\right)=k \beta
\end{gathered}
$$

sendo $\beta: \Omega X \rightarrow \Omega X$ o laço inverso $\beta: \alpha \mapsto \alpha^{-1}$ e é uma auto-equivalência de homotopia. Assim, $g \Omega f \sim_{H} k \beta \sim k$.

Provamos o seguinte lema [10] 6.40, [37] 3.1.

Lema 1.4.4. Seja $f: X \rightarrow Y$ uma fibração com fibra $F$. Então quaisquer duas aplicações consecutivas na seqüência

$$
\cdots \longrightarrow \Omega F \longrightarrow \Omega X \underset{\Omega f}{\longrightarrow} \Omega Y \longrightarrow F \underset{i}{\longrightarrow} X \underset{f}{\longrightarrow} Y
$$

têm o tipo de homotopia de uma fibração precedida pela inclusão de sua fibra.

Veja [47] pág. 166 para as definições de simplicidade e prova do seguinte lema:

Lema 1.4.5. Seja Y um espaço simples. Então, cada auto-equivalência de homotopia s de $Y$ pode ser considerada baseada (como uma aplicação, já que $Y$ é conexo por caminhos) e baseada como homotopia, ou seja, as homotopias $s s^{-1} \sim 1 \sim s^{-1} s$ preservam ponto-base.

Lema 1.4.6. Seja $f: X \rightarrow Y$ uma aplicação e $\sigma: Y \rightarrow Y$ uma auto-equivalência de homotopia baseada com inversa $\tau$. Então, as fibras de homotopia $F_{f}$ e $F_{\sigma f}$ são equivalentemente homotópicas.

Demonstração: Pela construção $P_{f} \sim P_{\sigma f}$, já que são ambos homotópicos a $X$. Mais precisamente, uma equivalência de homotopia é $s^{\prime} \bar{\epsilon}_{0}$, com inversa $s \bar{\epsilon}_{0}^{\prime}$, veja Lema 1.4.1. Mas isso pode não preservar fibra em geral, pois $f$ não é uma fibração, veja Lema 1.4.2. Explicitemos a prova. Recordemos que $F_{f}=L_{f}$, Lema 1.4.3. Considere as aplicações

$$
\begin{gathered}
\sigma^{\prime}: L_{f} \rightarrow L_{\sigma f}, \\
\sigma^{\prime}:(x, \lambda) \mapsto(x, \sigma \lambda),
\end{gathered}
$$

que é bem definida, já que $\sigma f(x)=\sigma \lambda(1)$, e

$$
\begin{gathered}
\tau^{\prime}: L_{\sigma f} \rightarrow L_{\tau \sigma f}, \\
\tau^{\prime}:(x, \lambda) \mapsto(x, \tau \lambda),
\end{gathered}
$$

que, analogamente, é bem definida. E a aplicação

$$
\begin{gathered}
h: L_{\tau \sigma f} \rightarrow L_{f}, \\
h:(x, \lambda) \mapsto\left(x, \lambda+\mu_{f(x)}\right),
\end{gathered}
$$


em que

$$
\mu_{f(x)}(t)=H(f(x), t),
$$

com $H: Y \times I \rightarrow Y$ sendo a homotopia baseada tal que $H(y, 0)=\tau \sigma(y), H(y, 1)=y$, $H\left(y_{0}, t\right)=y_{0}$.

Observe que $\mu_{f(x)}(0)=\tau \sigma f(x)=\lambda(1)$, e assim o caminho pode ser adicionado. Também, $\lambda(0)=y_{0}$ e $\mu_{f(x)}(1)=f(x)$, e assim $h$ está bem definida. Mostramos que $h \tau^{\prime}$ é uma inversa à esquerda de $\sigma^{\prime}$, isto é, $h \tau^{\prime} \sigma^{\prime} \sim 1_{L_{f}}$, pela homotopia

$$
\begin{gathered}
G: L_{f} \times I \rightarrow L_{f}, \\
G((x, \lambda), s)(t)= \begin{cases}\left(x, H\left(\lambda\left(\frac{2 s}{1+t}\right), t\right)\right), & 0 \leq s \leq \frac{1+t}{2}, \\
\left(x, \mu_{f(x)}\left(\frac{2 s}{1+t}-1+\frac{2 t s}{1+t}\right)\right), & \frac{1+t}{2} \leq s \leq 1 .\end{cases}
\end{gathered}
$$

Podemos verificar que $G$ está bem definida e obter a equivalência de homotopia necessária. 


\section{Capítulo}

\section{Fibrados e grupos de gauge}

O principal objetivo deste capítulo é apresentar a definição de um fibrado e de seu grupo de gauge e mostrar as principais propriedades desses objetos. Para esse propósito, introduziremos primeiro alguns fatos gerais sobre propriedades algébricas e topológicas de grupos contínuos e suas ações sobre espaços. Mais detalhes sobre as categorias que aparecem no texto, ver Apêndice no final do capítulo.

\subsection{Grupos topológicos e ações}

Definição 2.1.1. Dizemos que um espaço topológico $G$ é um grupo topológico com multiplicação $\tau: G \times G \rightarrow G$ se $\tau$ satisfaz as propriedades da lei multiplicativa de um grupo abstrato e a função $(g, h) \mapsto \tau\left(g, h^{-1}\right)$ é contínua.

Explicitamente, isso significa que $G$ é um grupo abstrato com multiplicação

$$
\begin{gathered}
\tau: G \times G \rightarrow G \\
\tau:(g, h) \rightarrow g h:=\tau(g, h),
\end{gathered}
$$

satisfazendo as propriedades de grupo

- associativa: $g(h k)=(g h) k$,

- unidade: $\exists u \in G \mid g u=u g=g$,

- inverso: $\forall g \in G, \exists g^{-1} \in G \mid g^{-1} g=g g^{-1}=u$,

que $G$ é um espaço topológico (na categoria oportuna), e mais, a função $\tau$ é uma função contínua, e também a função inversa que envia $g$ em $g^{-1}$.

Definição 2.1.2. Um grupo topológico $G$ age à direita de um espaço $X$ se existe uma aplicação, chamada ação à direita, $\phi: X \times G \rightarrow X$ tal que $\phi\left(\phi \times 1_{G}\right)=\phi\left(1_{X} \times \tau\right)$ e, para todo $x \in X, \phi(x, u)=x$.

Explicitamente

$$
\begin{gathered}
\phi: X \times G \rightarrow X, \\
\phi:(x, g) \mapsto x g=\phi(x, g),
\end{gathered}
$$


$\mathrm{e}$

$$
\phi(x, g h)=\phi(\phi(x, g), h), \phi(x, u)=x .
$$

Ações à esquerda são definidas simetricamente. Em particular, note que $(x, g) \mapsto x g$ é uma ação à direita e $(g, x) \mapsto x g^{-1}$ é uma ação à esquerda.

Sob essas condições, o par $(X, \phi)$ é chamado um $G$-espaço à direita/esquerda (também grupo de tranformação).

A translação à direita por $g, R_{g}: X \rightarrow X, x \mapsto x g$, é um homeomorfismo de $X$ com inversa $R_{g^{-1}}$. A aplicação $g \mapsto R_{g}$ é um homomorfismo de $G$ em Homeo $(X)$, o grupo dos homeomorfismos de $X$.

Definição 2.1.3. A ação $\phi$ de um grupo $G$ sobre um espaço $X$ é chamada efetiva se o núcleo de $g \mapsto L_{g}$ é trivial, e livre se $x g=x$ implica $g=u$.

Exemplo 2.1.4. Seja $H$ um subgrupo de $G$. O grupo multiplicação $G \times H \rightarrow G,(g, h) \mapsto$ gh é uma ação livre à direita de $H$ sobre $G$. Existe uma ação à esquerda similar. Também a ação adjunta $a d: G \times G \rightarrow G,(g, h) \mapsto h^{-1} g h$ é uma importante ação à direita de $G$ sobre o próprio $G$. O espaço é usualmente denotado por $A d(G)$.

A ação de $G$ sobre um $G$-espaço $X$ determina uma relação de equivalência $R$ sobre $X: x R x^{\prime}$ se, e somente se, existe um $g \in G$ tal que $x^{\prime}=x g$, o qual é consistente com a ação no sentido que, para todo $g \in G, x R x^{\prime}$ implica $(x g) R\left(x^{\prime} g\right)$. Observemos que $k(T o p)$ é a categoria dos espaços cujos subconjuntos compactamente fechados são fechados, chamados $k$-espaços (ver mais detalhes no Apêndice) e recorde que, dada uma relação de equivalência $R$ sobre $X \in k(T o p)$, já que $X / R$ tem a topologia final induzida pela aplicação quociente $p: X \rightarrow X / R$, então $X / R \in k(T o p)$ ([13] pág. 242). Isso não é totalmente verdade em $w H k(T o p)$, categoria dos $k$-espaços Hausdorff fraco, como vemos no seguinte resultado:

Lema 2.1.5. Seja $f: X \rightarrow Y$ uma proclusão em $k(T o p)$. Se $X$ é Hausdorff fraco, então $Y$ é Hausdorff fraco se, e somente se, $(f \times f)^{-1} \Delta_{Y}$ é fechado em $X \times X$.

A classe de equivalência de $x \in X$ é chamada a órbita $x G$ por $x$.

Definição 2.1.6. O k-espaço quociente $X / R$, também denotado por $X / G$, é chamado espaço das órbitas. A aplicação quociente é chamada projeção canônica e denotada por $p_{X}: X \rightarrow X / G, p_{X}: x \mapsto x G$.

Uma ação de $G$ sobre $X$ é chamada transitiva se $X / G$ consiste de uma única órbita.

Exemplo 2.1.7. O espaço das órbitas da ação à direita $G \times H \rightarrow G$ do Exemplo 2.1.4 é $G / H$, o espaço das classes laterais à direita $g H$. A função

$$
\begin{gathered}
G \times G / H \rightarrow G / H, \\
(g, k H) \mapsto g k H
\end{gathered}
$$

é contínua e, logo, a $G$-ação à esquerda sobre $G / H$. Qualquer $G$-espaço $G / H$ com essa ação é chamado um espaço homogêneo. 
Para cada $x \in X$, o conjunto $G_{x}=\{g \in G \mid g x=x\}$ é um subgrupo de $G$, o qual é chamado de grupo de isotropia de $x$ em $X$.

Definição 2.1.8. Suponha que $X$ e $Y$ sejam $G$ espaços. Uma aplicação $f: X \rightarrow Y$ é chamada $G$-equivariante ou uma $G$-aplicação se $f(x g)=f(x) g$ para todo $x \in X, g \in G$.

Uma $G$-aplicação $f: X \rightarrow Y$ induz uma aplicação quociente entre espaços de órbitas, o que pode ser representado pelo seguinte diagrama

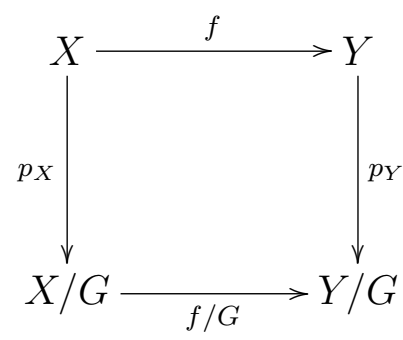

no qual as aplicações verticais são as aplicações quocientes da Definição 2.1.6.

A categoria de $G$-espaços e $G$-aplicações é denotada por $T o p_{G}$. Os isomorfismos nessa categoria são chamados G-homeomorfismos.

Definição 2.1.9. Seja $\phi: X \times G \rightarrow X$ uma ação livre. Considere $X^{*}=\{(x, x g) \mid x \in$ $X, g \in G\}$ (como um subespaço de $X \times X$ ) e defina a função

$$
\begin{gathered}
\tau_{X}: X^{*} \rightarrow G, \\
\tau_{X}:(x, x g) \mapsto g .
\end{gathered}
$$

Chamamos $\tau_{X}$ a função translação do $G$-espaço $X$.

A função translação satisfaz as seguintes propriedades:

(1) $\tau(x, x)=u_{G}$;

(2) $\tau\left(x, x^{\prime}\right) \tau\left(x^{\prime}, x\right)=u_{G}$;

(3) $\tau\left(x, x^{\prime}\right) \tau\left(x^{\prime}, x^{\prime \prime}\right)=\tau\left(x, x^{\prime \prime}\right)$;

(4) $x \tau\left(x, x^{\prime}\right)=x^{\prime}$.

A $G$-ação é chamada principal se $\tau$ é uma função contínua. Devemos também dizer que um $G$-espaço livre com uma ação principal é um $G$-espaço principal.

Ação principal é um tópico fundamental na definição de fibrados principais.

Exemplo 2.1.10. Considere $\mathbb{Z} / 2$ agindo sobre $S^{n}$ como no Exemplo 2.1 .18 mais a frente. Então, $\left(S^{n}\right)^{*}$ é o subespaço de $(x, \pm x) \in S^{n} \times S^{n}$ e a função translação é $\tau(x, \pm x)= \pm 1$, a qual é contínua.

Relembremos que uma função contínua $f: X \rightarrow Y$ é própria se, para cada espaço $Z$, a aplicação $f \times 1: X \times Z \rightarrow Y \times Z$ é fechada. Uma $G$-ação sobre um espaço $X$ é própria se a aplicação $\theta: X \times G \rightarrow X \times X, \theta:(x, g) \mapsto(x, x g)$ é uma aplicação própria. 
Proposição 2.1.11. Considere $G$ agindo livremente sobre $X$. Então $\tau_{X}$ é contínua se, e somente se, a ação de $G$ é própria.

A seguinte proposição é bastante usada quando tratamos de equivalências de fibrados.

Proposição 2.1.12. Sejam $X$ e $Y$ G-espaços livres, $f: X \rightarrow Y$ uma G-aplicação e considere o diagrama comutativo

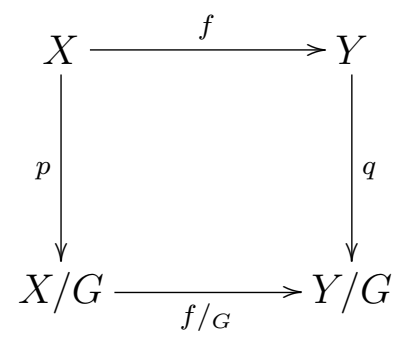

onde $f /{ }_{G}: X / G \rightarrow Y / G$ é um homeomorfismo e $p$ e q são as aplicações quocientes ( $p_{X} e$ $\left.p_{Y}\right)$. Suponha que $\tau_{Y}$ seja continua. Então $f$ é um G-homeomorfismo.

Demonstração: Como $f /{ }_{G}$ é homeomorfismo então $f$ é bijetiva sobre as órbitas. Vejamos que é bijetiva sobre as fibras.

Para verificarmos que é injetiva sobre a fibra de $x$, considere $x$ e $x^{\prime}$ nesta fibra tal que $f(x)=f\left(x^{\prime}\right)$. Temos $x^{\prime}=x g$ e então $f(x)=f\left(x^{\prime}\right)=f(x g)=f(x) g$. Como a ação é livre, $g=u$, isto é, $x=x^{\prime}$.

Vejamos que é sobrejetiva sobre a fibra de $y \in Y$. Como $f /{ }_{G}$ é sobrejetiva, existe $x \in X$ tal que $f /{ }_{G}(p(x))=q(y)$, isto é, pela comutatividade $q(f(x))=q(y)$, ou seja, $y$ e $f(x)$ estão na mesma fibra, $\operatorname{logo} f(x)=y g$ ou $f(x) g^{-1}=y$ ou $f\left(x g^{-1}\right)=y$ para algum $g \in G$. Assim, $f$ é uma bijeção contínua.

Resta mostrar que a função inversa é contínua: sejam $f(x)=y, U$ uma vizinhança aberta de $x$ e escolha vizinhanças abertas $V$ de $x$ e $N$ de $u$ tal que $V N \subset U$. Como $\tau_{Y}$ é contínua, existe uma vizinhança aberta $W$ de $y$ tal que $\tau_{Y}\left((W \times W) \cap Y^{*}\right) \subset N$. Já que $f$ é contínua, $V_{1}=V \cap f^{-1}(W)$ é uma vizinhança aberta de $x$ e $f\left(V_{1}\right) \subset W$. O conjunto $p\left(V_{1}\right)$ é uma vizinhança aberta de $p(x)$ pois $p$ é uma aplicação aberta. Seja $W_{1}=W \cap q^{-1}\left(p\left(V_{1}\right)\right)$. Então $W_{1}$ é uma vizinhança aberta de $y$ e $q\left(W_{1}\right)=p\left(V_{1}\right), f\left(V_{1}\right) \subset W_{1}$. Para $z \in W_{1}$, escolha $u \in V_{1}$ tal que $q(z)=p(u)$. Então, $f(u) \in W$ e $z \in W$. Qualquer $g \in G$ com $f(u) g=z$ está contido em $N$ e logo $z=f(u) g=f(u g) \operatorname{com} f^{-1}(z)=u g \in V_{1} N \subset U$. Conseqüentemente, $f^{-1}\left(W_{1}\right) \subset U$ e assim mostramos a continuidade de $f^{-1}$ em $z$.

Definição 2.1.13. Um G-espaço $X$ é chamado localmente trivial se cada ponto $x G$ do espaço de órbita $X / G$ tem uma vizinhança aberta $V$ tal que $p^{-1}(V)$ é G-homeomorfa a $V \times G$ (onde $p: X \rightarrow X / G$ é a projeção canônica da Definição 2.1.6).

No próximo lema veremos uma propriedade fundamental de espaços com uma $G$ aplicação tomando valores em $G$, bastante usada em trivialidade local de espaço homogêneos (ou trivialidade de fibrados). 
Lema 2.1.14. Seja $X$ um $G$-espaço e seja $f: X \rightarrow G$ uma $G$-aplicação. Então $X \cong$ $f^{-1}(u) \times G$.

Demonstração: Defina $\phi: f^{-1}(u) \times G \rightarrow X$ como $(x, g) \mapsto x g$. É imediato verificar $f \phi=p r_{2}$, a projeção natural $f^{-1}(u) \times G \rightarrow G$, e que $\phi$ é bijetiva. Além do mais, a aplicação $\psi: X \rightarrow f^{-1}(u) \times G, x \mapsto\left(x(f(x))^{-1}, f(x)\right)$ é uma inversa contínua de $\phi$.

Corolário 2.1.15. Se $X$ é um G-espaço livre e $f: X \rightarrow G$ e uma G-aplicação, então $X \cong X / G \times G$.

Note que o Lema 2.1.5 implica a seguinte relação de separabilidade em Top (fraca separabilidade em $k(T o p)$ ) do espaço de órbitas e do fêcho de $X^{*}: X / G$ é Hausdorff se, e somente se, $X^{*}$ é fechado em $X \times_{c} X$.

Como uma aplicação do lema 2.1.14, apresentamos uma condição suficiente para trivialidade local de um $G$-espaço principal.

Proposição 2.1.16. Seja $X$ um $G$-espaço principal com uma $G$-ação $\phi: X \times G \rightarrow X$. Suponha que exista um ponto $x_{0} \in X$ tal que $\left(X, x_{0} G\right)$ é uma $G$-co-fibração fechada. Então, a aplicação quociente $q: X \rightarrow X / G$ é localmente trivial.

Demonstração: Seja $\tau: X^{*} \rightarrow G$ a aplicação translação definida por $\phi$. Para todo $x \in X$, considere a restrição de $\tau$ ao subespaço $\{(x, x g) \mid g \in G\} \subset X^{*}$ a qual é um $G$-homeomorfismo com $G$; então $x G$ e $x_{0} G$ são $G$-homeomorfos como subespaços de $X$ e então $(X, x G)$ é uma $G$-co-fibração fechada. Seja $u_{x}: X \rightarrow I$ e $h_{x}: X \times I \rightarrow X$ as aplicações que definem $(X, x G)$ como um $G$-NDR. Considere a $G$-aplicação $f: U_{x} \rightarrow G$ definida por $f(x)=h(x, 1)$ para todo $x \in U_{x}$. Corolário 2.1.15 nos mostra que $U_{x}$ é $G$-homeomorfo a $U_{x} / G \times G$.

Essa proposição tem uma importante conseqüência: Seja $H$ um subgrupo fechado de $G \in T o p G r$. Se $(G, H)$ é uma $G$-co-fibração fechada, então $q: G \rightarrow G / H$ é localmente trivial. Esse é o caso de um subgrupo fechado de um grupo de Lie (grupos de Lie são CW-complexos, veja [28]).

Trivialidade local e ações triviais estão, de fato, estritamente relacionadas:

Proposição 2.1.17. Seja X um G-espaço livre. Suponha que X seja localmente trivial. Então $\tau_{X}$ é contínua.

Demonstração: Seja $U$ uma vizinhança $G$-equivariante de $x \in X$ a qual admite uma trivialização $\psi: V \times G \rightarrow U$. A pré-imagem de $(U \times U) \cap X^{*}$ segundo $\psi \times \psi$ é $\{(v, g, v, h) \mid v \in V, g, h \in G\}$ e $\tau_{X}(\psi \times \psi)$ é a aplicação $(v, g, v, h) \mapsto g^{-1} h$, que é contínua. 
Exemplo 2.1.18. Espaço projetivo real. Seja $\mathbb{Z} / 2$ o conjunto com dois elementos $\{0,1\}$ e a multiplicação de grupo: $0+0=1+1=0,0+1=1+0=1$. Esse é um grupo abeliano topológico com a topologia discreta (ele pode ser descrito também multiplicativamente pelo conjunto $\{ \pm 1\})$. Considere $\mathbb{Z} / 2$ agindo sobre $S^{n}=\left\{\left(x_{0}, \ldots, x_{n}\right) \mid \sum_{i=0}^{n} x_{i}^{2}=1\right\}$ por

$$
\left(z, x_{0}, \ldots, x_{n}\right) \mapsto(-1)^{z}\left(x_{0}, \ldots, x_{n}\right),
$$

(a ação antipodal). Então o espaço de órbitas dessa ação livre é chamado espaço projetivo real $\mathbb{R} P^{n}$ de dimensão $n$. Conceitualmente, $\mathbb{R} P^{n}$ é a variedade dos subespaços de dimensão 1 de $\mathbb{R}^{n+1}$, também denotado por $P\left(\mathbb{R}^{n+1}\right)$.

\section{$2.2 \quad$ Fibrados}

Agora que temos a teoria necessária para caracterizar um fibrado, podemos introduzir as seguintes definições:

\subsubsection{Definição global de um fibrado principal}

Definição 2.2.1. Um $G$-fibrado principal consiste de uma $G$ ação livre à direita $E \times G \rightarrow$ E e uma aplicação sobrejetiva $p: E \rightarrow B$ tal que:

(1) $p(e g)=p(e) g$

(2) para cada $b \in B$, existe uma vizinhança aberta $V$ de $b$ em $B$ e um G-homeomorfismo $\phi: p^{-1}(V) \rightarrow V \times G$ tal que

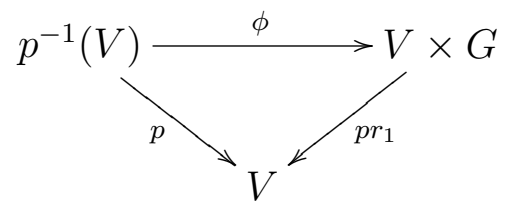

é comutativo.

O espaço $E$ na definição acima é chamado espaço total do fibrado, $B$ o espaço base e $\phi$ a trivialização sobre $V$.

A relação entre $G$-fibrados principais e $G$-espaços livres localmente triviais é clara:

Proposição 2.2.2. $(E, p, B, G)$ é um $G$-fibrado principal se, e somente se, E é um $G$ espaço localmente trivial e $B \cong E / G$.

\subsubsection{Fibrados associados}

Seja $p: E \rightarrow B$ um $G$-fibrado principal dado. Seja $F$ um espaço com uma $G$ ação efetiva à esquerda. Então podemos produzir um novo fibrado como segue. Considere $G$ agindo sobre o produto $E \times F$ por $(x, y) g=\left(x g, g^{-1} y\right)$, defina $E \times{ }_{G} F=(E \times F) / G$ e

$$
p_{F}: E \times_{G} F \rightarrow B=E / G,
$$




$$
[(x, y)] \mapsto p(x)
$$

$\left(E \times{ }_{G} F, p_{F}, B, G, F\right)$ é chamado fibrado com fibra $F$ e grupo $G$ sobre $B$ associado a $p$. Fibrados com fibra $\mathbb{R}^{n}, \mathbb{C}^{n}$ ou $\mathbb{H}^{n}$ são chamados de fibrados vetoriais real, complexo e quaterniônico, respectivamente. Quando $n=1$, são chamados fibrados linhas.

\subsubsection{Definição local de fibrados}

A definição dada acima para um $G$-fibrado principal é uma definição global. O espaço total tem uma ação expressa por uma função definida globalmente e a função translação está bem definida sobre o espaço todo (mesmo se não muito concreto). A existência de uma trivialização local está de alguma forma oculta. Existe uma definição equivalente de um $G$-fibrado principal, que considera o ponto de vista contrário, isto é, local e baseado sobre a introdução de um conjunto de funções locais. Essa é uma definição bastante usada em várias situações, abstratas e aplicadas, e em particular quando uma expressão explícita para essas funções locais está efetivamente disponível. Como essa definição aplica facilmente a uma classe grande de fibrados, preferimos apresentá-la no seguinte modo:

Definição 2.2.3. Um fibrado $\xi$ é uma quíntupla $(E, p, B, F, G)$ satisfazendo as seguintes propriedades:

(1) E, B, F são espaços topológicos, $p: E \rightarrow B$ é uma aplicação e $G$ é um grupo topológico agindo efetivamente à esquerda sobre $F$, isto é, $G \subseteq H o m e o(F)$;

(2) $B$ é coberto por uma coleção de conjuntos abertos $\mathcal{U}=\left\{U_{i}\right\}_{i \in J}$ e para todo $i \in J$ existe um homeomorfismo $\phi_{i}: U_{i} \times F \rightarrow p^{-1}\left(U_{i}\right)$ sobre $U_{i}$ (tal que $p \phi_{i}=p r_{1}$, onde $\operatorname{pr}_{1}: U_{i} \times F \rightarrow U_{i}$ é a projeção na primeira coordenada);

(3) para todo $b \in U_{i j}=U_{i} \cap U_{j} \neq \emptyset$ e todo $y \in F, \phi_{i}^{-1} \phi_{j}(b, y)=\left(b, g_{i j}(b) y\right)$ e a função $g_{i j}: U_{i j} \rightarrow G$ é contínua.

O espaço $E$ é o espaço total do fibrado $\xi, B$ é o espaço base, $F$ é a fibra e $G$ é o grupo estrutural. Finalmente, as aplicações $g_{i j}$ são suas funções transitivas. Um fibrado com grupo estrutural $G$ é também chamado um $G$-fibrado.

As funções-transição são os ingredientes chave para trabalhar com fibrados. A seguinte formulação de funções-transição é bastante usada: para todo $b \in U_{i j}$, defina

$$
\begin{gathered}
\phi_{i, b}:\{b\} \times F \rightarrow p^{-1}(b), \\
\phi_{i, b}(y)=\phi_{i}(b, y) ;
\end{gathered}
$$

então para todo $y \in F, g_{i j}(b) y=\phi_{i, b}^{-1} \phi_{j, b}(y)$.

É fácil verificar que as funções-transição satisfazem as seguintes condições:

$(F T 1) \forall b \in U_{i}, g_{i i}(g)=u$

$(F T 2) \forall b \in U_{i j},\left(g_{i j}(b)\right)^{-1}=g_{j i}(b)$, 
$(F T 3) \forall b \in U_{i j k}, g_{k i}(b)=g_{k j}(b) g_{j i}(b)$; a última condição pode ser escrita de um modo co-cíclico:

$$
g_{i j}(b) g_{j k}(b) g_{k i}(b)=u,
$$

para todo $i, j, k \in J$ e para todo $b \in U_{i j k}$.

A importância das funções-transição está também na seguinte proposição, cuja prova pode ser encontrada em [18].

Proposição 2.2.4. Seja $G$ um grupo topológico o qual age efetivamente à esquerda num espaço F. Seja B um espaço com uma cobertura aberta $\left\{U_{i}\right\}_{i \in J}$ e, para todo $i, j \in J$ para o qual $U_{i j} \neq \emptyset$, temos aplicações $g_{i j}: U_{i j} \rightarrow G$ as quais satisfazem as condições FT1, FT2, FT3. Então existe um fibrado $\xi=(E, p, B, F, G)$ com funções-transição $g_{i j}$.

Em particular, o resultado acima nos permite construir um $G$-fibrado com fibra $G$ fora de qualquer $G$-fibrado $\xi$ dado: apenas observe que $G$ age sobre si mesmo por multiplicação. Tal $G$-fibrado com fibra $G$ é o $G$-fibrado principal associado a $\xi$. Podemos agora mostrar que as duas definições dadas para um $G$-fibrado principal na verdade coincidem.

Proposição 2.2.5. O G-fibrado principal ( $E, p, B, F, G)$ mostrado na Definição 2.2.3 é um $G$-fibrado principal $(E, p, B, G)$ como na Definição 2.2.1 e a recíproca é verdadeira.

Demonstração: Por um lado, devemos mostrar que

(1) $E$ é um $G$-espaço principal;

(2) $B$ é homeomorfo ao espaço de órbitas $E / G$;

(3) o homeomorfismo local $\phi_{i}: U_{i} \times G \rightarrow p^{-1}\left(U_{i}\right)$ sobre os conjuntos abertos $U_{i}$ são $G$-equivariantes.

Podemos proceder como segue. A ação de $G$ sobre $E$ é dada por: se $x=\phi^{-1}\left(p(x), g_{i}\right)$ e $g \in G, x g=\phi_{i}\left(p(x), g_{i} g\right)$. A função translação $\tau: E^{*} \rightarrow G$ é então dada localmente como segue: se $x \in p^{-1}\left(U_{i}\right)$, defina $\sigma_{i}(x)=\operatorname{pr}_{2} \phi^{-1}(x) \in G$. Agora, defina $\tau_{i}\left(x, x^{\prime}\right)=\left(\sigma_{i}(x)\right)^{-1} \sigma_{i}\left(x^{\prime}\right)$, para todo $\left(x, x^{\prime}\right) \in p^{-1}\left(U_{i}\right)^{*}$. Claramente, se $x$ também pertence a $p^{-1}\left(U_{j}\right)$, então $\tau_{i}\left(x, x^{\prime}\right)=\tau_{j}\left(x, x^{\prime}\right)$. Assim a função global $\tau$ é contínua.

Falta provar que $\tau$ satisfaz a condição $x \tau\left(x, x^{\prime}\right)=x^{\prime}$, para todo $\left(x, x^{\prime}\right) \in E$. De fato,

$$
x \tau_{i}\left(x, x^{\prime}\right)=\phi_{i}\left(p(x), \sigma_{i}(x) \tau_{i}\left(x, x^{\prime}\right)\right)=\phi_{i}\left(p(x), \sigma_{i}\left(x^{\prime}\right)\right)=x^{\prime} .
$$

Reciprocamente, seja $E$ um $G$-espaço livre localmente trivial. Então, $E$ tem uma função translação contínua pela Proposicão 2.1 .17 e para provar que $\xi=$ $(E, p, B, F, G)$ é um $G$-fibrado principal é suficiente definir as funções transição. Considere as funções $\sigma_{i}: p^{-1}\left(U_{i}\right) \rightarrow G$ definidas como anteriormente e definamos

$$
g_{i j}: U_{i j} \rightarrow G
$$

por $g_{i j}(p(x))=\sigma_{i}(x)\left(\sigma_{j}(x)\right)^{-1}$. 
O estudo é mais simples quando consideramos a categoria dos $G$-fibrados principais, logo, em geral usaremos resultados para fibrados principais; tais resultados serão traduzidos imediatamente para os fibrados associados usando a construção acima.

Os objetos da categoria $\operatorname{Princ}_{G}(B)$ são $G$-fibrados principais sobre o espaço $B$. Um morfismo $f \in \operatorname{Princ}_{G}\left(\xi, \xi^{\prime}\right)$ é uma aplicação $G$-eqüivariante $f: E \rightarrow E^{\prime}$ a qual comuta com as projeções $p$ e $p^{\prime}$. Na verdade, qualquer morfismo $f$ será um $G$-homeomorfismo de $E$ em $E^{\prime}$ pela Proposição 2.1.12, e conseqüentemente um isomorfismo em $\operatorname{Princ}_{G}(B)$.

\subsubsection{Equivalência de fibrados}

Usando a definição global 2.2.1, é imediato introduzir a noção natural de equivalência de fibrados: isto é, isomorfismos na categoria $\operatorname{Princ}_{G}(B)$. $G$-fibrados principais isomorfos são também chamados equivalentes e essa é obviamente uma relação de equivalência. O mais interessante é como isso é traduzido na definição local, através das funções transição.

Proposição 2.2.6. Sejam $\xi$ e $\xi^{\prime}$ dois G-fibrados com a mesma base B e fibra F. E mais, suponha que ambos os fibrados sejam localmente triviais sobre a mesma cobertura aberta de $B$ (isso é sempre possivel simplesmente intersectando as coberturas abertas originais de ambos fibrados). Então $\xi$ e $\xi^{\prime}$ são equivalentes se, e somente se, para todo $i \in J$, existe uma aplicação $\rho_{i}: U_{i} \rightarrow G$ tal que

$$
g_{i j}^{\prime}(b)=\left(\rho_{j}(b)\right)^{-1} g_{i j}(b) \rho_{i}(b),
$$

para todo $b \in U_{i j}$.

Demonstração: Suponha que os fibrados sejam isomorfos e seja $f: E \rightarrow E^{\prime}$ o $G$ homeomorfismo sobre $B$. Para cada $i \in J$ defina

$$
\rho_{i}: U_{i} \rightarrow G
$$

por $\phi_{i}^{-1}(b, y)=\left(b, \rho_{i}(b) y\right)$. Então,

$$
\left(b, \rho_{j}(b) g_{j i}(b) y\right)=\left(b, g_{i j}^{\prime}(b) \rho_{j}(b) y\right),
$$

isto é, $g_{j i}^{\prime}(b)=\rho_{i}(b) \rho_{j}(b)^{-1}$ e as funções transição satisfazem a condição estabelecida na tese. Reciprocamente, suponha que as condições acima sejam verdadeiras, então construimos o homeomorfismo $f$ através das aplicações $\rho_{i}$ como segue. Seja

$$
f_{i j}(x)=\phi_{i}^{\prime}\left(b, \rho_{i}(b)^{-1} g_{i j}(b) y\right),
$$

$\operatorname{com} x=\phi(b, y)$. Para obter uma função globalmente definida, precisamos $f_{i j}=f_{k j}=f_{k h}$ sobre as intersecções $U_{i} \cap U_{k}$ e $U_{j} \cap U_{h}$. Mas

$$
\begin{gathered}
f_{i j}(x)=\phi_{k}^{\prime}\left(b, \rho_{k}(b)^{-1} g_{k h}(b) y^{\prime}\right)=\phi_{k}^{\prime}\left(b, \rho_{k}(b)^{-1} g_{k h}(b) g_{h j}(b) y\right)= \\
=\phi_{k}^{\prime}\left(b, \rho_{k}(b)^{-1} g_{k j}(b) \rho_{j}(b) y\right)=f_{k j}(x)=\phi_{i}^{\prime}\left(b, g_{i k}^{\prime}(b) \rho_{k}(b)^{-1} g_{k j}(b) y\right)=
\end{gathered}
$$




$$
=\phi_{i}^{\prime}\left(b, \rho_{i}(b)^{-1} g_{i j}(b) \rho_{j}(b) y\right)=f_{i j}(x),
$$

como desejado (ver também [18] 7.2.5, 7.2.7).

Introduzimos a notação $\xi \cong \xi^{\prime}$ para indicar que $\xi$ e $\xi^{\prime}$ são equivalentes. Temos que dois $G$-fibrados são equivalentes se, somente se, seus $G$-fibrados principais associados são equivalentes. No que segue, se nada contrário for explicitado, falaremos sobre fibrados querendo dizer classes de equivalência de fibrados, isto é, trabalharemos na categoria $P_{G}(B)$ das classes de equivalência dos $G$-fibrados principais.

\subsubsection{Seções e trivialidade}

A noção de trivialidade para fibrados é bem natural: o G-fibrado principal trivial é o espaço produto $B \times G$ (o fibrado trivial com fibra $F$ é $B \times F$ ), ou qualquer fibrado equivalente a ele.

A condição local para trivalidade é que as funções transição satisfazem a seguinte propriedade: para cada conjunto $U_{i}$ na cobertura aberta de $B$ existem funções

$$
\rho_{i}: U_{i} \rightarrow G
$$

tal que

$$
g_{i j}(b)=\rho_{i}(b)^{-1} \rho_{j}(b),
$$

para cada $b \in U_{i} \cap U_{j}$ (onde $\rho_{i}$ são as funções locais de uma equivalência como em 2.4).

Um critério bastante usado para estabelecer trivialidade de um fibrado utiliza a noção de seção.

Definição 2.2.7. Uma seção para um fibrado dado $p: E \rightarrow B$ (principal ou não) é uma inversa à esquerda da projeção, a saber, uma aplicação $s: B \rightarrow$ E tal que ps $=1_{B}$.

Denotaremos por $\Gamma(B, E)$ o conjunto de seções de um fibrado $p: E \rightarrow B$, ou o espaço associado com a topologia oportuna, como veremos mais a frente.

Para a prova da seguinte proposição ver [18] 4.8 .

Proposição 2.2.8. Um G-fibrado principal tem uma seção se, e somente se, ele é o fibrado trivial (a menos de equivalência de fibrados).

\subsubsection{Fibrado pull back induzido}

Agora, consideramos uma ferramenta bastante usada para produzir fibrados de um fibrado dado e uma aplicação dada em seu espaço base. 
Seja $p: E \rightarrow B$ um $G$-fibrado principal e $f: A \rightarrow B$ uma aplicação dada. Seja $Y$ o espaço pull back do seguinte diagrama:

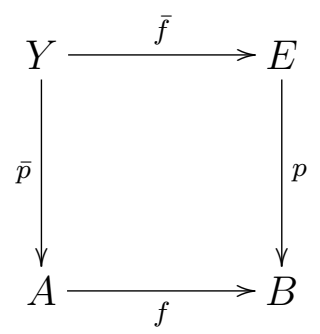

O que quer dizer, $Y$ é o subespaço $\{(a, x) \in A \times E \mid f(a)=p(x)\}$ de $A \times E$. Então $Y$ possui a $G$-ação induzida. Se $p$ é trivial sobre $V \subseteq B$, então $\bar{p}$ é trivial sobre $f^{-1}(V)$. Conseqüentemente, $(Y, q, A, G)$ é um $G$-fibrado principal. Ele é chamado o fibrado induzido por $f$ de $p$ e a notação $f^{-1}(p)$ (ou $f^{-1}(\xi)$, se $\xi$ é o fibrado $\left.p\right)$ é também usada.

Reciprocamente, assuma um diagrama comutativo

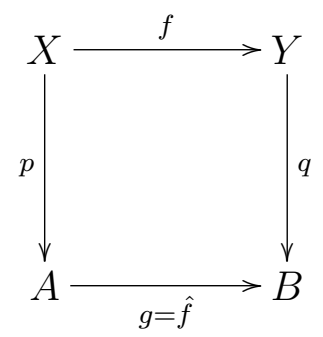

dado, com $G$-fibrados principais $p$ e $q$ e uma $G$-aplicação $f$. Então, $g=\hat{f}: X / G \cong A \rightarrow$ $Y / G \cong B$, a aplicação quociente induzida de $f$, e a aplicação $f$ é chamada uma aplicação fibrada. Seja $g^{-1}(q)$ o fibrado sobre $A$ induzido por $g$ de $q$. A propriedade universal do pull back produz uma aplicação $X \rightarrow g^{-1}(Y)$ (o espaço total de $g^{-1}(q)$ ) sobre $A$, a qual, pela Proposição 2.1.12, é um G-homeomorfismo. Logo, temos:

Proposição 2.2.9. Dados dois G-fibrados principais $p: X \rightarrow A$ e $q: Y \rightarrow B$ e uma aplicação fibrada $f: X \rightarrow Y$, então o fibrado $p$ é canonicamente isomorfo ao fibrado induzido $\hat{f}^{-1}(q)$.

\subsubsection{Somando fibrados}

O conceito de soma de Whitney de fibrados vetoriais se generaliza a $G$-fibrados principais como segue. Seja $\left\{\xi_{k}\right\}_{k=1, \ldots, n}$ o conjunto de $G_{k}$-fibrados principais sobre $B$. Suponha que $\mathcal{U}=\left\{U_{i}\right\}_{i \in J}$ seja uma cobertura aberta de $B$ sobre a qual os $\xi_{k}$ s são todos localmente triviais. Finalmente, seja $\left\{g_{i j}^{k}\right\}_{i, j \in J}$ o conjunto de funções transição de $\xi_{k}$. As funções transição

$$
\bigoplus_{k=1}^{n} g_{i j}^{k}: U_{i j} \rightarrow \bigoplus_{k=1}^{n} G_{k}
$$

definem um $\bigoplus_{k=1}^{n} G_{k}$-fibrado principal sobre $B$ o qual denotamos por $\bigoplus_{k=1}^{n} \xi_{k}$ e chamamos soma de $\xi_{1}, \ldots, \xi_{n}$. 
No caso de $k=2$, temos o fibrado pull back da aplicação diagonal $\Delta: B \rightarrow B \times B$, obtido do diagrama

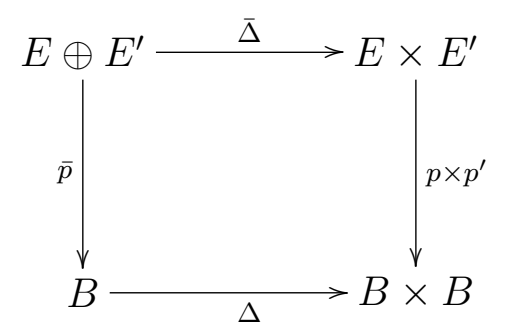

Já que a fibra é $\bar{p}^{-1}(b)=p^{-1}(b) \times p^{-1}(b)$, o produto das fibras, é também chamado produto de fibras.

\subsubsection{Exemplos de fibrados}

Exemplo 2.2.10. Provavelmente o exemplo mais fácil de um fibrado é a Faixa de Möbius. Ela pode ser descrita como segue. A ação de $\mathbb{Z} / 2=\{ \pm 1\}$ sobre $S^{1},(x, \pm 1) \mapsto \pm x$, é livre e principal. Então, temos o $\mathbb{Z} / 2$-fibrado principal $\xi=\left(S^{1}, p, \mathbb{R} P^{1}, \mathbb{Z} / 2\right)$, com projeção $p(x)=[ \pm x]$. Agora, como $\mathbb{R} P^{1} \cong S^{1}$, o espaço total desse fibrado é o bordo da Faixa de Möebius e o fibrado associado com fibra o intervalo unitário, é exatamente a Faixa de Möebius.

Exemplo 2.2.11. Pelo Exemplo 2.1.10, a projeção $p: S^{n} \rightarrow \mathbb{R} P^{n}$ define um $\mathbb{Z} / 2$-fibrado principal.

Exemplo 2.2.12. Fibrados tangente e normal à esfera. O fibrado tangente à $S^{n}$, denotado por $\tau\left(S^{n}\right)=\left(T S^{n}, p, S^{n}, \mathbb{R}^{n}, O_{n}(\mathbb{R})\right)$, e o fibrado normal por $\nu\left(S^{n}\right)=$ $\left(N S^{n}, q, S^{n}, \mathbb{R}^{n}, O_{n}(\mathbb{R})\right)$ são os fibrados vetoriais cujos espaços totais podem ser descritos como os seguintes subespaços do espaço produto $S^{n} \times \mathbb{R}^{n+1}$

$$
\begin{gathered}
T S^{n}=\left\{(b, x) \in S^{n} \times \mathbb{R}^{n+1} \mid\langle b, x\rangle=0\right\}, \\
N S^{n}=\left\{(b, x) \in S^{n} \times \mathbb{R}^{n+1} \mid x=\alpha b, \alpha \in \mathbb{R}\right\} .
\end{gathered}
$$

Um elemento $(b, x)$ de $T S^{n}$ é chamado um vetor tangente à $S^{n}$ em $b$ e um elemento de $N S^{n}$ e chamado vetor normal à $S^{n}$ em $b$. As fibras $p^{-1}(b)$ e $q^{-1}$ são espaços vetoriais de dimensão $n$ e 1, respectivamente. Uma seção de $\tau\left(S^{n}\right)$ é chamada um campo vetorial tangente sobre $S^{n}$ e uma seção de $\nu\left(S^{n}\right)$ um campo vetorial normal sobre $S^{n}$.

Exemplo 2.2.13. Para cada $b \in \mathbb{R}^{n}, b \neq 0$, existem duas funções lineares: a projeção sobre $b$

$$
\begin{gathered}
\pi_{b}: \mathbb{R}^{n} \rightarrow \mathbb{R}, \\
\pi_{b}: x \mapsto \frac{\langle x, b\rangle}{\langle b, b\rangle},
\end{gathered}
$$

e a aplicação normal

$$
\nu_{b} ; \mathbb{R}_{n} \rightarrow \mathbb{R}^{n}
$$




$$
\nu_{b}: x \mapsto x-\frac{\langle x, b\rangle}{\langle b, b\rangle} b,
$$

tal que $x=\nu_{b}(x)+\pi_{b}(x) x$ e $\left\langle b, \nu_{b}(x)\right\rangle=0$ para cada $x \in \mathbb{R}^{n}$.

Agora considere os dois fibrados $\tau\left(S^{n}\right)$ e $\nu\left(S^{n}\right)$ definidos no exemplo anterior. Podemos provar que a soma dos dois é trivial, mais precisamente considere $\theta_{n}(B)$ o fibrado vetorial trivial de posto $n$ sobre $B$, com espaço total $B \times \mathbb{R}^{n}$. Então

$$
\tau\left(S^{n}\right) \oplus \nu\left(S^{n}\right) \cong \theta_{n+1}\left(S^{n}\right) .
$$

Para isso, usamos o morfismo

$$
\begin{gathered}
u: E\left(\tau\left(S^{n}\right) \oplus \nu\left(S^{n}\right)\right) \rightarrow B \times \mathbb{R}^{n+1}, \\
u:\left((b, x),\left(b, x^{\prime}\right)\right) \mapsto\left(b, x+x^{\prime}\right),
\end{gathered}
$$

no qual usamos o fato que o espaço total da soma é o subespaço de $T S^{n} \times N S^{n}$.

Isso ocorre sobre a base $S^{n}$ e tem uma inversa

$$
v:(b, x) \mapsto\left(\left(b, \nu_{b}(x)\right),\left(b, \pi_{b}(x) b\right)\right) .
$$

Os elementos de $T S^{n}$ são chamados vetores tangentes sobre $S^{n}$, a fibra sobre $x \in S^{n}$ é $p^{-1}(x)=T S_{x}^{n}$, o espaço vetorial de todos os vetores tangentes à $S^{n}$ em $x$.

Como a matriz Jacobiana é ortogonal, as funções-transição tomam valores no grupo ortogonal e, logo, $\tau\left(S^{n}\right)$ é um $O_{n}(\mathbb{R})$-fibrado e seu fibrado principal associado é um $O_{n}(\mathbb{R})$ fibrado principal.

Uma seção $s: S^{n} \rightarrow \tau\left(S^{n}\right)$ é chamada um campo vetorial sobre $S^{n}$.

\subsection{Classificação de homotopia de $G$-fibrados principais}

O objetivo desta seção é mostrar como o processo de classificar fibrados é feito na teoria de homotopia. Não é possível afirmar um resultado completamente geral, mas nos restringimos a alguns tipos de fibrados (fibrados vetoriais podem ser uma possível escolha). A escolha mais geral é provavelmente tratar dos fibrados enumeráveis e isso conduz ao Teorema Fundamental de Classificação 2.3.2. abaixo.

Precisamos primeiro introduzir o conceito de fibrado enumerável e também algumas noções de teoria de homotopia relacionadas a fibrados. Não provaremos o Teorema de Classificação, tentaremos dar as ferramentas principais e suas propriedades sem provas, com o único propósito de dar uma idéia de como esse procedimento de classificar objetos funciona.

Primeiro recorde que, se $X$ é um espaço de Hausdorff, uma família $\left\{u_{i}\right\}_{i \in J}$ de aplicações $u_{i}: X \rightarrow I$ é chamada uma partição da unidade sobre $X$ se:

(1) os suportes das $u_{i}$ formam uma cobertura fechada de vizinhanças finitas de $X$,

(2) $\sum_{i \in J} u_{i}(x)=1$, para todo $x \in X$. 
Uma cobertura aberta $\left\{u_{i}\right\}_{i \in J}$ de um espaço topológico $B$ é enumerável se existe uma partição da unidade (localmente finita) $\left\{u_{i}\right\}_{i \in J}$ tal que $\overline{u_{i}^{-1}((0,1])} \subseteq U_{i}$ para cada $i \in J$.

Um resultado padrão é que um espaço de Hausdorff $B$ é paracompacto se, e somente, cada cobertura aberta é enumerável.

Um $G$-fibrado principal $\xi$ sobre um espaço $B$ é enumerável se é localmente trivial sobre uma cobertura aberta enumerável de $B$.

Observe que cada $G$-fibrado principal sobre um espaço paracompacto é enumerável.

Recorde que Htop denota a categoria cujos objetos são espaços topológicos e os morfismos são classes de equivalência de aplicações homotópicas.

Para cada espaço $B$ existe um funtor contravariante

$$
[-, B]: \text { Htop } \rightarrow \text { Set }
$$

para a categoria de conjuntos, o qual transforma um espaço $X$ no conjunto de classes de equivalência das aplicações homotópicas $[X, B]$, e morfismos $f \in H t o p(X, Y)$ na função

$$
\begin{gathered}
f^{*}:[X, B] \rightarrow[Y, B], \\
f^{*}:[h] \mapsto[h f] .
\end{gathered}
$$

A seguinte proposição é fundamental quando falamos de fibrados e sua prova pode ser encotrada em [18] 4.9.9:

Proposição 2.3.1. Sejam $p: E \rightarrow B$ um $G$-fibrado principal enumerável $e f, g: A \rightarrow B$ aplicações dadas. Então, $f \sim g$ implica $f^{-1}(p) \cong g^{-1}(p)$.

Essa proposição nos permite introduzir o funtor contravariante

$$
N P_{G}: \text { Htop } \rightarrow \text { Set }
$$

para a categoria dos conjuntos, o qual transforma um espaço $B$ no conjunto $N P_{G}(B)$ (o fato que fibrados são caracterizados pelas funções-transição garante que $N P_{G}(B)$ é, de fato, um conjunto) de todas as classes de equivalência dos $G$-fibrados principais enumeráveis sobre $B$ e o morfismo $[f] \in \operatorname{HTop}(A, B)$ na função

$$
\begin{gathered}
N P_{G}([f]): N P_{G}(B) \rightarrow N P_{G}(B), \\
N P_{G}([f]): \xi \mapsto f^{-1}(\xi) .
\end{gathered}
$$

Agora, seja $\tilde{\xi}=(\tilde{E}, \tilde{p}, \tilde{B}, G)$ um $G$-fibrado principal enumerável fixado. Podemos ver que existe uma transformação natural

$$
\mathcal{T}:[-, \tilde{B}] \rightarrow N P_{G}
$$

tal que, para todo $B \in H T o p$,

$$
\mathcal{T}(B):[B, \tilde{B}] \mapsto N P_{G}(B), \quad[f] \mapsto f^{-1}(\tilde{\xi}) .
$$


Podemos enunciar agora o seguinte Teorema de Classificação:

Teorema 2.3.2. Se Ẽ é contrátil, os funtores $N P_{G}$ e $[-, \tilde{B}]$ de HTop em Set são naturalmente equivalentes.

Demonstração: Ver [18] 4.10, 4.12.

Um $G$-fibrado principal enumerável com espaço total contrátil é chamado G-fibrado universal. O espaço base de um $G$-fibrado universal é o espaço classificante de $G$. Existem vários modos para construir um espaço classificante e essas construções são todas functoriais. A construção de Milgram-Steenrod de um fibrado universal $\xi_{G}=\left(E G, p_{G}, B G, G\right)$, para qualquer grupo topológico $G$, está descrita em [39]. A construção original foi feita por Milnor [18] 4.11.

Pelo Teorema 2.3.2 temos que, quando um $G$-fibrado universal é dado, para cada espaço $B$ existe uma bijeção entre os conjuntos

$$
\mathcal{T}:[B, \tilde{B}] \cong N P_{G}(B)
$$

Essa é a classificação de homotopia do $G$-fibrados principais enumeráveis.

Isso significa que, quando um certo fibrado é suficientemente trivial segundo o ponto de vista homotópico, então ele pode ser usado para classificar todos os fibrados do mesmo tipo. A necessidade de contratibilidade pode ser enfraquecida quando desejamos classificar fibrados, a menos de alguma dimensão fixada. De fato, temo o seguinte:

Proposição 2.3.3. Se $\tilde{E}$ é n-conexo (isto é, os n primeiros grupos de homotopia são triviais), então os functores $N P_{G} e[-, \tilde{B}]$ são naturalmente equivalentes na categoria de $C W$-complexos de dimensão no máximo $n$.

Um $G$-fibrado principal com espaço total $n$-conexo é chamado $n$-fibrado universal (para tais fibrados não é necessário ser enumerável, já que todo $C W$-complexo de dimensão finita é paracompacto). Veja também [33] II.6 e [6].

A maioria dos fibrados encontrados neste capítulo têm uma base paracompacta. Assim, eles são enumeráveis e, a menos que tenhamos enumerabilidade por diferentes razões, não faremos referência explicitamente a enumerabilidade e assumiremos que os fibrados principais são enumeráveis.

\subsection{Grupos de gauge}

Mais referências para esta seção estão em [38] e [18].

O grupo de gauge de um fibrado principal é simplesmente seu grupo de automorfismos com a topologia vinda da topologia do espaço de aplicações (a topologia compacto aberta).

Definição 2.4.1. Seja $p: E \rightarrow B$ um $G$-fibrado principal. O grupo de gauge $\mathcal{G}_{p}$ é o subespaço dos $G$-homeomorfismos de E sobre $B$. 
Explicitamente, $\mathcal{G}_{p}$ é o conjunto das aplicações $u \in m(E, E)$, i. e., $u: E \rightarrow E$, tal que

$$
p u=p,
$$

e

$$
u(x g)=u(x) g,
$$

para todo $g \in G, x \in E$. De fato, $G$-equivariância sobre $B$ garante que $u \in \operatorname{Homeo}(E)$ pela Proposição 2.1.12.

Isso significa que a multiplicação natural no conjunto $\mathcal{G}_{p}$, a saber, composição de aplicações, tem uma inversa e como é associativa e tem a aplicação identidade como unidade natural, temos para $\mathcal{G}_{p}$ a estrutura natural de um grupo abstrato.

Por outro lado, $\mathcal{G}_{p}$ é um subconjunto do conjunto $m(E, E)$, que pode ser considerado um espaço usando a topologia compacto aberta e, assim, $\mathcal{G}_{p}$ pode ser visto como um subespaço com a topologia induzida. Uma questão que surge é: as estruturas algébricas e topológicas são compatíveis, ou seja, $\mathcal{G}_{p}$ é um grupo topológico? Sabemos que a composição é contínua, mas há a questão referente à continuidade de $u \mapsto u^{-1}$. É verdade que $\mathcal{G}_{p}$ é um grupo topológico, mas é necessário um pouco mais de trabalho para provar.

Por completamento, mostramos aqui uma situação um pouco geral quando isso é verdade ([11] 1.1.22).

Proposição 2.4.2. Se X é um espaço de Hausdorff compacto, então Homeo $(X)$ é um grupo topológico na topologia compacto aberta.

Para provar o mesmo resultado de uma maneira geral, necessário quando estudamos grupos de gauge, primeiro precisamos introduzir algumas formulações diferentes para o próprio grupo de gauge.

\section{Formulação 1}

Recorde que, para um grupo topológico $G$, denotamos por $A d(G)$ o $G$-espaço à direita $G$ com $G$-ação adjunta à direita dada por $a d:(x, g) \mapsto g^{-1} x g$ (veja Exemplo 2.1.4). Também, $G$ é um $G$-grupo, já que a estrutura de grupo topológico sobre $G$ é preservada pela $G$-ação à direita.

Para $X$ e $Y$ dois $G$-espaços à direita, denotamos por $m_{G}(X, Y)$ o subespaço das $G$ aplicações, isto é, $f \in m(X, Y)$ satisfazendo

$$
f(x g)=f(x) g
$$

para todo $g \in G, x \in X$. Em particular, $\mathcal{G}_{p}$ é um subespaço de $m_{G}(E, E)$.

Considere o conjunto $m_{G}(E, A d(G))$ e dê a ele a topologia herdada de $m(E, A d(G))$, e a multiplicação

$$
(f, g) \mapsto f g, \quad f g(x)=f(x) g(x),
$$

para todo $x \in X$, o que quer dizer que a aplicação produto é definida como o produto das imagens usando a multiplicação em $A d(G)$. Isso faz de $m(E, A d(G))$ um grupo topológico. 
Agora, considere a função

$$
\begin{gathered}
\alpha: \mathcal{G}_{p} \rightarrow m(E, A d(G)), \\
\alpha: u \mapsto \phi_{u}
\end{gathered}
$$

na qual $\phi_{u}(x)=\tau(x, u(x))$. A aplicação $\alpha$ é uma bijeção contínua. E mais, a função

$$
\begin{gathered}
\beta: m(E, A d(G)) \rightarrow \mathcal{G}_{p}, \\
\beta: \phi \rightarrow u_{\phi},
\end{gathered}
$$

sendo $u_{\phi}(x)=x \phi(x)$, é uma inversa contínua para $\alpha$, e logo, estabelecemos um homeomorfismo $\mathcal{G}_{p} \cong m(E, A d(G))$. Como em $G$ a inversão $g \rightarrow g^{-1}$ é contínua, em $m(E, A d(G))$ temos que a inversão também é contínua, $\operatorname{logo} m(E, A d(G))$ é também grupo topológico.

Usando essa descrição pode-se provar o seguinte:

Proposição 2.4.3. Seja $p: E \rightarrow B$ um G-fibrado principal. Se pé trivial ou se $G$ é abeliano, então $\mathcal{G}_{p}$ é isomorfo, como um grupo topológico, a $m(B, G)$.

Demonstração: ([18] 2.1.7) No primeiro caso, $m_{G}(B \times G, A d(G))=m(B, G)$ já que $f(b, g)$ com $f(b, g h)=b^{-1} f(b, g) h$ é da forma $f(b, g)=g^{-1} f(b, u) g$ para todo $g \in G$ e $b \in B$. No segundo caso, $m_{G}(E, A d(G))=m(B, G)$ pois $f(x g)=f(x)$, já que $G$ é abeliano.

\section{Formulação 2}

A próxima formulação para o grupo de gauge está em termos das seções de um fibrado. Dado um $G$-fibrado principal $p: E \rightarrow B$, considere o fibrado associado $E \times{ }_{G} A d(G)$. Esse é um fibrado de grupos, já que a estrutura de grupo sobre $A d(G)$ é $G$-equivariante, e o espaço das seções $\Gamma\left(B, E \times{ }_{G} A d(G)\right)$ é um grupo topológico em que multiplicamos seções usando o produto em $A d(G)$.

Existe um modo importante para descrever seções de um fibrado. A saber, existe uma função

$$
\begin{aligned}
\mu: m(E, A d(G)) & \rightarrow \Gamma\left(B, E \times_{G} A d(G)\right), \\
\mu: u & \mapsto s_{u},
\end{aligned}
$$

sendo $s_{u}(b)=(x, u(x)) G$, para $x \in F_{b}$, um elemento arbitrário da fibra $F_{b}$ sobre $b \in B$. A aplicação $\mu$ é uma bijeção, e logo, temos a terceira descrição do grupo de gauge.

\section{Formulação 3}

A última descrição é uma descrição local, em termos das funções-transição. Essa descrição é particularmente importante na teoria de gauge e aplicações à física. Assuma 
que o $G$-fibrado $\xi, p: E \rightarrow B$, é localmente trivial sobre a cobertura aberta $\mathcal{U}=\left\{U_{i}\right\}_{i \in J}$ de $B$ e tem funções transição $g_{i j}$.

Começamos definindo o grupo de gauge local de $p$ associado a cobertura aberta $\mathcal{U}$ como o grupo topológico

$$
\mathcal{L}(p)=\prod_{i \in J} m\left(U_{i}, G\right)
$$

dotado com a topologia produto. Agora, para todo $i \in J$ defina $\xi_{i}$ a restrição de $\xi$ a $U_{i}$. Como $\xi_{i}$ é trivial, o grupo de gauge $\mathcal{G}_{\xi_{i}}$ é homeomorfo ao grupo topológico $m\left(U_{i}, G\right)$, via função

$$
\begin{gathered}
\vartheta_{i}: \mathcal{G}_{\xi_{i}} \rightarrow m\left(U_{i}, G\right), \\
\vartheta_{i}: f_{i} \mapsto \vartheta_{i}\left(f_{i}\right),
\end{gathered}
$$

em que $\vartheta_{i}\left(f_{i}\right)(b)=\left(\phi_{i, b}^{-1} f_{i, b} \phi_{i, b}\right)(u)$, para toda $f_{i} \in \mathcal{G}_{\xi_{i}}$ e todo $b \in U_{i}$ e sendo $f_{i, b}$ a restrição de $f_{i}$ à fibra $p^{-1}(b)$.

Agora, definamos a aplicação

$$
\begin{gathered}
r_{i}: \mathcal{G}_{\xi} \rightarrow \mathcal{G}_{\xi_{i}}, \\
r_{i}:\left.f \mapsto f\right|_{\left(p^{-1}\left(U_{i}\right)\right)} .
\end{gathered}
$$

Lema 2.4.4. A função

$$
\begin{gathered}
\vartheta: \mathcal{G}_{\xi} \rightarrow \mathcal{L}(p), \\
\vartheta: f \mapsto\left\{\vartheta_{i} r_{i}(f)\right\}_{i \in J},
\end{gathered}
$$

é um mergulho de grupos topológicos.

Identificando $\mathcal{G}_{p} \operatorname{com} \vartheta\left(\mathcal{G}_{\xi}\right) \subset \mathcal{L}(p)$ podemos caracterizar $\mathcal{G}_{p}$ usando as funções transição de $\xi$ :

Proposição 2.4.5. O grupo $\mathcal{G}_{p}$ coincide com o subgrupo

$$
\left\{\left\{f_{i}\right\}_{i \in J} \in \mathcal{L}(p) \mid f_{j}=g_{i j}^{-1} f_{i} g_{i j} \text { sobre } U_{i j}\right\}
$$

As provas do Lema e da Proposição acima podem ser encontrados em [34].

Como conseqüências do teorema anterior, podemos ver mais uma vez que se $G$ é abeliano, o grupo de gauge $\mathcal{G}_{p}$ coincide com $m(B, G)$.

Podemos ver que se $\xi \cong \xi^{\prime}$, então $\mathcal{G}_{\xi} \cong \mathcal{G}_{\xi^{\prime}}$. De fato, seja $\ell: E \rightarrow E^{\prime}$ um homeomorfismo $G$-eqüivariante sobre $B$. Então

$$
\begin{gathered}
A d_{\ell}: \mathcal{G}_{\xi} \rightarrow \mathcal{G}_{\xi^{\prime}}, \\
A d_{\ell}: f \mapsto \ell^{-1} f \ell
\end{gathered}
$$

é um isomorfismo. Assim, faz sentido falar sobre o grupo de gauge de um fibrado querendo dizer uma classe de equivalência de fibrados. No entanto, observe que o contrário não é verdade: o $\mathbb{Z}$-fibrado trivial $S^{1} \times \mathbb{Z} \rightarrow S^{1}$ e a aplicação fibrado exponencial $e^{2 \pi i}: \mathbb{R} \rightarrow S^{1}$ não são equivalentes, mas têm grupos de gauge homeomorfos. 
Observação 2.4.6. Para qualquer conjunto finito de $G$-fibrados principais sobre um espaço dado $B$, podemos selecionar um único grupo de gauge local comum de tal modo que os teoremas de mergulho acima continuam verdadeiros para os grupos de gauge de todos os fibrados envolvidos.

Fechamos essa seção apresentando dois resultados: sobre os grupos de gauge de uma soma de fibrados e sobre o centro do grupo de gauge.

Primeiro, considere a soma de fibrados principais, como descrito na seção 2.7.

Proposição 2.4.7. Para qualquer conjunto de $G_{k}$-fibrados principais $\xi_{1}, \ldots, \xi_{n}$ sobre $B$,

$$
\mathcal{G}_{\bigoplus_{k=1}^{n} \xi_{k}} \cong \bigoplus_{k=1}^{n} \mathcal{G}_{\xi_{k}} .
$$

Agora, considere o centro do grupo de gauge de um $G$-fibrado principal $\xi=(E, p, B, G)$. Para continuar nossa análise, precisamos introduzir a seguinte condição: $[C 1] \eta: \mathcal{G}_{\xi} \rightarrow G, \eta:\left.f \mapsto f\right|_{p^{-1}\left(b_{0}\right)\left(u_{g}\right)}$, é uma sobrejeção para todo $b_{0} \in B$.

Então, considere o homeomorfismo

$$
m_{G}(E, A d(G)) \rightarrow \mathcal{G}_{\xi}
$$

introduzido na formulação 2. Por outro lado, observamos que essa aplicação preserva o centro e assim

$$
Z m_{G}(E, A d(G)) \cong Z \mathcal{G}_{\xi} .
$$

Por outro lado, $u \in Z m_{G}(E, A d(G))$ se, e somente se, $f(x) u(x)=u(x) f(x)$ para toda $f \in m_{G}(E, A d(G))$ e para todo $x \in E$. Assim, se $[C 1]$ está satisfeita, $u \in$ $Z m_{G}(E, Z A d(G)) \cong m(B, Z G)$ já que $Z G$ é abeliano. Logo, temos o seguinte homeomorfismo:

Proposição 2.4.8.

$$
Z \mathcal{G}_{\xi} \cong m(B, Z G)
$$

\subsubsection{A homotopia do grupo de gauge}

Seja $p: E \rightarrow B$ um $G$-fibrado principal e $p_{G}: E G \rightarrow B G$ um $G$-fibrado universal. O objetivo desta seção é apresentar alguma informação sobre o tipo de homotopia do grupo de gauge de $p$. Para isso, mostraremos como introduzir um espaço classificante para o grupo de gauge. Desse espaço classificante e seu fibrado universal, informações sobre o tipo de homotopia do grupo de gauge podem ser deduzidas por métodos-padrão da teoria de homotopia. Mais precisamente, apresentaremos duas possíveis abordagens para o problema de introduzir um espaço classificante para o grupo de gauge $\mathcal{G}_{p}$.

\section{Formulação 1}


Recorde que $m_{G}(E, E G)$ é o subespaço das aplicações $G$-equivariantes em $m(E, E G)$. Agora, o Teorema de Classificação 2.3.2 implica que existe uma aplicação $f: B \rightarrow B G$ tal que $p$ é (equivalente a) o fibrado pull back $f^{-1}\left(p_{G}\right)$ induzido pelo diagrama

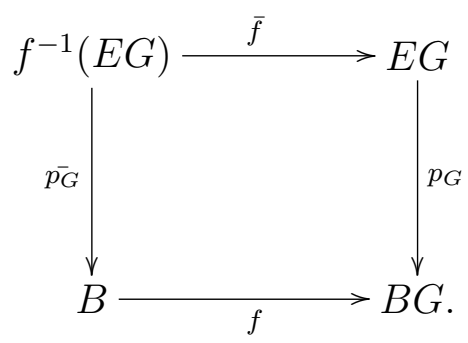

Seja $m(B, B G ; f)$ a componente conexa de $m(B, B G)$ a qual contém a aplicação $f$. Como $g^{-1}\left(p_{G}\right)=f^{-1}\left(p_{G}\right)$ para toda $g \sim f$, todas as aplicações em $m(B, B G ; f)$ definem o mesmo fibrado $p$ pelo pull back.

Seja

$$
\begin{aligned}
q: m(E, E G) & \rightarrow m(B, B G), \\
q: w & \mapsto w / G,
\end{aligned}
$$

a aplicação quociente que associa a cada $w \in m(E, E G)$ a classe de equivalência de $w$, $w / G \in m(B, B G)$.

Sabemos que o grupo de gauge $\mathcal{G}_{p}$ é um grupo topológico. E mais, age pela direita sobre o espaço $m_{G}(E, E G)$ pela composição de morfismos de fibrados, a saber:

$$
\begin{gathered}
\phi: m_{G}(E, E G) \times \mathcal{G}_{p} \rightarrow m_{G}(E, E G), \\
\phi:(w, u) \mapsto w u,
\end{gathered}
$$

e é uma ação contínua. Também, se $w, w^{\prime} \in m_{G}(E, E G)$ com $q(w)=q\left(w^{\prime}\right)$, então temos $\tau\left(w, w^{\prime}\right)=w^{-1} w^{\prime} \in \mathcal{G}_{p} \operatorname{com} w \tau\left(w, w^{\prime}\right)=w^{\prime}$, a qual é uma função translação. Essa função

$$
\tau:\left(m_{G}(E, E G)\right)^{*} \rightarrow \mathcal{G}_{p}
$$

é contínua (funções composição e inversa). Provamos então o seguinte:

Proposição 2.4.9. Sejam $p: E \rightarrow B$ um G-fibrado principal e $p_{G}: E G \rightarrow B G$ um $G$-fibrado universal. Então, o espaço $m_{G}(E, E G)$ é um $\mathcal{G}_{p}$-espaço principal, com projeção $q: m_{G}(E, E G) \rightarrow m(B, B G ; f)$. O fibrado q é chamado fibrado principal padrão para o grupo de gauge $\mathcal{G}_{p}$.

Observe que não é garantida a trivialidade local.

O interesse nessa definição segue de (veja [18] 4.3.4, 4.3.5):

Proposição 2.4.10. Se o espaço total de $p: E \rightarrow B$ é localmente compacto, então o fibrado principal padrão para $\mathcal{G}_{p}$ é contrátil e, conseqüentemente, o espaço classificante para o grupo de gauge $\mathcal{G}_{p}$ é $m(B, B G ; f)$, sendo o fibrado universal o fibrado principal padrão. 


\section{Formulação 2}

A segunda abordagem é mais utilizada para estudar o tipo de homotopia do grupo de gauge.

Seja $G$ um grupo topológico e seja $p: E \rightarrow B$ um $G$-fibrado principal sobre $B$. Sejam $B_{G}$ o espaço classificante de $G$, e $p_{G}: E G \rightarrow B G$ o fibrado universal. Seja $f$ a aplicação classificante para $p$, isto é, tal que o diagrama

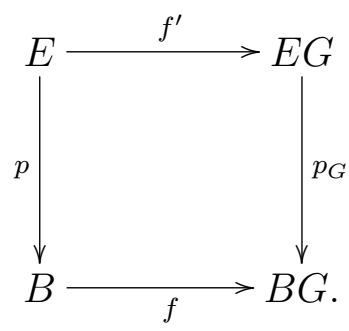

comuta (a menos de isomorfismo).

Considere o grupo $G$ com a $G$-ação à esquerda $a d_{g}\left(g^{\prime}\right)=g^{-1} g^{\prime} g$. Esse é o $G$-grupo à esquerda $A d(G)$ e dá origem a dois fibrados $q: E \times_{G} A d(G) \rightarrow B, q_{G}: E G \times_{G} A d(G) \rightarrow$ $B G$. Se $B$ é paracompacto, esses fibrados são fibrações.

E mais, o seguinte diagrama comuta

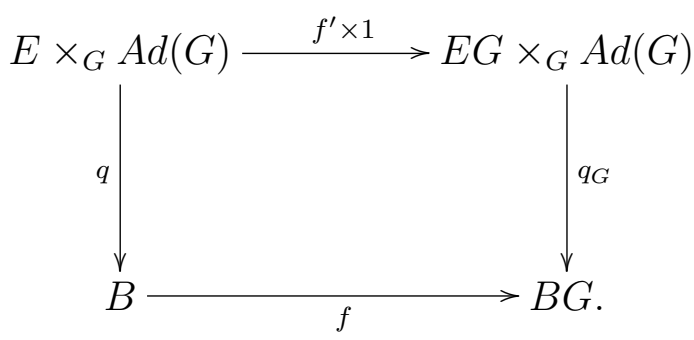

e novamente é um pull back a menos de isomorfismo de fibrados.

Se $B$ é localmente compacto $q_{G *}: m\left(B, E G \times_{G} A d(G)\right) \rightarrow m(B, B G)$ é uma fibração e o diagrama

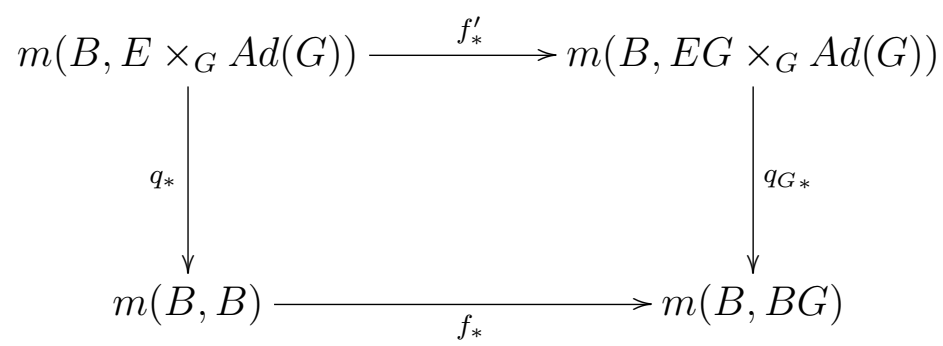

é um pull back (novamente, a menos de isomorfismo). Logo, $q_{*}$ é uma fibração e sua fibra sobre $\left\{1_{B}\right\}$ é, na verdade, o grupo de gauge de $p$. De fato,

$$
q_{*}^{-1}\left(1_{B}\right)=\left\{s \in m\left(B, E \times_{G} A d(G)\right) \mid q s=1_{B}\right\}=\Gamma\left(B, E \times_{G} A d(G)\right) .
$$

Isso significa que as fibras sobre todas as funções nas componentes conexas por caminhos de $f$ têm o mesmo tipo de homotopia de $\mathcal{G}(p)$, como esperado. Mas, como todas as 
aplicações em tais componentes conexas por caminhos são homotópicas, temos que isso significa que essas fibras são isomorfas.

Já que é um diagrama pull back, temos também que a fibra de $q_{G_{*}}$ é o grupo de gauge $\mathcal{G}_{p}$ e assim, obtemos a fibração

$$
\mathcal{G}_{p} \rightarrow m\left(B, E G \times_{G} A d(G)\right) \rightarrow m(B, B G),
$$

segundo suposições adequadas sobre $B$ e $G$. O tipo de suposições necessárias para obter resultados mais úteis têm sido estudado por vários autores. Um cenário bem geral está descrito nos trabalhos de Booth, Heat, Morgan e Piccinini (veja, por exemplo, [5]). Traduzimos aqui alguns de seus resultados na linguagem de fibrados.

Proposição 2.4.11. Seja $p: E \rightarrow B$ um G-fibrado principal classificado por uma aplicação $f: B \rightarrow B G$. Então, o grupo de gauge $\mathcal{G}_{p}$ tem o mesmo tipo de homotopia do espaço dos laços $\Omega m(B, B G ; f)$. E mais, essa é uma equivalência de H-espaços.

Proposição 2.4.12. Seja $p: E \rightarrow B$ um G-fibrado principal classificado por uma aplicação $f: B \rightarrow B G$. Suponha que $B$ tem o tipo de homotopia de um $C W$-complexo finito e que $G$ é um $C W$-complexo. Então, o espaço classificante para o grupo de gauge $B \mathcal{G}_{p}$ tem o mesmo tipo de homotopia do espaço das aplicações $m(B, B G ; f)$.

Demonstração: [38] 2.3.

\subsection{O espaço dos laços sobre o espaço classificante de um grupo topológico}

Seja $G$ um grupo topológico conexo compacto. Seja $p_{G}: E G \rightarrow B G$ seu fibrado universal como construído em [38]. Obtemos uma equivalência de $H$-espaços $\Omega B G \sim_{H} G$.

Lema 2.5.1. $G \sim L_{p_{G}}$.

Demonstração: $\mathrm{O}$ espaço $L=L_{p_{G}}$ é definido como o pull back do seguinte diagrama [37]

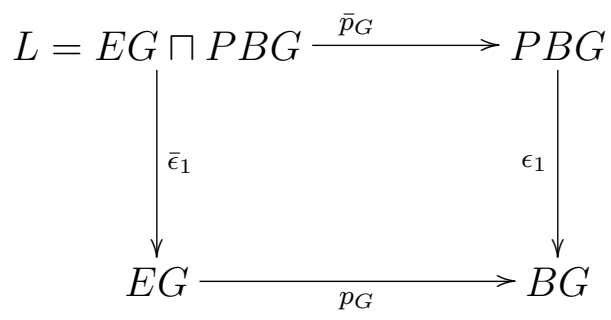

no qual $P B G=\left\{\lambda \in m(I, B G) \mid \lambda(0)=b_{0}\right\}, b_{0}$ é o ponto base de $B G$ e

$$
\epsilon_{1}: \lambda \mapsto \lambda(1)
$$

e, então, $L=\left\{(y, \gamma) \in E G \times P B G \mid \epsilon_{1}(\gamma)=\gamma(1)=p_{G}(y)\right\}$. 
Considere a aplicação

$$
\begin{gathered}
h: G \rightarrow L, \\
h: g \mapsto\left((g, 1), c_{b_{0}}\right) ;
\end{gathered}
$$

e está bem definida já que $p_{G}(g, 1)=[(g, 1)]=b_{0}=\epsilon_{1}\left(c_{b_{0}}\right)=c_{b_{0}}(1)$.

Considere a homotopia

$$
\begin{gathered}
H: L \times I \rightarrow B G, \\
H:((y, \gamma), t) \mapsto \gamma(1-t) .
\end{gathered}
$$

Então o diagrama

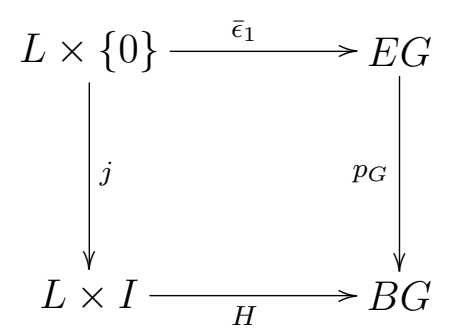

comuta, já que $H((y, \gamma), 0)=\gamma(1)=\epsilon_{1}(\gamma)$. Logo, existe uma homotopia

$$
\hat{H}: L \times I \rightarrow E G,
$$

tal que $p_{G} \hat{H}=H$ e $\left.\hat{H}\right|_{L \times\{0\}}=\bar{\epsilon}_{1}$. Agora, $p_{G} \hat{H}((y, \gamma), 1)=H((y, \gamma), 1)=\gamma(0)=b_{0}($ já que $\gamma \in P B G)$. Assim, $\hat{H}((y, \gamma), 1) \in p_{G}^{-1}\left(b_{0}\right)=G$. Definimos

$$
\begin{gathered}
k: L \rightarrow G, \\
k:(y, \gamma) \mapsto \hat{H}((y, \gamma), 1) .
\end{gathered}
$$

Podemos provar que $h k \sim_{F} 1_{L}$ e $k h \sim_{K} 1_{G}$, sendo

$$
\begin{gathered}
F: L \times I \rightarrow L, \\
F:((y, \gamma), t) \mapsto\left((\hat{H}((y, \gamma), 1), 1-t), \gamma_{t}\right),
\end{gathered}
$$

em que $\gamma_{t}(s)=\gamma(t s)$

$$
\begin{gathered}
K: G \times I \rightarrow G, \\
K:(g, t) \mapsto \hat{H}\left(\left((g, 1), c_{b_{0}}\right), t\right) .
\end{gathered}
$$

Lema 2.5.2. $L \sim \Omega B G$.

Demonstração: Consideremos as duas aplicações

$$
\begin{gathered}
f_{1}: L=E G+P B G \rightarrow \Omega B G, \\
f_{1}:(y, \gamma) \mapsto \gamma+p_{G} \lambda_{y}^{-1},
\end{gathered}
$$


tendo-se $y=\left(g_{1}, t_{1}\right) \cdots\left(g_{n}, t_{n}\right)(g, 1)$, definimos o caminho

$$
\lambda_{y}(t)=\left(g_{1}, t_{1} t\right) \cdots\left(g_{n}, t_{n} t\right)(g, t) .
$$

Então, $\lambda_{y}(0)=y_{0}, \lambda_{y}(1)=y$, o que implica $p_{G}\left(\lambda_{y}(1)\right)=p_{G}(y)=\gamma(1)$, isto é, o caminho $p_{G} \lambda_{y}$ termina onde o caminho $\gamma$ termina. Como $\lambda^{-1}(s)=\lambda(1-s)$, temos

$$
\begin{aligned}
\left(\gamma+p_{G} \lambda_{y}^{-1}\right)(t)= \begin{cases}\gamma(2 t) & 0 \leq t \leq \frac{1}{2} \\
p_{G} \lambda^{-1}(2 t-1)=p_{G} \lambda(2-2 t) & \frac{1}{2} \leq t \leq 1\end{cases} \\
f_{2}: \Omega B G \rightarrow L \\
f_{2}: \alpha \mapsto\left(y_{0}, \alpha\right) .
\end{aligned}
$$

Agora,

$$
f_{1} f_{2}: \alpha \mapsto\left(y_{0}, \alpha\right) \mapsto \alpha+p_{G} \lambda_{y_{0}}^{-1}=\alpha+c_{b_{0}} ;
$$

Primeiro, provamos que $f_{1} f_{2} \sim_{H_{1}} 1_{\Omega B G}$, em que

$$
\begin{gathered}
H_{1}: \Omega B G \times I \rightarrow \Omega B G, \\
H_{1}:(\alpha, s) \mapsto \alpha_{s}, \\
\alpha_{s}(t)= \begin{cases}\alpha\left(\frac{2 t}{s+1}\right) & 0 \leq t \leq \frac{s+1}{2}, \\
b_{0} & \frac{s+1}{2} \leq t \leq 1 .\end{cases}
\end{gathered}
$$

De fato, $H_{1}(\alpha, 0)=\alpha_{0}=\alpha+c_{b_{0}}$ e $H_{1}(\alpha, 1)=\alpha_{1}=\alpha$ já que:

$$
\begin{aligned}
& \alpha_{0}(t)= \begin{cases}\alpha(2 t) & 0 \leq t \leq \frac{1}{2} \\
b_{0} & \frac{1}{2} \leq t \leq 1\end{cases} \\
& \alpha_{1}(t)= \begin{cases}\alpha(t) & 0 \leq t \leq 1 \\
b_{0} & 1 \leq t \leq 1\end{cases}
\end{aligned}
$$

Podemos verificar também que $\alpha_{s} \in \Omega B G$, ou seja, $\alpha_{s}(0)=\alpha_{s}(1)=b_{0}$. Isso segue da definição de $\alpha_{s}$.

Continuando,

$$
f_{2} f_{1}:(y, \gamma) \mapsto \gamma+p_{G} \lambda_{y}^{-1} \mapsto\left(y_{0}, \gamma+p_{G} \lambda_{y}^{-1}\right)
$$

e provamos que $f_{2} f_{1} \sim_{H_{2}} 1_{L}$,

$$
\begin{gathered}
H_{2}: L \times I \rightarrow L, \\
H_{2}:((y, \gamma), s) \mapsto\left(\lambda_{y}\left(\frac{2 s}{s+1}\right), \lambda_{s}\right),
\end{gathered}
$$

sendo

$$
\lambda_{s}(t)=\left\{\begin{array}{ll}
\gamma\left(\frac{2 t}{s+1}\right) & 0 \leq t \leq \frac{s+1}{2}, \\
p_{G} \lambda_{y}^{-1}\left(\left(\frac{2 t}{s+1}-1\right)\right)=p_{G} \lambda_{y}\left(2-\frac{2 t}{s+1}\right) & \frac{s+1}{2} \leq t \leq 1 .
\end{array},\right.
$$

o que está bem definido já que em $t=\frac{s+1}{2}$ temos $\gamma(1)$.

De fato, $H_{2}((y, \gamma), 0)=\lambda_{0}=\gamma+p_{G} \lambda_{y}^{-1}$ e $H_{2}((y, \gamma), 1)=\lambda_{1}=(y, \gamma)$ já que:

$$
\lambda_{0}(t)= \begin{cases}\gamma(2 t) & 0 \leq t \leq \frac{1}{2} \\ p_{G} \lambda_{y}^{-1}(2 t-1) & \frac{1}{2} \leq t \leq 1\end{cases}
$$




$$
\lambda_{1}(t)= \begin{cases}\gamma(t) & 0 \leq t \leq 1 \\ p_{G} \lambda_{y}^{-1}(t-1)=p_{G}\left(\lambda_{y}^{-1}(0)\right)=p_{G}\left(\lambda_{y}(1)\right)=p_{G}(y)=\gamma(1) & 1 \leq t \leq 1 .\end{cases}
$$

Nos resta verificar que $H_{2}((y, \gamma), s) \in L$ para todo $s$, o que significa

$$
p_{G} \lambda_{y}\left(\frac{2 s}{2+1}\right)=\epsilon_{1}\left(\lambda_{s}\right)=\lambda_{s}(1)
$$

e, de fato,

$$
\lambda_{s}(1)=p_{G} \lambda_{y}\left(2-\frac{2}{s+1}\right)=p_{G} \lambda_{y}\left(\frac{2 s}{s+1}\right) .
$$

Resumindo, provamos que a aplicação

$$
\begin{gathered}
f=f_{1} h: G \rightarrow \Omega B G \\
f: g \mapsto\left((g, 1), c_{b_{0}}\right) \mapsto c_{b_{0}}+p_{G} \lambda_{(g, 1)}^{-1}=c_{b_{0}}+[(g, t)]
\end{gathered}
$$

é uma equivalência de homotopia de $G$ em $\Omega B G$. 


\subsection{Apêndice do capítulo 2}

Faremos aqui uma breve recordação de alguma noções básicas sobre categorias e funtores, e apresentaremos algumas definições necessárias para a compreensão da categoria dos $k$-espaços Hausdorff fraco.

Definição 2.6.1. A categoria $\mathcal{C}$ consiste em

- um conjunto de objetos: $X, Y, Z, \ldots$;

- um conjunto de morfismos: $f: X \rightarrow Y, g: Y \rightarrow Z, \ldots$;

- uma lei de composição de morfismos: dados quaisquer dois morfismos, $f: X \rightarrow Y$ e $g: Y \rightarrow Z$, existe um morfismo $f g: X \rightarrow Z$, satisfazendo as seguintes regras:

- associatividade: para cada morfismo, $f, g, h$ temos $f(g h)=(f g) h$;

- unidade: para cada objeto $X$, existe um morfismo identidade $1_{X}: X \rightarrow X$, tal que para qualquer morfismo $f: X \rightarrow Y, f 1_{X}=1_{Y} f=f$.

Definição 2.6.2. Um funtor covariante entre duas categorias $\mathcal{F}: \mathcal{C} \rightarrow \mathcal{D}$ é uma lei que associa a cada objeto $X$ e a cada morfismo $f: X \rightarrow Y$ em $\mathcal{C}$, um objeto $\mathcal{F}(\mathcal{C})$ e um morfismo $\mathcal{F}(f): \mathcal{F}(X) \rightarrow \mathcal{F}(Y)$ em $\mathcal{D}$, satisfazendo as seguintes regras:

- $\mathcal{F}\left(1_{X}\right)=1_{\mathcal{F}(X)}$,

- $\mathcal{F}(f g)=\mathcal{F}(f) \mathcal{F}(g)$.

A partir dessas definições vamos descrever as categorias que foram necessárias para desenvolver este capítulo, e apresentar algumas das importantes propriedades relacionadas a elas. Começamos com uma lista parcial de categorias que devemos usar.

Set - categoria dos conjuntos e funções entre conjuntos:

Gr - categoria dos grupos e homomorfismos de grupo;

Top - categoria de todos os espaços topológicos (chamados apenas de espaços) e funções contínuas (chamadas aplicações);

CW - categoria dos $C W$-complexos e aplicações (não necessariamente celular);

HTop - categoria dos espaços e classes de homotopia de aplicações. Essa é a categoria de homotopia associada a Top;

Se considerarmos apenas os objetos baseados nas categorias acima (isto é, cada objeto é considerado junto com um elemento distingüido: um ponto base) e considerarmos apenas os morfismos que levam pontos base em pontos base, então temos subcategorias Set $_{0}, G r_{0}$, Top $_{0}, C W_{0}$; para HTop, obtemos HTop por homotopias baseadas.

Uma questão de notação: para qualquer categoria $\mathcal{C}$, denotamos por $\mathcal{C}(X, Y)$ o conjunto de todos os morfismos de um objeto $X$ em um objeto $Y$ de $\mathcal{C}$.

Podemos combinar algumas dessas categorias, por exemplo, podemos considerar a categoria TopGr de grupos topológicos, seus morfismos são homomofismos de grupos contínuos.

Em particular, falaremos mais da categoria $w H k(T o p)$ dos $k$-espaços Hausdorff fraco, a qual é usada por razões técnicas e que precisa de algumas explicações. De fato, $w H k(T o p)$ contém todos os espaços 'bons' que tratamos neste trabalho - como as variedades suaves - e logo, numa primeira leitura, àqueles que não são familiares com $k$-espaços Hausdorff fraco 
podem simplesmente ignorar sua definição e admitir que estão trabalhando em espaços 'bons'.

Definimos $k$-espaços Hausdorff fraco como segue: seja $X$ um espaço dado. Um subconjunto $A \subset X$ é compactamente fechado se, para todo espaço de Hausdorff compacto $K$ e toda aplicação $f: K \rightarrow X, f^{-1}(A) \subset K$ é fechado em $K$. O espaço $X$ é chamado um $k$-espaço se todos os seus subconjuntos compactamente fechado são fechados. Agora defina um funtor $k:$ Top $\rightarrow$ Top associando a cada $X \in$ Top o espaço $k(X)$ com o mesmo conjunto latente que $X$ mas com a topologia considerando como conjuntos fechados os conjuntos campactamente fechados com respeito a topologia de $X$. Quanto aos morfismos, observamos que $Y$ é um $k$-espaço, então uma função $f: Y \rightarrow X$ é uma aplicação se, e somente se, $f: Y \rightarrow k(X)$ é contínua. Assim, para qualquer morfismo $f \in \operatorname{Top}(Y, X)$, simplesmente consideramos $k(f)=f: k(Y) \rightarrow k(X)$. O funtor $k$ é chamado $k$-ificação e sua imagem $k(T o p)$ é toda uma subcategoria de Top. A categoria $k(T o p)$ é completa e co-completa, com o produto de $k$-espaços dados por

$$
X \times Y=k\left(X \times_{c} Y\right)
$$

sendo $X \times{ }_{c} Y$ o produto cartesiano usual em Top. Um $k$-espaço $X$ é chamado Hausdorff fraco sempre que a diagonal $\Delta_{X}: X \rightarrow X \times X$ é fechada em $X \times X$. A categoria $w H k(T o p)$ é toda subcategoria de Top determinada por todos os $k$-espaços Hausdorff fraco e aplicações. Existe uma caracterização bastante usada de $k$-espaços Hausdorff fracos: se $X$ é um $k$-espaço, então $X$ é Hausdorff fraco se, e somente se, para toda aplicação $f: K \rightarrow X$, com $K$ compacto e Hausdorff, $f(K)$ é fechado e Hausdorff compacto.

Vejamos agora as principais propriedades de $w H k(T o p)$. Para começar, observamos que a caracterização acima de $k$-espaços Hausdorff fraco implica que esses espaços têm a propriedade de separação $T_{1}$. Eles não são necessariamente Hausdorff assim como a propriedade de $\Delta$ ser fechada em $k(T o p)$ não é tão forte quanto ser fechada em Top (existem exemplos de $k$-espaços que não são Hausdorff fraco: Tychonoff plank é um exemplo, veja [13] Apêndice 1).

Agora, $w H k(T o p)$ é fechado segundo a formação de subespaços, produto finito e coprodutos e quociente por subespaços fechados. A categoria $w H k(T o p)$ tem espaço de aplicações (definido por $Y^{X}=k(m(X, Y))$, com $m(X, Y)$ dotado da topologia compacto aberta) e isso satisfaz a lei exponencial

$$
\left(Z^{X}\right)^{Y} \cong Z^{X \times Y}
$$

Finalmente, $w H k(T o p)$ é fechado segundo a formação de espaços adjuntos: dado que $A$ é um subespaço fechado de $X$, para qualquer aplicação $f: A \rightarrow B$, o espaço $B \sqcup_{f} X$ obtido como um push out do diagrama $X \leftarrow A \rightarrow B$ é também um objeto de $w H k(T o p)$.

Como fizemos antes, podemos combinar a categoria $G r$ com $w H k(T o p)$ e obter a categoria $w H k(T o p) G r$ de grupos topológicos Hausdorff fraco.

Um espaço $X \in$ Top tem a topologia inicial induzida por uma aplicação $i: X \rightarrow Y$ se, e somente se, para todo $Z \in T o p, f \in T o p(Z, X)$ temos $f \in T o p(Z, Y)$. Dualmente, um espaço $X$ tem a topologia final induzida por uma aplicação $p: Y \rightarrow X$ se, e somente se, 
para todo espaço $Z \in T o p, g \in T o p(X, Z)$ temos $g p \in T o p(Y, Z)$. Se $X$ tem a topologia inicial induzida por $i: X \rightarrow Y$ em Top e $i$ é injetiva, dizemos que $i$ é uma inclusão, então $U \subset X$ é aberto se, e somente se, $i(U) \subset i(X)$ é aberto (observando também que uma injeção de Top é fechada se, e somente se, é uma inclusão fechada). Se $X$ tem a tologia final induzida por $p: Y \rightarrow X$ e $p$ é sobrejetiva, dizemos que $p$ é uma proclusão. Então $U \subset X$ é aberto se, e somente se, $p^{-1}(U) \subset Y$ é aberto. A composição de duas inclusões (respectivamente proclusões) é uma inclusão (respectivamente proclusão). E mais, um produto finito de inclusões em Top é uma inclusão ([4] 1.4.1). A propriedade correspondente para proclusões não é verdade em Top, mas é em $w H k(T o p)$ :

Lema 2.6.3. Suponha que $f: X \rightarrow Y$ e $f^{\prime}: X^{\prime} \rightarrow Y^{\prime}$ sejam proclusões em $w H k(T o p)$. Então $f \times f^{\prime}: X \times X^{\prime} \rightarrow Y \times Y^{\prime}$ é uma proclusão em $w H k(T o p)$.

Demonstração: Como o funtor $X \times$ - preserva co-limites [13] o espaço $X \times Y^{\prime}$ tem a topologia final com respeito a $1_{X} \times f^{\prime}$. Um argumento similar aplicado a $-\times Y^{\prime}$ mostra que $X^{\prime} \times Y^{\prime}$ tem a topologia final com respeito a $f \times f^{\prime}$.

Temos também a seguinte propriedade do funtor $k$ :

Lema 2.6.4. O funtor $k$-ificação $k:$ Top $\rightarrow$ Top preserva inclusões.

Demonstração: Seja $i \in T o p(X, Y)$ uma inclusão. Claramente, $k i$ é injetiva. Agora, mostraremos que, dado qualquer $Z \in k(T o p)$, uma função $g: Z \rightarrow k(X)$ é contínua se, e somente se, $k i g$ é contínua. De fato, kig é contínua se, e somente se, $i g: Z \rightarrow Y$ é contínua se, e somente se, $g: Z \rightarrow X$ é contínua se, e somente se, $g: Z \rightarrow k(X)$ é contínua.

Recordamos agora a definição e as principais propriedades da seqüência expandida de espaços em $w H k(T o p)$. Para mais detalhes, ver [13]. Uma seqüência expandida de espaços em $w H k(T o p)$ é uma seqüência $\left\{X_{n}, n \in \mathbb{N}\right\}$ de espaços em $w H k(T o p)$ tal que, para cada $n \in \mathbb{N}, X_{n}$ é um subespaço fechado de $X_{n+1}$. O espaço união da seqüência expandida é o conjunto $X=\bigcup_{n=0}^{\infty} X_{n}$ dotado com a topologia final com respeito a família de inclusões $X_{n} \subset X$. A família de espaços $\left\{X_{n}, n \in \mathbb{N}\right\}$ é também chamada uma filtração de $X$. Uma aplicação $f$ entre dois espaço filtrados $X$ e $Y$ é dita filtrada se existe uma seqüência de aplicações $f_{n}: X_{n} \rightarrow Y_{n}$ a qual é compatível com as filtrações, isto é, se $\left.f_{n+1}\right|_{X_{n}}=f_{n}$, para todo $n \in \mathbb{N}$. Claramente, uma seqüência compatível de aplicações induz uma aplicação (a aplicação união) entre espaços união.

As propriedades princiais do espaço união de uma seqüência expandida são devido ao fato de que a topologia do espaço união é coerente com a determinada pela família $\left\{X_{n}, n \in \mathbb{N}\right\}$ e cada $X_{n}$ é um subconjunto fechado de $X$. Em particular, isso implica que $w H k(T o p)$ é fechado segundo a formação de espaços união de seqüências expandidas. E mais, se todas as inclusões $X_{n} \subset X_{n+1}$ são co-fibrações fechadas, então as inclusões $X_{n} \subset X$ também são co-fibações fechadas.

Os seguintes lemas mostram como o processo de considerar a aplicação união de uma sequência compatível entre espaços filtrados preserva inclusões fechadas, proclusões e cofibrações fechadas. 
Lema 2.6.5. Sejam $X=\bigcup_{n=0}^{\infty} X_{n}$ e $Y=\bigcup_{n=0}^{\infty} Y_{n}$ espaços filtrados, e seja $f_{n}: X_{n} \rightarrow Y_{n}$ uma sequência compativel de inclusões fechadas (respectivamente proclusões). Então, a aplicação união f é uma inclusão fechada (respectivamente proclusão).

Demonstração: Começamos com inclusões fechadas. Como $f$ é injetiva, temos apenas que provar que $f$ é fechada. Seja $C$ fechado em $X$. Então $f(C) \cap Y_{n}=f_{n}\left(C \cap X_{n}\right)$ é fechado em $Y_{n}$, e logo, em $Y$. Segue que $f(C)$ é fechado em $Y$.

Vejamos agora as proclusões. Vemos que $f$ é sobrejetiva. Nos resta mostrar que $Y$ tem a topologia final co-induzida por $f$. Se $V \subseteq Y$ é tal que $f^{-1}(V)$ é aberto em $X$, então $f^{-1}(V) \cap X_{n}$ é aberto em $X_{n}$, para cada $n$. Agora considere $V_{n}=V \cap Y_{n}$ e observe que $f^{-1}(V)=\bigcup_{n=0}^{\infty} f^{-1}\left(V_{n}\right)$ e

$$
f^{-1}(V) \cap X_{n}=f_{n}^{-1}\left(V_{n}\right)
$$

é aberto em $X_{n}$. Como cada $f_{n}$ é uma proclusão, $V_{n}$ é aberto em $Y_{n}$ para cada $n$ e assim, $V$ é aberto em $Y$.

Para a preservação de co-fibrações, precisamos de uma suposição a mais sobre as aplicações envolvidas:

Lema 2.6.6. Sejam $X=\bigcup_{n=0}^{\infty} X_{n}$ e $Y=\bigcup_{n=0}^{\infty} Y_{n}$ espaços filtrados, e seja $f_{n}: X_{n} \rightarrow$ $Y_{n}$ uma sequência compativel de co-fibrações fechadas. Suponha também que todas as inclusões $X_{n} \subset X_{n+1}$ e $Y_{n} \subset Y_{n+1}$ sejam co-fibrações fechadas. Então a aplicação união $f: X \rightarrow Y$ é uma co-fibração fechada.

A prova segue na mesma linha de [13] A.5.5 (o requisito de normalidade não é necessário). 


\section{Capítulo}

3

\section{Métodos de composição dos grupos de homotopia de esferas}

Um dos principais problemas em teoria de homotopia é determinar os grupos de homotopia de esferas, já que essa é a primeira dificuldade fundamental nos cálculos dos grupos de homotopia de poliedros e espaços topológicos. Faremos um estudo dos grupos de esferas baseados em [46], onde a teoria mais detalhada e provas dos resultados apresentados podem ser encontrados.

Um exemplo de grupos não triviais aparece em [16], no qual temos o homomorfismo de Hopf $H$ de $\pi_{2 n-1}\left(S^{n}\right)$ no grupo dos inteiros $\mathbb{Z}$. Se $n$ é par, então a imagem $H\left(\pi_{2 n-1}\left(S^{n}\right)\right)$ do homomorfismo de Hopf contém $2 \mathbb{Z}$, em particular, se $n=2,4$ ou 8 então $H$ é sobrejetiva e a aplicação de fibra de Hopf $h: S^{2 n-1} \rightarrow S^{n}$ tem invariante de Hopf 1, isto é, $H(h)=1$.

O primeiro exemplo de um grupo finito não trivial foi feito por Freudenthal em [12]: $\pi_{n+1}\left(S^{n}\right) \approx \mathbb{Z} / 2$ para $n \geq 3$. Em [12], o homomorfismo suspensão de Freudenthal

$$
\Sigma: \pi_{i}\left(S^{n}\right) \rightarrow \pi_{i+1}\left(S^{n+1}\right)
$$

foi definido e teve um importante papel. O grupo $\pi_{3}\left(S^{2}\right)$ é cíclico infinito e é gerado pela classe $\eta_{2}$ da aplicação de Hopf $: S^{3} \rightarrow S^{2}$. O gerador do grupo $\pi_{n+1}\left(S^{n}\right)$ é $\eta_{n}=\Sigma^{n-2} \eta_{2}$, onde $\Sigma^{n-2}$ é a $(n-2)$ iteração do homomorfismo suspensão $\Sigma$. O homomorfismo $\Sigma$ é um isomorfismo se $i<2 n-1$ e disso obtemos o conceito de grupo estável

$$
G_{k}=\lim _{n \rightarrow \infty} \pi_{n+k}\left(S^{n}\right)
$$

o qual é isomorfo a $\pi_{n+k}\left(S^{n}\right)$ se $n>k+1$.

A segunda seqüência de grupos $\pi_{n+2}\left(S^{n}\right) \approx \mathbb{Z} / 2, n \geq 2$, foi determinada por G. Whitehead [48], e o gerador de $\pi_{n+2}\left(S^{n}\right)$ é a composição $\eta_{n} \circ \eta_{n+1}$. Em geral, o operador composição

$$
\circ: \pi_{j}\left(S^{i}\right) \times \pi_{i}\left(S^{n}\right) \rightarrow \pi_{j}\left(S^{n}\right)
$$

está definido simplesmente considerando a classe de homotopia da composição $g \circ f$ : $S^{j} \rightarrow S^{i} \rightarrow S^{n}$ das aplicações $f$ e $g$.

Vemos nesses exemplos, junto com o isomorfismo

$$
\pi_{i-1}\left(S^{n-1}\right)+\pi_{i}\left(S^{2 n-1}\right) \rightarrow \pi_{i}\left(S^{n}\right), n=2,4,8
$$


dado pela correspondência $(\alpha, \beta) \mapsto \Sigma \alpha+\gamma \beta$ em que $\gamma$ é o homomorfismo induzido da aplicação de fibra de Hopf $h$ (ver [43]), que o homomorfismo suspensão e o operador composição são ferramentas fundamentais para calcular os geradores dos grupos de homotopia das esferas.

\subsection{O produto de Whitehead}

Antes de continuarmos nossos cálculos sobre os grupos de homotopia das esferas, recordemos algums conceitos básicos do produto de Whitehead. Para maiores detalhes e provas dos resultados ver [47] Cap. X pág. 472.

Sejam $\alpha \in \pi_{p+1}(X), \beta \in \pi_{q+1}(X)$, representados pelas aplicações $f:\left(E_{1}, \dot{E}_{1}\right) \rightarrow$ $(X, *), g:\left(E_{2}, \dot{E}_{2}\right) \rightarrow(X, *)$, sendo $E_{1}, E_{2}$ células orientadas de dimensão $p+1, q+1$, respectivamente. Então, $E_{1} \times E_{2}$ é uma célula orientada pelo produto das orientações dadas de $E_{1}$ e de $E_{2}$. O ponto-base de $E_{1} \times E_{2}$ é o ponto $(*, *)$. O bordo $S=\left(E_{1} \times E_{2}\right)^{\cdot}=$ $E_{1} \times \dot{E}_{2} \cup \dot{E}_{1} \times E_{2}$ é então uma $(p+q+1)$-esfera orientada, e a aplicação $h:(S, *) \rightarrow(X, *)$ definida por

$$
h(x, y)= \begin{cases}f(x), & \left(x \in E_{1}, y \in \dot{E}_{2}\right), \\ g(y), & \left(x \in \dot{E}_{1}, y \in E_{2}\right),\end{cases}
$$

representa um elemento $[\alpha, \beta] \in \pi_{p+q+1}(X)$ que depende apenas das classes de homotopia $\alpha, \beta$ de $f, g$ respectivamente.

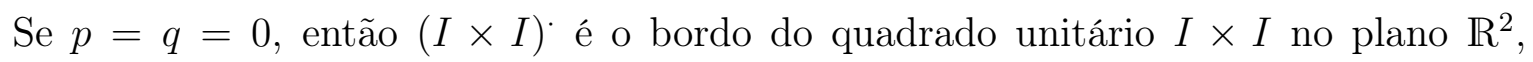
com orientação horária, e com a origem como ponto base. As aplicações $f, g$ são laços representando $\alpha, \beta$ e temos $[\alpha, \beta]=\alpha \beta \alpha^{-1} \beta^{-1} \in \pi_{1}(X)$. A notação $[\alpha, \beta]$ para o produto de Whitehead sugere à notação para comutador de dois elementos em um grupo.

Já em [10], Davis e Kirk apresentam a definição do produto de Whitehead da seguinte forma:

Definição 3.1.1. Seja a $: S^{k+n-1} \rightarrow S^{k} \vee S^{n}$ a aplicação de colagem da $(k+n)$-célula de $S^{k} \times S^{n}$. Dada $\alpha=[f] \in \pi_{k}(X)$ e $\beta=[g] \in \pi_{n}(X)$, defina o produto de Whitehead $[\alpha, \beta] \in \pi_{k+n-1}(X)$ como a (classe de homotopia da) composição

$$
S^{k+n-1} \underset{a}{\longrightarrow} S^{k} \vee S^{n} \underset{f \vee g}{\longrightarrow} X .
$$

Uma aplicação $h: S^{p} \times S^{q} \rightarrow X$ é dita ter o tipo $(\alpha, \beta)$ se, e somente se, $\left.h\right|_{S^{p} \times\{*\}}$ representa $\alpha \in \pi_{p}(X)$ e $\left.h\right|_{\{*\} \times S^{q}}$ representa $\beta \in \pi_{q}(X)$. Uma importante propriedade do produto de Whitehead é:

Teorema 3.1.2. Sejam $\alpha \in \pi_{p}(X), \beta \in \pi_{q}(x)$. Então existe uma aplicação de $S^{p} \times S^{q}$ em $X$ do tipo $(\alpha, \beta)$ se, e somente se, $[\alpha, \beta]=0$.

Corolário 3.1.3. Se X é um $H$-espaço, então $[\alpha, \beta]=0$ para todo $\alpha \in \pi_{p}(X), \beta \in \pi_{q}(X)$. 
Outra propriedade do produto de Whitehead é a 'bilinearidade', isto é, para todo $l, k \in \mathbb{Z}$ tem-se

$$
[l \alpha, k \beta]=l k[\alpha, \beta] .
$$

Temos, ainda, os seguintes resultados:

Teorema 3.1.4. Se $\alpha \in \pi_{p}(X), \beta \in \pi_{q}(X), \gamma \in \pi_{m}\left(S^{p}\right), \delta \in \pi_{n}\left(S^{q}\right)$, e se $[\alpha, \beta]=0$, então $[\alpha \circ \gamma, \beta \circ \delta]=0$.

Teorema 3.1.5. Sejam $\alpha \in \pi_{p+1}(X), \beta \in \pi_{q+1}(X), \gamma \in \pi_{m}\left(S^{p}\right), \delta \in \pi_{n}\left(S^{q}\right)$. Então

$$
[\alpha \circ \Sigma \gamma, \beta \circ \Sigma \delta]=[\alpha, \beta] \circ \Sigma(\gamma \wedge \delta) \text {. }
$$

Observemos que o produto smash $\gamma \wedge \delta \in \pi_{m+n}\left(S^{p+q}\right)$ pelo funtor definido em [47] pág. 480 .

Teorema 3.1.6. Se $\alpha \in \pi_{p}(X), \beta \in \pi_{q}(X)$, então $\Sigma[\alpha, \beta]=0$.

Também podemos encontrar em [47] pág. 536, informações sobre o produto de Whitehead que envolve apenas um elemento $\beta \in \pi_{r+1}(X)$.

Teorema 3.1.7. Seja $\beta \in \pi_{r+1}(X)$. Se r é par, $2[\beta, \beta]=0$ e todos os produtos de Whitehead em $\beta$, com 3 ou mais repetições, são zero. Se $r$ é impar, $3[[\beta, \beta], \beta]=0$ e todos os produtos em $\beta$ com 4 ou mais repetições, se anulam.

Em [47] pág. 549, temos que o núcleo do homomorfismo suspensão $\Sigma: \pi_{q}\left(S^{n}\right) \rightarrow$ $\pi_{q+1}\left(S^{n+1}\right)$ é gerado por todos os produtos de Whitehead $[\alpha, \beta] \operatorname{com} \alpha \in \pi_{r}\left(S^{n}\right), \beta \in$ $\pi_{s}\left(S^{n}\right)$ e $r+s=q+1$, se $q \leq 3 n-3$.

Para finalizarmos esta seção, podemos observar que em [1] foi demonstrado que $S^{p}$ tem estrutura de $H$-espaço se, e somente se, $p=1,3,7$. Nestes casos, a aplicação $f$ : $S^{p} \times S^{p} \rightarrow S^{p}$ é a multiplicação dos complexos, dos quatérnios e dos octônios de norma igual a 1 , respectivamente (ver também [8]).

Proposição 3.1.8. Se p é um número ímpar $p \neq 1,3,7$, ou seja, $S^{p}$ não é um $H$-espaço, então $[\gamma, \gamma]$ é um elemento de ordem 2 de $\pi_{2 p-1}\left(S^{p}\right)$, sendo $\gamma$ um gerador de $\pi_{p}\left(S^{p}\right) \cong \mathbb{Z}$.

\subsection{A seqüência exata $\Sigma H P$}

Denotemos por $\iota_{n} \in \pi_{n}\left(S^{n}\right)$ a classe de homotopia da aplicação identidade de $S^{n}$. Observe que, $\iota_{n} \circ \alpha=\alpha=\alpha \circ \iota_{p}$ para $\alpha \in \pi_{p}\left(S^{n}\right)$.

Em [20] podemos encontrar o conceito de produto reduzido de $S^{m}$ e alguns resultados necessários. Denotemos por $\left(S^{m}\right)_{\infty}$ o produto reduzido de $S^{m} \cdot\left(S^{m}\right)_{\infty}$ é um semi-grupo livre com o conjunto $S^{m}-e_{0}$ de geradores e a unidade $e_{0}$. Cada ponto de $\left(S^{m}\right)_{\infty}$ é representado por um produto $x_{1} \ldots x_{t}$ de $x_{1}, \ldots, x_{t} \in S^{m}$. Fixado um inteiro positivo $t$, $\left(S^{m}\right)_{t}$ denota o conjunto de todos os elementos $x_{1} \ldots x_{t}$. Então a topologia de $\left(S^{m}\right)_{t}$ é dada 
do produto $S^{m} \times \ldots \times S^{m}$ de $t$-vezes $S^{m}$ segundo a identificação: $S^{m} \times \ldots \times S^{m} \rightarrow\left(S^{m}\right)_{t}$ de $\left(x_{1}, \ldots, x_{t}\right) \mapsto x_{1} \ldots x_{t}$. $\left(S^{m}\right)_{t}-\left(S^{m}\right)_{t-1}$ é uma $t m$-célula aberta. Com a topologia fraca, $\left(S^{m}\right)_{\infty}$ é um $C W$-complexo e $\left(S^{m}\right)_{t}$ é seu tm-esqueleto. Identificamos $\left(S^{m}\right)_{1}$, com $S^{m}$ de maneira natural.

Considere uma aplicação $f:\left(S^{q}, e_{0}\right) \rightarrow\left(S^{m}, e_{0}\right)$, então um aplicação $(f)_{t}:\left(S^{q}\right)_{t} \rightarrow$ $\left(S^{m}\right)_{t}(t=1,2, \ldots, \infty)$ é dada pela fórmula $(f)_{t}\left(y_{1} \ldots y_{t}\right)=f\left(y_{1}\right) \ldots f\left(y_{t}\right)$. Naturalmente, $(f)_{s}=\left.(f)_{t}\right|_{\left(S^{q}\right)_{s}}$ para $s \leq t$.

A injeção canônica $i: S^{m} \rightarrow \Omega\left(S^{m+1}\right)$ é estendida a todo $\left(S^{m}\right)_{\infty}$ de tal modo que $x_{1} \ldots x_{t}$ é aplicado a um laço em $S^{m+1}$, o qual é representado por uma soma dos laços $i\left(x_{1}\right), \ldots, i\left(x_{t}\right)$. A aplicação resultante é injetiva, denotada por $i:\left(S^{m}\right)_{\infty} \rightarrow \Omega\left(S^{m+1}\right) \mathrm{e}$ chamada, também, por uma injeção canônica.

O seguinte diagrama é homotopicamente comutativo:

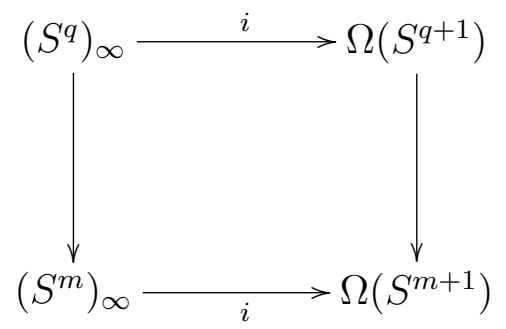

Um resultado sobre espaços de produtos reduzidos, é que a injeção canônica induz um isomorfismo de grupos de homotopia: $i_{q}: \pi_{q}\left(\left(S^{m}\right)_{\infty}\right) \rightarrow \pi_{q}\left(\Omega\left(S^{m+1}\right)\right)$ para todo $q$.

Temos o seguinte lema:

Lema 3.2.1. $i_{*}:\left[K,\left(S^{m}\right)_{\infty}\right]_{0} \rightarrow\left[K, \Omega\left(S^{m+1}\right)\right]_{0}$ é injetiva para um complexo celular finito arbitrário (ou, mais geral, um $C W$-complexo) $K$.

Definamos uma aplicação injetiva $\Omega_{1}$ por

$$
\Omega_{1}=i_{*}^{-1} \circ \bar{\Phi}:\left[\Sigma K, S^{m+1}\right]_{0} \rightarrow\left[K,\left(S^{m}\right)_{\infty}\right]_{0}
$$

lembrando que $\bar{\Phi}$ está definida no Teorema 1.1.4.

Em particular, temos o isomorfismo $\Omega_{1}: \pi_{k+1}\left(S^{m+1}\right) \approx \pi_{k}\left(\left(S^{m}\right)_{\infty}\right)$ para todo $k$.

Para a injeção $i^{\prime}: S^{m} \rightarrow\left(S^{m}\right)_{\infty}$, o seguinte diagrama é comutativo,

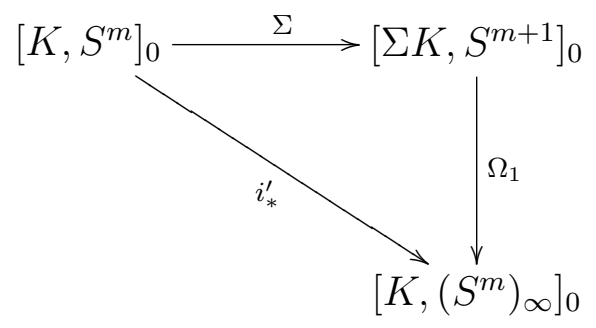

Consideremos, agora, a aplicação $h_{m}^{\prime}:\left(\left(S^{m}\right)_{2}, S^{m}\right) \rightarrow\left(S^{2 m}, e_{0}\right)$ dada por $h_{m}^{\prime}(x, y)=$ $\phi_{m, m}(x, y), x, y \in S^{m}$, em que $\phi_{m, m}$ é a aplicação que identifica $S^{m} \wedge S^{m} \operatorname{com} S^{2 m}$ definida em [46] 1.3 pág. 6. Seja 


$$
h_{m}:\left(\left(S^{m}\right)_{\infty}, S^{m}\right) \rightarrow\left(\left(S^{2 m}\right)_{\infty}, e_{0}\right)
$$

a extensão combinatorial ( veja [20]) de $h_{m}^{\prime}$. Podemos assim, definir o invariante de Hopf generalizado, por

$$
H=\Omega_{1}^{-1} \circ h_{m_{*}} \circ \Omega_{1}:\left[\Sigma K, S^{m+1}\right]_{0} \rightarrow\left[\Sigma K, S^{2 m+1}\right]_{0} .
$$

Em particular, temos homomorfismos $H: \pi_{i+1}\left(S^{m+1}\right) \rightarrow \pi_{i+1}\left(S^{2 m+1}\right)$.

Proposição 3.2.2. Sejam $K$ e $L$ complexos celulares finitos. Considere elementos $\alpha \in$ $\left[\Sigma K, S^{m+1}\right]_{0}, \beta \in[L, K]_{0}, \gamma \in \pi_{m}\left(S^{r}\right)$, então

$$
H(\alpha \circ \Sigma \beta)=H(\alpha) \circ \Sigma \beta
$$

$e$

$$
H(\Sigma \gamma \circ \alpha)=\Sigma(\gamma \wedge \gamma) \circ H(\alpha)
$$

Observemos que a discussão sobre $\left(S^{m}\right)_{\infty}$ e os resultados sobre o invariante de Hopf generalizado são verdadeiros para complexos celulares finitos.

Teorema 3.2.3. (James [21], Toda [44])Seja p um primo e $m>1$. Seja $h:\left(S^{m}\right)_{\infty} \rightarrow$ $\left(S^{p m}\right)_{\infty}$ uma aplicação que leva $\left(S^{m}\right)_{p-1}$ em $e_{0}$ e a célula e e homeomorfamente sobre $S^{p m}-e_{0}$. Se m é ímpar e $p=2$, então $h_{i}: \pi_{i}\left(\left(S^{m}\right)_{\infty}, S^{m}\right) \rightarrow \pi_{i}\left(\left(S^{2 m}\right)_{\infty}\right)$ são isomorfismos para todo $i$. Se $m$ é par, então $h_{i}: \pi_{i}\left(\left(S^{m}\right)_{\infty},\left(S^{m}\right)_{p-1}\right) \rightarrow \pi_{i}\left(\left(S^{p m}\right)_{\infty}\right)$ são isomorfismos para $i<(p+1) m-1$ e isomorfismos de componentes p-primárias para todo $i$.

A aplicação $h_{m}:\left(\left(S^{m}\right)_{\infty}, S^{m}\right) \rightarrow\left(\left(S^{2 m}\right)_{\infty}, e_{0}\right), m>1$, satisfaz a condição do teorema acima. Assim,

$$
h_{m_{i}}: \pi_{i}\left(\left(S^{m}\right)_{\infty}, S^{m}\right) \rightarrow \pi_{i}\left(\left(S^{2 m}\right)_{\infty}\right)
$$

são isomorfismos para todo $i$ se $m$ é ímpar e $i<3 m-1$ e isomorfismos das componentes 2-primária para todo $i$ se $m$ é par.

Agora, consideremos a seqüência exata do par $\left(\left(S^{m}\right)_{\infty}, S^{m}\right)$ :

$$
\cdots \longrightarrow \pi_{i}\left(S^{m}\right) \longrightarrow \pi_{i}\left(\left(S^{m}\right)_{\infty}\right) \longrightarrow \pi_{i}\left(\left(S^{m}\right)_{\infty}, S^{m}\right) \underset{\partial}{\longrightarrow} \pi_{i-1}\left(S^{m}\right) \longrightarrow \cdots
$$

Definamos o homomorfismo $P$ por

$$
P=\partial \circ h_{m_{i}}^{-1} \circ \Omega_{1}: \pi_{i+1}\left(S^{2 m+1}\right) \rightarrow \pi_{i-1}\left(S^{m}\right) / \partial\left(\mathrm{Nuch}_{m_{i}}\right),
$$

em que $\partial\left(\right.$ Nuch $\left._{m_{i}}\right)$ é trivial se $m$ é ímpar ou se $i<3 m-1$ e é finito e não tem 2 -torção se $m$ é par.

Também definimos um homomorfismo $P^{-1}$ por

$$
P^{-1}=\Omega_{1}^{-1} \circ h_{m_{*}} \circ \partial^{-1}: \pi_{i-1}\left(S^{m}\right) \rightarrow \pi_{i+1}\left(S^{2 m+1}\right) / H \pi_{i+1}\left(S^{m+1}\right),
$$

em que $H \pi_{i+1}\left(S^{m+1}\right)$ é o núcleo da aplicação $P$ acima. 
Da comutatividade de (3.2) e das definições de $H$ e $P$, temos uma seqüência exata

$$
\cdots \longrightarrow \pi_{i}\left(S^{m}\right) \underset{\Sigma}{\longrightarrow} \pi_{i+1}\left(S^{m+1}\right) \underset{H}{\longrightarrow} \pi_{i+1}\left(S^{2 m+1}\right) \underset{P}{\longrightarrow} \pi_{i-1}\left(S^{m}\right) \underset{\Sigma}{\longrightarrow} \cdots
$$

para $m$ ímpar ou $i<3 m-1$. Podemos considerar que a seqüência acima é uma seqüência exata das componentes 2-primárias no caso em que $m$ é par e $i \geq 3 m-1$.

Proposição 3.2.4. $P(\alpha) \circ \beta \subset P\left(\alpha \circ \Sigma^{2} \beta\right)$ para $\alpha \in \pi_{i+1}\left(S^{2 m+1}\right)$ e $\beta \in \pi_{j-1}\left(S^{i-1}\right)$. Em particular, $P\left(\Sigma^{2} \gamma\right)$ э $\pm\left[\iota_{m}, \iota_{m}\right] \circ \gamma$ em que [, ] indica o produto de Whitehead. Os simbolos $\subset$ e э podem ser trocados por $=$ se $m$ for impar, $j<3 m-1$ ou se os elementos na relação estão nas componentes 2-primária.

Temos que $H\left[\iota_{n}, \iota_{n}\right]= \pm 2 \iota_{2 n-1}$ para $n$ par. Então segue da proposição acima:

Proposição 3.2.5. $H\left(P\left(\iota_{2 n+1}\right)\right)= \pm 2 \iota_{2 n-1}$ para $n$ par.

Temos também por Serre [40] que $\pi_{i}\left(S^{n}\right)$ é finito exceto para os casos que $i=n$ e quando $n$ é par e $i=2 n-1$.

Denotamos a componente $p$-primária do grupo $\pi_{i}\left(S^{n}\right)$ por $\pi_{i}\left(S^{n} ; p\right), p$ primo.

Definamos um subgrupo $\pi_{i}^{n}$ de $\pi_{i}\left(S^{n}\right)$.

$$
\pi_{i}^{n}= \begin{cases}\pi_{n}\left(S^{n}\right), & \text { se } i=n \\ \Sigma^{-1}\left(\pi_{2 n}\left(S^{n+1} ; 2\right)\right), & \text { se } i=2 n-1, \\ \pi_{i}\left(S^{n} ; 2\right), & \text { se } i \neq n, 2 n-1\end{cases}
$$

O grupo $\pi_{2 n-1}^{n}$ é dado como segue.

Lema 3.2.6. Se $n$ é ímpar, então $\pi_{2 n-1}^{n}=\pi_{2 n-1}\left(S^{n} ; 2\right)$. Se $n$ é par e se $\left[\iota_{n-1}, \iota_{n-1}\right] \neq 0$, então $\pi_{2 n-1}^{n}$ é a soma direta de $\pi_{2 n-1}\left(S^{n} ; 2\right)$ e o grupo cíclico infinito $P\left(\pi_{2 n+1}\left(S^{2 n+1}\right)\right)$. Se $n$ é par e se $\left[\iota_{n-1}, \iota_{n-1}\right]=0$, então $\pi_{2 n-1}^{n}$ é a soma direta de $\pi_{2 n-1}\left(S^{n} ; 2\right)$ e um grupo cíclico infinito gerado por um elemento $\alpha$ tal que $H(\alpha)=\iota_{2 n-1}$ e $\Sigma \alpha \in \pi_{2 n}^{n+1}=\pi_{2 n}\left(S^{n+1} ; 2\right)$. Em todos os casos $\left[\iota_{n}, \iota_{n}\right]$ está contido em $\pi_{2 n-1}^{n}$.

A seguinte seqüência exata é a principal ferramente para calcular as componentes 2-primárias dos grupos de homotopia $\pi_{n+k}\left(S^{n}\right)$ :

Proposição 3.2.7. A seguinte seqüência é exata:

$$
\cdots \longrightarrow \pi_{i}^{n} \underset{\Sigma}{\longrightarrow} \pi_{i+1}^{n+1} \underset{H}{\longrightarrow} \pi_{i+1}^{2 n+1} \underset{P}{\longrightarrow} \pi_{i-1}^{n} \underset{\Sigma}{\longrightarrow} \pi_{i}^{n+1} \longrightarrow \cdots
$$

Lembramos que as provas dos resultados apresentados neste capítulo, junto com uma exposição mais completa, podem ser encontrados em Toda [46]. 
Sejam $p$ um primo e $\left(G_{k} ; p\right)$ a componente $p$-primária de grupo estável $G_{k}$. Pela exatidão da seqüência (3.5) temos que $\Sigma^{m-n}: \pi_{n+k}^{n} \rightarrow \pi_{m+k}^{m}, m \geq n$, e $\Sigma^{\infty}: \pi_{n+k}^{n} \rightarrow$ $\left(G_{k} ; 2\right)$ são isomorfismos para $n \geq k+2$.

Quando $p=2$ temos que se $n \geq 1, \alpha \in \pi_{k+n}\left(S^{m} ; 2\right), \gamma \in \pi_{i}\left(S^{j} ; 2\right)$. Então

$$
\alpha \circ \Sigma^{n} \pi_{j}\left(S^{k}\right)=\alpha \circ \Sigma^{n} \pi_{j}^{k} \subset \pi_{j+n}^{m}
$$

e

$$
\pi_{j}\left(S^{k}\right) \circ \gamma=\pi_{j}^{k} \circ \gamma \subset \pi_{i}^{k}
$$

Sabemos também que, para $n=2,4,8$, existe a aplicação de Hopf $h: S^{2 n-1} \rightarrow S^{n}$ de uma fibra $S^{n-1}$ e que

$$
\Sigma+h_{i}: \pi_{i-1}\left(S^{n-1}\right) \oplus \pi_{i}\left(S^{2 n-1}\right) \rightarrow \pi_{i}\left(S^{n}\right)
$$

é um isomorfismo para todo $i$ (ver [43]). O isomorfismo ainda continua se trocarmos $h$ por outra aplicação com invariante de Hopf igual a \pm 1 , pois os isomorfismos acima são induzidos pela aplicação $i \cdot \Omega h: S^{n-1} \times \Omega\left(S^{2 n-1}\right) \rightarrow \Omega\left(S^{n}\right)$, com ' . ' denotando a multiplicação-laço em $\Omega\left(S^{n}\right)$. Essa aplicação induz os isomorfismos dos grupos de cohomologia, de homologia e homotopia. E disso, temos:

Proposição 3.2.8. Seja $\alpha$ um elemento de $\pi_{2 n-1}^{n}$ tal que $H(\alpha)=\iota_{2 n-1}$ ou $-\iota_{2 n-1}$, então a correspondência $(\beta, \gamma) \mapsto \Sigma \beta+\alpha \circ \gamma$ produz isomorfismos de $\pi_{i-1}^{n-1} \oplus \pi_{i}^{2 n-1}$ sobre $\pi_{i}^{n}$ para todo $i$. Em particular $\Sigma: \pi_{i-1}^{n-1} \rightarrow \pi_{i}^{n}$ é um isomorfismo para todo $i$.

Na qual aplicamos:

Lema 3.2.9. Seja $n=2,4$ ou $8 . \Sigma: \pi_{i-1}\left(S^{n-1}\right) \rightarrow \pi_{i}\left(S^{n}\right)$ é um isomorfismo para todo $i$. $r \iota_{n} \circ \beta=r \beta$ para um inteiro arbitrário $r$ e para $\beta \in \pi_{i-1}\left(S^{n-1}\right)$.

Temos ainda que se $n$ é par e se $P\left(\iota_{2 n-1}\right)=\left[\iota_{n-1}, \iota_{n-1}\right] \neq 0$, então $\pi_{2 n-1}^{n}=\Sigma \pi_{2 n-2}^{n-1} \oplus$ $P \pi_{2 n+2}^{2 n+1} \approx \Sigma \pi_{2 n-2}^{n-1} \oplus \mathbb{Z}$ e $\pi_{2 n}^{n+1}=\Sigma^{2} \pi_{2 n-2}^{n-1} \approx \Sigma \pi_{2 n-2}^{n-1}$. Se $P\left(\iota_{2 n-1}\right)=\left[\iota_{n-1}, \iota_{n-1}\right]=0$, então $\Sigma^{2}: \pi_{2 n-2}^{n-1} \rightarrow \pi_{2 n}^{n+1}$ é um isomorfismo e $\pi_{2 n}^{n+1} / \Sigma^{2} \pi_{2 n-2}^{n-1} \approx \mathbb{Z} / 2$. Um elemento $\alpha$ de $\pi_{2 n-1}^{n}$ é aplicado em $\Sigma \pi_{2 n-2}^{n-1}$ por $\Sigma$ se, e somente se, $H(\alpha) \in 2 \pi_{2 n-1}^{2 n-1}$.

E temos também $\pi_{m}^{n} \circ \pi_{k}^{m} \subset \pi_{k}^{n}$.

\subsection{As componentes primárias de $\pi_{2+k}\left(S^{2}\right)$ e $\pi_{3+k}\left(S^{3}\right)$ com $1 \leq k \leq 19$}

Para um número primo $p$, a componente $p$-primária de um grupo abeliano é definida como o subgrupo consistindo dos elementos de ordem $p^{r}, r=1,2, \ldots$. Se temos um grupo de torção, então ele é a soma direta de suas componentes $p$-primárias para todo $p$. Assim, para calcular um certo grupo de torção é apenas necessário calcular todas as suas componentes $p$-primárias.

Iniciaremos com os cálculos para as componentes 2-primária de $\pi_{2+k}\left(S^{2}\right)$ e $\pi_{3+k}\left(S^{3}\right)$. À medida em que as componentes forem sendo calculadas, enunciaremos os resultados 
de Toda [46] para resultados mais gerais, ou seja, para dimensões maiores que 3. Algumas relações bastante usadas neste e no próximo capítulo estão listadas no Apêndice do Capítulo 4. Assim, para não nos tornarmos repetitivos, apenas indicamos que a relação se encontra no Apêndice. Já no final da seção, apresentaremos alguns resultados para as componentes $p$-primárias, com $p$ ímpar.

Temos, por Hu ([17] pág. 319 e 320), os seguintes resultados que nos mostram a importância de encontrar as componentes $p$-primárias dos grupos de homotopia de $S^{3}$.

Corolário 3.3.1. Se $n \geq 3$ é um inteiro impar, $p$ um número primo, e $m<n+4 p-6$, então as componetes p-primárias de $\pi_{m}\left(S^{n}\right)$ e $\pi_{m-n+3}\left(S^{3}\right)$ são isomorfas.

Corolário 3.3.2. Se $n \geq 3$ é um inteiro impar, $p$ um número primo, então a componente p-primária de $\pi_{m}\left(S^{n}\right)$ é 0 se $m<n+2 p-3$ e é $\mathbb{Z} / p$ se $m=n+2 p-3$.

Aqui, faremos os cálculos utilizando a seqüência $\Sigma H P$. Antes disso, definiremos composição secundária. Para uma tripla $(\alpha, \beta, \gamma)$ de elementos de $\pi_{k+n}\left(S^{m}\right) \times \pi_{j}\left(S^{k}\right) \times \pi_{i}\left(S^{j}\right)$, tendo como composições que se anulam $\alpha \circ \Sigma^{n} \beta=0$ e $\beta \circ \gamma=0$, a composição secundária

$$
\left\{\alpha, \Sigma^{n} \beta, \Sigma^{n} \gamma\right\}_{n} \subset \pi_{i+n+1}\left(S^{m}\right)
$$

está definida. Ela é a classe lateral do subgrupo $\alpha \circ \Sigma^{n} \pi_{i+1}\left(S^{k}\right)+\pi_{j+n+1}\left(S^{m}\right) \circ \Sigma_{n+1} \gamma$ de $\pi_{i+n+1}\left(S^{m}\right)$. A composição secundária é representada por uma composição $f \circ \Sigma^{n} g$ : $S^{i+n+1} \rightarrow \Sigma^{n} K \rightarrow S^{m}$ da $n$-ésima suspensão $\Sigma^{n} g$ de uma aplicação $g: S^{i+1} \rightarrow K$ e de uma aplicação $f: \Sigma^{n} K \rightarrow S^{n}$ tal que $K=S^{n} \sqcup e^{j+1}$ é um complexo celular tendo $\beta$ como a classe da aplicação de colagem de $e^{j+1}$. A restrição de $f$ sobre $\Sigma^{n} S^{k}=S^{n+k}$ representa $\alpha$ e $g$ aplica o hemisfério superior de $S^{i+1}$ em $K$ como a suspensão de um representante de $\gamma$ e o hemisfério inferior de $S^{i+1}$ em $S^{k}$.

Voltando agora ao cálculo dos grupos de homotopia, recordemos que $\pi_{i}^{1}=0$ para $i>1, \pi_{i}^{n}=0$ para $i<n$ e $\pi_{n}^{n} \approx \mathbb{Z}$. Disso, segue que $\pi_{2}^{1}=0$. Pela Proposição 3.2.8 temos que $H: \pi_{3}^{2} \rightarrow \pi_{3}^{3}$ é um isomorfismo. Seja $\eta_{2} \in \pi_{3}^{2}$ a classe tal que $H\left(\eta_{2}\right)=\iota_{3}$, então $\eta_{2}$ gera $\pi_{3}^{2} \approx \mathbb{Z}$. Denotemos por $\eta_{n}=\Sigma^{n-2} \eta_{2}$ para $n \geq 2$ e $\eta=\Sigma^{\infty} \eta_{2}$.

Pela Proposição 3.2.5, $H\left(P\left(\iota_{5}\right)\right)= \pm H\left[\iota_{2}, \iota_{2}\right]= \pm 2 \iota_{3}$. Assim, $P\left(\iota_{5}\right)= \pm 2 \eta_{3}$, já que $H: \pi_{3}^{2} \rightarrow \pi_{3}^{3}$ é um isomorfismo.

Considere a seqüência exata

$$
\pi_{5}^{5} \underset{P}{\longrightarrow} \pi_{3}^{2} \underset{\Sigma}{\longrightarrow} \pi_{4}^{3} \longrightarrow \pi_{5}^{4}=0
$$

Então $\Sigma$ é sobrejetiva e seu núcleo é gerado por $2 \eta_{2}$. Logo, $\pi_{4}^{3}=\left[\eta_{3}\right] \approx \mathbb{Z} / 2$.

Proposição 3.3.3. $\pi_{3}^{2}=\left[\eta_{2}\right] \approx \mathbb{Z}, \pi_{n+1}^{n}=\left[\eta_{n}\right] \approx \mathbb{Z} / 2$ para $n \geq 3$ e $\left(G_{1}, 2\right)=[\eta] \approx \mathbb{Z} / 2$. Temos as relações $H\left(\eta_{2}\right)=\iota_{3}$ e $P\left(\iota_{5}\right)= \pm 2 \eta_{3}$.

Denotemos $\eta_{n}^{2}=\eta_{n} \circ \eta_{n+1}$ e, do fato que a composição : $\alpha \rightarrow \eta_{2} \circ \alpha$ define um isomorfismo $\pi_{i}^{3} \approx \pi_{i}^{2}$ para $i \geq 3$, segue $\pi_{4}^{2}=\left[\eta_{2}^{2}\right] \approx \mathbb{Z} / 2$. 
Considere a seqüência exata de (3.5)

$$
\pi_{6}^{3} \underset{H}{\longrightarrow} \pi_{6}^{5} \underset{P}{\longrightarrow} \pi_{4}^{2} \underset{\Sigma}{\longrightarrow} \pi_{5}^{3} \underset{H}{\longrightarrow} \pi_{5}^{5} \underset{P}{\longrightarrow} \pi_{3}^{2} .
$$

Pela Proposição 3.3.3, $P: \pi_{5}^{5} \rightarrow \pi_{3}^{2}$ é um isomorfismo. Então $H\left(\pi_{5}^{3}\right)=0$ e $\Sigma$ é sobrejetiva. Pela relação (3) do Apêndice, e pela Proposição 3.3.3, $H: \pi_{6}^{3} \rightarrow \pi_{6}^{5}$ é sobrejetiva. Então $P\left(\pi_{6}^{5}\right)=0$ e $\Sigma$ é um isomorfismo. Assim, temos que $\Sigma: \pi_{4}^{2} \rightarrow \pi_{5}^{3}$ é um isomorfismo e temos que $\pi_{5}^{3}=\left[\eta_{3}^{2}\right] \approx \mathbb{Z} / 2$.

Proposição 3.3.4. $\pi_{n+2}^{n}=\left[\eta_{n}^{2}\right] \approx \mathbb{Z} / 2$ para $n \geq 2$. $\left(G_{2} ; 2\right)=\left[\eta^{2}\right] \approx \mathbb{Z} / 2$.

Dessa proposição e da relação (2) do Apêndice, temos que $\pi_{5}^{2}=\left[\eta_{2} \circ \eta_{3}^{2}\right]=\left[\eta_{2}^{3}\right] \approx \mathbb{Z} / 2$.

Um resultado bastante utilizado para determinarmos os geradores dos grupos é o seguinte ([46] pág. 40)

Lema 3.3.5. Assuma que $2 \alpha=0$ para um elemento $\alpha \in \pi_{i}\left(S^{3}\right)$, então para um elemento arbitrário $\beta \in\left\{\eta_{3}, e \iota_{4}, \Sigma \alpha\right\}_{1}$, as relações $H(\beta)=\Sigma^{2} \alpha$ e $2 \beta=\eta_{3} \circ \Sigma \alpha \circ \eta_{i+1}$ valem. $O$ elemento $\beta$ pertence a $\pi_{i+2}^{3} . P\left(\Sigma^{2} \alpha\right)=0$.

Temos que $\eta_{3}$ satisfaz o Lema acima e, para um elemento $\nu^{\prime}$ de $\left\{\eta_{3}, 2 \iota_{4}, \eta_{4}\right\}_{1}$, obtemos a relação (3) do Apêndice.

Considere a seqüência exata de (3.5)

$$
\pi_{7}^{3} \underset{H}{\longrightarrow} \pi_{7}^{5} \underset{P}{\longrightarrow} \pi_{5}^{2} \underset{\Sigma}{\longrightarrow} \pi_{6}^{3} \underset{H}{\longrightarrow} \pi_{6}^{5} \underset{P}{\longrightarrow} \pi_{4}^{2}
$$

Pela Proposição 3.2 .2 e pela relação (3) do Apêndice do Capítulo $4, H\left(\nu^{\prime} \circ \eta_{6}\right)=$ $\left(\nu^{\prime}\right) \circ \eta_{6}=\eta_{5} \circ \eta_{6}=\eta_{5}^{2}$. Então segue da Proposição 3.3.4, que $H: \pi_{7}^{3} \rightarrow \pi_{7}^{5}$ é sobrejetiva e assim $\Sigma$ é um isomorfismo. Com isso, $\eta_{3}^{3}=\Sigma \eta_{2}^{3}$ é de ordem 2. Segue da relação (3) do Apêndice do Capítulo 4 que $\nu^{\prime}$ é de ordem 4 . Como $\pi_{6}^{5}=\left[\eta_{5}\right] \approx \mathbb{Z} / 2, \nu^{\prime}$ é um gerador de $\pi_{6}^{3}$. Assim, $\pi_{6}^{3}=\left[\nu^{\prime}\right] \approx \mathbb{Z} / 4$.

Seja $\nu_{4}$ a classe da aplicação de Hopf $h_{4}: S^{7} \rightarrow S^{4}$. Então $\nu_{4}$ é um elemento de ordem infinita que gera o somando de ordem infinita $\pi_{7}=\mathbb{Z}\left[\nu_{4}\right] \oplus \mathbb{Z} / 12[\Sigma \xi]$ ([17] 16.3 pág. 329). Consideremos também $\nu_{n}=\Sigma^{n-4} \nu_{4}$.

Proposição 3.3.6. $\pi_{5}^{2}=\left[\eta_{2}^{3}\right] \approx \mathbb{Z} / 2$,

$\pi_{6}^{3}=\left[\nu^{\prime}\right] \approx \mathbb{Z} / 4$,

$\pi_{7}^{4}=\left[\nu_{4}\right] \oplus\left[\Sigma \nu^{\prime}\right] \approx \mathbb{Z} \oplus \mathbb{Z} / 4$

$\pi_{n+3}^{n}=\left[\nu_{n}\right] \approx \mathbb{Z} / 8$ para $n \geq 5$,

$\left(G_{3} ; 2\right)=[\nu] \approx \mathbb{Z} / 8$.

Dessa proposição e da relação (2) do Apêndice do Capítulo 4, temos $\pi_{6}^{2}=\left[\eta_{2} \circ \nu^{\prime}\right] \approx$ $\mathbb{Z} / 4$.

Da relação (8) do Apêndice do Capítulo 4, $\Sigma \pi_{6}^{2}=\left[\Sigma\left(\eta_{2} \circ \nu^{\prime}\right)\right]=0$, então temos uma seqüência exata $0 \longrightarrow \pi_{7}^{3} \longrightarrow \pi_{7}^{5}$ de (3.5). Pela relação (6) do Apêndice do Capítulo 4 e pela Proposição 3.3.4, temos que $H$ é um isomorfismo e $\pi_{7}^{3}=\left[\nu^{\prime} \circ \eta_{6}\right] \approx \mathbb{Z} / 2$. 
Proposição 3.3.7. $\pi_{6}^{2}=\left[\eta_{2} \circ \nu^{\prime}\right] \approx \mathbb{Z} / 4$,

$\pi_{7}^{3}=\left[\nu^{\prime} \circ \eta_{6}\right] \approx \mathbb{Z} / 2$,

$\pi_{8}^{4}=\left[\nu_{4} \circ \eta_{7}\right] \oplus\left[\Sigma \nu^{\prime} \circ \eta_{7}\right] \approx \mathbb{Z} / 2 \oplus \mathbb{Z} / 2$,

$\pi_{9}^{5}=\left[\nu_{5} \circ \eta_{8}\right] \approx \mathbb{Z} / 2$,

$\pi_{n+4}^{n}=0$ para $n \geq 6$,

$\left(G_{4} ; 2\right)=0$.

Dessa proposição e da relação (2) do Apêndice do Capítulo 4, temos $\pi_{7}^{2}=\left[\eta_{2} \circ \nu^{\prime} \circ \eta_{6}\right] \approx$ $\mathbb{Z} / 2$.

Pela relação (8) do Apêndice do Capítulo 4, $\Sigma \pi_{7}^{2}=\left[\sigma\left(\eta_{2} \circ \nu^{\prime}\right) \circ \eta_{7}\right]=0$. Segue da exatidão de (3.5) que a seqüência $0 \longrightarrow \pi_{8}^{3} \underset{H}{\longrightarrow} \pi_{8}^{5} \underset{P}{\longrightarrow} \pi_{6}^{2}$ é exata. Pelas relações (5) e (8) do Apêndice do Capítulo 4 e pela Proposição 3.3.6, temos que o núcleo de $P$ é gerado por $4 \nu_{5}=\eta_{5}^{3}$. Pela Proposição 3.2.2 e pela relação (3) do Apêndice do Capítulo 4,

$$
H\left(\nu^{\prime} \circ \eta_{6}^{2}\right)=H\left(\nu^{\prime}\right) \circ \eta_{6}^{2}=\eta_{5} \circ \eta_{6}^{2}=\eta_{5}^{3} .
$$

Segue da exatidão da seqüência acima $\pi_{8}^{3}=\left[\nu^{\prime} \circ \eta_{6}^{2}\right] \approx \mathbb{Z} / 2$.

Proposição 3.3.8. $\pi_{7}^{2}=\left[\eta_{2} \circ \nu^{\prime} \circ \eta_{6}\right] \approx \mathbb{Z} / 2$,

$\pi_{8}^{3}=\left[\nu^{\prime} \circ \eta_{6}^{2}\right] \approx \mathbb{Z} / 2$,

$\pi_{9}^{4}=\left[\nu_{4} \circ \eta_{7}^{2}\right] \oplus\left[\Sigma \nu^{\prime} \circ \eta_{7}^{2}\right] \approx \mathbb{Z} / 2 \oplus \mathbb{Z} / 2$,

$\pi_{10}^{5}=\left[\nu_{5} \circ \eta_{8}^{2}\right] \approx \mathbb{Z} / 2$

$\pi_{11}^{6}=\left[P\left(\iota_{13}\right)\right] \approx \mathbb{Z}$,

$\pi_{n+5}^{n}=\left(G_{5} ; 2\right)=0$ para $n \geq 7$. $\mathbb{Z} / 2$.

Dessa proposição e da relação (2) do Apêndice do Capítulo 4 temos $\pi_{8}^{2}=\left[\eta_{2} \circ \nu^{\prime} \circ \eta_{6}^{2}\right] \approx$

Pela relação (8) do Apêndice do Capítulo 4, $\Sigma \pi_{8}^{2}=\left[\Sigma\left(\eta_{2} \circ \nu^{\prime}\right) \circ \eta_{6}^{2}\right]=0$. Temos também $\Sigma \pi_{7}^{2}=0$ na Proposição 3.3.8. Segue da exatidão da seqüência (3.5) que a seqüência

$$
0 \longrightarrow \pi_{9}^{3} \underset{H}{\longrightarrow} \pi_{9}^{5} \underset{P}{\longrightarrow} \pi_{7}^{2} \longrightarrow 0
$$

é exata. Pela Proposições 3.3.7 e 3.3.8, temos que $P$ é um isomorfismo. Então segue que $\pi_{9}^{3}=0$.

Denotemos $\nu_{n}^{2}=\nu_{n} \circ \nu_{n+3}$ para $n \geq 4$ e $\nu^{2}=\nu \circ \nu$.

Proposição 3.3.9. $\pi_{8}^{2}=\left[\eta_{2} \circ \nu^{\prime} \circ \eta_{6}^{2}\right] \approx \mathbb{Z} / 2$,

$\pi_{9}^{3}=0$,

$\pi_{10}^{4}=\left[\nu_{4}^{2}\right] \approx \mathbb{Z} / 8$,

$\pi_{n+6}^{n}=\left[\nu_{n}^{2}\right] \approx \mathbb{Z} / 2$, para $n \geq 5$,

$\left(G_{6} ; 2\right)=\left[\nu^{2}\right] \approx \mathbb{Z} / 2$.

Dessa proposição e da relação (2) do Apêndice do Capítulo 4 temos que $\pi_{9}^{2}=0$.

Segue de (3.5) que a seqüência

$$
0=\pi_{9}^{2} \longrightarrow \pi_{10}^{3} \underset{H}{\longrightarrow} \pi_{10}^{5} \underset{P}{\longrightarrow} \pi_{8}^{2} \underset{\Sigma}{\longrightarrow} \pi_{9}^{3}=0
$$


é exata. Pelas Proposições 3.3 .8 e 3.3 .9 , vemos que $P$ é um isomorfismo. Segue que $\pi_{10}^{3}=0$.

O Lema 5.13 ([46] pág. 48), nos diz que $\sigma^{\prime \prime \prime}$ é o gerador de $\pi_{12}^{5} \approx \mathbb{Z} / 2$, e temos também:

Lema 3.3.10. Existem elementos $\sigma_{8} \in \pi_{15}^{8}, \sigma^{\prime} \in \pi_{14}^{7}$ e $\sigma^{\prime \prime} \in \pi_{13}^{6}$ tais que $H\left(\sigma_{8}\right)=$ $\iota_{15}, 2 \Sigma \sigma_{8}=\Sigma^{2} \sigma^{\prime \prime}, H\left(\sigma^{\prime}\right)=\eta_{13}, 2 \sigma^{\prime}=\Sigma \sigma^{\prime \prime}, H\left(\sigma^{\prime \prime}\right)=\eta_{11}^{2}, 2 \sigma^{\prime \prime}=\Sigma \sigma^{\prime \prime \prime}$.

E com isso,

Proposição 3.3.11. $\pi_{9}^{2}=\pi_{10}^{3}=\pi_{11}^{4}=0$,

$\pi_{12}^{5}=\left[\sigma^{\prime \prime \prime}\right] \approx \mathbb{Z} / 2$,

$\pi_{13}^{6}=\left[\sigma^{\prime \prime}\right] \approx \mathbb{Z} / 4$,

$\pi_{14}^{7}=\left[\sigma^{\prime}\right] \approx \mathbb{Z} / 8$,

$\pi_{15}^{8}=\left[\sigma_{8}\right] \oplus\left[\Sigma \sigma^{\prime}\right] \approx \mathbb{Z} \oplus \mathbb{Z} / 8$,

$\pi_{n+7}^{n}=\left[\sigma_{n}\right] \approx \mathbb{Z} / 16$, para $n \geq 9$,

$\left(G_{7} ; 2\right)=[\sigma] \approx \mathbb{Z} / 16$.

Consideremos agora os elementos:

- $\epsilon_{3} \in \pi_{11}^{3}$, com $\epsilon_{n}=\Sigma^{n-3} \epsilon_{3}$ para $n \geq 3$ e $\epsilon=\Sigma^{\infty} \epsilon_{3}$.

Temos o seguinte Lema:

Lema 3.3.12. $H\left(\epsilon_{3}\right)=\nu_{5}^{2}$ e $2 \epsilon_{n}=2 \epsilon=0, n \geq 3$.

- $\bar{\nu}_{6} \in \pi_{14}^{6} /\left[P\left(\nu_{13}\right)\right]$, com $\bar{\nu}_{n}=\Sigma^{n-6} \bar{\nu}_{6}$ para $n \geq 6$ e $\bar{\nu}=\Sigma^{\infty} \bar{\nu}_{6}$.

- $\mu_{3} \in \pi_{12}^{3}$, com $H\left(\mu_{3}\right)=\sigma^{\prime \prime \prime}, \mu_{n}=\Sigma^{n-3} \mu_{3}$ e $\mu=\Sigma^{\infty} \mu_{3}$.

- $\epsilon^{\prime} \in \pi_{13}^{3}$.

Temos o seguinte Lema:

Lema 3.3.13. $H\left(\epsilon^{\prime}\right)=\epsilon_{5}, 2 \epsilon^{\prime}=\eta_{3}^{2} \circ \epsilon_{5}$ e $\Sigma^{2} \epsilon^{\prime}$ é divisivel por 2 .

- $\zeta_{5} \in \pi_{16}^{5} /\left(\nu_{5} \circ \Sigma \pi_{15}^{7}\right), \operatorname{com} \zeta_{n}=\Sigma^{n-5} \zeta_{5}$ para $n \geq 5$ e $\zeta=\Sigma^{\infty} \zeta_{5}$.

Assim, temos primeiro que $\pi_{10}^{2}=0$ pela relação (2) do Apêndice do Capítulo 4 e pela Proposição 3.3.11. Na seqüência exata

$$
0=\pi_{10}^{2} \longrightarrow \pi_{11}^{3} \underset{H}{\longrightarrow} \pi_{11}^{5}
$$

de (3.5), $H\left(\epsilon_{3}\right)=\nu_{5}^{2}$ pelo Lema 3.3.12, e essa imagem gera $\pi_{11}^{5}$ pela Proposição 3.3.9. Segue então $\pi_{11}^{3}=\left[\epsilon_{3}\right] \approx \mathbb{Z} / 2$ e assim $\pi_{11}^{2}=\left[\eta_{2} \circ \epsilon_{3}\right] \approx \mathbb{Z} / 2$ pela relação (2) do Apêndice do Capítulo 4.

Consideremos agora a seqüência exata

$$
\pi_{13}^{5} \underset{P}{\longrightarrow} \pi_{11}^{2} \underset{\Sigma}{\longrightarrow} \pi_{12}^{3} \underset{H}{\longrightarrow} \pi_{12}^{5} \longrightarrow \pi_{10}^{2}=0
$$

de (3.5). Pelo Lema 3.3.5, $P \pi_{13}^{5}=\left[P\left(\epsilon_{5}\right)\right]=\left[P\left(\Sigma^{2} \epsilon_{3}\right)\right]=0$ já que $2 \epsilon_{3}=0$. Segue que $\Sigma$ é um isomorfismo. Pela Proposição 3.3 .11 e pela relação (10) do Apêndice do Capítulo 4, temos que $H$ é sobrejetiva e $\pi_{12}^{3}=\left[\mu_{3}\right] \oplus\left[\eta_{3} \circ \epsilon_{4}\right] \approx \mathbb{Z} / 2 \oplus \mathbb{Z} / 2$. 
Teorema 3.3.14. $\pi_{10}^{2}=0$,

$\pi_{n+8}^{n}=\left[\epsilon_{n}\right] \approx \mathbb{Z} / 2$, para $n=3,4,5$,

$\pi_{14}^{6}=\left[\bar{\nu}_{6}\right] \oplus\left[\epsilon_{6}\right] \approx \mathbb{Z} / 8 \oplus \mathbb{Z} / 2$,

$\pi_{15}^{7}=\left[\sigma^{\prime} \circ \eta_{14}\right] \oplus\left[\bar{\nu}_{7}\right] \oplus\left[\epsilon_{7}\right] \approx \mathbb{Z} / 2 \oplus \mathbb{Z} / 2 \oplus \mathbb{Z} / 2$,

$\pi_{16}^{8}=\left[\sigma_{8} \circ \eta_{15}\right] \oplus\left[\Sigma \sigma^{\prime} \circ \eta_{15}\right] \oplus\left[\bar{\nu}_{8}\right] \oplus\left[\epsilon_{8}\right] \approx \mathbb{Z} / 2 \oplus \mathbb{Z} / 2 \oplus \mathbb{Z} / 2 \oplus \mathbb{Z} / 2$,

$\pi_{17}^{9}=\left[\sigma_{9} \circ \eta_{16}\right] \oplus\left[\bar{\nu}_{9}\right] \oplus\left[\epsilon_{9}\right] \approx \mathbb{Z} / 2 \oplus \mathbb{Z} / 2 \oplus \mathbb{Z} / 2$,

$\pi_{n+8}^{n}=\left[\bar{\nu}_{n}\right] \oplus\left[\epsilon_{n}\right] \approx \mathbb{Z} / 2 \oplus \mathbb{Z} / 2$, para $n \geq 10$,

$\left(G_{8} ; 2\right)=[\bar{\nu}] \oplus[\epsilon] \approx \mathbb{Z} / 2 \oplus \mathbb{Z} / 2$.

Teorema 3.3.15. $\pi_{11}^{2}=\left[\eta_{2} \circ \epsilon_{3}\right] \approx \mathbb{Z} / 2$,

$\pi_{12}^{3}=\left[\mu_{3}\right] \oplus\left[\eta_{3} \circ \epsilon_{4}\right] \approx \mathbb{Z} / 2 \oplus \mathbb{Z} / 2$,

$\pi_{n+9}^{n}=\left[\nu_{n}^{3}\right] \oplus\left[\mu_{n}\right] \oplus\left[\eta_{n} \circ \epsilon_{n+1}\right] \approx \mathbb{Z} / 2 \oplus \mathbb{Z} / 2 \oplus \mathbb{Z} / 2$, para $n=4,5,6$,

$\pi_{16}^{7}=\left[\sigma^{\prime} \circ \eta_{14}^{2}\right] \oplus\left[\nu_{7}^{3}\right] \oplus\left[\mu_{7}\right] \oplus\left[\eta_{7} \circ \epsilon_{8}\right] \approx \mathbb{Z} / 2 \oplus \mathbb{Z} / 2 \oplus \mathbb{Z} / 2 \oplus \mathbb{Z} / 2$,

$\pi_{17}^{8}=\left[\sigma_{8} \circ \eta_{15}^{2}\right] \oplus\left[\Sigma \sigma^{\prime} \circ \eta_{15}^{2}\right] \oplus\left[\nu_{8}^{3}\right] \oplus\left[\mu_{8}\right] \oplus\left[\eta_{8} \circ \epsilon_{9}\right] \approx \mathbb{Z} / 2 \oplus \mathbb{Z} / 2 \oplus \mathbb{Z} / 2 \oplus \mathbb{Z} / 2 \oplus \mathbb{Z} / 2$,

$\pi_{18}^{9}=\left[\sigma_{9} \circ \eta_{16}^{2}\right] \oplus\left[\nu_{9}^{3}\right] \oplus\left[\mu_{9}\right] \oplus\left[\eta_{9} \circ \epsilon_{10}\right] \approx \mathbb{Z} / 2 \oplus \mathbb{Z} / 2 \oplus \mathbb{Z} / 2 \oplus \mathbb{Z} / 2$,

$\pi_{19}^{10}=\left[\Delta\left(\iota_{21}\right)\right] \oplus\left[\nu_{10}^{3}\right] \oplus\left[\mu_{10}\right] \oplus\left[\eta_{10} \circ \epsilon_{11}\right] \approx \mathbb{Z} \oplus \mathbb{Z} / 2 \oplus \mathbb{Z} / 2 \oplus \mathbb{Z} / 2$,

$\pi_{n+9}^{n}=\left[\nu_{n}^{3}\right] \oplus\left[\mu_{n}\right] \oplus\left[\eta_{n} \circ \epsilon_{n+1}\right] \approx \mathbb{Z} / 2 \oplus \mathbb{Z} / 2 \oplus \mathbb{Z} / 2$, para $n \geq 11$,

$\left(G_{9} ; 2\right)=\left[\nu^{3}\right] \oplus[\mu] \oplus[\eta \circ \epsilon] \approx \mathbb{Z} / 2 \oplus \mathbb{Z} / 2 \oplus \mathbb{Z} / 2$.

Aplicando o Lema 3.3.5 ao elemento $\mu_{3}$, temos um elemento $\mu^{\prime} \in \pi_{14}^{3}$, tal que $H\left(\mu^{\prime}\right)=$ $\Sigma^{2} \mu_{3}=\mu_{5}$ e $2 \mu^{\prime}=\eta_{3} \circ \mu_{4} \circ \eta_{13}$.

Desse último teorema e pela relação (2) do Apêndice do Capítulo 4, temos $\pi_{12}^{2}=$ $\left[\eta_{2} \circ \mu_{3}\right] \oplus\left[\eta_{2}^{2} \circ \epsilon_{4}\right] \approx \mathbb{Z} / 2 \oplus \mathbb{Z} / 2$.

A imagem de $H$ nas relações (12) e (13) do Apêndice do Capítulo 4, geram $\pi_{14}^{5}$, pelo Teorema 3.3.15. Assim, na seqüência exata

$$
\pi_{14}^{3} \underset{H}{\longrightarrow} \pi_{14}^{5} \underset{P}{\longrightarrow} \pi_{12}^{2} \underset{\Sigma}{\longrightarrow} \pi_{13}^{3} \underset{H}{\longrightarrow} \pi_{13}^{5}
$$

de (3.5), o homomorfismo $H: \pi_{14}^{3} \rightarrow \pi_{14}^{5}$ é sobrejetivo. Então temos que $\Sigma$ é um isomorfismo. Pelo Lema 3.3.13 e pelo Teorema 3.3.14 tem-se $H: \pi_{13}^{3} \rightarrow \pi_{13}^{5}$ sobrejetivo e o grupo $\pi_{13}^{3}$ é de ordem 8 e gerado por $\epsilon^{\prime}, \eta_{3}^{2} \circ \epsilon_{5}=2 \epsilon^{\prime}$ e $\eta_{3} \circ \mu_{4}$. Assim, $\pi_{13}^{3}=\left[\epsilon^{\prime}\right] \oplus\left[\eta_{3} \circ \mu_{4}\right] \approx \mathbb{Z} / 4 \oplus \mathbb{Z} / 2$.

Teorema 3.3.16. $\pi_{12}^{2}=\left[\eta_{2} \circ \epsilon_{4}\right] \oplus\left[\eta_{2} \circ \mu_{3}\right] \approx \mathbb{Z} / 2 \oplus \mathbb{Z} / 2$,

$\pi_{13}^{3}=\left[\epsilon^{\prime}\right] \oplus\left[\eta_{3} \circ \mu_{4}\right] \approx \mathbb{Z} / 4 \oplus \mathbb{Z} / 2$,

$\pi_{14}^{4}=\left[\nu_{4} \circ \sigma^{\prime}\right] \oplus\left[\Sigma \epsilon^{\prime}\right] \oplus\left[\eta_{4} \circ \mu_{5}\right] \approx \mathbb{Z} / 8 \oplus \mathbb{Z} / 4 \oplus \mathbb{Z} / 2$,

$\pi_{n+10}^{n}=\left[\nu_{n} \circ \sigma_{n+3}\right] \oplus\left[\eta_{n} \circ \mu_{n+1}\right] \approx \mathbb{Z} / 8 \oplus \mathbb{Z} / 2$, para $n=5,6,7$,

$\pi_{18}^{8}=\left[\sigma_{8} \circ \nu_{15}\right] \oplus\left[\nu_{8} \circ \sigma_{11}\right] \oplus\left[\eta_{8} \circ \mu_{9}\right] \approx \mathbb{Z} / 8 \oplus \mathbb{Z} / 8 \oplus \mathbb{Z} / 2$,

$\pi_{19}^{9}=\left[\sigma_{9} \circ \nu_{16}\right] \oplus\left[\eta_{9} \circ \mu_{10}\right] \approx \mathbb{Z} / 8 \oplus \mathbb{Z} / 2$

$\pi_{20}^{10}=\left[\sigma_{10} \circ \nu_{17}\right] \oplus\left[\eta_{10} \circ \mu_{11}\right] \approx \mathbb{Z} / 4 \oplus \mathbb{Z} / 2$,

$\pi_{21}^{11}=\left[\sigma_{11} \circ \nu_{18}\right] \oplus\left[\eta_{11} \circ \mu_{12}\right] \approx \mathbb{Z} / 2 \oplus \mathbb{Z} / 2$,

$\pi_{n+10}^{n}=\left[\eta_{n} \circ \mu_{n+1}\right] \approx \mathbb{Z} / 2$, para $n \geq 12$,

$\left(G_{10} ; 2\right)=[\eta \circ \mu] \approx \mathbb{Z} / 2$. 
Teorema 3.3.17. $\pi_{13}^{2}=\left[\eta_{2} \circ \epsilon^{\prime}\right] \oplus\left[\eta_{2}^{2} \circ \mu_{4}\right] \approx \mathbb{Z} / 4 \oplus \mathbb{Z} / 2$,

$\pi_{14}^{3}=\left[\mu^{\prime}\right] \oplus\left[\epsilon_{3} \circ \nu_{11}\right] \oplus\left[\nu^{\prime} \circ \epsilon_{6}\right] \approx \mathbb{Z} / 4 \oplus \mathbb{Z} / 2 \oplus \mathbb{Z} / 2$,

$\pi_{15}^{4}=\left[\nu_{4} \circ \sigma^{\prime} \circ \eta_{14}\right] \oplus\left[\nu_{4} \circ \bar{\nu}_{7}\right] \oplus\left[\nu_{4} \circ \epsilon_{7}\right] \oplus\left[\Sigma \mu^{\prime}\right] \oplus\left[\epsilon_{4} \circ \nu_{12}\right] \oplus\left[\Sigma \nu^{\prime} \circ \epsilon_{7}\right] \approx \mathbb{Z} / 2 \oplus \mathbb{Z} / 2 \oplus$ $\mathbb{Z} / 2 \oplus \mathbb{Z} / 4 \oplus \mathbb{Z} / 2 \oplus \mathbb{Z} / 2$,

$\pi_{16}^{5}=\left[\zeta_{5}\right] \oplus\left[\nu_{5} \circ \bar{\nu}_{8}\right] \oplus\left[\nu_{5} \circ \epsilon_{8}\right] \approx \mathbb{Z} / 8 \oplus \mathbb{Z} / 2 \oplus \mathbb{Z} / 2$,

$\pi_{17}^{6}=\left[\zeta_{6}\right] \oplus\left[\bar{\nu}_{6} \circ \nu_{14}\right] \approx \mathbb{Z} / 8 \oplus \mathbb{Z} / 4$,

$\pi_{n+11}^{n}=\left[\zeta_{n}\right] \oplus\left[\bar{\nu}_{n} \circ \nu_{n+8}\right] \approx \mathbb{Z} / 8 \oplus \mathbb{Z} / 2$, para $n=7,8,9$,

$\pi_{n+11}^{n}=\left[\zeta_{n}\right] \approx \mathbb{Z} / 8$, para $n=10,11$ e para $n \geq 13$,

$\pi_{23}^{12}=\left[P\left(\iota_{25}\right)\right] \oplus\left[\zeta_{12}\right] \approx \mathbb{Z} \oplus \mathbb{Z} / 8$,

$\left(G_{11} ; 2\right)=[\zeta] \approx \mathbb{Z} / 8$.

Desse último teorema, e pelas relações (2) e (16) do Apêndice do Capítulo 4, temos

$$
\begin{gathered}
\pi_{14}^{2}=\left[\eta_{2} \circ \mu^{\prime}\right] \oplus\left[\eta_{2} \circ \epsilon_{3} \circ \nu_{11}\right] \oplus\left[\eta_{2} \circ \nu^{\prime} \circ \epsilon_{6}\right] \\
=\left[\eta_{2} \circ \mu^{\prime}\right] \oplus\left[\eta_{2} \circ \nu^{\prime} \circ \bar{\nu}_{6}\right] \oplus\left[\eta_{2} \circ \nu^{\prime} \circ \epsilon_{6}\right] \approx \mathbb{Z} / 4 \oplus \mathbb{Z} / 2 \oplus \mathbb{Z} / 2 .
\end{gathered}
$$

Pela relação (8) do Apêndice do Capítulo 4, $\Sigma\left(\eta_{2} \circ \nu^{\prime} \circ \bar{\nu}_{6}\right)=\Sigma\left(\eta_{2} \circ \nu^{\prime} \circ \epsilon_{6}\right)=0$. Pela Proposição 3.2.4 e pelo Lema 3.3.5, $P\left(2 \zeta_{5}\right)=P\left( \pm \Sigma \mu^{\prime}\right)=P\left(\iota_{5}\right) \circ\left( \pm \mu^{\prime}\right)=2\left(\eta_{2} \circ \mu^{\prime}\right)$. Assim, $P\left(\zeta_{5}\right)= \pm\left(\eta_{2} \circ \mu^{\prime}\right) \bmod \left[\eta_{2} \circ \nu^{\prime} \circ \bar{\nu}_{6}\right]+\left[\eta_{2} \circ \nu^{\prime} \circ \epsilon_{6}\right]$ e $\Sigma\left(\eta_{2} \circ \mu^{\prime}\right)=0$. Então $\Sigma \pi_{14}^{2}=0$ e temos da exatidão de (3.5) que as seguintes seqüências são exatas.

$$
\begin{gathered}
0 \longrightarrow \pi_{15}^{3} \underset{H}{\longrightarrow} \pi_{15}^{5} \underset{P}{\longrightarrow} \pi_{13}^{2} \\
\pi_{17}^{5} \underset{P}{\longrightarrow} \pi_{15}^{2} \underset{\Sigma}{\longrightarrow} \pi_{16}^{3} \underset{H}{\longrightarrow} \pi_{16}^{5} \underset{P}{\longrightarrow} \pi_{14}^{2} \longrightarrow 0
\end{gathered}
$$

Pela relação (15) e pelo Teorema 3.3.17, a imagem de $\nu_{5} \circ \sigma_{8}$ segundo $P$ é um elemento de ordem 4 em $\pi_{13}^{2}$. Como $\pi_{15}^{5}=\left[\nu_{5} \circ \sigma_{8}\right] \oplus\left[\eta_{5} \circ \mu_{6}\right] \approx \mathbb{Z} / 8 \oplus \mathbb{Z} / 2$, pelo Teorema 3.3.16, o núcleo de $P: \pi_{15}^{5} \rightarrow \pi_{13}^{2}$ tem no máximo 4 elementos. Pela Proposição 3.2.2, e pelas relações (3) e (14) do Apêndice do Capítulo 4, temos

$$
H\left(\nu^{\prime} \circ \mu_{6}\right)=\eta_{5} \circ \mu_{6} \text { e } H\left(\nu^{\prime} \circ \eta_{6} \circ \epsilon_{7}\right)=\eta_{5}^{2} \epsilon_{7}=4\left(\nu_{5} \circ \sigma_{8}\right) .
$$

Esses elementos geram o núcleo do homomorfismo $P$ e segue da exatidão da primeira seqüência dada acima que

$$
\pi_{15}^{3}=\left[\nu^{\prime} \circ \mu_{6}\right] \oplus\left[\nu^{\prime} \circ \eta_{6} \circ \epsilon_{7}\right] \approx \mathbb{Z} / 2 \oplus \mathbb{Z} / 2
$$

Pela relação (2) do Apêndice do Capítulo 4,

$$
\pi_{15}^{2}=\left[\eta_{2} \circ \nu^{\prime} \circ \mu_{6}\right] \oplus\left[\eta_{2} \circ \nu^{\prime} \circ \eta_{6} \circ \epsilon_{7}\right] \approx \mathbb{Z} / 2 \oplus \mathbb{Z} / 2 .
$$

Temos, pela relação (8) do Apêndice do Capítulo 4 que

$$
\Sigma \pi_{15}^{2}=\left[\Sigma\left(\eta_{2} \circ \nu\right) \circ \mu_{7}\right] \oplus\left[\Sigma\left(\eta_{2} \circ \nu^{\prime} \circ \eta_{7} \circ \epsilon_{8}\right]=0 .\right.
$$

Segue da exatidão da segunda seqüência dada acima que $P: \pi_{17}^{5} \rightarrow \pi_{15}^{2}$ é sobrejetiva, e o grupo $\pi_{16}^{3}$ é isomorfo ao núcleo de $P: \pi_{16}^{5} \rightarrow \pi_{14}^{2}$ o qual é um homomorfismo sobrejetor. 
Como $\pi_{16}^{5} \approx \mathbb{Z} / 8 \oplus \mathbb{Z} / 2 \oplus \mathbb{Z} / 2$, pelo Teorema 3.3.17, então o núcleo de $P$ tem apenas 2 elementos 0 e $4 \zeta_{5}$. Pela Proposição 3.2.2, e pelas relações (3) e (17) do Apêndice do Capítulo 4,

$$
H\left(\nu^{\prime} \circ \eta_{6} \mu_{7}\right)=\eta_{5}^{2} \circ \mu_{7}=4 \zeta_{5}
$$

E assim,

$$
\pi_{16}^{3}=\left[\nu^{\prime} \circ \eta_{6} \circ \mu_{7}\right] \approx \mathbb{Z} / 2 .
$$

Teorema 3.3.18. $\pi_{14}^{2}=\left[\eta_{2} \circ \mu^{\prime}\right] \oplus\left[\eta_{2} \circ \nu^{\prime} \circ \bar{\nu}_{6}\right] \oplus\left[\eta_{2} \circ \nu^{\prime} \circ \epsilon_{6}\right] \approx \mathbb{Z} / 4 \oplus \mathbb{Z} / 2 \oplus \mathbb{Z} / 2$, $\pi_{15}^{3}=\left[\nu^{\prime} \circ \mu_{6}\right] \oplus\left[\nu^{\prime} \circ \eta_{6} \circ \epsilon_{7}\right] \approx \mathbb{Z} / 2 \oplus \mathbb{Z} / 2$,

$\pi_{16}^{4}=\left[\nu_{4} \circ \sigma^{\prime} \circ \eta_{14}^{2}\right] \oplus\left[\nu_{4}^{4}\right] \oplus\left[\nu_{4} \circ \mu_{7}\right] \oplus\left[\nu_{4} \circ \eta_{7} \circ \epsilon_{8}\right] \oplus\left[\sum \nu^{\prime} \circ \mu_{7}\right] \oplus\left[\Sigma \nu^{\prime} \circ \eta_{7} \circ \epsilon_{8}\right] \approx$ $\mathbb{Z} / 2 \oplus \mathbb{Z} / 2 \oplus \mathbb{Z} / 2 \oplus \mathbb{Z} / 2 \oplus \mathbb{Z} / 2 \oplus \mathbb{Z} / 2$,

$\pi_{17}^{5}=\left[\nu_{5}^{4}\right] \oplus\left[\nu_{5} \circ \mu_{8}\right] \oplus\left[\nu_{5} \circ \eta_{8} \circ \epsilon_{9}\right] \approx \mathbb{Z} / 2 \oplus \mathbb{Z} / 2 \oplus \mathbb{Z} / 2$,

$\pi_{18}^{6}=\left[P\left(\sigma_{13}\right)\right] \approx \mathbb{Z} / 16$,

$\pi_{n+12}^{n}=0$, para $n=7,8,9$,

$\pi_{22}^{10}=\left[P\left(\nu_{21}\right)\right] \approx \mathbb{Z} / 4$

$\pi_{23}^{11}=\left[\theta^{\prime}\right] \approx \mathbb{Z} / 2$,

$\pi_{24}^{12}=[\theta] \oplus\left[\Sigma \theta^{\prime}\right] \approx \mathbb{Z} / 2 \oplus \mathbb{Z} / 2$,

$\pi_{25}^{13}=[\Sigma \theta] \approx \mathbb{Z} / 2$,

$\pi_{n+12}^{n}=\left(G_{12} ; 2\right)=0$ para $n \geq 14$.

Teorema 3.3.19. $\pi_{15}^{2}=\left[\eta_{2} \circ \nu^{\prime} \circ \mu_{6}\right] \oplus\left[\eta_{2} \circ \nu^{\prime} \circ \eta_{6} \circ \epsilon_{7}\right] \approx \mathbb{Z} / 2 \oplus \mathbb{Z} / 2$,

$\pi_{16}^{3}=\left[\nu^{\prime} \circ \eta_{6} \circ \mu_{7}\right] \approx \mathbb{Z} / 2$,

$\pi_{17}^{4}=\left[\nu_{4}^{2} \circ \sigma_{8}\right] \oplus\left[\nu_{4} \circ \eta_{7} \circ \mu_{8}\right] \oplus\left[\Sigma \nu^{\prime} \circ \eta_{7} \circ \mu_{8}\right] \approx \mathbb{Z} / 8 \oplus \mathbb{Z} / 2 \oplus \mathbb{Z} / 2$,

$\pi_{18}^{5}=\left[\nu_{5} \circ \sigma_{8} \circ \nu_{5}\right] \oplus\left[\nu_{5} \circ \eta_{8} \circ \mu_{9}\right] \approx \mathbb{Z} / 2 \oplus \mathbb{Z} / 2$,

$\pi_{n+13}^{n}=\left[\nu_{n} \circ \sigma_{n+3} \circ \nu_{n+10}\right] \approx \mathbb{Z} / 2$, para $n=6,7$,

$\pi_{21}^{8}=\left[\sigma_{8} \circ \nu_{15}^{2}\right] \oplus\left[\nu_{8} \circ \sigma_{11} \circ \nu_{18}\right] \approx \mathbb{Z} / 2 \oplus \mathbb{Z} / 2$,

$\pi_{n+13}^{n}=\left[\sigma_{n} \circ \nu_{n+7}^{2}\right] \approx \mathbb{Z} / 2$, para $n=9,10$,

$\pi_{24}^{11}=\left[\theta^{\prime} \circ \eta_{23}\right] \oplus\left[\sigma_{11} \circ \nu_{18}^{2}\right] \approx \mathbb{Z} / 2 \oplus \mathbb{Z} / 2$,

$\pi_{25}^{12}=\left[\theta \circ \eta_{24}\right] \oplus\left[\Sigma \theta^{\prime} \circ \eta_{24}\right] \approx \mathbb{Z} / 2 \oplus \mathbb{Z} / 2$

$\pi_{26}^{13}=\left[\Sigma \theta \circ \eta_{25}\right] \approx \mathbb{Z} / 2$,

$\pi_{27}^{14}=\left[P\left(\iota_{29}\right)\right] \approx \mathbb{Z}$

$\pi_{n+13}^{n}=\left(G_{13} ; 2\right)=0$ para $n \geq 15$.

Os geradores $\theta$ e $\theta^{\prime}$ foram introduzidos no Lema 7.5 ([46] pág. 74).

Desse teorema e da relação (2) do Apêndice do Capítulo 4, temos $\pi_{16}^{2}=\left[\eta_{2} \circ \nu^{\prime} \circ \eta_{6} \circ \mu_{6}\right] \approx$ $\mathbb{Z} / 2$.

Pela Proposição 3.2.4 e pela relação (8) do Apêndice do Capítulo 4, temos:

$$
P\left(\nu_{5} \circ \eta_{8} \circ \mu_{9}\right)=\eta_{2} \circ \nu^{\prime} \circ \eta_{6} \circ \mu_{7} \text { e } \Sigma \pi_{16}^{2}=0 .
$$

Segue da exatidão da seqüência (3.5) que $H: \pi_{17}^{3} \rightarrow \pi_{17}^{5}$ aplica $\pi_{17}^{3}$ isomorficamente no núcleo de $P: \pi_{17}^{5} \rightarrow \pi_{15}^{2}$ a qual é sobrejetiva, como vimos na prova anterior. Pelos Teoremas 3.3.18 e 3.3.19, os grupos $\pi_{17}^{5}$ e $\pi_{15}^{2}$ têm 8 e 4 elementos, respectivamente. Assim, o núcleo de $P$ é isomorfo a $\mathbb{Z} / 2$. Pela Proposição 3.2.2 e Lema 3.3.12,

$$
H\left(\epsilon_{3} \circ \nu_{11}^{2}\right)=H\left(\epsilon_{3}\right) \circ \nu_{11}^{2}=\nu_{5}^{4},
$$


e $\nu_{5}^{4} \neq 0$ pelo Teorema 3.3.18. Conseqüentemente, obtemos

$$
\pi_{17}^{3}=\left[\epsilon_{3} \circ \nu_{11}^{2}\right] \approx \mathbb{Z} / 2 .
$$

Temos os elementos $\kappa_{7} \in \pi_{21}^{7}, \bar{\epsilon}_{3} \in \pi_{18}^{3}$, com $\kappa_{n}=\Sigma^{n-7} \kappa_{7}$ para $n \geq 7$ e $\kappa=\Sigma^{\infty} \kappa_{7}$, $\bar{\epsilon}_{n}=\Sigma^{n-3} \bar{\epsilon}_{3}$ para $n \geq 3$ e $\bar{\epsilon}=\Sigma^{\infty} \bar{\epsilon}_{3}$. Podemos enunciar o seguinte teorema

Teorema 3.3.20. $\pi_{16}^{2}=\left[\eta_{2} \circ \nu^{\prime} \circ \eta_{6} \circ \mu_{6}\right] \approx \mathbb{Z} / 2$,

$\pi_{17}^{3}=\left[\epsilon_{3} \circ \nu_{11}^{2}\right] \approx \mathbb{Z} / 2$,

$\pi_{18}^{4}=\left[\epsilon_{4} \circ \nu_{12}^{2}\right] \oplus\left[\nu_{4} \circ \zeta_{7}\right] \oplus\left[\nu_{4} \circ \bar{\nu}_{7} \circ \nu_{15}\right] \approx \mathbb{Z} / 2 \oplus \mathbb{Z} / 8 \oplus \mathbb{Z} / 2$,

$\pi_{19}^{5}=\left[\nu_{5} \circ \zeta_{8}\right] \oplus\left[\nu_{5} \circ \bar{\nu}_{8} \circ \nu_{16}\right] \approx \mathbb{Z} / 2 \oplus \mathbb{Z} / 2$,

$\pi_{20}^{6}=\left[\sigma^{\prime \prime} \circ \sigma_{13}\right] \oplus\left[\bar{\nu}_{6} \circ \nu_{14}^{2}\right] \approx \mathbb{Z} / 4 \oplus \mathbb{Z} / 2$,

$\pi_{21}^{7}=\left[\sigma^{\prime} \circ \sigma_{14}\right] \oplus\left[\kappa_{7}\right] \approx \mathbb{Z} / 8 \oplus \mathbb{Z} / 4$,

$\pi_{22}^{8}=\left[\sigma_{8}^{2}\right] \oplus\left[\Sigma \sigma^{\prime} \circ \sigma_{15}\right] \oplus\left[\kappa_{8}\right] \approx \mathbb{Z} / 16 \oplus \mathbb{Z} / 8 \oplus \mathbb{Z} / 4$,

$\pi_{23}^{9}=\left[\sigma_{9}^{2}\right] \oplus\left[\kappa_{9}\right] \approx \mathbb{Z} / 16 \oplus \mathbb{Z} / 4$,

$\pi_{n+14}^{n}=\left[\sigma_{n}^{2}\right] \oplus\left[\kappa_{n}\right] \approx \mathbb{Z} / 16 \oplus \mathbb{Z} / 2$, para $n=10,11,13$,

$\pi_{26}^{12}=\left[\sigma_{12}^{2}\right] \oplus\left[\kappa_{12}\right] \oplus\left[P\left(\nu_{25}\right)\right] \approx \mathbb{Z} / 16 \oplus \mathbb{Z} / 2 \oplus \mathbb{Z} / 4$,

$\pi_{28}^{14}=\left[\sigma_{14}^{2}\right] \oplus\left[\kappa_{14}\right] \approx \mathbb{Z} / 8 \oplus \mathbb{Z} / 2$,

$\pi_{29}^{15}=\left[\sigma_{15}^{2}\right] \oplus\left[\kappa_{15}\right] \approx \mathbb{Z} / 4 \oplus \mathbb{Z} / 2$,

$\pi_{n+14}^{n}=\left[\sigma_{n}^{2}\right] \oplus\left[\kappa_{n}\right] \approx \mathbb{Z} / 2 \oplus \mathbb{Z} / 2$, para $n \geq 16$,

$\left(G_{14} ; 2\right)=\left[\sigma^{2}\right] \oplus[\kappa] \approx \mathbb{Z} / 2 \oplus \mathbb{Z} / 2$ onde $\sigma_{n}^{2}=\sigma_{n} \circ \sigma_{n+7}$ e $\sigma^{2}=\sigma \circ \sigma$.

Desse Teorema e da relação (2) do Apêndice do Capítulo 4,

$$
\pi_{17}^{2}=\left[\eta_{2} \circ \epsilon_{3} \circ \nu_{11}^{2}\right] \approx \mathbb{Z} / 2 .
$$

Das relações (8) e (16) do Apêndice do Capítulo 4,

$$
\Sigma\left(\eta_{2} \circ \epsilon_{3} \circ \nu_{11}^{2}\right)=\Sigma\left(\eta_{2} \circ \nu^{\prime} \circ \bar{\nu}_{6} \circ \nu_{14}\right)=0 .
$$

Assim, $\Sigma \pi_{17}^{2}=0$. Segue da exatidão da seqüência (3.5) que as seguintes seqüências são exatas.

$$
\begin{gathered}
\pi_{19}^{5} \underset{P}{\longrightarrow} \pi_{17}^{2} \longrightarrow 0, \\
0 \longrightarrow \pi_{18}^{3} \underset{H}{\longrightarrow} \pi_{18}^{5} \underset{P}{\longrightarrow} \pi_{16}^{2} .
\end{gathered}
$$

Da prova anterior, temos que $P\left(\nu_{5} \circ \eta_{8} \circ \mu_{9}\right) \neq 0$. Como $\pi_{18}^{5}=\left[\nu_{5} \circ \eta_{8} \circ \mu_{9}\right] \oplus\left[\nu_{5} \circ \sigma_{8} \circ \nu_{15}\right] \approx$ $\mathbb{Z} / 2 \oplus \mathbb{Z} / 2$, pelo Teorema 3.3.19, então a imagem $H \pi_{18}^{3}$ tem no máximo 2 elementos. Pela relação (18) do Apêndice, $H\left(\bar{\epsilon}_{3}\right) \neq 0$. Temos assim,

$$
\pi_{18}^{3}=\left[\bar{\epsilon}_{3}\right] \approx \mathbb{Z} / 2
$$

Temos elementos $\rho^{I V} \in \pi_{20}^{5}, \rho^{\prime \prime \prime} \in \pi_{21}^{6}, \quad \rho^{\prime \prime} \in \pi_{22}^{7}$ e $\rho^{\prime} \in \pi_{24}^{9}$ tais que

$$
\begin{gathered}
H\left(\rho^{I V}\right)=\eta_{9}^{2} \circ \mu_{11}=4 \zeta_{9}, H\left(\rho^{\prime \prime \prime}\right)=\eta_{11} \circ \mu_{12} e \\
H\left(\rho^{\prime \prime}\right) \equiv \mu_{13} \bmod \left[\nu_{13}^{3}\right]+\left[\eta_{13} \circ \epsilon_{14}\right] \text { e } H\left(\rho^{\prime}\right)=8 \sigma_{17} .
\end{gathered}
$$


Teorema 3.3.21. $\pi_{17}^{2}=\left[\eta_{2} \circ \epsilon_{3} \circ \nu_{11}^{2}\right] \approx \mathbb{Z} / 2$,

$\pi_{n+15}^{n}=\left[\bar{\epsilon}_{n}\right] \approx \mathbb{Z} / 2$ para $n=3,4$,

$\pi_{20}^{5}=\left[\rho^{I V}\right] \oplus\left[\bar{\epsilon}_{5}\right] \approx \mathbb{Z} / 2 \oplus \mathbb{Z} / 2$,

$\pi_{21}^{6}=\left[\rho^{\prime \prime \prime}\right] \oplus\left[\bar{\epsilon}_{6}\right] \approx \mathbb{Z} / 4 \oplus \mathbb{Z} / 2$

$\pi_{22}^{7}=\left[\rho^{\prime \prime}\right] \oplus\left[\sigma^{\prime} \circ \bar{\nu}_{14}\right] \oplus\left[\sigma^{\prime} \circ \epsilon_{14}\right] \oplus\left[\bar{\epsilon}_{7}\right] \approx \mathbb{Z} / 8 \oplus \mathbb{Z} / 2 \oplus \mathbb{Z} / 2 \oplus \mathbb{Z} / 2$,

$\pi_{23}^{8}=\left[\sigma_{8} \circ \bar{\nu}_{15}\right] \oplus\left[\sigma_{8} \circ \epsilon_{15}\right] \oplus\left[\Sigma \rho^{\prime \prime}\right] \oplus\left[\Sigma \sigma^{\prime} \circ \bar{\nu}_{15}\right] \oplus\left[\Sigma \sigma^{\prime} \circ \epsilon_{15}\right] \oplus\left[\bar{\epsilon}_{8}\right] \approx \mathbb{Z} / 2 \oplus \mathbb{Z} / 2 \oplus \mathbb{Z} / 8 \oplus$ $\mathbb{Z} / 2 \oplus \mathbb{Z} / 2 \oplus \mathbb{Z} / 2$,

$\pi_{24}^{9}=\left[\rho^{\prime}\right] \oplus\left[\sigma_{9} \circ \bar{\nu}_{16}\right] \oplus\left[\sigma_{9} \circ \epsilon_{16}\right] \oplus\left[\bar{\epsilon}_{9}\right] \approx \mathbb{Z} / 16 \oplus \mathbb{Z} / 2 \oplus \mathbb{Z} / 2 \oplus \mathbb{Z} / 2$,

$\pi_{25}^{10}=\left[\Sigma \rho^{\prime}\right] \oplus\left[\sigma_{10} \circ \bar{\nu}_{17}\right] \oplus\left[\bar{\epsilon}_{10}\right] \approx \mathbb{Z} / 16 \oplus \mathbb{Z} / 2 \oplus \mathbb{Z} / 2$

$\pi_{26}^{11}=\left[\Sigma^{2} \rho^{\prime}\right] \oplus\left[\bar{\epsilon}_{11}\right] \approx \mathbb{Z} / 16 \oplus \mathbb{Z} / 2$,

$\pi_{27}^{12}=\left[\Sigma^{3} \rho^{\prime}\right] \oplus\left[\bar{\epsilon}_{12}\right] \approx \mathbb{Z} / 16 \oplus \mathbb{Z} / 2$,

Desse Teorema e da relação (2) do Apêndice do Capítulo 4,

$$
\pi_{18}^{2}=\left[\eta_{2} \circ \bar{\epsilon}_{3}\right] \approx \mathbb{Z} / 2
$$

Pela relação (20) do Apêndice do Capítulo 4, $P\left(\rho^{I V}\right)=P H\left(\bar{\mu}_{3}\right)=0$. Pelo Lema 3.3.5, $P\left(\bar{\epsilon}_{5}\right)=P\left(\Sigma^{2} \bar{\epsilon}_{3}\right)=0$. Segue de $(3.5)$, que a seguinte seqüência

$$
0 \longrightarrow \pi_{18}^{2} \underset{\Sigma}{\longrightarrow} \pi_{19}^{3} \underset{H}{\longrightarrow} \pi_{19}^{5} \underset{P}{\longrightarrow} \pi_{17}^{2}
$$

é exata. O último homomorfismo $P$ é sobrejetivo pela prova anterior. Como $\pi_{19}^{5} \approx$ $\mathbb{Z} / 2 \oplus \mathbb{Z} / 2$ e $\pi_{17}^{2}$ (Teoremas 3.3.20 e 3.3.21), então $H \pi_{19}^{3} \approx \mathbb{Z} / 2$. Pela Proposição 3.2.2, relação (10) do Apêndice do Capítulo 4, Lema 3.3.10 e Teorema 3.3.20,

$$
\Sigma H\left(\mu_{3} \circ \sigma_{12}\right)=\Sigma H\left(\mu_{3}\right) \circ \Sigma \sigma_{12}=\Sigma \sigma^{\prime \prime \prime} \circ \sigma_{13}=2 \sigma^{\prime \prime} \circ \sigma_{13} \neq 0 .
$$

Assim, $H\left(\mu_{3} \circ \sigma_{12}\right) \neq 0$. Obviamente $2\left(\mu_{3} \circ \sigma_{12}\right)=2 \mu_{3} \circ \sigma_{12}=0$. Conseqüentemente, temos da seqüência exata acima que

$$
\pi_{19}^{3}=\left[\mu_{3} \circ \sigma_{12}\right] \oplus\left[\eta_{3} \circ \bar{\epsilon}_{4}\right] \approx \mathbb{Z} / 2 \oplus \mathbb{Z} / 2
$$

Teorema 3.3.22. $\pi_{18}^{2}=\left[\eta_{2} \circ \bar{\epsilon}_{3}\right] \approx \mathbb{Z} / 2$,

$\pi_{n+16}^{n}=\left[\mu_{n} \circ \sigma_{n+9}\right] \oplus\left[\eta_{n} \circ \bar{\epsilon}_{n+1}\right] \approx \mathbb{Z} / 2 \oplus \mathbb{Z} / 2$ para $n=3,5$,

$\pi_{20}^{4}=\left[\nu_{4}^{2} \circ \sigma_{10} \circ \nu_{17}\right] \oplus\left[\mu_{4} \circ \sigma_{13}\right] \oplus\left[\eta_{4} \circ \bar{\epsilon}_{5}\right] \approx \mathbb{Z} / 2 \oplus \mathbb{Z} / 2 \oplus \mathbb{Z} / 2$,

$\pi_{22}^{6}=\left[\zeta^{\prime}\right] \oplus\left[\mu_{6} \circ \sigma_{15}\right] \oplus\left[\eta_{5} \circ \bar{\epsilon}_{7}\right] \approx \mathbb{Z} / 8 \oplus \mathbb{Z} / 2 \oplus \mathbb{Z} / 2$

$\pi_{23}^{7}=\left[\sigma^{\prime} \circ \mu_{14}\right] \oplus\left[\Sigma \zeta^{\prime}\right] \oplus\left[\mu_{7} \circ \sigma_{16}\right] \oplus\left[\eta_{7} \circ \bar{\epsilon}_{8}\right] \approx \mathbb{Z} / 2 \oplus \mathbb{Z} / 2 \oplus \mathbb{Z} / 2 \oplus \mathbb{Z} / 2$,

$\pi_{24}^{8}=\left[\sigma_{8} \circ \nu_{15}^{3}\right] \oplus\left[\sigma_{8} \circ \mu_{15}\right] \oplus\left[\sigma_{8} \circ \eta_{15} \circ \epsilon_{16}\right] \oplus\left[\Sigma \sigma^{\prime} \circ \mu_{15}\right] \oplus\left[\Sigma^{2} \zeta^{\prime}\right] \oplus\left[\mu_{8} \circ \sigma_{17}\right] \oplus\left[\eta_{8} \circ \bar{\epsilon}_{9}\right] \approx$ $\mathbb{Z} / 2 \oplus \mathbb{Z} / 2 \oplus \mathbb{Z} / 2 \oplus \mathbb{Z} / 2 \oplus \mathbb{Z} / 2 \oplus \mathbb{Z} / 2 \oplus \mathbb{Z} / 2$,

$\pi_{25}^{9}=\left[\sigma_{9} \circ \nu_{16}^{3}\right] \oplus\left[\sigma_{9} \circ \mu_{16}\right] \oplus\left[\sigma_{9} \circ \eta_{16} \circ \epsilon_{17}\right] \oplus\left[\mu_{9} \circ \sigma_{18}\right] \approx \mathbb{Z} / 2 \oplus \mathbb{Z} / 2 \oplus \mathbb{Z} / 2 \oplus \mathbb{Z} / 2$,

Temos ainda alguns elementos:

- $\bar{\mu}_{3} \in \pi_{20}^{3}$ e $\bar{\mu}_{n}=\Sigma^{n-3} \bar{\mu}_{3}$ para $n \geq 3$ e $\bar{\mu}=\Sigma^{\infty} \bar{\mu}_{3}$. 
- $\bar{\epsilon}^{\prime} \in \pi_{20}^{3}$.

- $\in \pi_{23}^{5}$, e $\bar{\zeta}_{n}=\Sigma^{n-5} \bar{\zeta}_{5}$ para $n \geq 5$ e $\bar{\zeta}=\Sigma^{\infty} \bar{\zeta}_{5}$.

- $\bar{\sigma}_{6} \in \pi_{25}^{6}$ e $\bar{\sigma}_{n}=\Sigma^{n-6} \bar{\sigma}_{6}$ para $n \geq 6$ e $\bar{\sigma}=\Sigma^{\infty} \bar{\sigma}_{6}$.

Desse último Teorema e da relação (2) do Apêndice do Capítulo 4,

$$
\pi_{19}^{2}=\left[\eta_{2} \circ \mu_{3} \circ \sigma_{12}\right] \oplus\left[\eta_{2}^{2} \circ \bar{\epsilon}_{4}\right] \approx \mathbb{Z} / 2 \oplus \mathbb{Z} / 2 .
$$

Considere a seqüência exata

$$
\pi_{21}^{5} \underset{P}{\longrightarrow} \pi_{19}^{2} \underset{\Sigma}{\longrightarrow} \pi_{20}^{3} \underset{H}{\longrightarrow} \pi_{20}^{5}
$$

de (3.5). Pelo Lema 3.3.5, temos que $P\left(\mu_{5} \circ \sigma_{14}\right)=\Delta\left(\eta_{5} \circ \bar{\epsilon}_{6}\right)=0$. Como $\mu_{5} \circ \sigma_{14}$ e $\eta_{5} \circ \bar{\epsilon}_{6}$ geram $\pi_{21}^{5}$ (Teorema 3.3.22), então $P$ é trivial e $\Sigma$ é um isomorfismo. Pelo Teorema 3.3.21, $\pi_{20}^{5} \approx \mathbb{Z} / 2 \oplus \mathbb{Z} / 2$. Assim $\pi_{20}^{3}$ tem no máximo 16 elementos. Pela relação (20) do Apêndice do Capítulo 4, $\bar{\mu}_{3}$ é um elemento de ordem 2 que não está contido em $\pi_{19}^{2}$, já que $H\left(\bar{\mu}_{3}\right) \neq 0$. Pela relação (21) do Apêndice do Capítulo 4, $\bar{\epsilon}^{\prime}$ é um elemento de ordem 4 que não está contido em $\Sigma \pi_{19}^{2} \approx \mathbb{Z} / 2 \oplus \mathbb{Z} / 2$. Então é uma conseqüência algébrica que

$$
\pi_{20}^{3}=\left[\bar{\epsilon}^{\prime}\right] \oplus\left[\bar{\mu}_{3}\right] \oplus\left[\eta_{3} \circ \mu_{4} \circ \sigma_{13}\right] \approx \mathbb{Z} / 4 \oplus \mathbb{Z} / 2 \oplus \mathbb{Z} / 2
$$

Teorema 3.3.23. $\pi_{19}^{2}=\left[\eta_{2} \circ \mu_{3} \circ \sigma_{12}\right] \oplus\left[\eta_{2}^{2} \circ \bar{\epsilon}_{4}\right] \approx \mathbb{Z} / 2 \oplus \mathbb{Z} / 2$,

$\pi_{20}^{3}=\left[\bar{\epsilon}^{\prime}\right] \oplus\left[\bar{\mu}_{3}\right] \oplus\left[\eta_{3} \circ \mu_{4} \circ \sigma_{13}\right] \approx \mathbb{Z} / 4 \oplus \mathbb{Z} / 2 \oplus \mathbb{Z} / 2$,

$\pi_{21}^{4}=\left[\nu_{4} \circ \sigma^{\prime} \circ \sigma_{14}\right] \oplus\left[\nu_{4} \circ \kappa_{7}\right] \oplus\left[\Sigma \bar{\epsilon}^{\prime}\right] \oplus\left[\eta_{4} \circ \mu_{5} \circ \sigma_{14}\right] \approx \mathbb{Z} / 8 \oplus \mathbb{Z} / 4 \oplus \mathbb{Z} / 4 \oplus \mathbb{Z} / 2 \oplus \mathbb{Z} / 2$,

$\pi_{22}^{5}=\left[\nu_{5} \circ \kappa_{8}\right] \oplus\left[\bar{\mu}_{5}\right] \oplus\left[\eta_{5} \circ \mu_{6} \circ \sigma_{15}\right] \approx \mathbb{Z} / 4 \oplus \mathbb{Z} / 2 \oplus \mathbb{Z} / 2$,

$\pi_{23}^{6}=[\Delta(\Sigma \theta)] \oplus\left[\nu_{6} \circ \kappa_{9}\right] \oplus\left[\bar{\mu}_{6}\right] \oplus\left[\eta_{6} \circ \mu_{7} \circ \sigma_{16}\right] \approx \mathbb{Z} / 2 \oplus \mathbb{Z} / 2 \oplus \mathbb{Z} / 2 \oplus \mathbb{Z} / 2$,

$\pi_{24}^{7}=\left[\sigma^{\prime} \circ \eta_{14} \circ \mu_{15}\right] \oplus\left[\nu_{7} \circ \kappa_{10}\right] \oplus\left[\bar{\mu}_{7}\right] \oplus\left[\eta_{7} \circ \mu_{8} \circ \sigma_{17}\right] \approx \mathbb{Z} / 2 \oplus \mathbb{Z} / 2 \oplus \mathbb{Z} / 2 \oplus \mathbb{Z} / 2$,

$\pi_{25}^{8}=\left[\sigma_{8} \circ \eta_{15} \circ \mu_{16}\right] \oplus\left[\Sigma \sigma^{\prime} \circ \eta_{15} \circ \mu_{16}\right] \oplus\left[\nu_{8} \circ \kappa_{11}\right] \oplus\left[\bar{\mu}_{8}\right] \oplus\left[\eta_{8} \circ \mu_{9} \circ \sigma_{18}\right] \approx \mathbb{Z} / 2 \oplus \mathbb{Z} / 2 \oplus$ $\mathbb{Z} / 2 \oplus \mathbb{Z} / 2 \oplus \mathbb{Z} / 2$,

$\pi_{26}^{9}=\left[\sigma_{9} \circ \eta_{16} \circ \mu_{17}\right] \oplus\left[\nu_{9} \circ \kappa_{12}\right] \oplus\left[\bar{\mu}_{9}\right] \oplus\left[\eta_{8} \circ \mu_{10} \circ \sigma_{19}\right] \approx \mathbb{Z} / 2 \oplus \mathbb{Z} / 2 \oplus \mathbb{Z} / 2 \oplus \mathbb{Z} / 2$,

Desse resultado e da relação (2) do Apêndice do Capítulo 4

$$
\pi_{20}^{2}=\left[\eta_{2} \circ \bar{\epsilon}^{\prime}\right] \oplus\left[\eta_{2} \circ \bar{\mu}_{3}\right] \oplus\left[\eta_{2}^{2} \circ \mu_{4} \circ \sigma_{13}\right] \approx \mathbb{Z} / 4 \oplus \mathbb{Z} / 2 \oplus \mathbb{Z} / 2 .
$$

Considere a seqüência exata

$$
\pi_{22}^{5} \underset{P}{\longrightarrow} \pi_{20}^{2} \underset{\Sigma}{\longrightarrow} \pi_{21}^{3} \underset{H}{\longrightarrow} \pi_{21}^{5}
$$

de (3.5). Pelo Lema 3.3.5, $P\left(\bar{\mu}_{5}\right)=\Delta\left(\eta_{5} \circ \mu_{6} \circ \sigma_{15}\right)=0$. Como $\Sigma^{2} \bar{\epsilon}^{\prime}=\Sigma^{2} \nu^{\prime} \circ \kappa_{8}=2 \iota_{5} \circ \nu_{5} \circ \kappa_{8}$, pela relação (21) do Apêndice do Capítulo 4, então segue da relação (8) do Apêndice do Capítulo 4 que $\Sigma\left(\eta_{2} \circ \bar{\epsilon}^{\prime}\right)=0$. Como $\pi_{22}^{5}=\left[\nu_{5} \circ \kappa_{8}\right] \oplus\left[\bar{\mu}_{5}\right] \oplus\left[\eta_{5} \circ \mu_{6} \circ \sigma_{15}\right] \approx \mathbb{Z} / 4 \oplus \mathbb{Z} / 2 \oplus \mathbb{Z} / 2$, pelo Teorema 3.3.23, segue que $\Delta\left(\nu_{5} \circ \kappa_{8}\right)= \pm\left(\eta_{2} \circ \bar{\epsilon}^{\prime}\right)$ e o núcleo de $P: \pi_{22}^{5} \rightarrow \pi_{20}^{2}$ é $\left[\bar{\mu}_{5}\right] \oplus\left[\eta_{5} \circ \mu_{6} \circ \sigma_{15}\right] \approx \mathbb{Z} / 2 \oplus \mathbb{Z} / 2$. Então,

$$
\pi_{20}^{2}=\left[\eta_{2} \circ \bar{\epsilon}^{\prime}\right] \oplus\left[\eta_{2} \circ \bar{\mu}_{3}\right] \oplus\left[\eta_{2}^{2} \circ \mu_{4} \circ \sigma_{13}\right] \approx \mathbb{Z} / 4 \oplus \mathbb{Z} / 2 \oplus \mathbb{Z} / 2,
$$




$$
\pi_{21}^{5}=\left[\mu_{5} \circ \sigma_{14}\right] \oplus\left[\eta_{5} \circ \bar{\epsilon}_{6}\right] \approx \mathbb{Z} / 2 \oplus \mathbb{Z} / 2
$$

pelo Teorema 3.3.22. Temos $H\left(\mu^{\prime} \circ \sigma_{14}\right)=\mu_{5} \circ \sigma_{14}$ pela Proposição 3.2 .2 e pela relação (12) do Apêndice do Capítulo 4 e $H\left(\nu^{\prime} \circ \bar{\epsilon}_{6}\right)=\eta_{5} \circ \bar{\epsilon}_{6}$ pela Proposição 3.2 .2 e pela relação (3) do Apêndice do Capítulo 4. Ainda, $2 \mu^{\prime} \circ \sigma_{14}=\eta_{3}^{2} \circ \mu_{5} \circ \sigma_{14}$ pela relação (12) do Apêndice do Capítulo 4 e $2\left(\nu^{\prime} \circ \bar{\epsilon}_{6}\right)=\nu^{\prime} \circ 2 \bar{\epsilon}_{6}=0$. Então segue da exatidão da seqüência acima que

$$
\pi_{21}^{3}=\left[\mu^{\prime} \circ \sigma_{14}\right] \oplus\left[\nu^{\prime} \circ \bar{\epsilon}_{6}\right] \oplus\left[\eta_{3} \circ \bar{\mu}_{4}\right] \approx \mathbb{Z} / 4 \oplus \mathbb{Z} / 2 \oplus \mathbb{Z} / 2 .
$$

Teorema 3.3.24. $\pi_{20}^{2}=\left[\eta_{2} \circ \bar{\epsilon}^{\prime}\right] \oplus\left[\eta_{2} \circ \bar{\mu}_{3}\right] \oplus\left[\eta_{2}^{2} \circ \mu_{4} \circ \sigma_{13}\right] \approx \mathbb{Z} / 4 \oplus \mathbb{Z} / 2 \oplus \mathbb{Z} / 2$, $\pi_{21}^{3}=\left[\mu^{\prime} \circ \sigma_{14}\right] \oplus\left[\nu^{\prime} \circ \bar{\epsilon}_{6}\right] \oplus\left[\eta_{3} \circ \bar{\mu}_{4}\right] \approx \mathbb{Z} / 4 \oplus \mathbb{Z} / 2 \oplus \mathbb{Z} / 2$,

$\pi_{22}^{4}=\left[\nu_{4} \circ \rho^{\prime \prime}\right] \oplus\left[\nu_{4} \circ \sigma^{\prime} \circ \bar{\nu}_{14}\right] \oplus\left[\nu_{4} \circ \sigma^{\prime} \circ \epsilon_{14}\right] \oplus\left[\nu_{4} \circ \bar{\epsilon}_{7}\right] \oplus\left[\Sigma \mu^{\prime} \circ \sigma_{15}\right] \oplus\left[\Sigma \nu^{\prime} \circ \bar{\epsilon}_{7}\right] \oplus\left[\nu_{4} \circ \bar{\mu}_{5}\right] \approx$ $\mathbb{Z} / 8 \oplus \mathbb{Z} / 2 \oplus \mathbb{Z} / 2 \oplus \mathbb{Z} / 2 \oplus \mathbb{Z} / 4 \oplus \mathbb{Z} / 2 \oplus \mathbb{Z} / 2$,

$\pi_{23}^{5}=\left[\zeta_{5} \circ \sigma_{16}\right] \oplus\left[\nu_{5} \circ \bar{\epsilon}_{8}\right] \oplus\left[\nu_{5} \circ \bar{\mu}_{6}\right] \approx \mathbb{Z} / 8 \oplus \mathbb{Z} / 2 \oplus \mathbb{Z} / 2$,

$\pi_{24}^{6}=\left[P(\Sigma \theta) \circ \eta_{23}\right] \oplus\left[\zeta_{6} \circ \sigma_{17}\right] \oplus\left[\eta_{6} \circ \bar{\mu}_{7}\right] \approx \mathbb{Z} / 2 \oplus \mathbb{Z} / 8 \oplus \mathbb{Z} / 2$,

$\pi_{25}^{7}=\left[\zeta_{7} \circ \sigma_{18}\right] \oplus\left[\eta_{7} \circ \bar{\mu}_{8}\right] \approx \mathbb{Z} / 8 \oplus \mathbb{Z} / 2$,

$\pi_{26}^{8}=\left[\sigma_{8} \circ \zeta_{15}\right] \oplus\left[\zeta_{8} \circ \sigma_{9}\right] \oplus\left[\eta_{8} \circ \bar{\mu}_{9}\right] \approx \mathbb{Z} / 8 \oplus \mathbb{Z} / 8 \oplus \mathbb{Z} / 2$,

$\pi_{27}^{9}=\left[\sigma_{9} \circ \zeta_{16}\right] \oplus\left[\eta_{9} \circ \bar{\mu}_{10}\right] \approx \mathbb{Z} / 8 \oplus \mathbb{Z} / 2$,

Desse Teorema e da relação (2) do Apêndice do Capítulo 4 temos

$$
\pi_{21}^{2}=\left[\eta_{2} \circ \mu^{\prime} \circ \sigma_{14}\right] \oplus\left[\eta_{2} \circ \nu^{\prime} \circ \bar{\epsilon}_{6}\right] \oplus\left[\eta_{2}^{2} \circ \bar{\mu}_{4}\right] \approx \mathbb{Z} / 4 \oplus \mathbb{Z} / 2 \oplus \mathbb{Z} / 2 .
$$

Considere a seqüência exata

$$
\pi_{23}^{5} \underset{P}{\longrightarrow} \pi_{21}^{2} \underset{\Sigma}{\longrightarrow} \pi_{22}^{3} \underset{H}{\longrightarrow} \pi_{22}^{5} \underset{P}{\longrightarrow} \pi_{20}^{2}
$$

de (3.5). Pelo Lema 3.3.5, $P\left(\eta_{5} \circ \bar{\mu}_{6}\right)=0$ e $2 P\left(\zeta_{5} \circ \sigma_{16}\right)=P\left( \pm \Sigma^{2} \bar{\mu}^{\prime} \circ \sigma_{16}\right)=2\left(\eta_{2} \circ \mu^{\prime} \circ \sigma_{14}\right)$. Segue que

$$
P\left(\zeta_{5} \circ \sigma_{16}\right)= \pm\left(\eta_{2} \circ \mu^{\prime} \circ \sigma_{15}\right) \bmod \left[\eta_{2} \circ \nu^{\prime} \circ \bar{\epsilon}_{6}\right]+\left[\eta_{2}^{2} \circ \bar{\mu}_{4}\right] .
$$

Pela Proposição 3.2.4 e pela relação (8) do Apêndice do Capítulo 4, $P\left(\nu_{5} \circ \bar{\epsilon}_{8}\right)=\left(\eta_{2} \circ \mu^{\prime} \circ \bar{\epsilon}_{6}\right)$. Como $\pi_{23}^{5}$ é gerado pelos elementos $\zeta_{5} \circ \sigma_{16}, \nu_{5} \circ \bar{\epsilon}_{8}$ e $\eta_{5} \circ \bar{\mu}_{6}$, pelo Teorema 3.3.24, então segue da exatidão da seqüência acima que

$$
\Sigma \pi_{21}^{2}=\left[\eta_{3}^{2} \circ \bar{\mu}_{5}\right] \approx \mathbb{Z} / 2 .
$$

Pela Proposição 3.2.2 e pela relação (3) do Apêndice do Capítulo 4,

$$
H\left(\nu^{\prime} \circ \mu_{6} \circ \sigma_{15}\right)=H\left(\nu^{\prime}\right) \circ \mu_{6} \circ \sigma_{15}=\eta_{5} \circ \mu_{6} \circ \sigma_{15} .
$$

Pela relação (22) do Apêndice do Capítulo 4,

$$
H\left(\bar{\mu}^{\prime}\right) \equiv \mu_{5} \bmod \Sigma^{3} \pi_{19}^{2} .
$$

Pelo Teorema 3.3.23 e por sua prova, temos que

$$
\Sigma^{3} \pi_{19}^{2}=\left[\eta_{5} \circ \mu_{6} \circ \sigma_{15}\right]+\left[4 \nu_{5} \circ \kappa_{8}\right]=\left[\eta_{5} \circ \mu_{6} \circ \sigma_{15}\right] .
$$


Então segue da exatidão da seqüência acima e da prova do Teorema anterior que as duas $H$-imagens geram $H \pi_{22}^{3} \approx \mathbb{Z} / 2 \oplus \mathbb{Z} / 2$. Pela relação (22) do Apêndice do Capítulo 4, $2 \bar{\mu}^{\prime}=\eta_{3}^{2} \circ \bar{\mu}_{5}$. Obviamente, $2 \nu^{\prime} \circ \mu_{6} \circ \sigma_{15}=\nu^{\prime} \circ 2 \mu_{6} \circ \sigma_{15}=0$. Logo, concluímos da seqüência exata acima que

$$
\pi_{22}^{3}=\left[\bar{\mu}^{\prime}\right] \oplus\left[\nu^{\prime} \circ \mu_{6} \circ \sigma_{15}\right] \approx \mathbb{Z} / 4 \oplus \mathbb{Z} / 2 .
$$

Teorema 3.3.25. $\pi_{21}^{2}=\left[\eta_{2} \circ \mu^{\prime} \circ \sigma_{14}\right] \oplus\left[\eta_{2} \circ \nu^{\prime} \circ \bar{\epsilon}_{6}\right] \oplus\left[\eta_{2}^{2} \circ \bar{\mu}_{4}\right] \approx \mathbb{Z} / 4 \oplus \mathbb{Z} / 2 \oplus \mathbb{Z} / 2$, $\pi_{22}^{3}=\left[\bar{\mu}^{\prime}\right] \oplus\left[\nu^{\prime} \circ \mu_{6} \circ \sigma_{15}\right] \approx \mathbb{Z} / 4 \oplus \mathbb{Z} / 2$,

$\pi_{23}^{4}=\left[\nu_{4} \circ \sigma^{\prime} \circ \mu_{14}\right] \oplus\left[\nu_{4} \circ \Sigma \zeta^{\prime}\right] \oplus\left[\nu_{4} \circ \mu_{7} \circ \sigma_{16}\right] \oplus\left[\nu_{4} \circ \eta_{7} \circ \bar{\epsilon}_{8}\right] \oplus\left[\Sigma \bar{\mu}^{\prime}\right] \oplus\left[\Sigma \nu^{\prime} \circ \mu_{7} \circ \sigma_{16}\right] \approx$ $\mathbb{Z} / 2 \oplus \mathbb{Z} / 2 \oplus \mathbb{Z} / 2 \oplus \mathbb{Z} / 2 \oplus \mathbb{Z} / 4 \oplus \mathbb{Z} / 2$,

$\pi_{24}^{5}=\left[\bar{\zeta}_{5}\right] \oplus\left[\nu_{5} \circ \mu_{8} \circ \sigma_{17}\right] \approx \mathbb{Z} / 8 \oplus \mathbb{Z} / 2$,

$\pi_{25}^{6}=\left[\bar{\zeta}_{6}\right] \oplus\left[\bar{\sigma}_{6}\right] \approx \mathbb{Z} / 8 \oplus \mathbb{Z} / 32$,

$\pi_{n+19}^{n}=\left[\bar{\zeta}_{n}\right] \oplus\left[\bar{\sigma}_{n}\right] \approx \mathbb{Z} / 8 \oplus \mathbb{Z} / 2$, para $n=7,8,9$.

Ainda seguindo Toda [46], vejamos as componentes $p$-primárias, com $p$ ímpar. Para isso, recordemos o isomorfismo de Serre [41]:

$$
\pi_{i-1}\left(S^{2 m-1} ; p\right)+\pi_{i}\left(S^{4 m-1} ; p\right) \approx \pi_{i}\left(S^{2 m} ; p\right)
$$

o qual é dado pela correspondência $(\alpha, \beta) \rightarrow \Sigma \alpha+\left[\iota_{2 m}, \iota_{2 m}\right] \circ \beta$. Assim, quando a esfera possuir dimensão par, o cálculo será feito por esse isomorfismo.

Agora, considerando a seqüência exata de homotopia associada ao par $\left(\Omega^{2}\left(S^{n+2}\right), S^{n}\right)$, temos a seguinte seqüência exata:

$$
\cdots \longrightarrow \pi_{i}\left(S^{n}\right) \underset{\Sigma^{2}}{\longrightarrow} \pi_{i+2}\left(S^{n+2}\right) \longrightarrow \pi_{i}\left(\Omega^{2}\left(S^{n+2}\right), S^{n}\right) \underset{\partial}{\longrightarrow} \pi_{i-1}\left(S^{n}\right) \longrightarrow \cdots
$$

Como os grupos $\pi_{i}\left(S^{n}\right)$ são finitos, exceto $\pi_{n}\left(S^{n}\right) \approx \mathbb{Z}$, e como $\Sigma^{2}: \pi_{n}\left(S^{n}\right) \rightarrow$ $\pi_{n+2}\left(S^{n+2}\right)$ são isomorfismos, então a seqüência exata induz a seguinte seqüência exata ( $n$ ímpar):

$$
\cdots \longrightarrow \pi_{i}\left(S^{n} ; p\right) \underset{\Sigma^{2}}{\longrightarrow} \pi_{i+2}\left(S^{n+2} ; p\right) \longrightarrow \pi_{i}\left(\Omega^{2}\left(S^{n+2}\right), S^{n} ; p\right) \underset{\partial}{\longrightarrow} \pi_{i-1}\left(S^{n} ; p\right) \longrightarrow \cdots
$$

A seguinte Proposição e o Corolário estão provados em [44].

Proposição 3.3.26. Sejam n impar e $p$ um número primo impar. Então existe uma seqüência exata

$$
\begin{aligned}
& \cdots \longrightarrow \pi_{i+2}\left(S^{p(n+1)+1} ; p\right) \underset{P}{\longrightarrow} \pi_{i}\left(S^{p(n+1)-1} ; p\right) \longrightarrow \\
& \longrightarrow \pi_{i-1}\left(\Omega^{2}\left(S^{n+2}\right), S^{n} ; p\right) \longrightarrow \pi_{i+1}\left(S^{p(n+1)+1} ; p\right) \longrightarrow \cdots
\end{aligned}
$$

para $i \geq p(n+1)-1$ tal que $\Delta \circ \Sigma^{2}=f_{p}$ para uma aplicação $f_{p}: S^{p(n+1)-1} \rightarrow S^{p(n+1)-1}$ de grau $p$. 
Corolário 3.3.27. Sejam n impar e p um número primo ímpar. Então temos um isomorfismo

$$
\pi_{i-1}\left(\Omega^{2}\left(S^{n+2}\right), S^{n} ; p\right) \approx \pi_{i}\left(S_{f}, S^{p(n+1)-1}\right)
$$

para $i \leq p^{2}(n+1)-2$, onde $S_{f}$ é o mapping cylinder de $f_{p}$.

Para o caso $n=1$, temos o seguinte:

Proposição 3.3.28. Seja p um número primo ímpar. Então existe uma seqüência exata

$$
\begin{gathered}
\cdots \longrightarrow \pi_{i+2}\left(S^{2 p+1} ; p\right) \underset{P}{\longrightarrow} \pi_{i}\left(S^{2 p-1} ; p\right) \underset{G}{\longrightarrow} \\
\underset{G}{\longrightarrow} \pi_{i+1}\left(S^{3} ; p\right) \underset{H}{\longrightarrow} \pi_{i+1}\left(S^{2 p+1} ; p\right) \longrightarrow \cdots
\end{gathered}
$$

para $i \geq 2 p-1$ tal que $P \circ \Sigma^{2}=f_{p_{*}} e G(\beta)=\alpha_{0} \circ \Sigma \beta$ para um gerador arbitrário $\alpha_{0}$ de $\pi_{2 p}\left(S^{3} ; p\right) \approx \mathbb{Z} / p$.

Recordemos os seguintes resultados para grupos estáveis. Do Teorema 4.15 de [45], temos:

$$
\left(G_{2 i(p-1)-1} ; p\right) \approx \mathbb{Z} / \text { p para } i=1,2, \ldots, p-1 \text { e }\left(G_{k} ; p\right)=0
$$

de outro modo para $k<2 p(p-1)-2$. Seja $\alpha_{1}$ um gerador de $\left(G_{2 p-3} ; p\right)$. Então as composições secundárias

$$
\alpha_{i+1}=\left\langle\alpha_{1}, p \iota, \alpha_{i}\right\rangle
$$

fornecem geradores de $\left(G_{2(i+1)(p-1)-1} ; p\right), i+1<p$, indutivamente.

Teorema 3.3.29. Seja $p$ um primo impar.

$\pi_{2 m+1+2 i(p-1)-2}\left(S^{2 m+1} ; p\right) \approx \mathbb{Z} /$ p para $1 \leq m<i$, e $i=2, \ldots, p-1$,

$\pi_{2 m+1+2 i(p-1)-1}\left(S^{2 m+1} ; p\right) \approx \mathbb{Z} / p$ para $m \geq 1$, e $i=1,2, \ldots, p-1$,

$\pi_{2 m+1+k}\left(S^{2 m+1} ; p\right)=0$ para $k<2 p(p-1)-2$.

Lema 3.3.30. Existe uma seqüência $\left\{\alpha_{i}(3 ; p)\right\}$ de elementos $\alpha_{i}(3 ; p) \in \pi_{2 i(p-1)+2}\left(S^{3} ; p\right)$ tal que $\alpha_{1}(3 ; p)$ é um gerador de $\pi_{2 p}\left(S^{3} ; p\right), p \alpha_{i}(3 ; p)=0$ e

$$
\alpha_{i+1}(3 ; p) \in\left\{\alpha_{i}(3 ; p), p \iota_{2 i(p-1)+2}, \Sigma^{2 i(p-1)-1} \alpha_{1}(3 ; p)\right\}_{1} .
$$

Denotemos

$$
\alpha_{i}(n ; p)=\Sigma^{n-3} \alpha_{i}(3 ; p) \text { para } n \geq 3 \text { e } \alpha_{i}=\Sigma^{\infty} \alpha_{i}(3 ; p) .
$$

E temos assim, a seguinte proposição:

Proposição 3.3.31. $\pi_{2 m+2 i(p-1)}\left(S^{2 m+1} ; p\right)=\left[\alpha_{i}(2 m+1 ; p)\right] \approx \mathbb{Z} / p$, para $1 \leq i<p e$ $m \geq 1$.

$\pi_{2 i(p-1)+1}\left(S^{2 m+1} ; p\right)=\left[\alpha_{i}(3) \circ \alpha_{i-1}(2 p ; p)\right] \approx \mathbb{Z} / p$, para $2 \leq i<p$.

No Apêndice do Capítulo 4 podemos encontrar uma tabela com os grupos de homotopia de $S^{3}$ calculados por Toda [46]. 


\section{Capítulo}

\section{Tipo de homotopia dos grupos de gauge dos $S^{3}$-fibrados sobre esferas}

Tendo estabelecido as ferramentas e os resultados básicos nos capítulos anteriores, podemos agora começar os cálculos do tipo de homotopia dos grupos de gauge dos $S^{3}$ fibrados sobre a esfera.

Consideremos então o conjunto $\operatorname{Princ}_{S^{3}}\left(S^{n}\right)$ dos $S^{3}$-fibrados principais $\xi$ sobre a esfera $S^{n}$, com $n \geq 1$. Tais fibrados são classificados pelas classes de homotopia de aplicações, ou seja, pelos conjuntos

$$
\left[S^{n}, B S^{3}\right]=\left[S^{n}, B S^{3}\right]_{0} \approx \pi_{n}\left(B S^{3}\right) \approx \pi_{n-1}\left(S^{3}\right)
$$

Por exemplo: $\operatorname{Princ}_{S^{3}}\left(S^{n}\right)=\{0\}$, o fibrado trivial, se $n \leq 3, \operatorname{Princ}_{S^{3}}\left(S^{4}\right) \approx \mathbb{Z}$, $\operatorname{Princ}_{S^{3}}\left(S^{5}\right) \approx \mathbb{Z} / 2, \operatorname{Princ}_{S^{3}}\left(S^{8}\right) \approx \mathbb{Z} / 12$. Devido a isso, assumiremos $n \geq 4$ daqui por diante.

Considere $f$ classificando $\xi_{f}$. Denotaremos por $\mathcal{G}_{f}$ o grupo de gauge do fibrado $\xi_{f}$. Então, temos as equivalências de homotopia (veja [38]):

$$
\begin{gathered}
\mathcal{G}_{f} \sim \Omega_{f} m\left(S^{n}, B S^{3} ; f\right), \\
B \mathcal{G}_{f} \sim m\left(S^{n}, B S^{3} ; f\right) .
\end{gathered}
$$

Consideremos agora a fibração avaliação

$$
e v_{f}: m\left(S^{n}, B S^{3} ; f\right) \rightarrow B S^{3},
$$

com $e v_{f}(g)=g\left(x_{0}\right)$, para qualquer $g \in m\left(S^{n}, B S^{3} ; f\right)$ e $x_{0}=(1,0, \ldots, 0) \in \mathbb{R}^{n+1}$ é o ponto-base de $S^{n}$. Pelo Lema 4.4.1 no Apêndice, temos as seqüências de fibrações e inclusões de fibras (a menos de homotopia)

$$
\begin{gathered}
\cdots \longrightarrow \mathcal{G}_{f} \underset{\Omega e v_{f}}{\longrightarrow} \Omega_{f} B S^{3} \sim S^{3} \longrightarrow m_{0}\left(S^{n}, B S^{3} ; f\right) \sim \Omega_{f}^{n-1} S^{3} \longrightarrow \\
\longrightarrow m\left(S^{n}, B S^{3} ; f\right) \underset{e v_{f}}{\longrightarrow} B S^{3} .
\end{gathered}
$$


Assim, conseguimos três fibrações

$$
\begin{gathered}
\mathcal{G}_{f} \longrightarrow S^{3} \longrightarrow \Omega_{f}^{n-1} S^{3} \\
S^{3} \longrightarrow \Omega_{f}^{n-1} S^{3} \longrightarrow m\left(S^{n}, B S^{3} ; f\right) \\
\Omega_{f}^{n-1} S^{3} \longrightarrow m\left(S^{n}, B S^{3} ; f\right) \underset{e v_{f}}{\longrightarrow} B S^{3} .
\end{gathered}
$$

O ponto principal é que, enquanto o tipo de homotopia das componentes conexas do espaço das aplicações livres depende da aplicação $f$ e, logo, do fibrado $\xi_{f}$, todas as componentes conexas do espaço das aplicações baseadas têm o mesmo tipo de homotopia [38], isto é, o tipo de $\Omega_{0}^{n-1} S^{3}$.

Em particular, isso significa que na primeira fibração podemos considerar $S^{3}$ e $\Omega_{0}^{n-1} S^{3}$ fixados, obtendo a fibração

$$
\mathcal{G}_{f} \longrightarrow S^{3} \longrightarrow \Omega_{0}^{n-1} S^{3}
$$

na qual $\mathcal{G}_{f}$ é a fibra (de homotopia) de uma aplicação $h: S^{3} \rightarrow \Omega_{0}^{n-1} S^{3}$. Assim, os possíveis diferentes tipos de $\mathcal{G}_{f}$ são dados pelas possíveis diferentes aplicações $h: S^{3} \rightarrow \Omega^{n-1} S^{3}$. Na verdade, se $h \sim g: S^{3} \rightarrow \Omega_{0}^{n-1} S^{3}$, então $F_{h} \sim F_{g}$, isto é, as duas fibras de homotopia são homotopicamente equivalentes ([47] ex. 6 pág. 44, Teorema 1.7 .30 pág. 42). Aqui, um certo cuidado é necessário. Na verdade estamos interessados nas equivalências de homotopia livre, ou seja, nos elementos

$$
[h] \in\left[S^{3}, \Omega_{0}^{n-1} S^{3}\right] \approx\left[S^{3}, \Omega_{0}^{n-1} S^{3}\right]_{0} / \pi_{1}\left(\Omega_{0}^{n-1} S^{3}\right) \approx \pi_{n+2}\left(S^{3}\right) / \pi_{n}\left(S^{3}\right) .
$$

Por ainda não conhecermos a ação de $\pi_{1}\left(\Omega_{0}^{n-1} S^{3}\right)$, iniciaremos por uma procura em distingüir as classes baseadas, afim de saber quem é $\pi_{n+2}\left(S^{3}\right)$. No que segue, faremos identificações que, de fato, darão as classes livres. Observando que as classes livres não podem ser mais que as classes baseadas, calcularemos primeiro as baseadas. Veremos, dessa forma, quantas das classes baseadas apresentam grupos de gauge diferentes. Mas isso será feito trabalhando diretamente sobre os grupos de homotopia dos grupos de gauge e, conseqüentemente, substituirá o problema de classes livres ou baseadas.

Assim, estamos interessados nas possíveis classes das aplicações $h: S^{3} \rightarrow \Omega_{0}^{n-1} S^{3}$, isto é, em $\pi_{n+2}\left(S^{3}\right)$. A saber, os diferentes tipos de homotopia dos grupos de gauge estão certamente contidos no conjunto $\pi_{n+2}\left(S^{3}\right)$ (de fato, se $F_{g} \nsim F_{h}$ então $g \nsim h$ ). Concluímos listando esses grupos juntamente com os grupos $\pi_{n-1}\left(S^{3}\right)$, cujas ordens indicam o número de diferentes fibrados sobre $S^{n}$. 


\begin{tabular}{|c|c|c|}
\hline & fibrados $\xi_{f}$ & grupos de gauge $\mathcal{G}_{f}$ \\
\hline$n$ & $\pi_{n-1}\left(S^{3}\right)$ & $\pi_{n+2}\left(S^{3}\right)$ \\
\hline 5 & $\mathbb{Z} / 2$ & $\mathbb{Z} / 2$ \\
\hline 6 & $\mathbb{Z} / 2$ & $\mathbb{Z} / 2$ \\
\hline 7 & $\mathbb{Z} / 12$ & $\mathbb{Z} / 3$ \\
\hline 8 & $\mathbb{Z} / 2$ & $\mathbb{Z} / 15$ \\
\hline 9 & $\mathbb{Z} / 2$ & $\mathbb{Z} / 2$ \\
\hline 10 & $\mathbb{Z} / 3$ & $(\mathbb{Z} / 2)^{2}$ \\
\hline 11 & $\mathbb{Z} / 15$ & $\mathbb{Z} / 2 \oplus \mathbb{Z} / 12$ \\
\hline 12 & $\mathbb{Z} / 2$ & $(\mathbb{Z} / 2)^{2} \oplus \mathbb{Z} / 84$ \\
\hline 13 & $(\mathbb{Z} / 2)^{2}$ & $(\mathbb{Z} / 2)^{2}$ \\
\hline 14 & $\mathbb{Z} / 2 \oplus \mathbb{Z} / 12$ & $\mathbb{Z} / 6$ \\
\hline 15 & $(\mathbb{Z} / 2)^{2} \oplus \mathbb{Z} / 84$ & $\mathbb{Z} / 30$ \\
\hline
\end{tabular}

\begin{tabular}{|c|c|c|}
\hline & fibrados $\xi_{f}$ & grupos de gauge $\mathcal{G}_{f}$ \\
\hline$n$ & $\pi_{n-1}\left(S^{3}\right)$ & $\pi_{n+2}\left(S^{3}\right)$ \\
\hline 16 & $(\mathbb{Z} / 2)^{2}$ & $\mathbb{Z} / 30$ \\
\hline 17 & $\mathbb{Z} / 6$ & $\mathbb{Z} / 2 \oplus \mathbb{Z} / 6$ \\
\hline 18 & $\mathbb{Z} / 30$ & $(\mathbb{Z} / 2)^{2} \oplus \mathbb{Z} / 12$ \\
\hline 19 & $\mathbb{Z} / 30$ & $(\mathbb{Z} / 2)^{2} \oplus \mathbb{Z} / 12$ \\
\hline 20 & $\mathbb{Z} / 2 \oplus \mathbb{Z} / 6$ & $\mathbb{Z} / 2 \oplus \mathbb{Z} / 132$ \\
\hline 21 & $(\mathbb{Z} / 2)^{2} \oplus \mathbb{Z} / 12$ & $(\mathbb{Z} / 2)^{2}$ \\
\hline 22 & $(\mathbb{Z} / 2)^{2} \oplus \mathbb{Z} / 12$ & $\mathbb{Z} / 2$ \\
\hline 23 & $\mathbb{Z} / 2 \oplus \mathbb{Z} / 132$ & $\mathbb{Z} / 210$ \\
\hline 24 & $(\mathbb{Z} / 2)^{2}$ & \\
\hline 25 & $\mathbb{Z} / 2$ & \\
\hline
\end{tabular}

Tabela 1.

\subsection{A imagem do homomorfismo $e v_{j}$}

Nesta seção, apresentaremos uma fórmula para calcular a imagem do homomorfismo ${ }^{1} e v_{j}$ induzido na homotopia pela aplicação avaliação

$$
e v_{f}: m\left(S^{n}, B S^{3} ; f\right) \rightarrow B S^{3} .
$$

Para isso, será melhor reduzir os cálculos ao grupo de homotopia de $S^{4}$ ao invés de $S^{3}$. De fato, embora exista o isomorfismo $\pi_{m}\left(S^{3}\right) \approx \pi_{m+1}\left(B S^{3}\right)$, este não se comporta bem com relação às operações de homotopia. Assim, usaremos o seguinte fato, onde $j: S^{4} \rightarrow B S^{3}$ é a inclusão do 1-esqueleto $\mathbb{H} P^{1}=S^{4}$ em $\mathbb{H} P^{\infty}=B S^{3}$ (veja [27]):

Lema 4.1.1. Qualquer aplicação de uma esfera em $B S^{3}$ se fatora (a menos de homotopia) pela inclusão $j: S^{4} \rightarrow B S^{3}$.

Demonstração: Isso significa que o homomorfismo $j_{n}: \pi_{n}\left(S^{4}\right) \rightarrow \pi_{n}\left(B S^{3}\right)$ tem uma inversa à direita $\psi$, a qual é definida como a composição do homomorfismo suspensão com o homomorfismo bordo, aparecendo na seqüência de homotopia associada a fibração universal para $B S^{3}$. Temos o diagrama (sempre assumindo que $n \geq 4$ ):

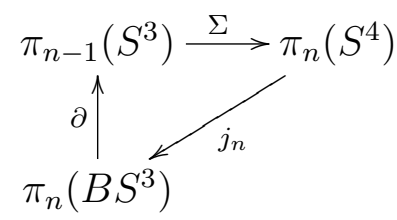

e $j_{n} \psi=1$, onde $\psi=\Sigma \partial$. Logo, $j_{n}$ é sobrejetiva e $\psi$ é injetiva.

\footnotetext{
${ }^{1}$ Omitiremos a indicação com relação à aplicação $f$ quando tratarmos do homomorfismo induzido na homotopia pela aplicação avaliação para não carregar demais a notação.
} 
Se $n=4, \Sigma$ é um isomorfismo, o que implica em $j_{4}$ ser um isomorfismo também e, conseqüentemente, $\pi_{4}\left(B S^{3}\right) \approx \mathbb{Z}\left[j_{4}\left(\iota_{4}\right)=\partial^{-1}\left(\iota_{3}\right)\right]$.

Lema 4.1.2. Para todo $k>1$, todo $n \geq 4 e[f] \in \pi_{n}\left(B S^{3}\right)$, temos a seguinte seqüência exata longa:

$$
\begin{gathered}
\cdots \longrightarrow \pi_{k+1}\left(B S^{3}\right) \underset{\partial_{k+1}}{\longrightarrow} \pi_{k+n}\left(B S^{3}\right) \longrightarrow \\
\longrightarrow \pi_{k-1}\left(\mathcal{G}_{f}\right) \underset{e v_{k}}{\longrightarrow} \pi_{k}\left(B S^{3}\right) \underset{\partial_{k}}{\longrightarrow} \pi_{k+n-1}\left(B S^{3}\right) \longrightarrow \cdots
\end{gathered}
$$

$e$

$$
\operatorname{Im} e v_{k}=\operatorname{ker} \partial_{k}=\left\{[u] \in \pi_{k}\left(B S^{3}\right) \mid[[u],[f]]=0 \in \pi_{n+k-1}\left(B S^{3}\right)\right\} .
$$

Demonstração: Considere a fibração avaliação introduzida no início deste capítulo,

$$
\Omega_{0}^{n-1} S^{3} \longrightarrow m\left(S^{n}, B S^{3} ; f\right) \underset{e v}{\longrightarrow} B S^{3}
$$

e a seqüência exata longa de homotopia associada

$$
\begin{gathered}
\cdots \longrightarrow \pi_{k+1}\left(m\left(S^{n}, B S^{3} ; f\right)\right) \underset{e v_{(k+1)}}{\longrightarrow} \pi_{k+1}\left(B S^{3}\right) \underset{\partial_{k+1}}{\longrightarrow} \\
\underset{\partial_{k+1}}{\longrightarrow} \pi_{k}\left(\Omega_{0}^{n-1} S^{3}\right) \longrightarrow \pi_{k}\left(m\left(S^{n}, B S^{3} ; f\right)\right) \underset{e v_{k}}{\longrightarrow} \pi_{k}\left(B S^{3}\right) \longrightarrow \cdots
\end{gathered}
$$

Recordando que $m\left(S^{n}, B S^{3} ; f\right) \sim B \mathcal{G}_{f}$, temos a seqüência exata longa

$$
\begin{gathered}
\cdots \longrightarrow \pi_{k+1}\left(B S^{3}\right) \underset{\partial_{k+1}}{\longrightarrow} \pi_{k+n-1}\left(S^{3}\right) \longrightarrow \\
\longrightarrow \pi_{k}\left(B \mathcal{G}_{f}\right) \underset{e v_{k}}{\longrightarrow} \pi_{k}\left(B S^{3}\right) \underset{\partial_{k}}{\longrightarrow} \pi_{k+n-2}\left(S^{3}\right) \longrightarrow \cdots
\end{gathered}
$$

e como $\pi_{k-1}\left(S^{3}\right) \approx \pi_{k}\left(B S^{3}\right)$ pelo Lema 4.4.1, temos a seqüência enunciada na tese, na qual o homomorfismo $\partial_{k}$ é a composição $\partial^{-1} \partial_{k}$, mas será denotado pela mesma notação de $\partial_{k}$, já que $\partial$ é um isomorfismo.

Agora, $e v_{k}$ é o homomorfismo induzido na homotopia pela aplicação:

$$
\Omega^{k} e v_{f}: m_{0}\left(S^{k}, m\left(S^{n}, B S^{3} ; f\right)\right) \rightarrow m_{0}\left(S^{k}, B S^{3}\right) .
$$

Note que

$$
\begin{gathered}
m_{0}(X, m(Y, Z ; f))=\left\{h: X \rightarrow m(Y, Z ; f): h\left(x_{0}\right)=f\right\}= \\
=\left\{a d(h): X \times Y \rightarrow Z: a d(h)\left(x_{0}, y\right)=f\right\}=\left\{g: X \times Y \rightarrow Z:\left.g\right|_{\left\{x_{0}\right\} \times Y}=f\right\} .
\end{gathered}
$$

Logo,

$$
m_{0}\left(S^{k}, m\left(S^{n}, B S^{3} ; f\right)\right)=\left\{g: S^{k} \times S^{n} \rightarrow B S^{3}:\left.g\right|_{\left\{x_{0}\right\} \times S^{n}}=f\right\}
$$

e

$$
\Omega^{k} e v_{f}: g \mapsto g\left(\cdot, y_{0}\right)
$$


(de fato, $\operatorname{ad}(h) \mapsto \operatorname{ad}(h)\left(\cdot, y_{0}\right)$ tal que $\left.h(x)(y) \mapsto h(x)\left(y_{0}\right)\right)$.

Assim, uma aplicação $u \in m_{0}\left(S^{k}, B S^{3}\right)$ está na imagem de $\Omega^{k} e v_{f}$ se, e somente se, existe uma aplicação $g: S^{k} \times S^{n} \rightarrow B S^{3}$ tal que $\left.g\right|_{\left\{x_{0}\right\} \times S^{n}}=f$ e $\left.g\right|_{S^{k} \times\left\{y_{0}\right\}}=u$. Como $S^{k} \times S^{n}$ é o push out do wedge com uma $(n+k)$-célula, temos a inclusão co-fibra $i$ : $S^{k} \vee S^{n}=\left\{x_{0}\right\} \times S^{n} \cup S^{k} \times\left\{y_{0}\right\} \rightarrow S^{k} \times S^{n}$ e $g$ existe se, e somente se, o seguinte diagrama comuta:

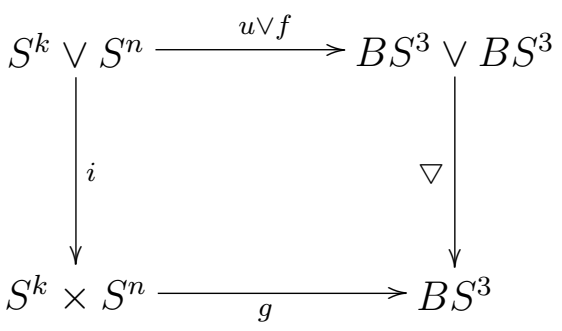

Mas isso significa que $g$ é uma extensão de $\nabla(u \vee f)$, e como $S^{k} \times S^{n}=\left(S^{k} \vee S^{n}\right) \sqcup_{a} e^{n+k}$ ([10] 10.11 pág. 319), sendo $a$ a aplicação de colagem da borda de célula $a: S^{n+k-1} \rightarrow$ $S^{k} \vee S^{n}$, temos o diagrama push out

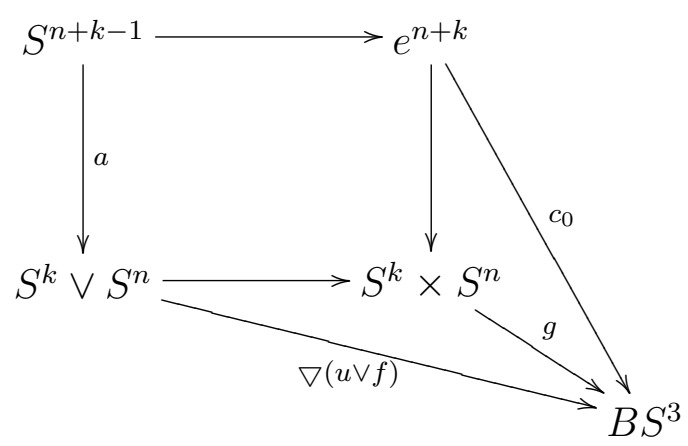

Com isso, a aplicação $g$ existe se, e somente se, $\nabla(u \vee f) a \sim c_{0}$. Mas $\nabla(u \vee f) a=$ $[[u],[f]]$ ([10] 10.47 pág. 319), e assim, precisamos calcular o produto de Whitehead

$$
[,]: \pi_{k}\left(B S^{3}\right) \times \pi_{n}\left(B S^{3}\right) \rightarrow \pi_{n+k-1}\left(B S^{3}\right)
$$

definido por

$$
[[u],[f]]: S^{n+k-1} \underset{a}{\longrightarrow} S^{k} \vee S_{\nabla(u \vee f)}^{n} B S^{3} .
$$

Portanto,

$$
\operatorname{Imev}_{k}=\left\{[u] \in \pi_{k}\left(B S^{3}\right) \mid[[u],[f]]=0 \in \pi_{n+k-1}\left(B S^{3}\right)\right\} .
$$

\section{Corolário 4.1.3.}

$$
\pi_{2}\left(\mathcal{G}_{f}\right) \approx \pi_{n+2}\left(S^{3}\right) / \operatorname{Im} \partial_{4}
$$

$e$

$$
\operatorname{Im} \partial_{4} \approx \mathbb{Z} / \operatorname{Imev} v_{4}
$$


Demonstração: Considere $k=3$. Na seqüência apresentada no Lema 4.1.2, temos a seqüência exata

$$
\pi_{3}\left(\mathcal{G}_{f}\right) \underset{e v_{4}}{\longrightarrow} \pi_{3}\left(S^{3}\right) \approx \mathbb{Z} \underset{\partial_{4}}{\longrightarrow} \pi_{n+2}\left(S^{3}\right) \longrightarrow \pi_{2}\left(\mathcal{G}_{f}\right) \underset{e v_{3}}{\longrightarrow} \pi_{2}\left(S^{3}\right)=0 .
$$

Assim,

$$
\pi_{2}\left(\mathcal{G}_{f}\right) \approx \pi_{n+2}\left(S^{3}\right) / \operatorname{Im} \partial_{4}
$$

e

$$
\operatorname{Im} \partial_{4} \approx \mathbb{Z} / \operatorname{Im} e v_{4}
$$

Reduzimos os cálculos nos grupos de homotopia de $S^{4}$ usando o Lema 4.1.1.

Lema 4.1.4. Para todos os $k, n \geq 4, \omega=[u] \in \pi_{k}\left(B S^{3}\right), \beta_{f}=[f] \in \pi_{n}\left(B S^{3}\right)$, $[[u],[f]]=\left[\omega, \beta_{f}\right]= \pm j_{n+k-1}\left(\left[\iota_{4}, \iota_{4}\right] \circ \Sigma^{4} \partial \omega \circ \Sigma^{k} \partial \beta_{f}\right)= \pm j_{n+k-1}\left(\left[\iota_{4}, \iota_{4}\right] \circ \Sigma^{4} \partial \beta_{f} \circ \Sigma^{n} \partial \omega\right)$, em $\pi_{n+k-1}\left(B S^{3}\right) \approx j_{n+k-1}\left(\pi_{n+k-1}\left(S^{4}\right)\right)$.

Demonstração: Como $j_{n}$ é uma aplicação sobrejetiva, pelo Lema 4.1.1, podemos estudar o problema equivalente para

$$
\begin{gathered}
{[,]:\left(j_{k}\left(\pi_{k}\left(S^{4}\right)\right)=\pi_{k}\left(B S^{3}\right)\right) \times\left(j_{n}\left(\pi_{n}\left(S^{4}\right)\right)=\pi_{n}\left(B S^{3}\right)\right) \rightarrow} \\
\rightarrow j_{n+k-1}\left(\pi_{n+k-1}\left(S^{4}\right)\right)=\pi_{n+k-1}\left(B S^{3}\right) .
\end{gathered}
$$

Para todo $\omega=[u] \in \pi_{k}\left(B S^{3}\right)$, existe $\omega^{\prime}=\Sigma \partial \omega$ em $\pi_{k}\left(S^{4}\right)$ tal que $\omega=j_{k}\left(\omega^{\prime}\right)$. Analogamente, como $\beta_{f}=[f] \in \pi_{n}\left(B S^{3}\right)$, existe algum elemento $\beta_{f}^{\prime}=\Sigma \partial \beta_{f} \in \pi_{n}\left(S^{4}\right)$ tal que $\beta_{f}=[f]=j_{n}(\beta)$.

Logo,

$$
\begin{gathered}
{[[u],[f]]=\left[\omega, \beta_{f}\right]=\left[j_{k}\left(\omega^{\prime}\right), j_{n}\left(\beta_{f}^{\prime}\right)\right]=j_{n+k-1}\left(\left[\omega^{\prime}, \beta_{f}^{\prime}\right]\right)=} \\
=j_{n+k-1}\left(\left[\iota_{4} \circ \Sigma \partial \omega, \iota_{4} \circ \Sigma \partial \beta_{f}\right]\right)=j_{n+k-1}\left(\left[\iota_{4}, \iota_{4}\right] \circ \Sigma\left(\partial \omega \wedge \partial \beta_{f}\right)\right),
\end{gathered}
$$

por [47] Cap. X 8.18, em $\pi_{n+k-1}\left(B S^{3}\right)$.

Agora, já que $\partial \omega \in \pi_{k-1}\left(S^{3}\right)$ e $\partial \beta_{f} \in \pi_{n-1}\left(S^{3}\right)$, se $k, n \geq 4$, por [47] Cap. X 8.12

$$
\partial \omega \wedge \partial \beta_{f}= \pm \Sigma^{3} \partial \omega \circ \Sigma^{3+k-1-3} \partial \beta_{f}
$$

e pela relação (1) do Apêndice temos

$$
\pm \Sigma^{3} \partial \omega \circ \Sigma^{3+k-1-3} \partial \beta_{f}=\Sigma^{3} \partial \beta_{f} \circ \Sigma^{3+n-1-3} \partial \omega
$$

em $\pi_{n+k-2}\left(S^{6}\right)$

Assim, nossa equação será

$$
[[u],[f]]=\left[\omega, \beta_{f}\right]= \pm j_{n+k-1}\left(\left[\iota_{4}, \iota_{4}\right] \circ \Sigma^{4} \partial \omega \circ \Sigma^{k} \partial \beta_{f}\right)=j_{n+k-1}\left(\left[\iota_{4}, \iota_{4}\right] \circ \Sigma^{4} \partial \beta_{f} \circ \Sigma^{n} \partial \omega\right),
$$

$\operatorname{em} \pi_{n+k-1}\left(B S^{3}\right) \approx j_{n+k-1}\left(\pi_{n+k-1}\left(S^{4}\right)\right)$.

Podemos simplificar a equação ainda mais, trabalhando com o produto de Whitehead. Algum cuidado é necessário, veja Apêndice no final do capítulo. 
Lema 4.1.5. Para todo $\psi \in \pi_{n}\left(S^{6}\right)$,

$$
j_{n+1}\left(\left[\iota_{4}, \iota_{4}\right] \circ \Sigma \psi\right)=j_{n+1} \Sigma(\xi \circ \psi),
$$

em $\pi_{n+1}\left(B S^{3}\right) \approx j_{n+1}\left(\pi_{n+1}\left(S^{4}\right)\right)$, com $\pi_{6}\left(S^{3}\right)=\mathbb{Z} / 12[\xi]$ e $j_{n+1}$ é injetiva sobre esses elementos.

Demonstração: Considere

$$
j_{7}\left(\left[\iota_{4}, \iota_{4}\right]\right)=\left[j_{4}\left(\iota_{4}\right), j_{4}\left(\iota_{4}\right)\right] .
$$

Por [17] 16.4 pág. 330,

$$
\left[\iota_{4}, \iota_{4}\right]=2 \nu_{4}-\Sigma \xi
$$

Logo

$$
\left[j_{4}\left(\iota_{4}\right), j_{4}\left(\iota_{4}\right)\right]=2 j_{7}\left(\nu_{4}\right)+j_{7}(\Sigma \xi) .
$$

Agora, $\nu_{4}$ está no núcleo de $j_{7}$ pelo Lema 4.4.1 do Apêndice no final do capítulo. Obtemos com isso

$$
j_{n+1}\left(\left[\iota_{4}, \iota_{4}\right] \circ \Sigma \psi\right)=j_{n+1}\left(\left(2 \nu_{4}-\Sigma \xi\right) \circ \Sigma \psi\right)=j_{n+1}(\Sigma \xi \circ \Sigma \psi)=j_{n+1} \Sigma(\xi \circ \psi),
$$

e pelo mesmo Lema sabemos que $j_{n+1}$ é injetiva sobre esses elementos.

Corolário 4.1.6. Para todo $k, n \geq 4, \omega=[u] \in \pi_{k}\left(B S^{3}\right), \beta_{f}=[f] \in \pi_{n}\left(B S^{3}\right)$,

$$
\left[\omega, \beta_{f}\right]= \pm j_{n+k-1}\left(\Sigma \xi \circ \Sigma^{4} \partial \omega \circ \Sigma^{k} \partial \beta_{f}\right)=j_{n+k-1}\left(\Sigma \xi \circ \Sigma^{4} \partial \beta_{f} \circ \Sigma^{n} \partial \omega\right)
$$

em $\pi_{n+k-1}\left(B S^{3}\right)=j_{n+k-1}\left(\pi_{n+k-1}\left(S^{4}\right)\right)$, com $\pi_{6}\left(S^{3}\right)=\mathbb{Z} / 12[\xi]$ e $j_{n+k-1}$ é injetiva sobre esses elementos.

Demonstração: Pelo Lema 4.1.4 temos

$$
\left[\omega, \beta_{f}\right]= \pm j_{n+k-1}\left(\left[\iota_{4}, \iota_{4}\right] \circ \Sigma^{4} \partial \omega \circ \Sigma^{k} \partial \beta_{f}\right)
$$

e observemos também que

$$
\Sigma^{4} \partial \omega \circ \Sigma^{k} \partial \beta_{f}=\Sigma\left(\Sigma^{3} \partial \omega \circ \Sigma^{k-1} \partial \beta_{f}\right)=\Sigma \psi
$$

para algum $\psi \in \pi_{n+k-2}\left(S^{6}\right)$.

Assim, pelo Lema 4.1.5,

$$
\begin{gathered}
j_{n+k-1}\left(\left[\iota_{4}, \iota_{4}\right] \circ \Sigma^{4} \partial \omega \circ \Sigma^{k} \partial \beta_{f}\right)=j_{n+k-1}\left(\left[\iota_{4}, \iota_{4}\right] \circ \Sigma \psi\right)= \\
j_{n+k-1}(\Sigma \xi \circ \Sigma \psi)=j_{n+k-1}\left(\Sigma \xi \circ \Sigma^{4} \partial \omega \circ \Sigma^{k} \partial \beta_{f}\right) .
\end{gathered}
$$

A segunda igualdade segue da relação (1) do Apêndice. 
Lema 4.1.7. Para todo $\psi \in \pi_{n}\left(S^{6} ; p\right), \operatorname{com} p \neq 2$,

$$
j_{n+1}\left(\left[\iota_{4}, \iota_{4}\right] \circ \Sigma \psi\right)=0
$$

$e m \pi_{n+1}\left(B S^{3}\right) \approx j_{n+1}\left(\pi_{n+1}\left(S^{4}\right)\right)$.

Demonstração: Se $\psi$ tem ordem $p^{k}$, então

$$
p^{k}\left[\iota_{4}, \iota_{4}\right] \circ \Sigma \psi=0 \in \pi_{n+1}\left(S^{4}\right)
$$

e conseqüentemente

$$
\left[\iota_{4}, \iota_{4}\right] \circ \Sigma \psi \in \pi_{n+1}\left(S^{4} ; p\right) \approx \pi_{n}\left(S^{3} ; p\right) \oplus \pi_{n+1}\left(S^{7} ; p\right),
$$

com o isomorfismo acima dado em [46] pág. 172 por

$$
\begin{gathered}
\pi_{n}\left(S^{3} ; p\right) \oplus \pi_{n+1}\left(S^{7} ; p\right) \rightarrow \pi_{n+1}\left(S^{4} ; p\right), \\
(x, y) \mapsto \Sigma x+\left[\iota_{4}, \iota_{4}\right] \circ y .
\end{gathered}
$$

Isso mostra que $\left[\iota_{4}, \iota_{4}\right] \circ \Sigma \psi \in\left[\iota_{4}, \iota_{4}\right] \circ \pi_{n+1}\left(S^{7}\right)$ e, assim, pertence ao núcleo de $j_{n+1}$, por razões análogas à prova do Lema 4.4.1 no Apêndice deste capítulo.

A partir das informações obtidas com os lemas anteriores obtemos a seguinte proposição:

Proposição 4.1.8. Sejam $f \in m\left(S^{n}, B S^{3} ; f\right), n \geq 5, \beta_{f}=[f] \in \pi_{n}\left(B S^{3}\right), \partial \beta_{f} \in$ $\pi_{n-1}\left(S^{3}\right)$. Decomponha $2 \beta_{f}=\partial \beta_{f}(2)+\partial \beta_{f}(p) \in \pi_{n-1}\left(S^{3} ; 2\right)+\sum_{p \neq 2} \pi_{n-1}\left(S^{3} ; p\right)$. Sejam $\omega=[u] \in \pi_{k}\left(B S^{3}\right), k>4, \partial \omega \in \pi_{k-1}\left(S^{3}\right), \partial \omega=\partial \omega(2)+\partial \omega(p) \in \pi_{k-1}\left(S^{3} ; 2\right)+$ $\sum_{p \neq 2} \pi_{k-1}\left(S^{3} ; p\right)$. Então temos a seqüência exata longa

$$
\begin{gathered}
\cdots \longrightarrow \pi_{k+1}\left(B S^{3}\right) \underset{\partial_{k+1}}{\longrightarrow} \pi_{k+n}\left(B S^{3}\right) \longrightarrow \\
\longrightarrow \pi_{k-1}\left(\mathcal{G}_{f}\right) \underset{e v_{k}}{\longrightarrow} \pi_{k}\left(B S^{3}\right) \underset{\partial_{k}}{\longrightarrow} \pi_{k+n-1}\left(B S^{3}\right) \longrightarrow \cdots
\end{gathered}
$$

e

$$
\begin{aligned}
\operatorname{Im} e v_{k} & =\operatorname{Nuc} \partial_{k}=\left\{\omega \in \pi_{k}\left(B S^{3}\right) \mid \nu^{\prime} \circ \Sigma^{3} \partial \omega(2) \circ \Sigma^{k-1} \partial \beta_{f}(2)=\right. \\
& \left.=\nu^{\prime} \circ \Sigma^{3} \partial \beta_{f}(2) \circ \Sigma^{n-1} \partial \omega(2)=0 \in \pi_{n+k-2}\left(S^{3} ; 2\right)\right\},
\end{aligned}
$$

$\operatorname{com} \pi_{6}\left(S^{3} ; 2\right)=\mathbb{Z} / 4\left[\nu^{\prime}\right]$.

O mesmo pode ser lido em termos dos grupos de homotopia de $S^{3}$ como segue:

$$
\begin{gathered}
\cdots \longrightarrow \pi_{k}\left(S^{3}\right) \underset{\partial_{k+1}}{\longrightarrow} \pi_{k+n-1}\left(S^{3}\right) \longrightarrow \\
\longrightarrow \pi_{k-1}\left(\mathcal{G}_{f}\right) \underset{e v_{k}}{\longrightarrow} \pi_{k-1}\left(S^{3}\right) \underset{\partial_{k}}{\longrightarrow} \pi_{k+n-2}\left(S^{3}\right) \longrightarrow \cdots
\end{gathered}
$$

e

$$
\begin{aligned}
\operatorname{Im} e v_{k} & =\operatorname{Nuc} \partial_{k}=\left\{\partial \omega \in \pi_{k-1}\left(S^{3}\right) \mid \nu^{\prime} \circ \Sigma^{3} \partial \omega(2) \circ \Sigma^{k-1} \partial \beta_{f}(2)=\right. \\
& \left.=\nu^{\prime} \circ \Sigma^{3} \partial \beta_{f}(2) \circ \Sigma^{n-1} \partial \omega(2)=0 \in \pi_{n+k-2}\left(S^{3} ; 2\right)\right\} .
\end{aligned}
$$

\footnotetext{
${ }^{2}$ Isso pode ser feito porque sabemos que os grupos de homotopia de $S^{3}$, em dimensão grande, são finitos.
} 
Demonstração: A equação do Lema 4.1.2 torna-se

$$
0=[[u],[f]]=\left[\omega, \beta_{f}\right]=j_{n+k-1}\left(\left[\iota_{4}, \iota_{4}\right] \circ \Sigma^{4} \partial \omega \circ \Sigma^{k} \partial \beta_{f}\right),
$$

pelo Lema 4.1.4, e com isso

$$
\begin{gathered}
0=j_{n+k-1}\left(\left[\iota_{4}, \iota_{4}\right] \circ \Sigma^{4} \partial \omega(2) \circ \Sigma^{k} \partial \beta_{f}(2)\right)+\sum_{p \neq 2} j_{n+k-1}\left(\left[\iota_{4}, \iota_{4}\right] \circ \Sigma^{4} \partial \omega(p) \circ \Sigma^{k} \partial \beta_{f}(p)\right)= \\
=j_{n+k-1} \Sigma\left(\nu^{\prime} \circ \Sigma^{3} \partial \omega(2) \circ \Sigma^{k-1} \partial \beta_{f}(2)\right),
\end{gathered}
$$

em $\pi_{n+k-1}\left(B S^{3}\right) \approx j_{n+k-1}\left(\pi_{n+k-1}\left(S^{4}\right)\right)$ pelos Lemas 4.1.5 e 4.1.7. Pelo Lema 4.1.5, $j_{n+k-1}$ é injetiva sobre esses elementos e a tese segue. Note que a equação pode ser considerada como

$$
\Sigma \nu^{\prime} \circ \Sigma^{4} \partial \omega(2) \circ \Sigma^{k} \partial \beta_{f}(2)=0,
$$

em $\Sigma\left(\pi_{n+k-2}\left(S^{3} ; 2\right)\right) \subseteq \pi_{n+k-1}\left(S^{4} ; 2\right)$, ou como enunciado no Lema 4.1.2.

Quando $k=4$, temos isomorfismos $\pi_{3}\left(S^{3}\right) \approx \pi_{4}\left(B S^{3}\right) \approx \pi_{4}\left(S^{4}\right)=\mathbb{Z}$, com gerador de ordem infinita. Com as modificações, o resultado fica da seguinte forma:

Proposição 4.1.9. Sejam $f \in m\left(S^{n}, B S^{3} ; f\right), n \geq 5, \beta_{f}=[f] \in \pi_{n}\left(B S^{3}\right), \partial \beta_{f} \in$ $\pi_{n-1}\left(S^{3}\right)$. Decomponha $\partial \beta_{f}=\partial \beta_{f}(2)+\partial \beta_{f}(p) \in \pi_{n-1}\left(S^{3} ; 2\right)+\sum_{p \neq 2} \pi_{n-1}\left(S^{3} ; p\right)$. Então

$\operatorname{Im} e v_{4}=\operatorname{Nuc} \partial_{4}=\left\{m \in \mathbb{Z} \mid m \nu^{\prime} \circ \Sigma^{3} \partial \beta_{f}(2)=0 \in \pi_{n+2}\left(S^{3} ; 2\right)\right\}$,

$\operatorname{com} \pi_{6}\left(S^{3} ; 2\right)=\mathbb{Z} / 4\left[\nu^{\prime}\right]$.

Demonstração: Da Proposição 4.1 .8 segue que

$$
\begin{gathered}
\operatorname{Im} e v_{4}=\left\{m j_{4}\left(\iota_{4}\right) \in \pi_{4}\left(B S^{3}\right) \approx \mathbb{Z}\left[j_{4}\left(\iota_{4}\right)\right], m \in \mathbb{Z} \mid\right. \\
\left.\mid m \Sigma \nu^{\prime} \circ \Sigma^{4} \partial \beta_{f}(2)=0 \in \Sigma\left(\pi_{n+2}\left(S^{3} ; 2\right)\right) \subseteq \pi_{n+3}\left(S^{4}\right)\right\} .
\end{gathered}
$$

Lema 4.1.10. $\pi_{2}\left(\mathcal{G}_{c_{0}}\right) \approx \pi_{n+2}\left(S^{3}\right)$.

Demonstração: Como $[f]=\left[c_{0}\right]$ no caso presente, todos os elementos $n \iota_{4}$ pertencem à imagem de $e v_{4}$, pelo Lema 4.1.2, então $\operatorname{Im} e v_{4}=\mathbb{Z}$. A tese segue do Corolário 4.1.3.

\subsection{Alguns resultados sobre os grupos de homotopia das fibras}

A segunda parte do problema é estabelecer quantos diferentes tipos de homotopia temos para o grupo de gauge $\mathcal{G}_{f}$. Ele é um subconjunto do conjunto das fibras de homotopia aparecendo nas fibrações

$$
F_{h} \longrightarrow S^{3} \longrightarrow \Omega_{0}^{n-1} S^{3},
$$


sendo $h$ qualquer aplicação em qualquer uma das classes $[h] \in \pi_{3}\left(\Omega_{0}^{n-1} S^{3}\right) \approx \pi_{n+2}\left(S^{3}\right)$. Assim, nosso objetivo é enumerar os elementos do conjunto

$$
\left\{\left[F_{h}\right], h: S^{3} \rightarrow \Omega_{0}^{n-1} S^{3}\right\}
$$

Como duas aplicações na mesma classe têm o mesmo tipo, isto é, $h \sim h^{\prime}$ implica $F_{h} \sim F_{h^{\prime}}$, podemos considerar o conjunto equivalente

$$
\left\{\left[F_{h}\right],[h] \in \pi_{n+2}\left(S^{3}\right)\right\}
$$

o qual é isomorfo ao conjunto das classes dos elementos de $\pi_{n+2}\left(S^{3}\right)$ e, assim, considerando apenas o representante de um subconjunto de $\pi_{n+2}\left(S^{3}\right)^{3}$.

Uma maneira para distingüir os diferentes tipos para $F_{h}$ é encontrar alguns diferentes grupos de homotopia. Se temos suficientes valores diferentes para esses grupos de homotopia para cobrir todos os elementos em $\pi_{n+2}\left(S^{3}\right)$, então o trabalho está feito. Começaremos considerando $\pi_{2}\left(F_{h}\right)$.

Lema 4.2.1. Seja $h: S^{3} \rightarrow \Omega_{0}^{n-1} S^{3}$ e considere a fibração

$$
F_{h} \rightarrow S^{3} \rightarrow \Omega_{0}^{n-1} S^{3}
$$

O homomorfismo induzido por $h$ na seqüência de homotopia

$$
\underset{\partial_{k+1}}{\longrightarrow} \pi_{k}\left(F_{h}\right) \underset{i_{k}}{\longrightarrow} \pi_{k}\left(S^{3}\right) \underset{h_{k}}{\longrightarrow} \pi_{k+n-1}\left(S^{3}\right) \underset{\partial_{k}}{\longrightarrow}
$$

é dado como segue:

$$
h_{k}(\zeta)=\beta \circ \Sigma^{n-1} \zeta,
$$

sendo $\beta=[\operatorname{ad}(h)] \in \pi_{n+2}\left(S^{3}\right)$ e $\zeta=[f] \in \pi_{k}\left(S^{3}\right)$.

Demonstração: Considere $h: S^{3} \rightarrow \Omega_{0}^{n-1} S^{3}$. Então,

$$
\begin{gathered}
h_{k}: \pi_{k}\left(S^{3}\right) \rightarrow \pi_{k}\left(\Omega_{0}^{n-1} S^{3}\right), \\
h_{k}:[f] \mapsto[h f] .
\end{gathered}
$$

Os isomorfismos adjuntos são

$$
\begin{aligned}
a d: m_{0}\left(S^{3}, \Omega_{0}^{n-1} S^{3}\right) & \rightarrow m_{0}\left(S^{3} \wedge S^{n-1}, S^{3}\right), \\
a d: h \mapsto \widetilde{h} &
\end{aligned}
$$

$\operatorname{com} \tilde{h}(x \wedge y)=h(x)(y) \mathrm{e}$

$$
a d: m_{0}\left(S^{k}, \Omega_{0}^{n-1} S^{3}\right) \rightarrow m_{0}\left(S^{k} \wedge S^{n-1}, S^{3}\right)
$$

\footnotetext{
${ }^{3} \mathrm{Na}$ verdade, perdemos algo considerando as classes baseadas e não as classes livres, mas conhecemos o conjunto das classes baseadas, $\pi_{n+2}\left(S^{3}\right)$, enquanto, em geral, não conhecemos o conjunto das classes livres.
} 


$$
a d:(h f) \mapsto \widetilde{h f} .
$$

Agora

$$
\widetilde{h f}(z \wedge y)=h f(z)(y)=h(f(z))(y)=\widetilde{h}(f(z) \wedge y) .
$$

Então,

$$
\operatorname{ad}(h f)=\operatorname{ad}(h)(f \wedge 1) .
$$

Assim, se $h: S^{3} \rightarrow \Omega_{0}^{n-1} S^{3}, \operatorname{ad}(h): S^{3} \wedge S^{n-1} \rightarrow S^{3}$, isto é, $[\operatorname{ad}(h)] \in \pi_{n+2}\left(S^{3}\right)$. Para cada $[f] \in \pi_{k}\left(S^{3}\right),[h f] \in \pi_{k}\left(\Omega_{0}^{n-1} S^{3}\right)$ e $[a d(h f)]=[\operatorname{ad}(h)(f \wedge 1)] \in \pi_{k+n-1}\left(S^{3}\right)$.

Então,

$$
\begin{gathered}
h_{k}: \pi_{k}\left(S^{3}\right) \rightarrow \pi_{k+n-1}\left(S^{3}\right), \\
h_{k}:[f] \mapsto[a d(h f)]=[\operatorname{ad}(h)] \circ\left([f] \wedge \iota_{n-1}\right)=[\operatorname{ad}(h)] \circ \Sigma^{n-1}[f] .
\end{gathered}
$$

Em diagramas,

$$
\begin{gathered}
S^{k} \underset{f}{\longrightarrow} S^{3} \underset{h}{\longrightarrow} \Omega_{0}^{n-1} S^{3}, \\
S^{k} \wedge S^{n-1} \underset{f \wedge 1}{\longrightarrow} S^{3} \wedge S_{a d(h)}^{n-1} S^{3} .
\end{gathered}
$$

Se denotarmos por $\beta$ as classes de $a d(h)$ e por $\zeta$ as de $f$, temos

$$
h_{k}(\zeta)=\beta \circ \Sigma^{n-1} \zeta
$$

Lema 4.2.2. Para todo $[h] \in \pi_{n+2}\left(S^{3}\right)$,

$$
\pi_{2}\left(F_{h}\right) \approx \pi_{n+2}\left(S^{3}\right) / \operatorname{Im} h_{3},
$$

sendo $h_{3}$ o homomorfismo induzido por h na seqüência de homotopia da fibração induzida por $h: S^{3} \rightarrow \Omega_{0}^{n-1} S^{3}$,

$$
\mathbb{Z} \underset{h_{3}}{\longrightarrow} \pi_{n+2}\left(S^{3}\right) \underset{\partial_{3}}{\longrightarrow} \pi_{2}\left(F_{h}\right) \underset{i_{2}}{\longrightarrow} 0
$$

e $h_{3} \in \operatorname{Hom}\left(\mathbb{Z}, \pi_{n+2}\left(S^{3}\right)\right) \approx \pi_{n+2}\left(S^{3}\right)$.

Demonstração: Recorde que começamos com a fibração

$$
F_{h} \underset{i}{\longrightarrow} S^{3} \underset{h}{\longrightarrow} \Omega_{0}^{n-1} S^{3}
$$

sendo $h$ qualquer aplicação em qualquer uma das classes $[h] \in \pi_{3}\left(\Omega_{0}^{n-1} S^{3}\right) \approx \pi_{n+2}\left(S^{3}\right)$. Considerando a seqüência exata de homotopia associada à $h$

$$
\begin{gathered}
\cdots \underset{i_{3}}{\cdots} \underset{i_{3}}{\longrightarrow} \pi_{3}\left(S^{3}\right) \approx \mathbb{Z} \underset{h_{3}}{\longrightarrow} \pi_{3}\left(\Omega_{0}^{n-1} S^{3}\right) \underset{\partial_{3}}{\longrightarrow} \\
\underset{\partial_{3}}{\longrightarrow} \pi_{2}\left(F_{h}\right) \underset{i_{2}}{\longrightarrow} \pi_{2}\left(S^{3}\right)=0 \underset{h_{2}}{\longrightarrow} \pi_{2}\left(\Omega_{0}^{n-1} S^{3}\right) \underset{\partial_{2}}{\longrightarrow}
\end{gathered}
$$




$$
\underset{\partial_{2}}{\longrightarrow} \pi_{1}\left(F_{h}\right) \longrightarrow \pi_{1}\left(S^{3}\right)=0 \underset{h_{1}}{\longrightarrow} \cdots
$$

obtemos a seqüência exata

$$
\mathbb{Z} \underset{h_{3}}{\longrightarrow} \pi_{n+2}\left(S^{3}\right) \underset{\partial_{3}}{\longrightarrow} \pi_{2}\left(F_{h}\right) \underset{i_{2}}{\longrightarrow} 0
$$

o que implica

$$
\pi_{2}\left(F_{h}\right) \approx \operatorname{Im} \partial_{3} \approx \pi_{n+2}\left(S^{3}\right) / \operatorname{Nuc} \partial_{3} \approx \pi_{n+2}\left(S^{3}\right) / \operatorname{Im} h_{3}
$$

Lema 4.2.3. Seja $h=c_{0}$ a aplicação trivial. Então $\pi_{2}\left(F_{h=c_{0}}\right) \approx \pi_{n+2}\left(S^{3}\right)$.

Demonstração: Se $h=c_{0}$ é aplicação trivial, então $h_{3}=\left(c_{0}\right)_{3}=0$ é o homomorfismo trivial e $\operatorname{Im} h_{3}=0$. Então, $\pi_{2}\left(F_{h}\right) \approx \pi_{n+2}\left(S^{3}\right) / \operatorname{Im} h_{3} \approx \pi_{n+2}\left(S^{3}\right)$.

Lema 4.2.4. Para todo $n>4, \pi_{2}\left(F_{h}\right) \approx \pi_{2}\left(F_{c_{0}}\right) \approx \pi_{n+2}\left(S^{3}\right)$ se, e somente se, $h \sim c_{0}$.

Demonstração: Recordemos que todos os grupos envolvidos são grupos abelianos finitos. Como $\pi_{2}\left(F_{h}\right) \approx \pi_{n+2}\left(S^{3}\right) / \operatorname{Im} h_{3}$, se $\pi_{2}\left(F_{c_{0}}\right) \approx \pi_{n+2}\left(S^{3}\right)$,então $\operatorname{Im} h_{3}=0$.

Agora, $h_{3} \in \operatorname{Hom}\left(\mathbb{Z}, \pi_{n+2}\left(S^{3}\right)\right)$. Considere $h_{3}(1) \in \operatorname{Im} h_{3}$. Já que $h_{3}(1) \neq 0$ sempre que $h_{3} \neq 0$, segue que $\operatorname{Im} h_{3} \neq 0$ para todo $h_{3} \neq 0$. Logo, se $\operatorname{Im} h_{3}=0$, temos $h_{3}=0$. Também, $\operatorname{como} \operatorname{Hom}\left(\mathbb{Z}, \pi_{n+2}\left(S^{3}\right)\right) \approx \pi_{n+2}\left(S^{3}\right), h_{3}=0$ se, e somente se, $[h]=0 \mathrm{em}$ $\pi_{n+2}\left(S^{3}\right)$. E como existe uma homotopia baseada, certamente $h \sim c_{0}$ livremente, como desejado.

Lema 4.2.5. $\pi_{j}\left(\mathcal{G}_{c_{0}}\right) \approx \pi_{j}\left(S^{3}\right) \oplus \pi_{j+n}\left(S^{3}\right)$.

Demonstração: Como $S^{3}$ é um grupo, $m\left(S^{n}, S^{3}\right)=m_{0}\left(S^{n}, S^{3}\right) \times S^{3}$ (veja [24]). E como $\mathcal{G}_{c_{0}} \sim m\left(S^{n}, S^{3}\right)$, o resultado segue.

Lema 4.2.6. $\mathcal{G}_{c_{0}} \sim F_{c_{0}}$.

Demonstração: Considere a fibração

$$
F_{c_{0}} \longrightarrow S^{3} \underset{c_{0}}{\longrightarrow} \Omega_{0}^{n-1} S^{3} .
$$

Como $c_{0}$ induz o homomorfismo trivial, obtemos a seqüência exata de homotopia para todo $j$,

$$
0 \longrightarrow \pi_{j+n}\left(S^{3}\right) \longrightarrow \pi_{j}\left(F_{c_{0}}\right) \longrightarrow \pi_{j}\left(S^{3}\right) \longrightarrow 0
$$

e, assim, os dois espaços possuem todos os grupos de homotopia isomorfos. Por outro lado, sabemos que $\mathcal{G}_{c_{0}}$ deve ser homotópico a $F_{h}$ para alguma $h$ e provamos no Lema 4.2 .4 que a única $F_{h} \operatorname{com} \pi_{2}$ igual a de $F_{c_{0}}$ é, na verdade, a própria $F_{c_{0}}$, e isso completa a prova. 
Lema 4.2.7. Se $\pi_{2}\left(\mathcal{G}_{f}\right) \approx \pi_{2}\left(\mathcal{G}_{c_{0}}\right)\left(\approx \pi_{n+2}\left(S^{3}\right)\right)$, então $\mathcal{G}_{f} \sim \mathcal{G}_{c_{0}}\left(\sim m\left(S^{2}, S^{3}\right)\right)$.

Demonstração: Necessariamente, $\mathcal{G}_{f} \sim \mathcal{G}_{h}$ para alguma $[h] \in \pi_{n+2}\left(S^{3}\right)$. Logo, $\pi_{2}\left(\mathcal{G}_{f}\right) \approx$ $\pi_{2}\left(F_{h}\right) \approx \pi_{n+2}\left(S^{3}\right)$ e, pelo Lema 4.2 .4 , temos $F_{h} \sim F_{c_{0}} \sim \mathcal{G}_{c_{0}}$.

Exemplo 4.2.8. Consideremos $n=2$ e $k=14$. Temos a seguinte seqüência exata

$$
\underset{\partial_{15}}{\longrightarrow} \pi_{14}\left(F_{h}\right) \underset{i_{14}}{\longrightarrow} \pi_{14}\left(S^{3}\right) \underset{h_{14}}{\longrightarrow} \pi_{15}\left(S^{3}\right) \underset{\partial_{14}}{\longrightarrow}
$$

$\operatorname{com} h_{14}(\zeta)=\operatorname{ad}(h) \circ \Sigma \zeta$ e obtemos $\pi_{2}\left(F_{h}\right) \approx \pi_{n+2}\left(S^{3}\right) / \operatorname{Im} h_{3}$ e $h_{3} \in \operatorname{Hom}\left(\mathbb{Z}, \pi_{n+2}\left(S^{3}\right)\right)$. Também, $a d(h) \in \pi_{16}\left(S^{3}\right)=\mathbb{Z} / 2\left[a=\nu^{\prime} \circ \eta_{6} \circ \mu_{7}\right] \oplus \mathbb{Z} / 3[\beta]$, e calculamos $\pi_{2}\left(F_{h}\right) \approx$ $\pi_{16}\left(S^{3}\right) / \operatorname{Im} h_{3}$. Como $h_{3}$ aplica $m \iota_{3}$ em $\operatorname{mad}(h)$, obtemos

$$
\begin{array}{cc}
a d(h) & \operatorname{Im} h_{3} \\
0 & 0 \\
a & \mathbb{Z} / 2[a] \\
\beta & \mathbb{Z} / 3[\beta] \\
2 \beta=-\beta & \mathbb{Z} / 3[\beta] \\
a+\beta & \mathbb{Z} / 6 \\
a+2 \beta=a-\beta & \mathbb{Z} / 6
\end{array}
$$

Logo, existe no máximo 6 tipos . Na verdade, as duas fibras $F_{ \pm \beta}$ e $F_{a \pm \beta}$ também têm o mesmo tipo. Podemos usar a equivalência de homotopia determinada sobre $\Omega^{13}\left(S^{3}\right)$ pelo laço inverso, e observe que isso pode ser identificado com a inversão do primo 3 . Então existem exatamente 4 tipos diferentes.

\subsection{Os tipos de homotopia dos grupos de gauge}

Para calcular quantos tipos diferentes de homotopia existem dos grupos de gauge de todos os $S^{3}$-fibrados principais sobre $S^{n}$, começamos calculando o $\pi_{2}$ desses grupos e, caso essa análise não seja suficente, continuamos os cálculos sobre grupos de homotopia em níveis maiores (como será necessário para os casos $n=13,21$ ).

4.3.1 Os casos $5 \leq n \leq 12, n=16,17,18,23,24,25$

Proposição 4.3.1. Para cada $n$ fixado com $n=7,8,9,10,11,15,16,17,18,23,24,25$, o tipo de homotopia do grupo de gauge de todos os $S^{3}$-fibrados principais sobre $S^{n}$ é o mesmo, mais ainda, é o mesmo do fibrado trivial, a saber $m\left(S^{n}, S^{3}\right) \sim \Omega_{0}^{n} S^{3} \times S^{3}$.

Demonstração: Para calcular o $\pi_{2}$ desses grupos de gauge precisamos encontrar $\operatorname{Im} e v_{4}$, segundo o Corolário 4.1.3 (usamos também a Tabela 1 apresentada no início do capítulo), e observe também que, pela Proposição 4.1.9, precisamos considerar apenas a 2-componente dos grupos de homotopia de $S^{3}$. Feito isso, veremos que em todos esses casos $\operatorname{Im} e v_{4}=\mathbb{Z}$. Então $\operatorname{Im} \partial_{4}=0$ e $\pi_{2}\left(\mathcal{G}_{f}\right) \approx \pi_{n+2}\left(S^{3}\right) \approx \pi_{2}\left(\mathcal{G}_{c_{0}}\right)$. 
Caso $n=7$. Temos que os fibrados sobre $S^{7}$ são classificados pelas classes

$$
\partial \beta_{f} \in \pi_{6}\left(S^{3}\right)=\mathbb{Z} / 12[\xi] .
$$

Então, pela Tabela 1 do início do capítulo, temos 12 fibrados $f_{k}=k \xi$ sobre $S^{7}$ (número de fibrados dado pela ordem do grupo), e assim teremos no máximo 12 diferentes tipos de homotopia para os grupos de gauge. Como precisamos calcular inicialmente Im $e v_{4}$, para posteriormente calcularmos o $\pi_{2}$ do grupo de gauge (Corolário 4.1.3), utilizamos a Proposição 4.1 .9 e por ela vemos que basta considerarmos a 2-componente de $\pi_{6}\left(S^{3}\right)=$ $\mathbb{Z} / 12[\xi]$ (decompondo $\xi$ sobre as 2, 3-componentes, temos $\xi=3 \nu^{\prime}+4 \alpha_{1}(3 ; 3)$ ). Assim, precisamos encontrar para quais valores de $m \in \mathbb{Z}$ a igualdade

$$
m \Sigma \nu^{\prime} \circ \Sigma^{4} \nu^{\prime}=0
$$

é verdadeira. Mas, observemos que

$$
\Sigma \nu^{\prime} \circ \Sigma^{4} \nu^{\prime}=\Sigma\left(\nu^{\prime} \circ 2 \nu_{6}\right)=0
$$

por [46] 5.4 pág. 41 e pág. 51 (o elemento $\nu^{\prime} \circ \nu_{6}$ tem ordem 4 e pertence a $\pi_{9}\left(S^{3}\right)$, o qual não possui elementos de ordem par).

Assim, $\operatorname{Im} e v_{4}=\mathbb{Z}$ para toda $f$, e com isso $\operatorname{Im} \partial_{4}=0$ e $\pi_{2}\left(\mathcal{G}_{f}\right) \approx \pi_{9}\left(S^{3}\right) \approx \pi_{2}\left(\mathcal{G}_{c_{0}}\right)$, sendo o último isomorfismo dado pelo Lema 4.1 .10 e temos, pelo Lema 4.2 .7 , que $\mathcal{G}_{f} \sim \mathcal{G}_{c_{0}}$ para toda $f$.

Caso $n=8$. Analogamente ao que fizemos no caso anterior, consideremos

$$
\partial \beta_{f} \in \pi_{7}\left(S^{3}\right)=\mathbb{Z} / 2\left[\xi \circ \eta_{6}\right]
$$

Então temos 2 fibrados $f_{0}=c_{0}$ e $\partial\left[f_{1}\right]=\xi \circ \eta_{6}$. Pela Proposição 4.1.9, precisamos considerar apenas

$$
\Sigma \nu^{\prime} \circ \Sigma^{4}\left(\nu^{\prime} \circ \eta_{6}\right)
$$

Do fato de que $\nu^{\prime} \circ \eta_{6}=\eta_{3} \circ \nu_{4}$, por [46] 5.9 pág. 44, obtemos

$$
\Sigma \nu^{\prime} \circ \Sigma^{4}\left(\eta_{3} \circ \nu_{4}\right)=\Sigma \nu^{\prime} \circ \eta_{7} \circ \nu_{8}=0,
$$

já que $\eta_{7} \circ \nu_{8}=0$, por [46] 5.9 pág. 44. Assim, $\operatorname{Im} e v_{4}=\mathbb{Z}$ para toda $f$ e com isso $\pi_{2}\left(\mathcal{G}_{f}\right) \approx \pi_{10}\left(S^{3}\right)$ para toda $f$.

Caso $n=9$.

$$
\partial \beta_{f} \in \pi_{8}\left(S^{3}\right)=\mathbb{Z} / 2\left[\nu^{\prime} \circ \eta_{6}^{2}\right] .
$$

Então temos 2 fibrados $f_{0}=c_{0}$ e $\partial\left[f_{1}\right]=\xi \circ \eta_{6}^{2}$. Pela Proposição $4.1 .9(\operatorname{com} k=4)$, precisamos considerar apenas

$$
\nu^{\prime} \circ \Sigma^{3}\left(\nu^{\prime} \circ \eta_{6} \circ \eta_{7}\right) .
$$

Recordemos que $\nu^{\prime} \circ \eta_{6}=\eta_{3} \circ \nu_{4}([46] 5.9$ pág. 44), e obtemos

$$
\nu^{\prime} \circ \Sigma^{3}\left(\eta_{3} \circ \nu_{4} \circ \eta_{7}\right)=\nu^{\prime} \circ \eta_{6} \circ \nu_{7} \circ \eta_{10}=0,
$$


como $\eta_{6} \circ \nu_{7}=0$ por [46] 5.9 pág. 44. Assim, Im $e v_{4}=\mathbb{Z}$ para toda $f$ e com isso $\pi_{2}\left(\mathcal{G}_{f}\right) \approx \pi_{11}\left(S^{3}\right)$, para toda $f$.

Casos $n=10,11$.

Já que

$$
\partial \beta_{f} \in \pi_{9}\left(S^{3}\right) \approx \mathbb{Z} / 3 \text { ou } \pi_{10}\left(S^{3}\right) \approx \mathbb{Z} / 15
$$

temos que a 2-componente $\partial \beta_{f}(2)=0$. Assim, pela Proposição 4.1 .9 obtemos $\operatorname{Im} e v_{4}=\mathbb{Z}$ para toda $f$ e com isso $\pi_{2}\left(\mathcal{G}_{f}\right) \approx \pi_{12}\left(S^{3}\right)$ ou $\pi_{13}\left(S^{3}\right)$ para toda $f$, respectivamente quando $n=10,11$.

Caso $n=15$.

$$
\begin{gathered}
\partial \beta_{f} \in \pi_{14}\left(S^{3}\right) \approx \mathbb{Z} / 84 \oplus \mathbb{Z} / 2 \oplus \mathbb{Z} / 2 \\
\partial \beta_{f}(2) \in \pi_{14}\left(S^{3} ; 2\right)=\mathbb{Z} / 4\left[\mu^{\prime}\right] \oplus \mathbb{Z} / 2\left[\epsilon_{3} \circ \nu_{4}\right] \oplus \mathbb{Z} / 2\left[\nu^{\prime} \circ \epsilon_{6}\right] .
\end{gathered}
$$

Então temos 336 fibrados. Pela Proposição 4.1 .9 (com $k=4$ ) precisamos considerar apenas

$$
\nu^{\prime} \circ \Sigma^{3} \mu^{\prime}=\nu^{\prime} \circ 2 \zeta_{6}=0,
$$

pois $\nu^{\prime} \circ \zeta_{6} \in \pi_{17}\left(S^{3} ; 2\right) \approx \mathbb{Z} / 2 \mathrm{e}$

$$
\nu^{\prime} \circ \Sigma^{3}\left(\epsilon_{3} \circ \nu_{4}\right)=\nu^{\prime} \circ \epsilon_{6} \circ \nu_{7} \stackrel{(1)}{=}{ }^{4} \nu^{\prime} \circ \nu_{6} \circ \epsilon_{9}=0,
$$

já que $\nu^{\prime} \circ \nu_{6} \in \pi_{9}^{3}=0$. Usando o mesmo argumento e a relação (5) do Apêndice deste capítulo,

$$
\nu^{\prime} \circ \Sigma^{3}\left(\nu^{\prime} \circ \epsilon_{6}\right)=\nu^{\prime} \circ 2 \nu_{6} \circ \epsilon_{9}=0,
$$

em $\pi_{17}\left(S^{3} ; 2\right)=\mathbb{Z} / 2\left[\epsilon_{3} \circ \nu_{11}^{2}\right]$. Assim, temos $\operatorname{Im} e v_{4}\left(\left[f_{\left[\mu^{\prime}\right]}\right]^{5}\right)=\operatorname{Im} e v_{4}\left(\left[f_{\left[\epsilon_{3} \circ \nu_{4}\right]}\right]\right)=$ $\operatorname{Im} e v_{4}\left(\left[f_{\left[\nu^{\prime} \circ \epsilon_{6}\right]}\right]\right)=\mathbb{Z}$. Segue então que

$$
\pi_{2}\left(\mathcal{G}_{f_{\left[\mu^{\prime}\right]}}\right) \approx \pi_{2}\left(\mathcal{G}_{f_{\left[\epsilon_{3} \circ \nu_{4}\right]}}\right) \approx \pi_{2}\left(\mathcal{G}_{f_{\left[\nu^{\prime} \circ \epsilon_{6}\right]}}\right) \approx \pi_{2}\left(\mathcal{G}_{c_{0}}\right) \approx \pi_{17}\left(S^{3}\right) .
$$

Caso $n=16$.

$$
\begin{gathered}
\partial \beta_{f} \in \pi_{15}\left(S^{3}\right) \approx \mathbb{Z} / 2 \oplus \mathbb{Z} / 2, \\
\partial \beta_{f}(2) \in \pi_{15}\left(S^{3} ; 2\right)=\mathbb{Z} / 2\left[\nu^{\prime} \circ \mu_{6}\right] \oplus \mathbb{Z} / 2\left[\nu^{\prime} \circ \eta_{6} \circ \epsilon_{7}\right] .
\end{gathered}
$$

Então temos 4 fibrados. Pela Proposição 4.1 .9 (com $k=4$ ) precisamos considerar apenas

$$
\nu^{\prime} \circ \Sigma^{3}\left(\nu^{\prime} \circ \mu_{6}\right) \stackrel{(5)}{=} \nu^{\prime} \circ 2 \nu_{6} \circ \mu_{9}=0,
$$

já que $\nu^{\prime} \circ \nu_{6} \in \pi_{9}^{3}=0$. Usando o mesmo argumento e a relação (5),

$$
\nu^{\prime} \circ \Sigma^{3}\left(\nu^{\prime} \circ \eta_{6} \circ \epsilon_{7}\right)=\nu^{\prime} \circ 2 \nu_{6} \circ \eta_{9} \circ \epsilon_{10}=0,
$$

\footnotetext{
${ }^{4}$ Os números indicados nas igualdades se referem as relações que podem ser encontradas no Apêndice deste capítulo.

${ }^{5}$ Como temos diferentes fibrados, indicaremos as classes para que não ocorram confusões.
} 
em $\pi_{18}\left(S^{3} ; 2\right)=\mathbb{Z} / 2\left[\bar{\epsilon}_{3}\right]$. Temos assim, $\operatorname{Im} e v_{4}\left(\left[f_{\left[\nu^{\prime} \circ \mu_{6}\right]}\right]\right)=\operatorname{Im} e v_{4}\left(\left[f_{\left[\nu^{\prime} \circ \eta_{6} \circ \epsilon_{7}\right]}\right]\right)=\mathbb{Z}$. Segue então que

$$
\pi_{2}\left(\mathcal{G}_{f_{\left[\nu^{\prime} \circ \mu_{6}\right]}}\right) \approx \pi_{2}\left(\mathcal{G}_{f_{\left[\nu^{\prime} \circ \eta_{6} \circ \epsilon_{7}\right]}}\right) \approx \pi_{2}\left(\mathcal{G}_{c_{0}}\right) \approx \pi_{18}\left(S^{3}\right)
$$

Caso $n=17$.

$$
\begin{gathered}
\partial \beta_{f} \in \pi_{16}\left(S^{3}\right) \approx \mathbb{Z} / 6, \\
\partial \beta_{f}(2) \in \pi_{16}\left(S^{3} ; 2\right)=\mathbb{Z} / 6\left[\nu^{\prime} \circ \eta_{6} \circ \mu_{7}\right] .
\end{gathered}
$$

Então temos 6 fibrados. Pela Proposição 4.1 .9 (com $k=4$ ) precisamos considerar apenas

$$
\nu^{\prime} \circ \Sigma^{3}\left(\nu^{\prime} \circ \eta_{6} \circ \mu_{7}\right) \stackrel{(5)}{=} \nu^{\prime} \circ 2 \nu_{6} \circ \eta_{9} \circ \mu_{10}=0,
$$

já que $\nu^{\prime} \circ \nu_{6} \in \pi_{9}^{3}=0$, em $\pi_{19}\left(S^{3} ; 2\right)=\mathbb{Z} / 2\left[\mu_{3} \circ \sigma_{12}\right] \oplus \mathbb{Z} / 2\left[\eta_{3} \circ \bar{\epsilon}_{4}\right]$. Temos assim, $\operatorname{Imev} v_{4}\left(\left[f_{\left[\nu^{\prime} \circ \eta_{6} \circ \mu_{7}\right]}\right]\right)=\mathbb{Z}$. Segue então que

$$
\pi_{2}\left(\mathcal{G}_{\left[\nu^{\prime} \circ \eta_{6} \circ \mu_{7}\right]}\right) \approx \pi_{2}\left(\mathcal{G}_{c_{0}}\right) \approx \pi_{19}\left(S^{3}\right)
$$

Caso $n=18$.

$$
\begin{gathered}
\partial \beta_{f} \in \pi_{17}\left(S^{3}\right) \approx \mathbb{Z} / 30, \\
\partial \beta_{f}(2) \in \pi_{17}\left(S^{3} ; 2\right)=\mathbb{Z} / 2\left[\epsilon_{3} \circ \nu_{11}^{2}\right] .
\end{gathered}
$$

Então temos 30 fibrados. Pela Proposição 4.1 .9 (com $k=4)$ precisamos considerar apenas

$$
\nu^{\prime} \circ \Sigma^{3}\left(\epsilon_{3} \circ \nu_{11}^{2}\right) \stackrel{(16)}{=} \nu^{\prime} \circ \Sigma^{3}\left(\nu^{\prime} \circ \bar{\nu}_{6} \circ \nu_{14}\right) \stackrel{(5)}{=} \nu^{\prime} \circ 2 \nu_{6} \circ \bar{\nu}_{9} \circ \nu_{17}=0,
$$

já que $\nu^{\prime} \circ \nu_{6} \in \pi_{9}^{3}=0$, em $\pi_{20}\left(S^{3} ; 2\right)=\mathbb{Z} / 4\left[\bar{\epsilon}^{\prime}\right] \oplus \mathbb{Z} / 2\left[\bar{\mu}_{3}\right] \oplus \mathbb{Z} / 2\left[\eta_{3} \circ \mu_{4} \circ \sigma_{13}\right]$. Temos $\operatorname{assim} \operatorname{Im} e v_{4}\left(\left[f_{\left[\epsilon_{3} \circ \nu_{11}^{2}\right]}\right]\right)=\mathbb{Z}$. Segue então que

$$
\pi_{2}\left(\mathcal{G}_{\left[\epsilon_{\left.\epsilon_{3} \circ \nu_{11}^{2}\right]}\right.}\right) \approx \pi_{2}\left(\mathcal{G}_{c_{0}}\right) \approx \pi_{20}\left(S^{3}\right)
$$

Caso $n=23$.

$$
\begin{gathered}
\partial \beta_{f} \in \pi_{22}\left(S^{3}\right) \approx \mathbb{Z} / 132 \oplus \mathbb{Z} / 2, \\
\partial \beta_{f}(2) \in \pi_{22}\left(S^{3} ; 2\right)=\mathbb{Z} / 4\left[\bar{\mu}^{\prime}\right] \oplus \mathbb{Z} / 2\left[\nu^{\prime} \circ \mu_{6} \circ \sigma_{15}\right] .
\end{gathered}
$$

Então temos 264 fibrados. Pela Proposição 4.1 .9 (com $k=4)$ precisamos considerar apenas

$$
\nu^{\prime} \circ \Sigma^{3}\left(\bar{\mu}^{\prime}\right) \stackrel{(22)}{=} \nu^{\prime} \circ 2 \bar{\zeta}_{6}=0
$$


pois $\nu^{\prime} \circ \bar{\zeta}_{6} \in \pi_{25}\left(S^{3} ; 2\right) \approx \mathbb{Z} / 2 \mathrm{e}$

$$
\nu^{\prime} \circ \Sigma^{3}\left(\nu^{\prime} \circ \mu_{6} \circ \sigma_{15}\right) \stackrel{(5)}{=} \nu^{\prime} \circ 2 \nu_{6} \circ \mu_{9} \circ \sigma_{18}=0,
$$

já que $\nu^{\prime} \circ \nu_{6} \in \pi_{9}^{3}=0$, em $\pi_{25}\left(S^{3} ; 2\right)=\mathbb{Z} / 2\left[\epsilon_{3} \circ \kappa_{11}\right]$. Então temos $\operatorname{Im} e v_{4}\left(\left[f_{\left[\nu^{\prime} \circ \mu_{6} \circ \sigma_{15}\right]}\right]\right)=$ $\operatorname{Im} e v_{4}\left(\left[f_{\left[\bar{\mu}^{\prime}\right]}\right]\right)=\mathbb{Z}$. Segue então que

$$
\pi_{2}\left(\mathcal{G}_{f_{\left[\nu^{\prime} \circ \mu_{6} \circ \sigma_{15}\right]}}\right) \approx \pi_{2}\left(\mathcal{G}_{f_{\left[\bar{\mu}^{\prime}\right]}}\right) \approx \pi_{2}\left(\mathcal{G}_{c_{0}}\right) \approx \pi_{25}\left(S^{3}\right)
$$

Caso $n=24$.

$$
\begin{gathered}
\partial \beta_{f} \in \pi_{23}\left(S^{3}\right) \approx \mathbb{Z} / 2 \oplus \mathbb{Z} / 2 \\
\partial \beta_{f}(2) \in \pi_{23}\left(S^{3} ; 2\right)=\mathbb{Z} / 2\left[\nu^{\prime} \circ \bar{\mu}_{6}\right] \oplus \mathbb{Z} / 2\left[\nu^{\prime} \circ \eta_{6} \circ \mu_{7} \circ \sigma_{16}\right] .
\end{gathered}
$$

Então temos 4 fibrados. Pela Proposição 4.1.9 precisamos considerar apenas

$$
\nu^{\prime} \circ \Sigma^{3}\left(\nu^{\prime} \circ \bar{\mu}_{6}\right) \stackrel{(5)}{=} \nu^{\prime} \circ 2 \nu_{6} \circ \bar{\mu}_{9}=0
$$

e

$$
\nu^{\prime} \circ \Sigma^{3}\left(\nu^{\prime} \circ \eta_{6} \circ \mu_{7} \circ \sigma_{16}\right) \stackrel{(5)}{=} \nu^{\prime} \circ 2 \nu_{6} \circ \eta_{9} \circ \mu_{10} \circ \sigma_{19}=0,
$$

já que $\nu^{\prime} \circ \nu_{6} \in \pi_{9}^{3}=0$, em $\pi_{26}\left(S^{3} ; 2\right)=\mathbb{Z} / 4[\bar{\alpha}]$. Então temos $\operatorname{Im} e v_{4}\left(\left[f_{\left[\nu^{\prime} \circ \bar{\mu}_{6}\right]}\right]\right)=$ $\operatorname{Im} e v_{4}\left(\left[f_{\left[\nu^{\prime} \circ \eta_{6} \circ \mu_{7} \circ \sigma_{16}\right]}\right]\right)=\mathbb{Z}$. Segue então que

$$
\pi_{2}\left(\mathcal{G}_{f_{\left[\nu^{\prime} \circ \bar{\mu}_{6}\right]}}\right) \approx \pi_{2}\left(\mathcal{G}_{f_{\left[\nu^{\prime} \circ \eta_{6} \circ \mu_{7} \circ \sigma_{16}\right]}}\right) \approx \pi_{2}\left(\mathcal{G}_{c_{0}}\right) \approx \pi_{26}\left(S^{3}\right) .
$$

Caso $n=25$.

$$
\begin{gathered}
\partial \beta_{f} \in \pi_{24}\left(S^{3}\right) \approx \mathbb{Z} / 2, \\
\partial \beta_{f}(2) \in \pi_{24}\left(S^{3} ; 2\right)=\mathbb{Z} / 2\left[\nu^{\prime} \circ \eta_{6} \circ \bar{\mu}_{7}\right] .
\end{gathered}
$$

Então temos 2 fibrados. Pela Proposição 4.1.9 precisamos considerar apenas

$$
\nu^{\prime} \circ \Sigma^{3}\left(\nu^{\prime} \circ \eta_{6} \circ \bar{\mu}_{7}\right) \stackrel{(5)}{=} \nu^{\prime} \circ 2 \nu_{6} \circ \eta_{9} \circ \bar{\mu}_{10}=0
$$

já que $\nu^{\prime} \circ \nu_{6} \in \pi_{9}^{3}=0$, em $\pi_{27}\left(S^{3} ; 2\right)=\mathbb{Z} / 2\left[\delta_{3}\right] \oplus \mathbb{Z} / 2\left[\bar{\mu}_{3} \circ \sigma_{20}\right] \oplus \mathbb{Z} / 2\left[\epsilon^{\prime} \circ \kappa_{13}\right]$. Então temos $\operatorname{Im} e v_{4}\left(\left[f_{\left[\nu^{\prime} \circ \eta_{6} \circ \bar{\mu}_{7}\right]}\right]\right)=\mathbb{Z}$. Segue então que

$$
\pi_{2}\left(\mathcal{G}_{f_{\left[\nu^{\prime} \circ \eta_{6} \circ \bar{\mu}_{7}\right]}}\right) \approx \pi_{2}\left(\mathcal{G}_{c_{0}}\right) \approx \pi_{27}\left(S^{3}\right) .
$$

Proposição 4.3.2. Para $n=5,6$ e 12 , os dois $S^{3}$-fibrados principais sobre $S^{n}$ têm grupos de gauge com diferentes tipos de homotopia. 
Demonstração: Usamos agora a Proposição 4.1.9 e o Lema 4.1.2. Para esses casos, consideramos apenas os fibrados não triviais, devido ao Lema 4.1.10.

Caso $n=5$. Como

$$
\beta_{f} \in \pi_{5}\left(B S^{3}\right)=\mathbb{Z} / 2\left[j_{*}\left(\eta_{4}\right)\right] \approx \pi_{4}\left(S^{3}\right)=\mathbb{Z} / 2\left[\eta_{3}\right]
$$

considere

$$
\partial \beta_{f} \in \pi_{4}\left(S^{3}\right)=\mathbb{Z} / 2\left[\eta_{3}\right]
$$

Temos então 2 fibrados $f_{0}=c_{0}$ e $\partial\left[f_{1}\right]=\eta_{3}$. Pela Proposição $4.1 .9(\operatorname{com} k=4)$ precisamos considerar apenas

$$
\nu^{\prime} \circ \Sigma^{3} \eta_{3}=\nu^{\prime} \circ \eta_{6}
$$

em $\pi_{7}\left(S^{3}\right)=\mathbb{Z} / 2\left[\nu^{\prime} \circ \eta_{6}\right]$ (todos os grupos são de ordem 2). Temos, precisamente, um dos geradores e como ele tem ordem $2, \operatorname{Imev}_{4}\left(\left[f_{1}\right]\right)=2 \mathbb{Z}, \operatorname{Im}_{4}=\mathbb{Z} / \operatorname{Imev}_{4}\left(\left[f_{1}\right]\right)=\mathbb{Z} / 2 \mathbb{Z}$ e $\operatorname{assim} \pi_{2}\left(\mathcal{G}_{f_{1}}\right) \approx \pi_{7}\left(S^{3}\right) /(\mathbb{Z} / 2) \approx(\mathbb{Z} / 2) /(\mathbb{Z} / 2) \approx 0$.

Caso $n=6$.

$$
\partial \beta_{f} \in \pi_{5}\left(S^{3}\right)=\mathbb{Z} / 2\left[\eta_{3}^{2}\right] .
$$

Temos 2 fibrados $f_{0}=c_{0}$ e $\partial\left[f_{1}\right]=\eta_{3}^{2}$. Pela Proposição $4.1 .9(\operatorname{com} k=4)$ precisamos considerar apenas

$$
\nu^{\prime} \circ \Sigma^{3} \eta_{3}^{2}=\nu^{\prime} \circ \eta_{6} \circ \eta_{7}
$$

em $\pi_{8}\left(S^{3}\right)=\mathbb{Z} / 2\left[\nu^{\prime} \circ \eta_{6}^{2}\right]$. Então temos um dos geradores e, como ele tem ordem 2 , $\operatorname{Im} e v_{4}\left(\left[f_{1}\right]\right)=2 \mathbb{Z}, \operatorname{Im} \partial_{4}=\mathbb{Z} / 2 . \operatorname{Logo}, \pi_{2}\left(\mathcal{G}_{f_{1}}\right) \approx \pi_{8}\left(S^{3}\right) /(\mathbb{Z} / 2)=0$.

Caso $n=12$.

$$
\partial \beta_{f} \in \pi_{11}\left(S^{3}\right) \approx \pi_{11}\left(S^{3} ; 2\right)=\mathbb{Z} / 2\left[\epsilon_{3}\right] .
$$

Então temos 2 fibrados $f_{0}=c_{0}$ e $\partial\left[f_{1}\right]=\epsilon_{3}$. Pela Proposição $4.1 .9(\operatorname{com} k=4)$ precisamos considerar apenas

$$
\nu^{\prime} \circ \Sigma^{3} \epsilon_{3}
$$

em $\pi_{14}\left(S^{3} ; 2\right)=\mathbb{Z} / 4\left[\mu^{\prime}\right] \oplus \mathbb{Z} / 2\left[\epsilon_{3} \circ \nu_{11}\right] \oplus \mathbb{Z} / 2\left[\nu^{\prime} \circ \epsilon_{6}\right]$. Então temos, precisamente, um dos geradores e, como ele tem ordem 2 , obtemos $\operatorname{Im} e v_{4}\left(\left[f_{1}\right]\right)=2 \mathbb{Z}, \operatorname{Im} \partial_{4}=\mathbb{Z} / 2$, e assim $\pi_{2}\left(\mathcal{G}_{f_{1}} ; 2\right) \approx \pi_{14}\left(S^{3} ; 2\right) /(\mathbb{Z} / 2)$, o qual não é isomorfo a $\pi_{14}\left(S^{3} ; 2\right)$.

\subsubsection{Os casos $n=14,19,20,22$}

Proposição 4.3.3. Sobre $S^{14}$ existem vinte e quatro $S^{3}$-fibrados principais, classificados pelos elementos $\partial f_{m, n, k}$ de $\pi_{13}\left(S^{3}\right)=\mathbb{Z} / 2\left[x=\eta_{3} \circ \mu_{4}\right] \oplus \mathbb{Z} / 4\left[y=\epsilon^{\prime}\right] \oplus \mathbb{Z} / 3\left[\alpha_{13}\right]$. Os grupos de gauge de $\partial f_{m, n, k}=m x+n y+k \alpha_{13}$ e $\partial f_{m^{\prime}, n^{\prime}, k^{\prime}}^{\prime}=m^{\prime} x+n^{\prime} y+k^{\prime} \alpha_{13}$ têm o mesmo tipo de homotopia se, e somente se, $m=m^{\prime} \bmod 2\left(m, n, k, m^{\prime}, n^{\prime}, k^{\prime} \in \mathbb{Z}\right)$. 
Demonstração: Temos que os fibrados sobre $S^{14}$ são classificados pelas classes

$$
\partial \beta_{f} \in \pi_{13}\left(S^{3}\right) \approx \mathbb{Z} / 2 \oplus \mathbb{Z} / 12
$$

Então, pela Tabela 1 do início do capítulo, temos vinte e quatro fibrados sobre $S^{14}$ (número de fibrados dado pela ordem do grupo), e assim teremos no máximo vinte e quatro diferentes tipos de homotopia para os grupos de gauge. Como precisamos calcular inicialmente Im $e v_{4}$ para posteriormente calcularmos o $\pi_{2}$ do grupo de gauge (Corolário 4.1.3), utilizamos a Proposição 4.1 .9 e por ela vemos que basta considerarmos a 2-componente de $\pi_{13}\left(S^{3}\right) \approx \mathbb{Z} / 2 \oplus \mathbb{Z} / 12$, ou seja, o grupo

$$
\partial \beta_{f}(2) \in \pi_{13}\left(S^{3} ; 2\right)=\mathbb{Z} / 2\left[\eta_{3} \circ \mu_{4}\right] \oplus \mathbb{Z} / 4\left[\epsilon^{\prime}\right] .
$$

Assim, precisamos encontrar para quais valores de $m \in \mathbb{Z}$ as igualdades

$$
m \Sigma \nu^{\prime} \circ \Sigma^{4} \epsilon^{\prime}=0
$$

e

$$
m \Sigma \nu^{\prime} \circ \Sigma^{4}\left(\eta_{3} \circ \mu_{4}\right)=0
$$

são verdadeiras. Mas, observemos que

$$
\nu^{\prime} \circ \Sigma^{3} \epsilon^{\prime} \stackrel{(14)}{=} \nu^{\prime} \circ \Sigma\left(\nu_{5} \circ \sigma_{8}\right)=\nu^{\prime} \circ \nu_{6} \circ \sigma_{9}=0
$$

já que, por [46] pág. 51, o elemento $\nu^{\prime} \circ \nu_{6}$ tem ordem 4 e pertence a $\pi_{9}\left(S^{3}\right)$, o qual não possui elementos de ordem par (ou seja, $\pi_{9}\left(S^{3} ; 2\right)=\pi_{9}^{3}=0$ ) e

$$
\nu^{\prime} \circ \Sigma^{3}\left(\eta_{3} \circ \mu_{4}\right)=\nu^{\prime} \circ \eta_{6} \circ \mu_{7},
$$

em $\pi_{16}\left(S^{3} ; 2\right)=\mathbb{Z} / 2\left[\nu^{\prime} \circ \eta_{6} \circ \mu_{7}\right]$. Então temos, precisamente, um dos geradores e, conseqüentemente, $\operatorname{Im} e v_{4}\left(\left[f_{0, n, k}\right]\right)=\mathbb{Z}$ e $\operatorname{Im} e v_{4}\left(\left[f_{1, n, k}\right]\right)=2 \mathbb{Z}$.

Segue então que

$$
\pi_{2}\left(\mathcal{G}_{f_{0, n, k}}\right) \approx \pi_{16}\left(S^{3}\right) \approx \pi_{2}\left(\mathcal{G}_{c_{0}}\right),
$$

sendo o último isomorfismo dado pelo Lema 4.1 .10 e temos, pelo Lema 4.2.7, que $\mathcal{G}_{f_{0, n, k}} \sim$ $\mathcal{G}_{c_{0}} \mathrm{e}$

$$
\pi_{2}\left(\mathcal{G}_{f_{1, n, k}}\right) \approx \pi_{16}\left(S^{3}\right) / \mathbb{Z} / 2
$$

Proposição 4.3.4. Sobre $S^{19}$ existem trinta $S^{3}$-fibrados principais, classificados pelos elementos $\partial f_{m, n}$ de $\pi_{18}\left(S^{3}\right)=\mathbb{Z} / 2\left[x=\bar{\epsilon}_{3}\right] \oplus \mathbb{Z} / 15\left[\alpha_{18}\right]$. Os grupos de gauge de $f_{m, n}=$ $m x+n \alpha_{18}$ e $\partial f_{m^{\prime}, n^{\prime}}^{\prime}=m^{\prime} x+n^{\prime} \alpha_{18}$ têm o mesmo tipo de homotopia se, e somente se, $m=m^{\prime} \bmod 2\left(m, n, m^{\prime}, n^{\prime} \in \mathbb{Z}\right)$. 


\section{Demonstração:}

$$
\begin{gathered}
\partial \beta_{f} \in \pi_{18}\left(S^{3}\right) \approx \mathbb{Z} / 30, \\
\partial \beta_{f}(2) \in \pi_{18}\left(S^{3} ; 2\right)=\mathbb{Z} / 2\left[\bar{\epsilon}_{3}\right] .
\end{gathered}
$$

Então temos trinta fibrados. Pela Proposição 4.1 .9 (com $k=4)$ precisamos considerar apenas

$$
\nu^{\prime} \circ \Sigma^{3}\left(\bar{\epsilon}_{3}\right)=\nu^{\prime} \circ \bar{\epsilon}_{6}
$$

em $\pi_{21}\left(S^{3} ; 2\right)=\mathbb{Z} / 4\left[\mu^{\prime} \circ \sigma_{14}\right] \oplus \mathbb{Z} / 2\left[\nu^{\prime} \circ \bar{\epsilon}_{6}\right] \oplus \mathbb{Z} / 2\left[\eta_{3} \circ \bar{\mu}_{4}\right]$. Então temos precisamente um dos geradores e, como ele tem ordem 2 , segue $\operatorname{Im} e v_{4}\left(\left[f_{1, n}\right]\right)=2 \mathbb{Z}$. Segue então que

$$
\pi_{2}\left(\mathcal{G}_{f_{1, n}}\right) \approx \pi_{21}\left(S^{3}\right) / \mathbb{Z} / 2
$$

e

$$
\pi_{2}\left(\mathcal{G}_{c_{0}}\right) \approx \pi_{21}\left(S^{3}\right)
$$

Proposição 4.3.5. Sobre $S^{20}$ existem doze $S^{3}$-fibrados principais, classificados pelos elementos $\partial f_{m, n, k}$ de $\pi_{19}\left(S^{3}\right)=\mathbb{Z} / 2\left[x=\eta_{3} \circ \bar{\epsilon}_{4}\right] \oplus \mathbb{Z} / 2\left[y=\mu_{3} \circ \sigma_{12}\right] \oplus \mathbb{Z} / 3\left[\alpha_{19}\right]$. Os grupos de gauge de $f_{m, n, k}=m x+n y+k \alpha_{19}$ e $f_{m^{\prime}, n^{\prime}, k^{\prime}}^{\prime}=m^{\prime} x+n^{\prime} y+k^{\prime} \alpha_{19}$ têm o mesmo tipo de homotopia se, e somente se, $n=n^{\prime} \bmod 2\left(m, n, k, m^{\prime}, n^{\prime}, k^{\prime} \in \mathbb{Z}\right)$.

\section{Demonstração:}

$$
\begin{gathered}
\partial \beta_{f} \in \pi_{19}\left(S^{3}\right) \approx \mathbb{Z} / 6 \oplus \mathbb{Z} / 2, \\
\partial \beta_{f}(2) \in \pi_{19}\left(S^{3} ; 2\right)=\mathbb{Z} / 2\left[\mu_{3} \circ \sigma_{12}\right] \oplus \mathbb{Z} / 2\left[\eta_{3} \circ \bar{\epsilon}_{4}\right] .
\end{gathered}
$$

Então temos doze fibrados. Pela Proposição 4.1 .9 (com $k=4)$ precisamos considerar apenas

$$
\nu^{\prime} \circ \Sigma^{3}\left(\mu_{3} \circ \sigma_{12}\right)=\nu^{\prime} \circ \mu_{6} \circ \sigma_{15}
$$

e

$$
\nu^{\prime} \circ \Sigma^{3}\left(\eta_{3} \circ \bar{\epsilon}_{4}\right)=\nu^{\prime} \circ \eta_{6} \circ \bar{\epsilon}_{7} \stackrel{(23)}{=} \nu^{\prime} \circ \nu_{6} \circ \sigma_{9} \circ \nu_{16}^{2}=0
$$

já que $\nu^{\prime} \circ \nu_{6} \in \pi_{9}^{3}=0$, em $\pi_{22}\left(S^{3} ; 2\right)=\mathbb{Z} / 4\left[\bar{\mu}^{\prime}\right] \oplus \mathbb{Z} / 2\left[\nu^{\prime} \circ \mu_{6} \circ \sigma_{15}\right]$. Então temos, precisamente, um dos geradores e como ele tem ordem 2 , segue $\operatorname{Im} e v_{4}\left(\left[f_{1, n, k}\right]\right)=2 \mathbb{Z}$ e $\operatorname{Im} e v_{4}\left(\left[f_{0, n, k}\right]\right)=\mathbb{Z}$. Segue então que

$$
\pi_{2}\left(\mathcal{G}_{f_{1, n, k}}\right) \approx \pi_{22}\left(S^{3}\right) / \mathbb{Z} / 2
$$

e

$$
\pi_{2}\left(\mathcal{G}_{f_{0, n, k}}\right) \approx \pi_{2}\left(\mathcal{G}_{c_{0}}\right) \approx \pi_{22}\left(S^{3}\right)
$$


Proposição 4.3.6. Sobre $S^{22}$ existem quarenta e oito $S^{3}$-fibrados principais, classificados pelos elementos $\partial f_{m, n, l, k}$ de $\pi_{21}\left(S^{3}\right)=\mathbb{Z} / 2\left[x=\nu^{\prime} \circ \bar{\epsilon}_{6}\right] \oplus \mathbb{Z} / 2\left[y=\eta_{3} \circ \bar{\mu}_{4}\right] \oplus \mathbb{Z} / 4[z=$ $\left.\mu^{\prime} \circ \sigma_{14}\right] \oplus \mathbb{Z} / 3\left[\alpha_{21}\right]$. Os grupos de gauge de $\partial f_{m, n, l, k}=m x+n y+l z+k \alpha_{21}$ e $\partial f_{m^{\prime}, n^{\prime}, l^{\prime}, k^{\prime}}^{\prime}=$ $m^{\prime} x+n^{\prime} y+l^{\prime} z+k^{\prime} \alpha_{21}$ têm o mesmo tipo de homotopia se, e somente se, $n=n^{\prime} \bmod 2$ $\left(m, n, l, k, m^{\prime}, n^{\prime}, l^{\prime}, k^{\prime} \in \mathbb{Z}\right)$.

\section{Demonstração:}

$$
\begin{gathered}
\partial \beta_{f} \in \pi_{21}\left(S^{3}\right) \approx \mathbb{Z} / 12 \oplus \mathbb{Z} / 2 \oplus \mathbb{Z} / 2 \\
\partial \beta_{f}(2) \in \pi_{21}\left(S^{3} ; 2\right)=\mathbb{Z} / 4\left[\mu^{\prime} \circ \sigma_{14}\right] \oplus \mathbb{Z} / 2\left[\nu^{\prime} \circ \bar{\epsilon}_{6}\right] \oplus \mathbb{Z} / 2\left[\eta_{3} \circ \bar{\mu}_{4}\right] .
\end{gathered}
$$

Então temos quarenta e oito fibrados. Pela Proposição 4.1 .9 (com $k=4)$ precisamos considerar apenas

$$
\nu^{\prime} \circ \Sigma^{3}\left(\mu^{\prime} \circ \sigma_{14}\right) \stackrel{(17)}{=} \nu^{\prime} \circ 2 \zeta_{6} \circ \sigma_{17}=0
$$

pois $\nu^{\prime} \circ \zeta_{6} \circ \sigma_{17} \in \pi_{24}\left(S^{3} ; 2\right) \approx \mathbb{Z} / 2$ e

$$
\nu^{\prime} \circ \Sigma^{3}\left(\nu^{\prime} \circ \bar{\epsilon}_{6}\right) \stackrel{(5)}{=} \nu^{\prime} \circ 2 \nu_{6} \circ \bar{\epsilon}_{9}=0,
$$

já que $\nu^{\prime} \circ \nu_{6} \in \pi_{9}^{3}=0$, e

$$
\nu^{\prime} \circ \Sigma^{3}\left(\eta_{3} \circ \bar{\mu}_{4}\right)=\nu^{\prime} \circ \eta_{6} \circ \bar{\mu}_{7}
$$

em $\pi_{24}\left(S^{3} ; 2\right)=\mathbb{Z} / 2\left[\nu^{\prime} \circ \eta_{6} \circ \bar{\mu}_{7}\right]$. Então temos, precisamente, um dos geradores e, como ele tem ordem 2 , segue $\operatorname{Im} e v_{4}\left(\left[f_{n, 1, l, k}\right]\right)=2 \mathbb{Z}$ e $\operatorname{Im} e v_{4}\left(\left[f_{n, 0, l, k}\right]\right)=\mathbb{Z}$. Segue então que

$$
\pi_{2}\left(\mathcal{G}_{f_{n, 1, l, k}}\right) \approx \pi_{23}\left(S^{3}\right) / \mathbb{Z} / 2
$$

e

$$
\pi_{2}\left(\mathcal{G}_{f_{n, 0, l, k}}\right) \approx \pi_{2}\left(\mathcal{G}_{c_{0}}\right) \approx \pi_{24}\left(S^{3}\right)
$$

\subsubsection{O caso de $S^{13}$}

O caso de $S^{13}$, assim como o de $S^{21}$ que veremos na próxima seção, é um pouco mais delicado, uma vez que não temos informações suficientes sobre os grupos de homotopia de $S^{3}$. Vejamos o porquê, começando com o seguinte Lema:

Lema 4.3.7. Sobre $S^{13}$ existem quatro $S^{3}$-fibrados principais, sendo o tipo de homotopia do grupo de gauge do fibrado trivial diferente do tipo de homotopia dos grupos de gauge dos demais fibrados.

Demonstração: Do fato que

$$
\partial \beta_{f} \in \pi_{12}\left(S^{3}\right)=\mathbb{Z} / 2\left[\mu_{3}\right] \oplus \mathbb{Z} / 2\left[\eta_{3} \circ \epsilon_{4}\right]
$$


temos então quatro fibrados sobre $S^{13},\left[f_{(0,0)}\right]=\left[c_{0}\right], \partial\left[f_{(1,0)}\right]=\mu_{3}, \partial\left[f_{(0,1)}\right]=\eta_{3} \circ \epsilon_{4}$, $\partial\left[f_{(1,1)}\right]=\mu_{3}+\eta_{3} \circ \epsilon_{4}$. Pela Proposição $4.1 .9(\operatorname{com} k=4)$ precisamos considerar apenas

$$
\nu^{\prime} \circ \Sigma^{3} \mu_{3}=\nu^{\prime} \circ \mu_{6}
$$

$\mathrm{e}$

$$
\nu^{\prime} \circ \Sigma^{3}\left(\eta_{3} \circ \epsilon_{4}\right)=\nu^{\prime} \circ \eta_{6} \circ \epsilon_{7},
$$

em $\pi_{15}\left(S^{3} ; 2\right)=\mathbb{Z} / 2\left[\nu^{\prime} \circ \mu_{6}\right] \oplus \mathbb{Z} / 2\left[\nu^{\prime} \circ \eta_{6} \circ \epsilon_{7}\right]$. Então temos, precisamente, os geradores de $\pi_{15}\left(S^{3} ; 2\right)$ e, conseqüentemente, $\operatorname{Im} e v_{4}\left(\left[f_{(i, j) \neq(0,0)}\right]\right)=2 \mathbb{Z}, \operatorname{Im} \partial_{4}=\mathbb{Z} / 2$ e $\pi_{2}\left(\mathcal{G}_{f_{(i, j) \neq(0,0)}}\right) \approx \mathbb{Z} / 2$ enquanto $\pi_{2}\left(\mathcal{G}_{f_{(0,0)}}\right) \approx \pi_{15}\left(S^{3}\right)=\pi_{15}\left(S^{3} ; 2\right) \approx \mathbb{Z} / 2 \oplus \mathbb{Z} / 2$.

Esse resultado ainda não nos dá informação suficiente para sabermos quantos tipos de homotopias diferentes têm os grupos de gauge dos fibrados sobre $S^{3}$, pois nada podemos dizer sobre os três fibrados não triviais $\left(\partial\left[f_{(1,0)}\right]=\mu_{3}, \partial\left[f_{(0,1)}\right]=\eta_{3} \circ \epsilon_{4}, \partial\left[f_{(1,1)}\right]=\right.$ $\left.\mu_{3}+\eta_{3} \circ \epsilon_{4}\right)$. Para obtermos informações sobre eles, continuamos calculando os grupos de homotopia dos grupos de gauge desses fibrados e obtemos os seguintes lemas:

Lema 4.3.8. Para $n=3,4$ temos $\pi_{n}\left(\mathcal{G}_{f_{(0,1)}}\right) \not \approx \pi_{n}\left(\mathcal{G}_{f_{(1,0)}}\right) \approx \pi_{n}\left(\mathcal{G}_{f_{(1,1)}}\right)$, e com isso o tipo de homotopia do grupo de gauge do fibrado $\partial\left[f_{(0,1)}\right]=\eta_{3} \circ \epsilon_{4}$ é diferente do demais.

Demonstração: Caso $\pi_{3}\left(\mathcal{G}_{f}\right)$. Consideremos a seqüência do Lema $4.1 .2 \operatorname{com} k=4$ :

$$
\begin{gathered}
\pi_{4}\left(S^{3}\right) \underset{\partial_{5}}{\longrightarrow} \pi_{16}\left(S^{3}\right) \underset{ }{\longrightarrow} \pi_{3}\left(\mathcal{G}_{f}\right) \underset{e v_{4}}{\longrightarrow} \pi_{3}\left(S^{3}\right) \underset{\partial_{4}}{\longrightarrow} \pi_{15}\left(S^{3}\right), \\
\mathbb{Z} / 2 \underset{\partial_{5}}{\longrightarrow} \mathbb{Z} / 6 \underset{\phi}{\longrightarrow} \pi_{3}\left(\mathcal{G}_{f}\right) \underset{e v_{4}}{\longrightarrow} \mathbb{Z} \underset{\partial_{4}}{\longrightarrow} \mathbb{Z} \oplus \mathbb{Z} / 2 .
\end{gathered}
$$

Temos que

$$
\operatorname{Nuc} \phi=\operatorname{Im} \partial_{5}=0 \text { ou } \mathbb{Z} / 2 \text {. }
$$

Assim,

$$
\operatorname{Im} \phi=(\mathbb{Z} / 6) / \operatorname{Nuc} \phi=\mathbb{Z} / 6 \text { ou } \mathbb{Z} / 3 \text {. }
$$

Agora, sendo um grupo abeliano finitamente gerado $A=\pi_{3}\left(\mathcal{G}_{f}\right) \approx \mathbb{Z}^{m} \oplus T$, no qual $T$ é o subgrupo de torção. Seja $f \neq c_{0}$. Então sabemos, pelos cálculos do lema anterior, que

$$
\operatorname{Im} e v_{4}=2 \mathbb{Z} \text { e } \operatorname{Im} \partial_{4}=\mathbb{Z} / 2
$$

Logo,

$$
2 \mathbb{Z}=\operatorname{Im} e v_{4}=A / \operatorname{Nuc} e v_{4}=\left(\mathbb{Z}^{m} \oplus T\right) / \operatorname{Nuc} e v_{4}=\left(\mathbb{Z}^{m} \oplus T\right) / \operatorname{Im} \phi
$$

implica que $m=1$ e $T=\mathbb{Z} / 3$ ou $T=\mathbb{Z} / 6$.

Para decidir qual é o caso, considere que

$$
\operatorname{Im} \phi=(\mathbb{Z} / 6) / \mathrm{Nuc} \phi,
$$




$$
\operatorname{Nuc} \phi=\operatorname{Im} \partial_{5}=0 \text { ou } \mathbb{Z} / 2
$$

e

$$
\operatorname{Im} \partial_{5}=(\mathbb{Z} / 2) / \operatorname{Im} e v_{5} .
$$

Precisamos calcular Im $e v_{5}$. Pela Proposição 4.1 .8 (com $\left.k=5\right)$, precisamos resolver a equação

$$
\nu^{\prime} \circ \Sigma^{3} \partial \beta_{f}(2) \circ \Sigma^{12} \partial \omega(2)=0
$$

em $\pi_{16}\left(S^{3} ; 2\right)=\mathbb{Z} / 2\left[\nu^{\prime} \circ \eta_{6} \circ \mu_{7}\right]$, com

$$
\partial \beta_{f}(2) \in \pi_{12}\left(S^{3} ; 2\right)=\mathbb{Z} / 2\left[\eta_{3} \circ \epsilon_{4}\right] \oplus \mathbb{Z} / 2\left[\mu_{3}\right]
$$

e

$$
\partial \omega(2) \in \pi_{4}\left(S^{3} ; 2\right)=\mathbb{Z} / 2\left[\eta_{3}\right] .
$$

Calculemos então $\nu^{\prime} \circ \Sigma^{3} \partial \beta_{f}(2)$ para as duas possibilidades,

$$
\nu^{\prime} \circ \Sigma^{3}\left(\eta_{3} \circ \epsilon_{4}\right)=\nu^{\prime} \circ \eta_{6} \circ \epsilon_{7}
$$

e

$$
\nu^{\prime} \circ \Sigma^{3}\left(\mu_{3}\right)=\nu^{\prime} \circ \mu_{6},
$$

ambos em $\pi_{15}\left(S^{3} ; 2\right)=\mathbb{Z} / 2\left[\nu^{\prime} \circ \eta_{6} \circ \epsilon_{7}\right] \oplus \mathbb{Z} / 2\left[\nu^{\prime} \circ \mu_{6}\right]$.

Compondo com o possível

$$
\Sigma^{12} \partial \omega=(n \bmod 2) \eta_{15}
$$

obtemos elementos de $\pi_{16}\left(S^{3} ; 2\right)$.

O primeiro é:

$$
\begin{gathered}
\nu^{\prime} \circ \Sigma^{3}\left(\eta_{3} \circ \epsilon_{4}\right) \circ \eta_{15}=\nu^{\prime} \circ \eta_{6} \circ \epsilon_{7} \circ \eta_{15}=\nu^{\prime} \circ \eta_{6} \circ \eta_{7} \circ \epsilon_{8} \\
=\nu^{\prime} \circ 4\left(\nu_{6} \circ \sigma_{9}\right)=4 \nu^{\prime} \circ \nu_{6} \circ \sigma_{9}=0,
\end{gathered}
$$

no qual aplicamos a relação (1) do Apêndice do final do capítulo,

$$
\epsilon_{6} \circ \eta_{14}=\Sigma^{3} \epsilon_{3} \circ \Sigma^{3+8} \eta_{3}=\Sigma^{3} \eta_{3} \circ \Sigma^{3+1} \epsilon_{3},
$$

com $\epsilon_{3} \in \pi_{11}\left(S^{3}\right), \eta_{3} \in \pi_{4}\left(S^{3}\right)$; e por 7.10 de [46] pág. 67

$$
\eta_{n}^{2} \circ \epsilon_{n+2}=4\left(\nu_{n} \circ \sigma_{n+3}\right),
$$

para $n \geq 5$.

O segundo é:

$$
\nu^{\prime} \circ \Sigma^{3}\left(\mu_{3}\right) \circ \eta_{15}=\nu^{\prime} \circ \mu_{6} \circ \eta_{15}=\nu^{\prime} \circ \eta_{6} \circ \mu_{7},
$$

que é exatamente o gerador.

Recordemos que denotamos as três aplicações não triviais por $f_{i, j}, 0 \leq i, j \leq 1$. Logo,

$$
\operatorname{Im} e v_{5}\left(\left[f_{1,0}\right]\right)=0,
$$




$$
\begin{gathered}
\operatorname{Im} e v_{5}\left(\left[f_{0,1}\right]\right)=\pi_{4}\left(S^{3} ; 2\right)=\mathbb{Z} / 2, \\
\operatorname{Im} e v_{5}\left(\left[f_{1,1}\right]\right)=0
\end{gathered}
$$

Caso $\pi_{4}\left(\mathcal{G}_{f}\right)$. Consideremos a seqüência no Lema 4.1.2 com $k=5$ :

$$
\begin{gathered}
\pi_{5}\left(S^{3}\right) \underset{\partial_{6}}{\longrightarrow} \pi_{17}\left(S^{3}\right) \longrightarrow \pi_{4}\left(\mathcal{G}_{f}\right) \underset{e v_{5}}{\longrightarrow} \pi_{4}\left(S^{3}\right) \underset{\partial_{5}}{\longrightarrow} \pi_{16}\left(S^{3}\right), \\
\mathbb{Z} / 2 \underset{\partial_{6}}{\longrightarrow} \mathbb{Z} / 30 \underset{\phi}{\longrightarrow} \pi_{4}\left(\mathcal{G}_{f}\right) \underset{\text { ev }}{\longrightarrow} \mathbb{Z} / 2 \underset{\partial_{5}}{\longrightarrow} \mathbb{Z} / 6
\end{gathered}
$$

É claro que

$$
\operatorname{Nuc} \phi=\operatorname{Im} \partial_{6}=0 \text { ou } \mathbb{Z} / 2 \text {. }
$$

Assim

$$
\operatorname{Im} \phi=(\mathbb{Z} / 30) / \operatorname{Nuc} \phi=\mathbb{Z} / 30 \text { ou } \mathbb{Z} / 15 \text {. }
$$

Agora, como grupo abeliano finitamente gerado, $A=\pi_{4}\left(\mathcal{G}_{f}\right) \approx \mathbb{Z}^{m} \oplus T$, onde $T$ é o subgrupo de torção. Seja $f \neq c_{0}$ (recordemos que $\partial\left[f_{1,0}\right]=\mu_{3}, \partial\left[f_{0,1}\right]=\eta_{3} \circ \epsilon_{4}, \partial\left[f_{1,1}\right]=$ $\left.\mu_{3} \oplus \eta_{3} \circ \epsilon_{4}\right)$. Dos cálculos feitos na Seção 4.3.3, temos que

$$
\begin{gathered}
\operatorname{Im} e v_{5}\left(\left[f_{1,0}\right]\right)=0, \\
\operatorname{Im} e v_{5}\left(\left[f_{0,1}\right]\right)=\mathbb{Z} / 2, \\
\operatorname{Im} e v_{5}\left(\left[f_{1,1}\right]\right)=0,
\end{gathered}
$$

Então

$$
0=\operatorname{Imev}_{5}\left(\left[f_{1,0}\right]\right)=\operatorname{Imev}_{5}\left(\left[f_{1,1}\right]\right)=A / \operatorname{Nucev}_{5}=\left(\mathbb{Z}^{m} \oplus T\right) / \operatorname{Nucev}_{5}=\left(\mathbb{Z}^{m} \oplus T\right) / \operatorname{Im} \phi,
$$

implica que $m=0$ e $T=\mathbb{Z} / 30$ ou $T=\mathbb{Z} / 15$, e

$$
\mathbb{Z} / 2=\operatorname{Im} e v_{5}\left(\left[f_{0,1}\right]\right)=A / \operatorname{Nuc} e v_{5}=\left(\mathbb{Z}^{m} \oplus T\right) / \operatorname{Nuc} e v_{5}=\left(\mathbb{Z}^{m} \oplus T\right) / \operatorname{Im} \phi,
$$

implica que $m=0$ e $T=\mathbb{Z} / 60$ ou $T=\mathbb{Z} / 30 \oplus \mathbb{Z} / 2$ ou $T=\mathbb{Z} / 30$.

Para decidirmos qual é o caso, consideremos

$$
\operatorname{Im} \phi=(\mathbb{Z} / 30) / \text { Nuc } \phi,
$$

$\operatorname{Nuc} \phi=\operatorname{Im} \partial_{6}=0$ ou $\mathbb{Z} / 2$

e

$$
\operatorname{Im} \partial_{6}=(\mathbb{Z} / 2) / \operatorname{Im} e v_{6} .
$$

Precisamos calcular Im $e v_{6}$. Pela Proposição 4.1 .8 (com $\left.k=6\right)$, precisamos resolver a equação

$$
\nu^{\prime} \circ \Sigma^{3} \partial \beta_{f}(2) \circ \Sigma^{12} \partial \omega(2)=0
$$


em $\pi_{17}\left(S^{3} ; 2\right)=\mathbb{Z} / 2\left[\epsilon_{3} \circ \nu_{11}^{2}\right]$, sendo

$$
\partial \beta_{f}(2) \in \pi_{12}\left(S^{3} ; 2\right)=\mathbb{Z} / 2\left[\eta_{3} \circ \epsilon_{4}\right] \oplus \mathbb{Z} / 2\left[\mu_{3}\right]
$$

e

$$
\partial \omega(2) \in \pi_{5}\left(S^{3} ; 2\right)=\mathbb{Z} / 2\left[\eta_{3}^{2}\right] .
$$

Calculando $\nu^{\prime} \circ \Sigma^{3} \partial \beta_{f}(2)$ para as duas possibilidades,

$$
\nu^{\prime} \circ \Sigma^{3}\left(\eta_{3} \circ \epsilon_{4}\right)=\nu^{\prime} \circ \eta_{6} \circ \epsilon_{7}
$$

e

$$
\nu^{\prime} \circ \Sigma^{3}\left(\mu_{3}\right)=\nu^{\prime} \circ \mu_{6},
$$

ambos em $\pi_{15}\left(S^{3} ; 2\right)=\mathbb{Z} / 2\left[\nu^{\prime} \circ \eta_{6} \circ \epsilon_{7}\right] \oplus \mathbb{Z} / 2\left[\nu^{\prime} \circ \mu_{6}\right]$.

Compondo com o possível

$$
\Sigma^{12} \partial \omega=(n \bmod 2) \eta_{15}^{2}
$$

obtemos elementos de $\pi_{17}\left(S^{3} ; 2\right)$.

O primeiro é:

$$
\begin{gathered}
\nu^{\prime} \circ \Sigma^{3}\left(\eta_{3} \circ \epsilon_{4}\right) \circ \eta_{15}^{2}=\nu^{\prime} \circ \eta_{6} \circ \epsilon_{7} \circ \eta_{15} \circ \eta_{16} \stackrel{(1)}{=} \nu^{\prime} \circ \eta_{6} \circ \eta_{7} \circ \epsilon_{8} \circ \eta_{16} \\
\stackrel{(14)}{=} \nu^{\prime} \circ 4\left(\nu_{6} \circ \sigma_{9}\right) \circ \eta_{16}=4 \nu^{\prime} \circ \nu_{6} \circ \sigma_{9} \circ \eta_{16}=0 .
\end{gathered}
$$

Portanto,

$$
\begin{gathered}
\operatorname{Im} e v_{6}\left(\left[f_{0,1}\right]\right)=\mathbb{Z} / 2, \\
\operatorname{Im} \partial_{6}=0, \\
\operatorname{Im} \phi=\mathbb{Z} / 30, \\
T=\mathbb{Z} / 60 \text { ou } T=\mathbb{Z} / 30 \oplus \mathbb{Z} / 2, \\
\pi_{4}\left(\mathcal{G}_{f}\right) \approx \mathbb{Z} / 60 \text { ou } \mathbb{Z} / 30 \oplus \mathbb{Z} / 2 .
\end{gathered}
$$

O segundo:

$$
\begin{gathered}
\nu^{\prime} \circ \Sigma^{3}\left(\mu_{3}\right) \circ \eta_{15}^{2}=\nu^{\prime} \circ \mu_{6} \circ \eta_{15} \circ \eta_{16} \stackrel{(1)}{=} \nu^{\prime} \circ \eta_{6} \circ \mu_{7} \circ \eta_{16} \\
\stackrel{(1)}{=} \nu^{\prime} \circ \eta_{6} \circ \eta_{7} \circ \mu_{8} \stackrel{(17)}{=} \nu^{\prime} \circ 4 \zeta_{6}=4 \nu^{\prime} \circ \zeta_{6}=0 .
\end{gathered}
$$

Logo,

$$
\begin{gathered}
\operatorname{Im} e v_{6}\left(\left[f_{1,0}\right]\right)=\operatorname{Im} e v_{6}\left(\left[f_{1,1}\right]\right)=\mathbb{Z} / 2, \\
\operatorname{Im} \partial_{6}=0 \\
\operatorname{Im} \phi=\mathbb{Z} / 30 \\
T=\mathbb{Z} / 30 \\
\pi_{4}\left(\mathcal{G}_{f}\right) \approx \mathbb{Z} / 30 .
\end{gathered}
$$


Lema 4.3.9. Para $5 \leq n \leq 9$ temos $\pi_{n}\left(\mathcal{G}_{f_{(0,1)}}\right) \approx \pi_{n}\left(\mathcal{G}_{f_{(1,0)}}\right) \approx \pi_{n}\left(\mathcal{G}_{f_{(1,1)}}\right)$.

Demonstração: Caso $\pi_{5}\left(\mathcal{G}_{f}\right)$. Considerando a seqüência no Lema 4.1.2 (com $\left.k=6\right)$,

$$
\begin{gathered}
\pi_{6}\left(S^{3}\right) \underset{\partial_{7}}{\longrightarrow} \pi_{18}\left(S^{3}\right) \longrightarrow \pi_{5}\left(\mathcal{G}_{f}\right) \underset{e v_{6}}{\longrightarrow} \pi_{5}\left(S^{3}\right) \underset{\partial_{6}}{\longrightarrow} \pi_{17}\left(S^{3}\right), \\
\mathbb{Z} / 12 \underset{\partial_{7}}{\longrightarrow} \mathbb{Z} / 30 \underset{\phi}{\longrightarrow} \pi_{5}\left(\mathcal{G}_{f}\right) \underset{\text { ev }}{\longrightarrow} \mathbb{Z} / 2 \underset{\partial_{6}}{\longrightarrow} \mathbb{Z} / 30 .
\end{gathered}
$$

É claro que

$$
\operatorname{Nuc} \phi=\operatorname{Im} \partial_{7}=0 \text { ou } \mathbb{Z} / 2 \text { ou } \mathbb{Z} / 3 \text { ou } \mathbb{Z} / 6 \text {, }
$$

assim

$$
\operatorname{Im} \phi=(\mathbb{Z} / 30) / \text { Nuc } \phi=\mathbb{Z} / 30 \text { ou } \mathbb{Z} / 15 \text { ou } \mathbb{Z} / 10 \text { ou } \mathbb{Z} / 5 \text {. }
$$

Agora, como um grupo abeliano finitamente gerado, $A=\pi_{5}\left(\mathcal{G}_{f}\right) \approx \mathbb{Z}^{m} \oplus T$, sendo $T$ o subgrupo de torção. Seja $f \neq c_{0}$ (recorde que $\partial\left[f_{1,0}\right]=\mu_{3}, \partial\left[f_{0,1}\right]=\eta_{3} \circ \epsilon_{4}, \partial\left[f_{1,1}\right]=$ $\left.\mu_{3} \oplus \eta_{3} \circ \epsilon_{4}\right)$. Dos cálculos do caso anterior, temos

$$
\operatorname{Im} e v_{6}\left(\left[f_{1,0}\right]\right)=\operatorname{Im} e v_{6}\left(\left[f_{0,1}\right]\right)=\operatorname{Im} e v_{6}\left(\left[f_{1,1}\right]\right)=\mathbb{Z} / 2
$$

Então

$$
\mathbb{Z} / 2=\operatorname{Im} e v_{6}=A / \operatorname{Nuc} e v_{6}=\left(\mathbb{Z}^{m} \oplus T\right) / \operatorname{Nuc} e v_{6}=\left(\mathbb{Z}^{m} \oplus T\right) / \operatorname{Im} \phi
$$

implica que $m=0$ e $T=\mathbb{Z} / 60$ ou $T=\mathbb{Z} / 30 \oplus \mathbb{Z} / 2$ ou $T=\mathbb{Z} / 30$ ou $T=\mathbb{Z} / 10 \oplus \mathbb{Z} / 2$ ou $T=\mathbb{Z} / 20$ ou $T=\mathbb{Z} / 10$.

Para decidir qual é o caso, consideremos que

$$
\operatorname{Im} \phi=(\mathbb{Z} / 30) / N u c \phi,
$$

$$
\operatorname{Nuc} \phi=\operatorname{Im} \partial_{7}=0 \text { ou } \mathbb{Z} / 2 \text { ou } \mathbb{Z} / 3 \text { ou } \mathbb{Z} / 6
$$

e

$$
\operatorname{Im} \partial_{7}=(\mathbb{Z} / 12) / \operatorname{Im} e v_{7}
$$

Precisamos calcular Im $e v_{7}$. Pela Proposição 4.1 .8 (com $k=7$ ), precisamos resolver a equação

$$
\nu^{\prime} \circ \Sigma^{3} \partial \beta_{f}(2) \circ \Sigma^{12} \partial \omega(2)=0
$$

em $\pi_{18}\left(S^{3} ; 2\right)=\mathbb{Z} / 2\left[\bar{\epsilon}_{3}\right]$, com

$$
\partial \beta_{f}(2) \in \pi_{12}\left(S^{3} ; 2\right)=\mathbb{Z} / 2\left[\eta_{3} \circ \epsilon_{4}\right] \oplus \mathbb{Z} / 2\left[\mu_{3}\right]
$$

e

$$
\partial \omega(2) \in \pi_{6}\left(S^{3} ; 2\right)=\mathbb{Z} / 4\left[\nu^{\prime}\right] .
$$

Calculando então $\nu^{\prime} \circ \Sigma^{3} \partial \beta_{f}(2)$ para as duas possibilidades,

$$
\nu^{\prime} \circ \Sigma^{3}\left(\eta_{3} \circ \epsilon_{4}\right)=\nu^{\prime} \circ \eta_{6} \circ \epsilon_{7}
$$


e

$$
\nu^{\prime} \circ \Sigma^{3}\left(\mu_{3}\right)=\nu^{\prime} \circ \mu_{6},
$$

ambos em $\pi_{15}\left(S^{3} ; 2\right)=\mathbb{Z} / 2\left[\nu^{\prime} \circ \eta_{6} \circ \epsilon_{7}\right] \oplus \mathbb{Z} / 2\left[\nu^{\prime} \circ \mu_{6}\right]$.

Compondo com o possível

$$
\Sigma^{12} \partial \omega=(n \bmod 4) \Sigma^{12}\left(\nu^{\prime}\right)
$$

obtemos elementos de $\pi_{18}\left(S^{3} ; 2\right)$.

O primeiro:

$$
\begin{gathered}
\nu^{\prime} \circ \Sigma^{3}\left(\eta_{3} \circ \epsilon_{4}\right) \circ \Sigma^{12}\left(\nu^{\prime}\right) \stackrel{(4)}{=} \nu^{\prime} \circ \eta_{6} \circ \epsilon_{7} \circ \Sigma^{10}\left(2 \Sigma \nu_{4}\right) \\
=2 \nu^{\prime} \circ \eta_{6} \circ \epsilon_{7} \circ \Sigma^{11} \nu_{4} \stackrel{(1)}{=} 2 \nu^{\prime} \circ \eta_{6} \circ \nu_{7} \circ \epsilon_{10} \stackrel{(9)}{=} 0 .
\end{gathered}
$$

Logo,

$$
\begin{gathered}
\operatorname{Im} e v_{7}\left(\left[f_{0,1}\right]\right)=\mathbb{Z} / 12, \\
\operatorname{Im} \partial_{7}=0, \\
\operatorname{Im} \phi=\mathbb{Z} / 30, \\
T=\mathbb{Z} / 60 \text { ou } T=\mathbb{Z} / 30 \oplus \mathbb{Z} / 2, \\
\pi_{5}\left(\mathcal{G}_{f}\right) \approx \mathbb{Z} / 60 \text { ou } \mathbb{Z} / 30 \oplus \mathbb{Z} / 2 .
\end{gathered}
$$

O segundo é:

$$
\nu^{\prime} \circ \Sigma^{3}\left(\mu_{3}\right) \circ \Sigma^{12}\left(\nu^{\prime}\right) \stackrel{(4)}{=} \nu^{\prime} \circ \mu_{6} \circ \Sigma^{10}\left(2 \Sigma \nu_{4}\right)=\nu^{\prime} \circ 2 \mu_{6} \circ \Sigma^{11} \nu_{4} \stackrel{(10)}{=} 0 .
$$

Portanto,

$$
\begin{gathered}
\operatorname{Im} e v_{7}\left(\left[f_{1,0}\right]\right)=\operatorname{Im} e v_{7}\left(\left[f_{1,1}\right]\right)=\mathbb{Z} / 12, \\
\operatorname{Im} \partial_{7}=0, \\
\operatorname{Im} \phi=\mathbb{Z} / 30, \\
T=\mathbb{Z} / 60 \text { ou } T=\mathbb{Z} / 30 \oplus \mathbb{Z} / 2, \\
\pi_{5}\left(\mathcal{G}_{f}\right)=\mathbb{Z} / 60 \text { ou } \mathbb{Z} / 30 \oplus \mathbb{Z} / 2 .
\end{gathered}
$$

Caso $\pi_{6}\left(\mathcal{G}_{f}\right)$. Consideremos a seqüência no Lema 4.1.2 (com $\left.k=7\right)$,

$$
\begin{gathered}
\pi_{7}\left(S^{3}\right) \underset{\partial_{8}}{\longrightarrow} \pi_{19}\left(S^{3}\right) \longrightarrow \pi_{6}\left(\mathcal{G}_{f}\right) \underset{e v_{7}}{\longrightarrow} \pi_{6}\left(S^{3}\right) \underset{\partial_{7}}{\longrightarrow} \pi_{18}\left(S^{3}\right), \\
\mathbb{Z} / 2 \underset{\partial_{8}}{\longrightarrow} \mathbb{Z} / 6 \oplus \mathbb{Z} / 2 \underset{\phi}{\longrightarrow} \pi_{6}\left(\mathcal{G}_{f}\right) \underset{e v_{7}}{\longrightarrow} \mathbb{Z} / 12 \underset{\partial_{7}}{\longrightarrow} \mathbb{Z} / 30 .
\end{gathered}
$$

É claro que

$$
\text { Nuc } \phi=\operatorname{Im} \partial_{8}=0 \text { ou } \mathbb{Z} / 2 \text {. }
$$


Assim

$$
\operatorname{Im} \phi=(\mathbb{Z} / 6 \oplus \mathbb{Z} / 2) / \text { Nuc } \phi=\mathbb{Z} / 6 \oplus \mathbb{Z} / 2 \text { ou } \mathbb{Z} / 6 \text {. }
$$

Agora, como um grupo abeliano finitamente gerado, $A=\pi_{6}\left(\mathcal{G}_{f}\right) \approx \mathbb{Z}^{m} \oplus T$, sendo $T$ o subgrupo de torção. Seja $f \neq c_{0}$ (recordemos que $\partial\left[f_{1,0}\right]=\mu_{3}, \partial\left[f_{0,1}\right]=\eta_{3} \circ \epsilon_{4}, \partial\left[f_{1,1}\right]=$ $\left.\mu_{3} \oplus \eta_{3} \circ \epsilon_{4}\right)$. Dos cáculos feitos no caso anterior, temos

$$
\operatorname{Im} e v_{7}\left(\left[f_{1,0}\right]\right)=\operatorname{Im} e v_{7}\left(\left[f_{0,1}\right]\right)=\operatorname{Im} e v_{7}\left(\left[f_{1,1}\right]\right)=\mathbb{Z} / 12,
$$

Então

$$
\mathbb{Z} / 12=\operatorname{Im} e v_{7}=A / \operatorname{Nuc} e v_{7}=\left(\mathbb{Z}^{m} \oplus T\right) / \operatorname{Nuc} e v_{7}=\left(\mathbb{Z}^{m} \oplus T\right) / \operatorname{Im} \phi,
$$

implica que $m=0$ e $T=\mathbb{Z} / 72 \oplus \mathbb{Z} / 2$ ou $T=\mathbb{Z} / 6 \oplus \mathbb{Z} / 24$ ou $T=\mathbb{Z} / 12 \oplus \mathbb{Z} / 6 \oplus \mathbb{Z} / 2$ ou $T=\mathbb{Z} / 12 \oplus \mathbb{Z} / 6$ ou $T=\mathbb{Z} / 72$.

Para decidirmos qual é o caso, consideremos

$$
\begin{gathered}
\operatorname{Im} \phi=(\mathbb{Z} / 6 \oplus \mathbb{Z} / 2) / \text { Nuc } \phi, \\
\text { Nuc } \phi=\operatorname{Im} \partial_{8}=0 \text { ou } \mathbb{Z} / 2
\end{gathered}
$$

e

$$
\operatorname{Im} \partial_{8}=(\mathbb{Z} / 2) / \operatorname{Im} e v_{8} .
$$

Precisamos calcular Im $e v_{8}$. Pela Proposição 4.1 .8 (com $\left.k=8\right)$, precisamos resolver a equação

$$
\nu^{\prime} \circ \Sigma^{3} \partial \beta_{f}(2) \circ \Sigma^{12} \partial \omega(2)=0
$$

em $\pi_{19}\left(S^{3} ; 2\right)=\mathbb{Z} / 2\left[\mu_{3} \circ \sigma_{12}\right] \oplus \mathbb{Z} / 2\left[\eta_{3} \circ \bar{\epsilon}_{4}\right]$, com

$$
\partial \beta_{f}(2) \in \pi_{12}\left(S^{3} ; 2\right)=\mathbb{Z} / 2\left[\eta_{3} \circ \epsilon_{4}\right] \oplus \mathbb{Z} / 2\left[\mu_{3}\right]
$$

e

$$
\partial \omega(2) \in \pi_{7}\left(S^{3} ; 2\right)=\mathbb{Z} / 2\left[\nu^{\prime} \circ \eta_{6}\right] .
$$

Calculando $\nu^{\prime} \circ \Sigma^{3} \partial \beta_{f}(2)$ para as duas possibilidades

$$
\nu^{\prime} \circ \Sigma^{3}\left(\eta_{3} \circ \epsilon_{4}\right)=\nu^{\prime} \circ \eta_{6} \circ \epsilon_{7}
$$

e

$$
\nu^{\prime} \circ \Sigma^{3}\left(\mu_{3}\right)=\nu^{\prime} \circ \mu_{6},
$$

ambos em $\pi_{15}\left(S^{3} ; 2\right)=\mathbb{Z} / 2\left[\nu^{\prime} \circ \eta_{6} \circ \epsilon_{7}\right] \oplus \mathbb{Z} / 2\left[\nu^{\prime} \circ \mu_{6}\right]$.

Compondo com o possível

$$
\Sigma^{12} \partial \omega=(n \bmod 2) \Sigma^{12}\left(\nu^{\prime}\right) \circ \eta_{18},
$$

obtemos elementos de $\pi_{19}\left(S^{3} ; 2\right)$. 
O primeiro é:

$$
\begin{gathered}
\nu^{\prime} \circ \Sigma^{3}\left(\eta_{3} \circ \epsilon_{4}\right) \circ \Sigma^{12}\left(\nu^{\prime} \circ \eta_{6}\right) \stackrel{(4)}{=} \nu^{\prime} \circ \eta_{6} \circ \epsilon_{7} \circ \Sigma^{10}\left(2 \Sigma \nu_{4}\right) \circ \eta_{18} \\
=2 \nu^{\prime} \circ \eta_{6} \circ \epsilon_{7} \circ \Sigma^{11} \nu_{4} \circ \eta_{18} \stackrel{(1)}{=} 2 \nu^{\prime} \circ \eta_{6} \circ \nu_{7} \circ \epsilon_{10} \circ \eta_{18} \stackrel{(9)}{=} 0 .
\end{gathered}
$$

Portanto,

$$
\begin{gathered}
\operatorname{Im} e v_{8}\left(\left[f_{0,1}\right]\right)=\mathbb{Z} / 2, \\
\operatorname{Im} \partial_{8}=0, \\
\operatorname{Im} \phi=\mathbb{Z} / 6 \oplus \mathbb{Z} / 2, \\
T=\mathbb{Z} / 72 \oplus \mathbb{Z} / 2 \text { ou } T=\mathbb{Z} / 24 \oplus \mathbb{Z} / 6 \text { ou } T=\mathbb{Z} / 12 \oplus \mathbb{Z} / 6 \oplus \mathbb{Z} / 2, \\
\pi_{6}\left(\mathcal{G}_{f}\right) \approx \mathbb{Z} / 72 \oplus \mathbb{Z} / 2 \text { ou } \mathbb{Z} / 24 \oplus \mathbb{Z} / 6 \text { ou } \mathbb{Z} / 12 \oplus \mathbb{Z} / 6 \oplus \mathbb{Z} / 2 .
\end{gathered}
$$

O segundo:

$$
\nu^{\prime} \circ \Sigma^{3}\left(\mu_{3}\right) \circ \Sigma^{12}\left(\nu^{\prime} \circ \eta_{6}\right) \stackrel{(4)}{=} \nu^{\prime} \circ \mu_{6} \circ \Sigma^{10}\left(2 \Sigma \nu_{4}\right) \circ \eta_{18}=\nu^{\prime} \circ 2 \mu_{6} \circ \Sigma^{11} \nu_{4} \circ \eta_{18} \stackrel{(10)}{=} 0 .
$$

Portanto,

$$
\begin{gathered}
\operatorname{Im} e v_{8}\left(\left[f_{1,0}\right]\right)=\operatorname{Im} e v_{8}\left(\left[f_{1,1}\right]\right)=\mathbb{Z} / 2, \\
\operatorname{Im} \partial_{8}=0 \\
\operatorname{Im} \phi=\mathbb{Z} / 6 \oplus \mathbb{Z} / 2, \\
T=\mathbb{Z} / 72 \oplus \mathbb{Z} / 2 \text { ou } T=\mathbb{Z} / 24 \oplus \mathbb{Z} / 6 \text { ou } T=\mathbb{Z} / 12 \oplus \mathbb{Z} / 6 \oplus \mathbb{Z} / 2, \\
\pi_{6}\left(\mathcal{G}_{f}\right) \approx \mathbb{Z} / 72 \oplus \mathbb{Z} / 2 \text { ou } \mathbb{Z} / 24 \oplus \mathbb{Z} / 6 \text { ou } \mathbb{Z} / 12 \oplus \mathbb{Z} / 6 \oplus \mathbb{Z} / 2 .
\end{gathered}
$$

Caso $\pi_{7}\left(\mathcal{G}_{f}\right)$. Considerando a seqüência no Lema 4.1.2 $(\operatorname{com} k=8)$,

$$
\begin{gathered}
\pi_{8}\left(S^{3}\right) \underset{\partial_{9}}{\longrightarrow} \pi_{20}\left(S^{3}\right) \longrightarrow \pi_{7}\left(\mathcal{G}_{f}\right) \underset{\text { ev8 }}{\longrightarrow} \pi_{7}\left(S^{3}\right) \underset{\partial_{8}}{\longrightarrow} \pi_{19}\left(S^{3}\right), \\
\mathbb{Z} / 2 \underset{\partial_{9}}{\longrightarrow} \mathbb{Z} / 12 \oplus \mathbb{Z} / 2 \oplus \mathbb{Z} / 2 \phi \longrightarrow \pi_{7}\left(\mathcal{G}_{f}\right) \underset{\text { ev }}{\longrightarrow} \mathbb{Z} / 2 \underset{\partial_{8}}{\longrightarrow} \mathbb{Z} / 6 \oplus \mathbb{Z} / 2 .
\end{gathered}
$$

É claro que

$$
\operatorname{Nuc} \phi=\operatorname{Im} \partial_{9}=0 \text { ou } \mathbb{Z} / 2 \text {. }
$$

Assim

$$
\operatorname{Im} \phi=(\mathbb{Z} / 12 \oplus \mathbb{Z} / 2 \oplus \mathbb{Z} / 2) / \operatorname{Nuc} \phi=\mathbb{Z} / 12 \oplus \mathbb{Z} / 2 \oplus \mathbb{Z} / 2 \text { ou } \mathbb{Z} / 12 \oplus \mathbb{Z} / 2
$$

Agora, como um grupo abeliano fintiamente gerado, $A=\pi_{7}\left(\mathcal{G}_{f}\right) \approx \mathbb{Z}^{m} \oplus T$, sendo $T$ o subgrupo de torção. Seja $f \neq c_{0}$ (recordemos que $\partial\left[f_{1,0}\right]=\mu_{3}, \partial\left[f_{0,1}\right]=\eta_{3} \circ \epsilon_{4}, \partial\left[f_{1,1}\right]=$ $\left.\mu_{3} \oplus \eta_{3} \circ \epsilon_{4}\right)$. Dos cálculos feitos no caso anterior, temos

$$
\operatorname{Im} e v_{8}\left(\left[f_{1,0}\right]\right)=\operatorname{Im} e v_{8}\left(\left[f_{0,1}\right]\right)=\operatorname{Im} e v_{8}\left(\left[f_{1,1}\right]\right)=\mathbb{Z} / 2
$$


Então

$$
\mathbb{Z} / 2=\operatorname{Im} e v_{8}=A / \operatorname{Nuc} e v_{8}=\left(\mathbb{Z}^{m} \oplus T\right) / \operatorname{Nuc} e v_{8}=\left(\mathbb{Z}^{m} \oplus T\right) / \operatorname{Im} \phi,
$$

implica que $m=0$ e $T=\mathbb{Z} / 24 \oplus \mathbb{Z} / 2 \oplus \mathbb{Z} / 2$ ou $T=\mathbb{Z} / 12 \oplus \mathbb{Z} / 4 \oplus \mathbb{Z} / 2$ ou $T=$ $\mathbb{Z} / 12 \oplus \mathbb{Z} / 2 \oplus \mathbb{Z} / 2 \oplus \mathbb{Z} / 2$ ou $T=\mathbb{Z} / 24 \oplus \mathbb{Z} / 2$ ou $T=\mathbb{Z} / 12 \oplus \mathbb{Z} / 4$ ou $T=\mathbb{Z} / 12 \oplus \mathbb{Z} / 2 \oplus \mathbb{Z} / 2$.

Para decidirmos qual é o caso, consideremos

$$
\operatorname{Im} \phi=(\mathbb{Z} / 12 \oplus \mathbb{Z} / 2 \oplus \mathbb{Z} / 2) / \operatorname{Nuc} \phi,
$$

Nuc $\phi=\operatorname{Im} \partial_{9}=0$ ou $\mathbb{Z} / 2$

e

$$
\operatorname{Im} \partial_{9}=(\mathbb{Z} / 2) / \operatorname{Im} e v_{9}
$$

Precisamos calcular Im $e v_{9}$. Pela Proposição 4.1 .8 (com $\left.k=9\right)$, precisamos resolver a equação

$$
\nu^{\prime} \circ \Sigma^{3} \partial \beta_{f}(2) \circ \Sigma^{12} \partial \omega(2)=0
$$

em $\pi_{20}\left(S^{3} ; 2\right)=\mathbb{Z} / 4\left[\bar{\epsilon}_{3}\right] \oplus \mathbb{Z} / 2\left[\bar{\mu}_{3}\right] \oplus \mathbb{Z} / 2\left[\eta_{3} \circ \mu_{4} \circ \sigma_{13}\right]$, com

$$
\partial \beta_{f}(2) \in \pi_{12}\left(S^{3} ; 2\right)=\mathbb{Z} / 2\left[\eta_{3} \circ \epsilon_{4}\right] \oplus \mathbb{Z} / 2\left[\mu_{3}\right]
$$

e

$$
\partial \omega(2) \in \pi_{8}\left(S^{3} ; 2\right)=\mathbb{Z} / 2\left[\nu^{\prime} \circ \eta_{6}^{2}\right] .
$$

Calculando então $\nu^{\prime} \circ \Sigma^{3} \partial \beta_{f}(2)$ para as duas possibilidades

$$
\nu^{\prime} \circ \Sigma^{3}\left(\eta_{3} \circ \epsilon_{4}\right)=\nu^{\prime} \circ \eta_{6} \circ \epsilon_{7}
$$

e

$$
\nu^{\prime} \circ \Sigma^{3}\left(\mu_{3}\right)=\nu^{\prime} \circ \mu_{6},
$$

ambos em $\pi_{15}\left(S^{3} ; 2\right)=\mathbb{Z} / 2\left[\nu^{\prime} \circ \eta_{6} \circ \epsilon_{7}\right] \oplus \mathbb{Z} / 2\left[\nu^{\prime} \circ \mu_{6}\right]$.

Compondo com o possível

$$
\Sigma^{12} \partial \omega=(n \bmod 2) \Sigma^{12}\left(\nu^{\prime}\right) \circ \eta_{18}^{2},
$$

obtemos elementos de $\pi_{19}\left(S^{3} ; 2\right)$.

O primeiro é:

$$
\begin{gathered}
\nu^{\prime} \circ \Sigma^{3}\left(\eta_{3} \circ \epsilon_{4}\right) \circ \Sigma^{12}\left(\nu^{\prime} \circ \eta_{6}^{2}\right) \stackrel{(4)}{=} \nu^{\prime} \circ \eta_{6} \circ \epsilon_{7} \circ \Sigma^{10}\left(2 \Sigma \nu_{4}\right) \circ \eta_{18}^{2} \\
=2 \nu^{\prime} \circ \eta_{6} \circ \epsilon_{7} \circ \Sigma^{11} \nu_{4} \circ \eta_{18}^{2} \stackrel{(1)}{=} 2 \nu^{\prime} \circ \eta_{6} \circ \nu_{7} \circ \epsilon_{10} \circ \eta_{18}^{2} \stackrel{(9)}{=} 0 .
\end{gathered}
$$

Logo,

$$
\begin{gathered}
\operatorname{Im} e v_{9}\left(\left[f_{0,1}\right]\right)=\mathbb{Z} / 2, \\
\operatorname{Im} \partial_{9}=0, \\
\operatorname{Im} \phi=\mathbb{Z} / 12 \oplus \mathbb{Z} / 2 \oplus \mathbb{Z} / 2,
\end{gathered}
$$


$T=\mathbb{Z} / 24 \oplus \mathbb{Z} / 2 \oplus \mathbb{Z} / 2$ ou $T=\mathbb{Z} / 12 \oplus \mathbb{Z} / 4 \oplus \mathbb{Z} / 2$ ou $T=\mathbb{Z} / 12 \oplus \mathbb{Z} / 2 \oplus \mathbb{Z} / 2 \oplus \mathbb{Z} / 2$, $\pi_{7}\left(\mathcal{G}_{f}\right)=\mathbb{Z} / 24 \oplus \mathbb{Z} / 2 \oplus \mathbb{Z} / 2$ ou $\mathbb{Z} / 12 \oplus \mathbb{Z} / 4 \oplus \mathbb{Z} / 2$ ou $\mathbb{Z} / 12 \oplus \mathbb{Z} / 2 \oplus \mathbb{Z} / 2 \oplus \mathbb{Z} / 2$.

O segundo:

$\nu^{\prime} \circ \Sigma^{3}\left(\mu_{3}\right) \circ \Sigma^{12}\left(\nu^{\prime} \circ \eta_{6}^{2}\right) \stackrel{(4)}{=} \nu^{\prime} \circ \mu_{6} \circ \Sigma^{10}\left(2 \Sigma \nu_{4}\right) \circ \eta_{18}^{2}=\nu^{\prime} \circ 2 \mu_{6} \circ \Sigma^{11} \nu_{4} \circ \eta_{18}^{2} \stackrel{(10)}{=} 0$.

Portanto,

$$
\begin{gathered}
\operatorname{Im} e v_{9}\left(\left[f_{1,0}\right]\right)=\operatorname{Im} e v_{9}\left(\left[f_{1,1}\right]\right)=\mathbb{Z} / 2, \\
\operatorname{Im} \partial_{9}=0,
\end{gathered}
$$

$\operatorname{Im} \phi=\mathbb{Z} / 12 \oplus \mathbb{Z} / 2 \oplus \mathbb{Z} / 2$,

$T=\mathbb{Z} / 24 \oplus \mathbb{Z} / 2 \oplus \mathbb{Z} / 2$ ou $T=\mathbb{Z} / 12 \oplus \mathbb{Z} / 4 \oplus \mathbb{Z} / 2$ ou $T=\mathbb{Z} / 12 \oplus \mathbb{Z} / 2 \oplus \mathbb{Z} / 2 \oplus \mathbb{Z} / 2$, $\pi_{7}\left(\mathcal{G}_{f}\right) \approx \mathbb{Z} / 24 \oplus \mathbb{Z} / 2 \oplus \mathbb{Z} / 2$ ou $\mathbb{Z} / 12 \oplus \mathbb{Z} / 4 \oplus \mathbb{Z} / 2$ ou $\mathbb{Z} / 12 \oplus \mathbb{Z} / 2 \oplus \mathbb{Z} / 2 \oplus \mathbb{Z} / 2$.

Caso $\pi_{8}\left(\mathcal{G}_{f}\right)$. Considerando a seqüência no Lema 4.1.2 (com $\left.k=9\right)$,

$$
\begin{gathered}
\pi_{9}\left(S^{3}\right) \underset{\partial_{10}}{\longrightarrow} \pi_{21}\left(S^{3}\right) \longrightarrow \pi_{8}\left(\mathcal{G}_{f}\right) \underset{e v_{9}}{\longrightarrow} \pi_{8}\left(S^{3}\right) \underset{\partial_{9}}{\longrightarrow} \pi_{20}\left(S^{3}\right), \\
\mathbb{Z} / 3 \underset{\partial_{10}}{\longrightarrow} \mathbb{Z} / 12 \oplus \mathbb{Z} / 2 \oplus \mathbb{Z} / 2 \underset{\phi}{\longrightarrow} \pi_{8}\left(\mathcal{G}_{f}\right) \underset{\text { ev }}{\longrightarrow} \mathbb{Z} / 2 \underset{\partial_{9}}{\longrightarrow} \mathbb{Z} / 12 \oplus \mathbb{Z} / 2 \oplus \mathbb{Z} / 2 .
\end{gathered}
$$

É claro que

$$
\operatorname{Nuc} \phi=\operatorname{Im} \partial_{10}=0 \text { ou } \mathbb{Z} / 3 \text {. }
$$

Assim

$\operatorname{Im} \phi=(\mathbb{Z} / 12 \oplus \mathbb{Z} / 2 \oplus \mathbb{Z} / 2) / \operatorname{Nuc} \phi=\mathbb{Z} / 12 \oplus \mathbb{Z} / 2 \oplus \mathbb{Z} / 2$ ou $\mathbb{Z} / 4 \oplus \mathbb{Z} / 2 \oplus \mathbb{Z} / 2$.

Agora, como um grupo abeliano finitamente gerado, $A=\pi_{8}\left(\mathcal{G}_{f}\right) \approx \mathbb{Z}^{m} \oplus T$, sendo $T$ o subgrupo de torção. Seja $f \neq c_{0}$ (recordemos que $\partial\left[f_{1,0}\right]=\mu_{3}, \partial\left[f_{0,1}\right]=\eta_{3} \circ \epsilon_{4}, \partial\left[f_{1,1}\right]=$ $\left.\mu_{3} \oplus \eta_{3} \circ \epsilon_{4}\right)$. Dos cálculos feitos no caso anterior, temos

$$
\operatorname{Im} e v_{9}\left(\left[f_{1,0}\right]\right)=\operatorname{Im} e v_{9}\left(\left[f_{0,1}\right]\right)=\operatorname{Im} e v_{9}\left(\left[f_{1,1}\right]\right)=\mathbb{Z} / 2
$$

Então

$$
\mathbb{Z} / 2=\operatorname{Im} e v_{9}=A / \operatorname{Nuc} e v_{9}=\left(\mathbb{Z}^{m} \oplus T\right) / \operatorname{Nuc} e v_{9}=\left(\mathbb{Z}^{m} \oplus T\right) / \operatorname{Im} \phi,
$$

implica que $m=0$ e $T=\mathbb{Z} / 24 \oplus \mathbb{Z} / 2 \oplus \mathbb{Z} / 2$ ou $T=\mathbb{Z} / 12 \oplus \mathbb{Z} / 4 \oplus \mathbb{Z} / 2$ ou $T=$ $\mathbb{Z} / 12 \oplus \mathbb{Z} / 2 \oplus \mathbb{Z} / 2 \oplus \mathbb{Z} / 2$ ou $T=\mathbb{Z} / 8 \oplus \mathbb{Z} / 2 \oplus \mathbb{Z} / 2$ ou $T=\mathbb{Z} / 4 \oplus \mathbb{Z} / 4 \oplus \mathbb{Z} / 2$ ou $T=\mathbb{Z} / 4 \oplus \mathbb{Z} / 2 \oplus \mathbb{Z} / 2 \oplus \mathbb{Z} / 2$.

Para decidirmos qual é o caso, consideremos que

$$
\operatorname{Im} \phi=(\mathbb{Z} / 12 \oplus \mathbb{Z} / 2 \oplus \mathbb{Z} / 2) / \operatorname{Nuc} \phi,
$$


$\operatorname{Nuc} \phi=\operatorname{Im} \partial_{10}=0$ ou $\mathbb{Z} / 3$

e

$$
\operatorname{Im} \partial_{10}=(\mathbb{Z} / 3) / \operatorname{Im} e v_{10}
$$

Precisamos calcular Im $e v_{10}$. Pela Proposição 4.1 .8 (com $\left.k=10\right)$, precisamos resolver a equação

$$
\nu^{\prime} \circ \Sigma^{3} \partial \beta_{f}(2) \circ \Sigma^{12} \partial \omega(2)=0
$$

em $\pi_{21}\left(S^{3} ; 2\right)=\mathbb{Z} / 4\left[\mu^{\prime} \circ \sigma_{14}\right] \oplus \mathbb{Z} / 2\left[\nu^{\prime} \circ \bar{\epsilon}_{6}\right] \oplus \mathbb{Z} / 2\left[\eta_{3} \circ \bar{\mu}_{4}\right]$, com

$$
\partial \beta_{f}(2) \in \pi_{12}\left(S^{3} ; 2\right)=\mathbb{Z} / 2\left[\eta_{3} \circ \epsilon_{4}\right] \oplus \mathbb{Z} / 2\left[\mu_{3}\right]
$$

e

$$
\partial \omega(2) \in \pi_{9}\left(S^{3} ; 2\right)=0 .
$$

Disso, temos que para qualquer $f$

$$
\begin{gathered}
\operatorname{Im} e v_{10}=\mathbb{Z} / 3, \\
\operatorname{Im} \partial_{10}=0, \\
\operatorname{Im} \phi=\mathbb{Z} / 12 \oplus \mathbb{Z} / 2 \oplus \mathbb{Z} / 2,
\end{gathered}
$$

$T=\mathbb{Z} / 24 \oplus \mathbb{Z} / 2 \oplus \mathbb{Z} / 2$ ou $T=\mathbb{Z} / 12 \oplus \mathbb{Z} / 4 \oplus \mathbb{Z} / 2$ ou $T=\mathbb{Z} / 12 \oplus \mathbb{Z} / 2 \oplus \mathbb{Z} / 2 \oplus \mathbb{Z} / 2$,

$$
\pi_{8}\left(\mathcal{G}_{f}\right) \approx \mathbb{Z} / 24 \oplus \mathbb{Z} / 2 \oplus \mathbb{Z} / 2 \text { ou } \mathbb{Z} / 12 \oplus \mathbb{Z} / 4 \oplus \mathbb{Z} / 2 \text { ou } \mathbb{Z} / 12 \oplus \mathbb{Z} / 2 \oplus \mathbb{Z} / 2 \oplus \mathbb{Z} / 2 .
$$

Caso $\pi_{9}\left(\mathcal{G}_{f}\right)$. Considerando a seqüência no Lema 4.1.2 (com $\left.k=10\right)$,

$$
\begin{gathered}
\pi_{10}\left(S^{3}\right) \underset{\partial_{11}}{\longrightarrow} \pi_{22}\left(S^{3}\right) \longrightarrow \pi_{9}\left(\mathcal{G}_{f}\right) \underset{e v_{10}}{\longrightarrow} \pi_{9}\left(S^{3}\right) \underset{\partial_{10}}{\longrightarrow} \pi_{21}\left(S^{3}\right), \\
\mathbb{Z} / 15 \underset{\partial_{11}}{\longrightarrow} \mathbb{Z} / 132 \oplus \mathbb{Z} / 2 \underset{\phi}{\longrightarrow} \pi_{9}\left(\mathcal{G}_{f}\right) \underset{\text { ev } 10}{\longrightarrow} \mathbb{Z} / 3 \underset{\partial_{10}}{\longrightarrow} \mathbb{Z} / 12 \oplus \mathbb{Z} / 2 \oplus \mathbb{Z} / 2 .
\end{gathered}
$$

É claro que

$$
\text { Nuc } \phi=\operatorname{Im} \partial_{11}=0 \text { ou } \mathbb{Z} / 3 \text {. }
$$

Assim

$$
\operatorname{Im} \phi=(\mathbb{Z} / 132 \oplus \mathbb{Z} / 2) / \text { Nuc } \phi=\mathbb{Z} / 132 \oplus \mathbb{Z} / 2 \text { ou } \mathbb{Z} / 44 \oplus \mathbb{Z} / 2 \text {. }
$$

Agora, como um grupo abeliano finitamente gerado, $A=\pi_{9}\left(\mathcal{G}_{f}\right) \approx \mathbb{Z}^{m} \oplus T$, sendo $T$ o subgrupo de torção. Seja $f \neq c_{0}$ (recordemos que $\partial\left[f_{1,0}\right]=\mu_{3}, \partial\left[f_{0,1}\right]=\eta_{3} \circ \epsilon_{4}, \partial\left[f_{1,1}\right]=$ $\left.\mu_{3} \oplus \eta_{3} \circ \epsilon_{4}\right)$. Dos cálculos feitos no caso anterior, temos

$$
\operatorname{Im} e v_{10}\left(\left[f_{1,0}\right]\right)=\operatorname{Im} e v_{10}\left(\left[f_{0,1}\right]\right)=\operatorname{Im} e v_{10}\left(\left[f_{1,1}\right]\right)=\mathbb{Z} / 3
$$

Então

$$
\mathbb{Z} / 3=\operatorname{Im} e v_{10}=A / \operatorname{Nuc} e v_{10}=\left(\mathbb{Z}^{m} \oplus T\right) / \operatorname{Nuc} e v_{10}=\left(\mathbb{Z}^{m} \oplus T\right) / \operatorname{Im} \phi,
$$


implica que $m=0$ e $T=\mathbb{Z} / 396 \oplus \mathbb{Z} / 2$ ou $T=\mathbb{Z} / 132 \oplus \mathbb{Z} / 6$ ou $T=\mathbb{Z} / 132 \oplus \mathbb{Z} / 2$ ou $T=\mathbb{Z} / 44 \oplus \mathbb{Z} / 6$.

Para decidir qual é o caso, consideremos o seguinte

$$
\begin{gathered}
\operatorname{Im} \phi=(\mathbb{Z} / 132 \oplus \mathbb{Z} / 2) / \text { Nuc } \phi, \\
\text { Nuc } \phi=\operatorname{Im} \partial_{11}=0 \text { ou } \mathbb{Z} / 3
\end{gathered}
$$

e

$$
\operatorname{Im} \partial_{10}=(\mathbb{Z} / 15) / \operatorname{Im} e v_{11}
$$

Precisamos calcular Im $e v_{11}$. Pela Proposição 4.1 .8 (com $\left.k=11\right)$, precisamos resolver a equação

$$
\nu^{\prime} \circ \Sigma^{3} \partial \beta_{f}(2) \circ \Sigma^{12} \partial \omega(2)=0
$$

em $\pi_{22}\left(S^{3} ; 2\right)=\mathbb{Z} / 4\left[\bar{\mu}^{\prime}\right] \oplus \mathbb{Z} / 2\left[\nu^{\prime} \circ \eta_{6} \circ \sigma_{15}\right]$, com

$$
\partial \beta_{f}(2) \in \pi_{12}\left(S^{3} ; 2\right)=\mathbb{Z} / 2\left[\eta_{3} \circ \epsilon_{4}\right] \oplus \mathbb{Z} / 2\left[\mu_{3}\right]
$$

e

$$
\partial \omega(2) \in \pi_{10}\left(S^{3} ; 2\right)=0 .
$$

Disso, temos que para qualquer $f$

$$
\begin{gathered}
\operatorname{Im} e v_{11}=\mathbb{Z} / 15, \\
\operatorname{Im} \partial_{11}=0, \\
\operatorname{Im} \phi=\mathbb{Z} / 132 \oplus \mathbb{Z} / 2, \\
T=\mathbb{Z} / 392 \oplus \mathbb{Z} / 2 \text { ou } T=\mathbb{Z} / 132 \oplus \mathbb{Z} / 6, \\
\pi_{9}\left(\mathcal{G}_{f}\right) \approx \mathbb{Z} / 392 \oplus \mathbb{Z} / 2 \text { ou } \mathbb{Z} / 132 \oplus \mathbb{Z} / 6 .
\end{gathered}
$$

Observação 4.3.10. Podemos ver pelas provas dos Lemas acima que

$$
\nu^{\prime} \circ \Sigma^{3}\left(\eta_{3} \circ \epsilon_{4}\right) \circ \Sigma^{12} \partial \omega(2)=0
$$

para qualquer um dos $\partial \omega(2)$ usados (notação da Proposição 4.1.8). Continuando os cálculos, agora só para essa composição, esperamos encontrar pelo menos um caso no qual ela seja diferente de zero. Caso isso ocorra, poderemos ter então $\pi_{n}\left(G_{f_{(1,0)}}\right) \not \approx \pi_{n}\left(G_{f_{(1,1)}}\right)$, para algum $n$.

Usando a Proposição 4.1.8 em cada uma dessas composições, consideremos cada caso (recordando que o número indicado em cada igualdade se refere a relação encontrada no Apêndice deste capítulo): 
Caso $k=12$. Temos

$$
\nu^{\prime} \circ \Sigma^{3} \partial \beta_{f}(2) \circ \Sigma^{12} \partial \omega(2)=0
$$

em $\pi_{23}\left(S^{3} ; 2\right)$. Aqui, $\partial \omega(2)$ está em $\pi_{11}\left(S^{3} ; 2\right)=\mathbb{Z} / 2\left[\epsilon_{3}\right]$.

Compondo

$$
\begin{gathered}
\nu^{\prime} \circ \Sigma^{3}\left(\eta_{3} \circ \epsilon_{4}\right) \circ \Sigma^{12}\left(\epsilon_{3}\right)=\nu^{\prime} \circ \eta_{6} \circ \epsilon_{7} \circ \epsilon_{15} \stackrel{(11)}{=} 6 \nu^{\prime} \circ \epsilon_{6} \circ \eta_{14} \circ \epsilon_{15} \\
\stackrel{(16)}{=} \epsilon^{\prime} \circ \eta_{13} \circ \eta_{14} \circ \epsilon_{15} \stackrel{(14)}{=} \epsilon^{\prime} \circ 4\left(\nu_{3} \circ \sigma_{16}\right)=4 \epsilon^{\prime} \circ \nu_{3} \circ \sigma_{16}=0 .
\end{gathered}
$$

Caso $k=13$. Temos

$$
\nu^{\prime} \circ \Sigma^{3} \partial \beta_{f}(2) \circ \Sigma^{12} \partial \omega(2)=0
$$

em $\pi_{24}\left(S^{3} ; 2\right)$. Aqui, $\partial \omega(2)$ está em $\pi_{12}\left(S^{3} ; 2\right)=\mathbb{Z} / 2\left[\mu_{3}\right] \oplus \mathbb{Z} / 2\left[\eta_{3} \circ \epsilon_{4}\right]$.

Compondo com $\partial \omega(2)=\mu_{3}$

$$
\begin{gathered}
\nu^{\prime} \circ \Sigma^{3}\left(\eta_{3} \circ \epsilon_{4}\right) \circ \Sigma^{12}\left(\mu_{3}\right)=\nu^{\prime} \circ \eta_{6} \circ \epsilon_{7} \circ \mu_{15} \stackrel{(11)}{=} \nu^{\prime} \circ \epsilon_{6} \circ \eta_{14} \circ \mu_{15} \\
\stackrel{(16)}{=} \epsilon^{\prime} \circ \eta_{13} \circ \eta_{14} \circ \mu_{15} \stackrel{(17)}{=} \epsilon^{\prime} \circ 4\left(\zeta_{13}\right)=4 \epsilon^{\prime} \circ \zeta_{13}=0 .
\end{gathered}
$$

Compondo com $\partial \omega(2)=\eta_{3} \circ \epsilon_{4}$

$$
\begin{gathered}
\nu^{\prime} \circ \Sigma^{3}\left(\eta_{3} \circ \epsilon_{4}\right) \circ \Sigma^{12}\left(\eta_{3} \circ \epsilon_{4}\right)=\nu^{\prime} \circ \eta_{6} \circ \epsilon_{7} \circ \eta_{15} \circ \epsilon_{16} \stackrel{(11)}{=} \nu^{\prime} \circ \epsilon_{6} \circ \eta_{7} \circ \epsilon_{8} \circ \epsilon_{16} \\
\stackrel{(14)}{=} \nu^{\prime} \circ 4\left(\nu_{6} \circ \sigma_{9}\right) \circ \epsilon_{16}=4 \nu^{\prime} \circ \nu_{6} \circ \sigma_{9} \circ \epsilon_{16}=0 .
\end{gathered}
$$

Caso $k=14$. Temos

$$
\nu^{\prime} \circ \Sigma^{3} \partial \beta_{f}(2) \circ \Sigma^{12} \partial \omega(2)=0
$$

em $\pi_{25}\left(S^{3} ; 2\right)$. Aqui, $\partial \omega(2)$ entá em $\pi_{13}\left(S^{3} ; 2\right)=\mathbb{Z} / 4\left[\epsilon^{\prime}\right] \oplus \mathbb{Z} / 2\left[\eta_{3} \circ \mu_{4}\right]$.

Compondo com $\partial \omega(2)=\epsilon^{\prime}$

$$
\begin{aligned}
\nu^{\prime} \circ \Sigma^{3}\left(\eta_{3} \circ \epsilon_{4}\right) \circ \Sigma^{12}\left(\epsilon^{\prime}\right) & =\nu^{\prime} \circ \eta_{6} \circ \epsilon_{7} \circ \Sigma^{12}\left(\epsilon^{\prime}\right) \stackrel{(14)}{=} \nu^{\prime} \circ \eta_{6} \circ \epsilon_{7} \circ \Sigma^{10}\left(2 \nu_{5} \circ \sigma_{8}\right) \\
& =\nu^{\prime} \circ 2 \eta_{6} \circ \epsilon_{7} \circ \nu_{15} \circ \sigma_{18}=0 .
\end{aligned}
$$

Compondo com $\partial \omega(2)=\eta_{3} \circ \mu_{4}$

$$
\begin{gathered}
\nu^{\prime} \circ \Sigma^{3}\left(\eta_{3} \circ \epsilon_{4}\right) \circ \Sigma^{12}\left(\eta_{3} \circ \mu_{4}\right)=\nu^{\prime} \circ \eta_{6} \circ \epsilon_{7} \circ \eta_{15} \circ \mu_{16} \stackrel{(11)}{=} \nu^{\prime} \circ \epsilon_{6} \circ \eta_{7} \circ \epsilon_{8} \circ \mu_{16} \\
\stackrel{(14)}{=} \nu^{\prime} \circ 4\left(\nu_{6} \circ \sigma_{9}\right) \circ \mu_{16}=4 \nu^{\prime} \circ \nu_{6} \circ \sigma_{9} \circ \mu_{16}=0 .
\end{gathered}
$$

\footnotetext{
${ }^{6}$ Recorde que os números que seguem nas igualdades se referem as relações apresentadas no Apêndice deste capítulo.
} 
Caso $k=15$. Temos

$$
\nu^{\prime} \circ \Sigma^{3} \partial \beta_{f}(2) \circ \Sigma^{12} \partial \omega(2)=0
$$

em $\pi_{26}\left(S^{3} ; 2\right)$. Aqui, $\partial \omega(2)$ está em $\pi_{14}\left(S^{3} ; 2\right)=\mathbb{Z} / 4\left[\mu^{\prime}\right] \oplus \mathbb{Z} / 2\left[\epsilon_{3} \circ \nu_{11}\right] \oplus \mathbb{Z} / 2\left[\nu^{\prime} \circ \epsilon_{6}\right]$.

Compondo com $\partial \omega(2)=\mu^{\prime}$

$$
\begin{aligned}
\nu^{\prime} \circ \Sigma^{3}\left(\eta_{3} \circ \epsilon_{4}\right) \circ \Sigma^{12}\left(\mu^{\prime}\right) & =\nu^{\prime} \circ \eta_{6} \circ \epsilon_{7} \circ \Sigma^{12}\left(\mu^{\prime}\right) \stackrel{(17)}{=} \nu^{\prime} \circ \eta_{6} \circ \epsilon_{7} \circ \Sigma^{10}\left(2 \zeta_{5}\right) \\
& =\nu^{\prime} \circ 2 \eta_{6} \circ \epsilon_{7} \circ \zeta_{15}=0 .
\end{aligned}
$$

Compondo com $\partial \omega(2)=\epsilon_{3} \circ \nu_{11}$

$$
\begin{aligned}
& \nu^{\prime} \circ \Sigma^{3}\left(\eta_{3} \circ \epsilon_{4}\right) \circ \Sigma^{12}\left(\epsilon_{3} \circ \nu_{11}\right)=\nu^{\prime} \circ \eta_{6} \circ \epsilon_{7} \circ \epsilon_{15} \circ \nu_{23} \stackrel{(11)}{=} \nu^{\prime} \circ \epsilon_{6} \circ \eta_{14} \circ \epsilon_{15} \circ \nu_{23} \\
& \stackrel{(16)}{=} \epsilon^{\prime} \circ \eta_{13} \circ \eta_{14} \circ \epsilon_{15} \circ \nu_{23} \stackrel{(14)}{=} \epsilon^{\prime} \circ 4\left(\nu_{13} \circ \sigma_{16}\right) \circ \nu_{23}=4 \epsilon^{\prime} \circ \nu_{13} \circ \sigma_{16} \circ \nu_{23}=0 .
\end{aligned}
$$

Compondo com $\partial \omega(2)=\nu^{\prime} \circ \epsilon_{6}$

$$
\begin{gathered}
\nu^{\prime} \circ \Sigma^{3}\left(\eta_{3} \circ \epsilon_{4}\right) \circ \Sigma^{12}\left(\nu^{\prime} \circ \epsilon_{6}\right) \stackrel{(16)}{=} \nu^{\prime} \circ \eta_{6} \circ \epsilon_{7} \circ \Sigma^{12}\left(\epsilon^{\prime} \circ \eta_{13}\right)=\nu^{\prime} \circ \eta_{6} \circ \epsilon_{7} \circ \Sigma^{12} \epsilon^{\prime} \circ \eta_{25} \\
\stackrel{(1)}{=} \nu^{\prime} \circ \eta_{6} \circ \epsilon_{7} \circ \eta_{15} \circ \Sigma^{13} \epsilon^{\prime} \stackrel{(1)}{=} \nu^{\prime} \circ \eta_{6} \circ \eta_{7} \circ \epsilon_{8} \circ \Sigma^{13} \epsilon^{\prime} \stackrel{(14)}{=} \nu^{\prime} \circ 4\left(\nu_{6} \circ \sigma_{9}\right) \circ \Sigma^{13} \epsilon^{\prime} \\
=4 \nu^{\prime} \circ \nu_{6} \circ \sigma_{9} \circ \Sigma^{13} \epsilon^{\prime}=0 .
\end{gathered}
$$

Caso $k=16$. Temos

$$
\nu^{\prime} \circ \Sigma^{3} \partial \beta_{f}(2) \circ \Sigma^{12} \partial \omega(2)=0
$$

em $\pi_{27}\left(S^{3} ; 2\right)$. Aqui, $\partial \omega(2)$ está em $\pi_{15}\left(S^{3} ; 2\right)=\mathbb{Z} / 2\left[\nu^{\prime} \circ \mu_{6}\right] \oplus \mathbb{Z} / 2\left[\nu^{\prime} \circ \eta_{6} \circ \epsilon_{7}\right]$.

Compondo com $\partial \omega(2)=\nu^{\prime} \circ \mu_{6}$

$$
\begin{gathered}
\nu^{\prime} \circ \Sigma^{3}\left(\eta_{3} \circ \epsilon_{4}\right) \circ \Sigma^{12}\left(\nu^{\prime} \circ \mu_{6}\right)=\nu^{\prime} \circ \eta_{6} \circ \epsilon_{7} \circ \Sigma^{12} \nu^{\prime} \circ \mu_{18} \stackrel{(1)}{=} \nu^{\prime} \circ \eta_{6} \circ \Sigma^{4} \nu^{\prime} \circ \epsilon_{10} \circ \mu_{18} \\
\stackrel{(4)}{=} \nu^{\prime} \circ \eta_{6} \circ 2 \nu_{7} \circ \epsilon_{10} \circ \mu_{18}=2 \nu^{\prime} \circ \eta_{6} \circ \nu_{7} \circ \epsilon_{10} \circ \mu_{18} \stackrel{(9)}{=} 0 .
\end{gathered}
$$

Compondo com $\partial \omega(2)=\nu^{\prime} \circ \eta_{6} \circ \epsilon_{7}$

$$
\begin{gathered}
\nu^{\prime} \circ \Sigma^{3}\left(\eta_{3} \circ \epsilon_{4}\right) \circ \Sigma^{12}\left(\nu^{\prime} \circ \eta_{6} \circ \epsilon_{7}\right)=\nu^{\prime} \circ \eta_{6} \circ \epsilon_{7} \circ \Sigma^{12} \nu^{\prime} \circ \eta_{18} \circ \epsilon_{19} \stackrel{(1)}{=} \nu^{\prime} \circ \eta_{6} \circ \Sigma^{4} \nu^{\prime} \circ \epsilon_{10} \circ \eta_{18} \circ \epsilon_{19} \\
\stackrel{(4)}{=} \nu^{\prime} \circ \eta_{6} \circ 2 \nu_{7} \circ \epsilon_{10} \circ \eta_{18} \circ \epsilon_{19}=2 \nu^{\prime} \circ \eta_{6} \circ \nu_{7} \circ \epsilon_{10} \circ \eta_{18} \circ \epsilon_{19} \stackrel{(9)}{=} 0 .
\end{gathered}
$$

Caso $k=17$. Temos

$$
\nu^{\prime} \circ \Sigma^{3} \partial \beta_{f}(2) \circ \Sigma^{12} \partial \omega(2)=0
$$


em $\pi_{28}\left(S^{3} ; 2\right)$. Aqui, $\partial \omega(2)$ está em $\pi_{16}\left(S^{3} ; 2\right)=\mathbb{Z} / 2\left[\nu^{\prime} \circ \eta_{6} \circ \mu_{7}\right]$.

Compondo com $\partial \omega(2)=\nu^{\prime} \circ \eta_{6} \circ \mu_{7}$

$$
\begin{gathered}
\nu^{\prime} \circ \Sigma^{3}\left(\eta_{3} \circ \epsilon_{4}\right) \circ \Sigma^{12}\left(\nu^{\prime} \circ \eta_{6} \circ \mu_{7}\right)=\nu^{\prime} \circ \eta_{6} \circ \epsilon_{7} \circ \Sigma^{12} \nu^{\prime} \circ \eta_{18} \circ \mu_{19} \stackrel{(1)}{=} \nu^{\prime} \circ \eta_{6} \circ \Sigma^{4} \nu^{\prime} \circ \epsilon_{10} \circ \eta_{18} \circ \mu_{19} \\
\stackrel{(4)}{=} \nu^{\prime} \circ \eta_{6} \circ 2 \nu_{7} \circ \epsilon_{10} \circ \eta_{18} \circ \mu_{19}=2 \nu^{\prime} \circ \eta_{6} \circ \nu_{7} \circ \epsilon_{10} \circ \eta_{18} \circ \mu_{19} \stackrel{(9)}{=} 0 .
\end{gathered}
$$

Caso $k=18$. Temos

$$
\nu^{\prime} \circ \Sigma^{3} \partial \beta_{f}(2) \circ \Sigma^{12} \partial \omega(2)=0
$$

em $\pi_{29}\left(S^{3} ; 2\right)$. Aqui, $\partial \omega(2)$ está em $\pi_{17}\left(S^{3} ; 2\right)=\mathbb{Z} / 2\left[\epsilon_{3} \circ \nu_{11}^{2}\right]$.

Compondo com $\partial \omega(2)=\epsilon_{3} \circ \nu_{11}^{2}$

$$
\begin{gathered}
\nu^{\prime} \circ \Sigma^{3}\left(\eta_{3} \circ \epsilon_{4}\right) \circ \Sigma^{12}\left(\epsilon_{3} \circ \nu_{11}^{2}\right)=\nu^{\prime} \circ \eta_{6} \circ \epsilon_{7} \circ \epsilon_{15} \circ \nu_{23}^{2}=\nu^{\prime} \circ \eta_{6} \circ \epsilon_{7} \circ \epsilon_{15} \circ \nu_{23} \circ \nu_{26} \\
\stackrel{(1)}{=} \nu^{\prime} \circ \eta_{6} \circ \epsilon_{7} \circ \nu_{15} \circ \epsilon_{18} \circ \nu_{26} \stackrel{(1)}{=} \nu^{\prime} \circ \eta_{6} \circ \nu_{7} \circ \epsilon_{10} \circ \epsilon_{18} \circ \nu_{26} \stackrel{(9)}{=} 0 .
\end{gathered}
$$

Caso $k=19$. Temos

$$
\nu^{\prime} \circ \Sigma^{3} \partial \beta_{f}(2) \circ \Sigma^{12} \partial \omega(2)=0
$$

em $\pi_{30}\left(S^{3} ; 2\right)$. Aqui, $\partial \omega(2)$ está em $\pi_{18}\left(S^{3} ; 2\right)=\mathbb{Z} / 2\left[\bar{\epsilon}_{3}\right]$.

Compondo com $\partial \omega(2)=\bar{\epsilon}_{3}$

$$
\begin{gathered}
\nu^{\prime} \circ \Sigma^{3}\left(\eta_{3} \circ \epsilon_{4}\right) \circ \Sigma^{12}\left(\bar{\epsilon}_{3}\right)=\nu^{\prime} \circ \eta_{6} \circ \epsilon_{7} \circ \bar{\epsilon}_{15} \stackrel{(1)}{=} \nu^{\prime} \circ \epsilon_{6} \circ \eta_{14} \circ \bar{\epsilon}_{15} \\
\stackrel{(16)}{=} \epsilon^{\prime} \circ \eta_{13} \circ \eta_{14} \circ \bar{\epsilon}_{15} \stackrel{(16)}{=} \epsilon^{\prime} \circ \eta_{13} \circ \nu_{14} \circ \sigma_{17} \circ \nu_{24}^{2} \stackrel{(9)}{=} 0 .
\end{gathered}
$$

Caso $k=20$. Temos

$$
\nu^{\prime} \circ \Sigma^{3} \partial \beta_{f}(2) \circ \Sigma^{12} \partial \omega(2)=0
$$

em $\pi_{31}\left(S^{3} ; 2\right)$. Aqui, $\partial \omega(2)$ está em $\pi_{19}\left(S^{3} ; 2\right)=\mathbb{Z} / 2\left[\mu_{3} \circ \sigma_{12}\right] \oplus \mathbb{Z} / 2\left[\eta_{3} \circ \bar{\epsilon}_{4}\right]$.

Compondo com $\partial \omega(2)=\mu_{3} \circ \sigma_{12}$

$$
\begin{gathered}
\nu^{\prime} \circ \Sigma^{3}\left(\eta_{3} \circ \epsilon_{4}\right) \circ \Sigma^{12}\left(\mu_{3} \circ \sigma_{12}\right)=\nu^{\prime} \circ \eta_{6} \circ \epsilon_{7} \circ \mu_{15} \circ \sigma_{24} \stackrel{(1)}{=} \nu^{\prime} \circ \epsilon_{6} \circ \eta_{14} \circ \mu_{15} \circ \sigma_{24} \\
\stackrel{(17)}{=} \epsilon^{\prime} \circ 4 \zeta_{13} \circ \sigma_{24}=4 \epsilon^{\prime} \circ \zeta_{13} \circ \sigma_{24}=0 .
\end{gathered}
$$

Compondo com $\partial \omega(2)=\eta_{3} \circ \bar{\epsilon}_{4}$

$$
\begin{gathered}
\nu^{\prime} \circ \Sigma^{3}\left(\eta_{3} \circ \epsilon_{4}\right) \circ \Sigma^{12}\left(\eta_{3} \circ \bar{\epsilon}_{4}\right)=\nu^{\prime} \circ \eta_{6} \circ \epsilon_{7} \circ \eta_{15} \circ \bar{\epsilon}_{16} \stackrel{(1)}{=} \nu^{\prime} \circ \eta_{6} \circ \eta_{7} \circ \epsilon_{8} \circ \bar{\epsilon}_{16} \\
\stackrel{(14)}{=} \nu^{\prime} \circ 4\left(\nu_{6} \circ \sigma_{9}\right) \circ \bar{\epsilon}_{16}=4 \nu^{\prime} \circ \nu_{6} \circ \sigma_{9} \circ \bar{\epsilon}_{16}=0 .
\end{gathered}
$$


Caso $k=21$. Temos

$$
\nu^{\prime} \circ \Sigma^{3} \partial \beta_{f}(2) \circ \Sigma^{12} \partial \omega(2)=0
$$

em $\pi_{32}\left(S^{3} ; 2\right)$. Aqui, $\partial \omega(2)$ está em $\pi_{20}\left(S^{3} ; 2\right)=\mathbb{Z} / 4\left[\bar{\epsilon}^{\prime}\right] \oplus \mathbb{Z} / 2\left[\bar{\mu}_{3}\right] \oplus \mathbb{Z} / 2\left[\eta_{3} \circ \mu_{4} \circ \sigma_{13}\right]$.

Compondo com $\partial \omega(2)=\bar{\epsilon}^{\prime}$

$$
\begin{gathered}
\nu^{\prime} \circ \Sigma^{3}\left(\eta_{3} \circ \epsilon_{4}\right) \circ \Sigma^{12}\left(\bar{\epsilon}^{\prime}\right) \stackrel{(21)}{=} \nu^{\prime} \circ \eta_{6} \circ \epsilon_{7} \circ \Sigma^{12} \nu^{\prime} \circ \kappa_{18} \stackrel{(1)}{=} \nu^{\prime} \circ \eta_{6} \circ \Sigma^{4} \nu^{\prime} \circ \epsilon_{10} \circ \kappa_{18} \\
\stackrel{(4)}{=} \nu^{\prime} \circ \eta_{6} \circ 2 \Sigma^{3} \nu_{4} \circ \epsilon_{10} \circ \kappa_{18}=2 \nu^{\prime} \circ \eta_{6} \circ \nu_{7} \circ \epsilon_{10} \circ \kappa_{18} \stackrel{(9)}{=} 0 .
\end{gathered}
$$

Compondo com $\partial \omega(2)=\bar{\mu}_{3}$

$$
\begin{gathered}
\nu^{\prime} \circ \Sigma^{3}\left(\eta_{3} \circ \epsilon_{4}\right) \circ \Sigma^{12}\left(\bar{\mu}_{3}\right)=\nu^{\prime} \circ \eta_{6} \circ \epsilon_{7} \circ \bar{\mu}_{15} \stackrel{(1)}{=} \nu^{\prime} \circ \epsilon_{6} \circ \eta_{14} \circ \bar{\mu}_{15} \\
\stackrel{(16)}{=} \epsilon^{\prime} \circ \eta_{13} \circ \eta_{14} \circ \bar{\mu}_{15} \stackrel{(22)}{=} \epsilon^{\prime} \circ \Sigma^{10}\left(\eta_{3}^{2} \circ \bar{\mu}_{15}\right)=\epsilon^{\prime} \circ \Sigma^{10}\left(2 \bar{\mu}^{\prime}\right) \\
\stackrel{(22)}{=} 2 \epsilon^{\prime} \circ \Sigma^{8}\left(\Sigma^{2} \bar{\mu}^{\prime}\right)=2 \epsilon^{\prime} \circ \Sigma^{8}\left(2 \bar{\zeta}_{5}\right)=4 \epsilon^{\prime} \circ \bar{\zeta}_{13}=0 .
\end{gathered}
$$

Compondo com $\partial \omega(2)=\eta_{3} \circ \mu_{4} \circ \sigma_{13}$

$$
\nu^{\prime} \circ \Sigma^{3}\left(\eta_{3} \circ \epsilon_{4}\right) \circ \Sigma^{12}\left(\eta_{3} \circ \mu_{4} \circ \sigma_{13}\right)=\nu^{\prime} \circ \eta_{6} \circ \epsilon_{7} \circ \eta_{15} \circ \mu_{16} \circ \sigma_{25}
$$

$\stackrel{(1)}{=} \nu^{\prime} \circ \eta_{6} \circ \eta_{7} \circ \epsilon_{8} \circ \mu_{16} \circ \sigma_{25} \stackrel{(14)}{=} \nu^{\prime} \circ 4\left(\nu_{6} \circ \sigma_{9}\right) \circ \mu_{16} \circ \sigma_{25}=4 \nu^{\prime} \circ \nu_{6} \circ \sigma_{9} \circ \mu_{16} \circ \sigma_{25}=0$.

Caso $k=22$. Temos

$$
\nu^{\prime} \circ \Sigma^{3} \partial \beta_{f}(2) \circ \Sigma^{12} \partial \omega(2)=0
$$

em $\pi_{33}\left(S^{3} ; 2\right)$. Aqui, $\partial \omega(2)$ está em $\pi_{21}\left(S^{3} ; 2\right)=\mathbb{Z} / 4\left[\mu^{\prime} \circ \sigma_{14}\right] \oplus \mathbb{Z} / 2\left[\nu^{\prime} \circ \bar{\epsilon}_{6}\right] \oplus \mathbb{Z} / 2\left[\eta_{3} \circ \bar{\mu}_{4}\right]$.

Compondo com $\partial \omega(2)=\mu^{\prime} \circ \sigma_{14}$

$$
\begin{gathered}
\nu^{\prime} \circ \Sigma^{3}\left(\eta_{3} \circ \epsilon_{4}\right) \circ \Sigma^{12}\left(\mu^{\prime} \circ \sigma_{14}\right)=\nu^{\prime} \circ \eta_{6} \circ \epsilon_{7} \circ \Sigma^{12} \mu^{\prime} \circ \sigma_{26} \\
\stackrel{(1)}{=} \nu^{\prime} \circ \eta_{6} \circ \epsilon_{7} \circ \sigma_{15} \circ \Sigma^{19} \mu^{\prime} \stackrel{(19)}{=} 0 .
\end{gathered}
$$

Compondo com $\partial \omega(2)=\nu^{\prime} \circ \bar{\epsilon}_{6}$

$$
\begin{gathered}
\nu^{\prime} \circ \Sigma^{3}\left(\eta_{3} \circ \epsilon_{4}\right) \circ \Sigma^{12}\left(\nu^{\prime} \circ \bar{\epsilon}_{6}\right)=\nu^{\prime} \circ \eta_{6} \circ \epsilon_{7} \circ \Sigma^{12} \nu^{\prime} \circ \bar{\epsilon}_{18} \stackrel{(1)}{=} \nu^{\prime} \circ \eta_{6} \circ \Sigma^{4} \nu^{\prime} \circ \epsilon_{10} \circ \bar{\epsilon}_{18} \\
\stackrel{(4)}{=} \nu^{\prime} \circ \eta_{6} \circ 2 \nu_{7} \circ \epsilon_{10} \circ \bar{\epsilon}_{18}=2 \nu^{\prime} \circ \eta_{6} \circ \nu_{7} \circ \epsilon_{10} \circ \bar{\epsilon}_{18} \stackrel{(9)}{=} 0 .
\end{gathered}
$$

Compondo com $\partial \omega(2)=\eta_{3} \circ \bar{\mu}_{4}$

$$
\begin{gathered}
\nu^{\prime} \circ \Sigma^{3}\left(\eta_{3} \circ \epsilon_{4}\right) \circ \Sigma^{12}\left(\eta_{3} \circ \bar{\mu}_{4}\right)=\nu^{\prime} \circ \eta_{6} \circ \epsilon_{7} \circ \eta_{15} \circ \bar{\mu}_{16} \stackrel{(1)}{=} \nu^{\prime} \circ \eta_{6} \circ \eta_{7} \circ \epsilon_{8} \circ \bar{\mu}_{16} \\
\stackrel{(14)}{=} \nu^{\prime} \circ 4\left(\nu_{6} \circ \sigma_{9}\right) \circ \bar{\mu}_{16}=4 \nu^{\prime} \circ \nu_{6} \circ \sigma_{9} \circ \bar{\mu}_{16}=0 .
\end{gathered}
$$


Caso $k=23$. Temos

$$
\nu^{\prime} \circ \Sigma^{3} \partial \beta_{f}(2) \circ \Sigma^{12} \partial \omega(2)=0
$$

em $\pi_{34}\left(S^{3} ; 2\right)$. Aqui, $\partial \omega(2)$ está em $\pi_{22}\left(S^{3} ; 2\right)=\mathbb{Z} / 4\left[\bar{\mu}^{\prime}\right] \oplus \mathbb{Z} / 2\left[\nu^{\prime} \circ \mu_{6} \circ \sigma_{15}\right]$.

Compondo com $\partial \omega(2)=\bar{\mu}$

$$
\begin{aligned}
\nu^{\prime} \circ \Sigma^{3}\left(\eta_{3} \circ \epsilon_{4}\right) \circ \Sigma^{12}\left(\bar{\mu}^{\prime}\right) & =\nu^{\prime} \circ \eta_{6} \circ \epsilon_{7} \circ \Sigma^{12} \bar{\mu}^{\prime} \stackrel{(22)}{=} \nu^{\prime} \circ \eta_{6} \circ \epsilon_{7} \circ \sigma_{10}\left(2 \bar{\zeta}_{5}\right) \\
& =\nu^{\prime} \circ 2 \eta_{6} \circ \epsilon_{7} \circ \bar{\zeta}_{15}=0 .
\end{aligned}
$$

Compondo com $\partial \omega(2)=\nu^{\prime} \circ \mu_{6} \circ \sigma_{15}$

$$
\begin{gathered}
\nu^{\prime} \circ \Sigma^{3}\left(\eta_{3} \circ \epsilon_{4}\right) \circ \Sigma^{12}\left(\nu^{\prime} \circ \mu_{6} \circ \sigma_{15}\right)=\nu^{\prime} \circ \eta_{6} \circ \epsilon_{7} \circ \Sigma^{12} \nu^{\prime} \circ \mu_{18} \circ \sigma_{27} \\
\stackrel{(1)}{=} \nu^{\prime} \circ \eta_{6} \circ \Sigma^{4} \nu^{\prime} \circ \epsilon_{10} \circ \mu_{18} \circ \sigma_{27} \stackrel{(4)}{=} \nu^{\prime} \circ \eta_{6} \circ 2 \nu_{7} \circ \epsilon_{10} \circ \mu_{18} \circ \sigma_{27} \\
=2 \nu^{\prime} \circ \eta_{6} \circ \nu_{7} \circ \epsilon_{10} \circ \mu_{18} \circ \sigma_{27} \stackrel{(9)}{=} 0 .
\end{gathered}
$$

Caso $k=24$. Temos

$$
\nu^{\prime} \circ \Sigma^{3} \partial \beta_{f}(2) \circ \Sigma^{12} \partial \omega(2)=0
$$

em $\pi_{35}\left(S^{3} ; 2\right)$. Aqui, $\partial \omega(2)$ está em $\pi_{23}\left(S^{3} ; 2\right)=\mathbb{Z} / 4\left[\nu^{\prime} \circ \bar{\mu}_{6}\right] \oplus \mathbb{Z} / 2\left[\nu^{\prime} \circ \eta_{6} \circ \mu_{7} \circ \sigma_{16}\right]$ (este grupo pode ser encontrado em [32]).

Compondo com $\partial \omega(2)=\nu^{\prime} \circ \bar{\mu}_{6}$

$$
\begin{gathered}
\nu^{\prime} \circ \Sigma^{3}\left(\eta_{3} \circ \epsilon_{4}\right) \circ \Sigma^{12}\left(\nu^{\prime} \circ \bar{\mu}_{6}\right)=\nu^{\prime} \circ \eta_{6} \circ \epsilon_{7} \circ \Sigma^{12} \nu^{\prime} \circ \bar{\mu}_{18} \stackrel{(1)}{=} \nu^{\prime} \circ \eta_{6} \circ \Sigma^{4} \nu^{\prime} \circ \epsilon_{10} \circ \bar{\mu}_{18} \\
\stackrel{(4)}{=} \nu^{\prime} \circ \eta_{6} \circ 2 \nu_{7} \circ \epsilon_{10} \circ \bar{\mu}_{18}=2 \nu^{\prime} \circ \eta_{6} \circ \nu_{7} \circ \epsilon_{10} \circ \bar{\mu}_{18} \stackrel{(9)}{=} 0 .
\end{gathered}
$$

Compondo com $\partial \omega(2)=\nu^{\prime} \circ \eta_{6} \circ \mu_{7} \circ \sigma_{16}$

$$
\begin{gathered}
\nu^{\prime} \circ \Sigma^{3}\left(\eta_{3} \circ \epsilon_{4}\right) \circ \Sigma^{12}\left(\nu^{\prime} \circ \eta_{6} \circ \mu_{7} \circ \sigma_{16}\right)=\nu^{\prime} \circ \eta_{6} \circ \epsilon_{7} \circ \Sigma^{12} \nu^{\prime} \circ \eta_{18} \circ \mu_{19} \circ \sigma_{28} \\
\stackrel{(1)}{=} \nu^{\prime} \circ \eta_{6} \circ \Sigma^{4} \nu^{\prime} \circ \epsilon_{10} \circ \eta_{18} \circ \mu_{19} \circ \sigma_{28} \stackrel{(4)}{=} \nu^{\prime} \circ \eta_{6} \circ 2 \nu_{7} \circ \epsilon_{10} \circ \eta_{18} \circ \mu_{19} \circ \sigma_{28} \\
=2 \nu^{\prime} \circ \eta_{6} \circ \nu_{7} \circ \epsilon_{10} \circ \eta_{18} \circ \mu_{19} \circ \sigma_{28} \stackrel{(9)}{=} 0 .
\end{gathered}
$$

Caso $k=25$. Temos

$$
\nu^{\prime} \circ \Sigma^{3} \partial \beta_{f}(2) \circ \Sigma^{12} \partial \omega(2)=0
$$

em $\pi_{36}\left(S^{3} ; 2\right)$. Aqui, $\partial \omega(2)$ está em $\pi_{24}\left(S^{3} ; 2\right)=\mathbb{Z} / 2\left[\nu^{\prime} \circ \eta_{6} \circ \bar{\mu}_{7}\right]$ (este grupo pode ser encontrado em [30]). 
Compondo com $\partial \omega(2)=\nu^{\prime} \circ \eta_{6} \circ \bar{\mu}_{7}$

$$
\begin{gathered}
\nu^{\prime} \circ \Sigma^{3}\left(\eta_{3} \circ \epsilon_{4}\right) \circ \Sigma^{12}\left(\nu^{\prime} \circ \eta_{6} \circ \bar{\mu}_{7}\right)=\nu^{\prime} \circ \eta_{6} \circ \epsilon_{7} \circ \Sigma^{12} \nu^{\prime} \circ \eta_{18} \circ \bar{\mu}_{19} \\
\stackrel{(1)}{=} \nu^{\prime} \circ \eta_{6} \circ \Sigma^{4} \nu^{\prime} \circ \epsilon_{10} \circ \eta_{18} \circ \bar{\mu}_{19} \stackrel{(4)}{=} \nu^{\prime} \circ \eta_{6} \circ 2 \nu_{7} \circ \epsilon_{10} \circ \eta_{18} \circ \bar{\mu}_{19} \\
=2 \nu^{\prime} \circ \eta_{6} \circ \nu_{7} \circ \epsilon_{10} \circ \eta_{18} \circ \bar{\mu}_{19} \stackrel{(9)}{=} 0 .
\end{gathered}
$$

Caso $k=26$. Temos

$$
\nu^{\prime} \circ \Sigma^{3} \partial \beta_{f}(2) \circ \Sigma^{12} \partial \omega(2)=0
$$

em $\pi_{37}\left(S^{3} ; 2\right)$. Aqui, $\partial \omega(2)$ está em $\pi_{25}\left(S^{3} ; 2\right)=\mathbb{Z} / 2\left[\epsilon_{3} \circ \kappa_{11}\right]$ (este grupo pode ser encontrado em [30]).

Compondo com $\partial \omega(2)=\epsilon_{3} \circ \kappa_{11}$

$$
\begin{gathered}
\nu^{\prime} \circ \Sigma^{3}\left(\eta_{3} \circ \epsilon_{4}\right) \circ \Sigma^{12}\left(\epsilon_{3} \circ \kappa_{11}\right)=\nu^{\prime} \circ \eta_{6} \circ \epsilon_{7} \circ \epsilon_{15} \circ \kappa_{23} \stackrel{(23)}{=} \nu^{\prime} \circ \eta_{6} \circ \eta_{7} \circ \bar{\epsilon}_{8} \circ \kappa_{23} \\
\stackrel{(21)}{=} \nu^{\prime} \circ \Sigma^{3}\left(\eta_{3}^{2} \circ \bar{\epsilon}_{5}\right) \circ \kappa_{23}=\nu^{\prime} \circ \Sigma^{3}\left(2 \epsilon^{\prime}\right) \circ \kappa_{23}=2 \nu^{\prime} \circ \Sigma\left(\Sigma^{2} \epsilon^{\prime}\right) \circ \kappa_{23} \\
=4 \nu^{\prime} \circ \nu_{6} \circ \sigma_{9} \circ \kappa_{23} \stackrel{(14)}{=} 0 .
\end{gathered}
$$

As informações que temos quanto aos grupos de homotopia de $S^{3}$ nos permitem fazer o cálculo dessas composições até esse ponto, porém o que temos já nos faz suspeitar que os grupos de gauge $\mathcal{G}_{f_{(1,0)}}, \mathcal{G}_{f_{(1,1)}}$ têm o mesmo tipo. Na proposição que segue, vemos que é exatamente isso que ocorre.

Proposição 4.3.11. Os fibrados $\partial\left[f_{(1,0)}\right]=\mu_{3}$ e $\partial\left[f_{(1,1)}\right]=\mu_{3}+\eta_{3} \circ \epsilon_{4}$ possuem grupos de gauge com o mesmo tipo de homotopia ( logo, $\pi_{n}\left(\mathcal{G}_{f_{(1,0)}}\right) \approx \pi_{n}\left(\mathcal{G}_{f_{(1,1)}}\right)$ para qualquer valor de $n$ ).

Demonstração: Consideremos os grupos de gauge dos dois fibrados classificados pela classe das duas aplicações

$$
f_{(1,0)}, f_{(1,1)}: S^{13} \rightarrow B S^{3} .
$$

Sabemos que $\pi_{k}\left(\mathcal{G}_{f_{(1,0)}}\right) \approx \pi_{k}\left(\mathcal{G}_{f_{(1,1)}}\right)$, para todo $k \leq 26$. Sabemos também que os grupos de gauge desses dois fibrados podem apenas ser homotópos as fibras de homotopia $F_{f}$ e $F_{g}$ das duas aplicações

$$
f, g: S^{3} \rightarrow \Omega_{0}^{12} S^{3},
$$

tais que as aplicações adjuntas obtidas aplicando a lei exponencial

$$
a d: m_{0}\left(S^{3}, \Omega_{0}^{12} S^{3}\right) \rightarrow m_{0}\left(S^{15}, S^{3}\right)
$$

estão nas classes

$$
a d(f) \in \nu^{\prime} \circ \mu_{6} \in \pi_{15}\left(S^{3} ; 2\right)
$$


$\mathrm{e}$

$$
a d(g) \in \nu^{\prime} \circ\left(\mu_{6}+\eta_{6} \circ \epsilon_{7}\right) \in \pi_{15}\left(S^{3} ; 2\right) .
$$

Provemos que $F_{f} \sim F_{g}$, mostrando a existência de uma auto-equivalência de homotopia $s$ de $\Omega_{0}^{12} S^{3}$ tal que $g \sim s f$. A prova será dividida em vários passos.

Passo 0. Recordemos $\eta_{6} \circ \epsilon_{7}=\Sigma^{3}\left(\eta_{3} \circ \epsilon_{4}\right)$ e $\mu_{6}=\Sigma^{3} \mu_{3}$, por [46] pág. 39, 51, 56.

Passo 1. É melhor considerar o problema antes da restrição à componente da aplicação constante. A saber, consideremos as duas aplicações

$$
f, g: S^{3} \rightarrow \Omega_{0}^{12} S^{3}
$$

obtidas compondo com as equivalências de homotopia oportunas entre as componentes do espaço dos laços.

Passo 2. Mostremos que a imagem de $f$ e $g$ estão contidas em um subespaço de $\Omega_{0}^{12} S^{3}$ definido como segue (onde identificamos os representantes com as classes):

$$
\Lambda^{12} S^{3}=\left\{h_{x} \in \Omega_{0}^{12} S^{3} \mid h_{x}=\nu^{\prime} \circ\left(\iota_{3} \wedge \alpha\right)\left(x \wedge_{-}\right), x \in S^{3}, \alpha \in \Omega_{0}^{12} S^{3}\right\}
$$

De fato, se $h \in \operatorname{Im} f$, então

$$
\exists x \in S^{3} \mid h=h_{x}=f(x),
$$

e, para todo $y \in S^{12}$,

$$
h_{x}: S^{3} \wedge S^{12} \stackrel{\iota_{3} \wedge \alpha}{\longrightarrow} S^{3} \wedge S^{3} \stackrel{\nu^{\prime}}{\longrightarrow} S^{3}
$$

e

$$
h_{x}(y)=f(x)(y)=a d(f)(x \wedge y)=\nu^{\prime} \circ \mu_{6}(x \wedge y)=\nu^{\prime} \circ\left(\iota_{3} \wedge \mu_{3}\right)(x \wedge y),
$$

e a tese segue. O mesmo argumento funciona para $g$. O possível $\alpha$ é, por conseguinte, $\mu_{3}$ ou $\mu_{3}+\eta_{3} \circ \epsilon_{4}$. No próximo passo introduziremos alguma informação sobre essas duas classes.

Logo, podemos reduzir o problema considerando as duas aplicações:

$$
f, g: S^{3} \rightarrow \Lambda^{12} S^{3}
$$

e precisamos encontrar uma auto-equivalência de homotopia de $\Lambda^{12} S^{3}$.

Passo 3. Recordemos a construção das classes $\mu_{3}$ e $\epsilon_{3}$. Construímos um espaço $K$ pelo push out

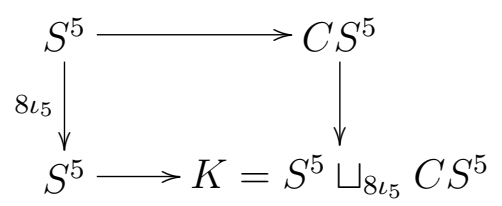


Também, $\Sigma\left(A \sqcup_{\alpha} C B\right)=\Sigma A \sqcup_{\Sigma \alpha} C \Sigma B$. Do fato que $\nu^{\prime} \circ 8 \iota_{6}=8 \nu^{\prime}=0$, temos uma extensão $\bar{\nu}^{\prime}$ como no diagrama

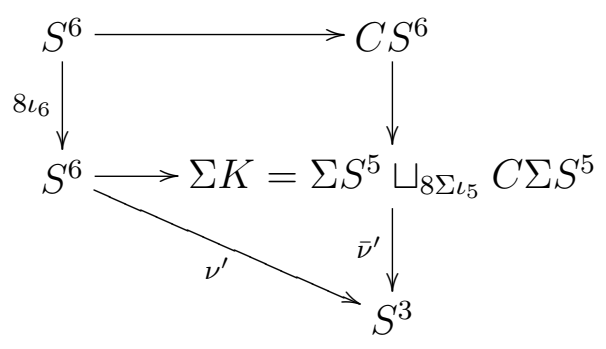

Como $\nu^{\prime} \circ \nu_{6}=0$ [46] (Prop. 5.11 pág. 46), temos uma co-extensão $\tilde{\nu}_{6}$

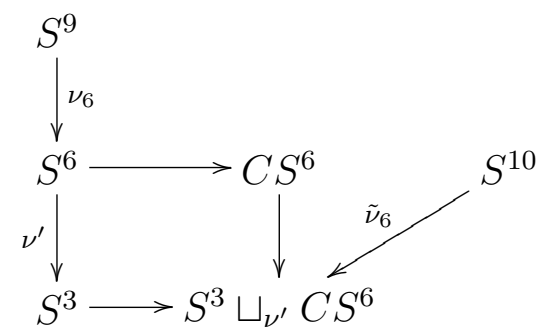

O elemento $\epsilon_{3}$ é o único elemento da composição secundária

$$
\left\{\epsilon_{3}\right\}=\left\{\eta_{3}, \Sigma \nu^{\prime}, \Sigma \nu_{6}\right\}_{1} \subseteq \pi_{11}\left(S^{3} ; 2\right)
$$

que é definido pelo diagrama abaixo, já que $\eta_{3} \circ \Sigma \nu^{\prime}=\nu^{\prime} \circ \nu_{6}=0$ ([46] Lema 5.7 pág. 43 e Prop. 5.11 pág. 46).

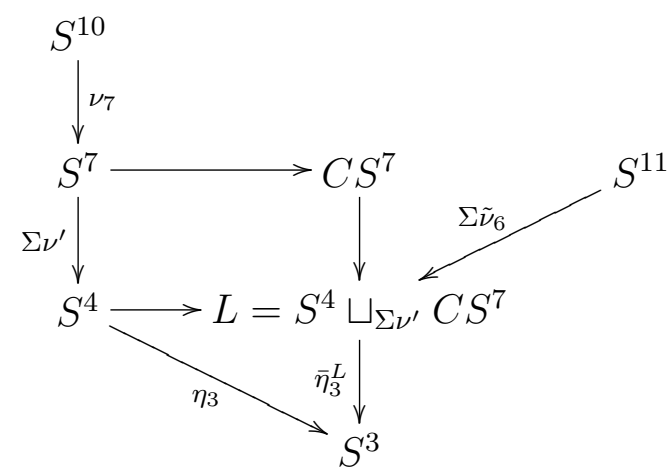

Logo, é uma composição

$$
\epsilon_{3}=\bar{\eta}_{3}^{L} \circ \Sigma \tilde{\nu}_{6}
$$

Agora, do fato que $8 \iota_{5} \circ \nu_{5}=0$, temos uma co-extensão $\tilde{\nu}_{5}$

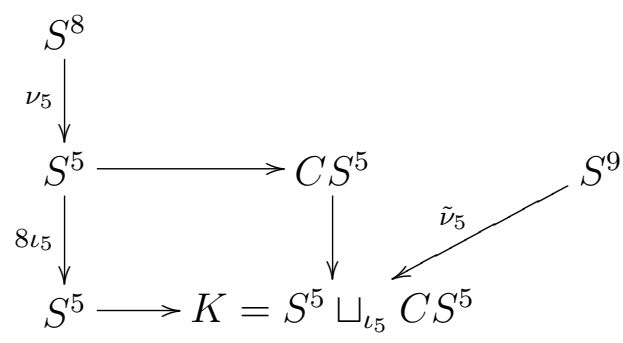


Como $\eta_{3} \circ \Sigma \bar{\nu}^{\prime}=\bar{\nu}^{\prime} \circ \Sigma \tilde{\nu}_{5}=0$ ([46] pág. 55, 56), podemos construir a composição secundária

$$
\left\{\eta_{3}, \Sigma \bar{\nu}^{\prime}, \Sigma^{2} \tilde{\nu}_{5}\right\}_{1} \subseteq \pi_{12}\left(S^{3} ; 2\right)
$$

com o diagrama

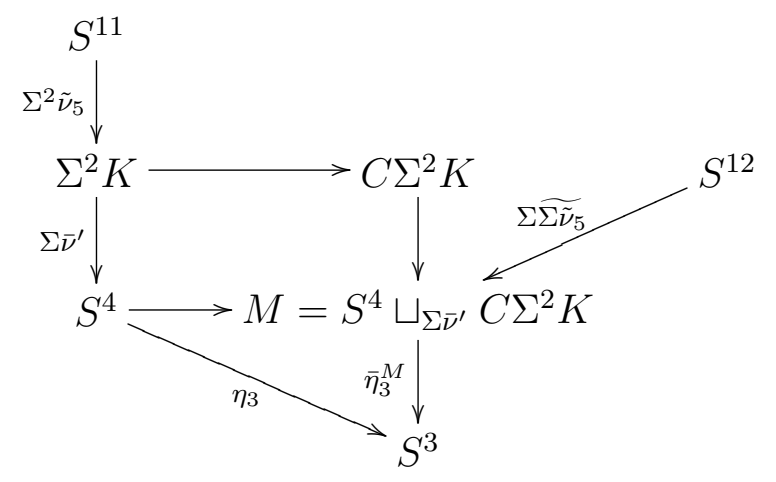

e

$$
\mu_{3} \in\left\{\eta_{3}, \Sigma \bar{\nu}^{\prime}, \Sigma^{2} \tilde{\nu}_{5}\right\}_{1} \subseteq \pi_{12}\left(S^{3} ; 2\right)
$$

é uma composição

$$
\mu_{3}=\bar{\eta}_{3}^{M} \circ \Sigma \tilde{\gamma}
$$

denotando por $\Sigma \tilde{\gamma}$ o elemento $\Sigma \widetilde{\Sigma \tilde{\nu}_{5}}$.

Observe que o complexo $L$ é um subcomplexo de $M$, e denotamos a inclusão por $j: L \rightarrow M$.

Passo 4. Usando as construções descritas no Passo 3, e observando que

$$
\epsilon_{3} \circ \eta_{11}=\bar{\eta}_{3}^{L} \circ \Sigma \tilde{\nu}_{6} \circ \eta_{11}=\bar{\eta}_{3}^{M} \circ j_{*} \Sigma \tilde{\nu}_{6} \circ \eta_{11},
$$

vemos que as aplicações $\alpha$ aparecendo na decomposição das aplicações $h$, introduzidas no Passo 2 nas imagens de $f$ e $g$, são da seguinte forma: $\alpha=\mu_{3}=\bar{\eta}_{3}^{M} \circ \Sigma \tilde{\gamma}$ ou $\alpha=$ $\mu_{3}+\epsilon_{3} \circ \eta_{11}=\bar{\eta}_{3}^{M} \circ \Sigma \tilde{\gamma}+\bar{\eta}_{3}^{M} \circ j_{*} \Sigma \tilde{\nu}_{6} \circ \eta_{11}=\bar{\eta}_{3}^{M} \circ\left(\Sigma \tilde{\gamma}+j_{*} \Sigma \tilde{\nu}_{6} \circ \eta_{11}\right)$, e isso significa que todas as aplicações na imagem de $f$ e $g$ têm a forma:

$$
\begin{aligned}
h_{x} & =\nu^{\prime} \circ\left(\iota_{3} \wedge \mu_{3}\right)\left(x \wedge{ }_{-}\right)=\nu^{\prime} \circ\left(\iota_{3} \wedge \bar{\eta}_{3}^{M} \circ \Sigma \tilde{\gamma}\right)\left(x \wedge_{-}\right) \\
& =\nu^{\prime} \circ\left(\iota_{3} \wedge \bar{\eta}_{3}^{M}\right) \circ\left(\iota_{3} \wedge \Sigma \tilde{\gamma}\right)\left(x \wedge \wedge_{-}\right), \\
h_{x} & =\nu^{\prime} \circ\left(\iota_{3} \wedge\left(\mu_{3}+\epsilon_{3} \circ \eta_{11}\right)\right)\left(x \wedge{ }_{-}\right)=\nu^{\prime} \circ\left(\iota_{3} \wedge \bar{\eta}_{3}^{M} \circ\left(\Sigma \tilde{\gamma}+j_{*} \Sigma \tilde{\nu}_{6} \circ \eta_{11}\right)\right)\left(x \wedge \_\right) \\
& =\nu^{\prime} \circ\left(\iota_{3} \wedge \bar{\eta}_{3}^{M}\right) \circ\left(\iota_{3} \wedge\left(\Sigma \tilde{\gamma}+j_{*} \Sigma \tilde{\nu}_{6} \circ \eta_{11}\right)\right)\left(x \wedge \wedge_{-}\right) .
\end{aligned}
$$

Assim, todas essas aplicações se fatoram através do espaço $M$ como segue:

$$
h_{x}: x \wedge S^{12 \stackrel{\iota_{3} \wedge \Sigma \tilde{\gamma}}{\longrightarrow}} x \wedge M \stackrel{\iota_{3} \wedge \bar{\eta}_{3}^{M}}{\longrightarrow} x \wedge S^{3} \stackrel{\nu^{\prime}}{\longrightarrow} S^{3}
$$

para algum $\Sigma \tilde{\gamma}$ como descrito acima, $x \in S^{3}$.

Denotamos por $L^{12} S^{3}$ o espaço

$$
L^{12} S^{3}=\left\{h_{x} \in \Omega^{12} S^{3} \mid h_{x}=\nu^{\prime} \circ\left(\iota_{3} \wedge \bar{\eta}_{3}^{M}\right) \circ\left(\iota_{3} \wedge \Sigma \tilde{\gamma}\right)\left(x \wedge \wedge_{-}\right), x \in S^{3}, \Sigma \tilde{\gamma} \in \Omega^{12} M\right\}
$$


Vimos que

$$
f, g: S^{3} \rightarrow L^{12} S^{3}
$$

e assim precisamos encontrar uma auto-equivalência de homotopia $s$ de $L^{12} S^{3}$, tal que $g=s f$.

Mais precisamente, denotando por $\Sigma \tilde{\gamma}_{1}=\Sigma \widetilde{\Sigma \tilde{\nu}_{5}}$ e por $\Sigma \gamma_{2}=j_{*} \Sigma \tilde{\nu}_{6} \circ \eta_{11}$ em $\Omega^{12}(M)$, temos

$$
\begin{aligned}
& \operatorname{Im} f=\bigcup_{x \in S^{3}}\left\{h_{x} \in \Omega^{12} S^{3} \mid h_{x}=\nu^{\prime} \circ\left(\iota_{3} \wedge \bar{\eta}_{3}^{M}\right) \circ\left(\iota_{3} \wedge \Sigma \tilde{\gamma}_{1}\right)\left(x \wedge{ }_{-}\right)\right\} \\
& \operatorname{Im} g=\bigcup_{x \in S^{3}}\left\{h_{x} \in \Omega^{12} S^{3} \mid h_{x}=\nu^{\prime} \circ\left(\iota_{3} \wedge \bar{\eta}_{3}^{M}\right) \circ\left(\iota_{3} \wedge\left(\Sigma \tilde{\gamma}_{1}+\Sigma \gamma_{2}\right)\right)\left(x \wedge \_\right)\right\} .
\end{aligned}
$$

Em outras palavras, as aplicações $f$ e $g$ agem como segue

$$
\begin{aligned}
& f: x \mapsto h_{x}=\nu^{\prime} \circ\left(\iota_{3} \wedge \bar{\eta}_{3}^{M}\right) \circ\left(\iota_{3} \wedge \Sigma \tilde{\gamma}_{1}\right)\left(x \wedge{ }_{-}\right), \\
& g: x \mapsto h_{x}=\nu^{\prime} \circ\left(\iota_{3} \wedge \bar{\eta}_{3}^{M}\right) \circ\left(\iota_{3} \wedge\left(\Sigma \tilde{\gamma}_{1}+\Sigma \gamma_{2}\right)\right)\left(x \wedge_{-}\right) .
\end{aligned}
$$

Observação 4.3.12. Uma forma mais explícita dessas aplicações é possível recordando que $\nu^{\prime}(x \wedge y)=x y x^{-1}$, na componente da identidade de $\Omega^{3} S^{3}$.

Passo 5. Cálculos da Homologia. Encontremos algumas informações sobre a topologia dos espaços $K, L$ e $M$, definidos no Passo 3. Recordemos as definições $K=S^{5} \sqcup_{8 \iota_{5}} C S^{5}$, $L=S^{4} \sqcup_{\Sigma \nu^{\prime}} C S^{7}, M=S^{4} \sqcup_{\Sigma \bar{\nu}^{\prime}} C \Sigma^{2} K$, com $\Sigma^{2} K=S^{7} \sqcup_{8 \iota 7} C S^{7}$. Assim, $\Sigma^{2} K$ é um complexo com uma célula em dimensões 0,7 e $8, L$ é um complexo com uma célula em dimensões 0,4 e 8, e $M$ é um complexo com 4-esqueleto $S^{4}$ e uma célula em dimensão 9. Agora, $S^{7}$ é um retrato de deformação de um conjunto aberto em $\Sigma^{2} K$. Apenas considere um pequeno colar no cone colado, então (veja por exemplo [35] ex. 2, pág. $230), H_{p}\left(\Sigma^{2} K, S^{7} ; \mathbb{Z}\right) \approx H_{p}\left(\Sigma^{2} K / S^{7} ; \mathbb{Z}\right)$. Temos $\Sigma^{2} K / S^{7} \sim S^{8}$ (use, por exemplo, a seqüência de Puppe). Logo,

$$
H_{p}\left(\Sigma^{2} K, S^{7} ; \mathbb{Z}\right) \approx H_{p}\left(\Sigma^{2} K / S^{7} ; \mathbb{Z}\right) \approx H_{q}\left(S^{8} ; \mathbb{Z}\right)
$$

Pelo mesmo argumento,

$$
\begin{aligned}
H_{p}\left(L, S^{4} ; \mathbb{Z}\right) & \approx H_{p}\left(L / S^{4} ; \mathbb{Z}\right) \approx H_{q}\left(S^{8} ; \mathbb{Z}\right), \\
H_{p}\left(M, S^{4} ; \mathbb{Z}\right) & \approx H_{p}\left(M / S^{4} ; \mathbb{Z}\right) \approx H_{q}\left(\Sigma^{3} K ; \mathbb{Z}\right) \approx H_{q-1}\left(\Sigma^{2} K ; \mathbb{Z}\right) .
\end{aligned}
$$

Agora, uma análise direta da construção geométrica mostra que $M / L \sim S^{9}$ e, como podemos obter o primeiro quociente considerando o quociente $M / S^{4} \sim \Sigma \Sigma^{2} K$, e então considerando o quociente com o subcomplexo $C S^{7}$ contido na base da suspensão, aplicamos o mesmo argumento usado acima e obtemos

$$
H_{p}(M, L ; \mathbb{Z}) \approx H_{p}(M / L ; \mathbb{Z}) \approx H_{q}\left(S^{9} ; \mathbb{Z}\right)
$$

Calculamos, primeiro, os grupos de homologia e homotopia de $\Sigma^{2} K$. Da seqüência exata de homologia do par, $i: S^{7} \rightarrow \Sigma^{2} K$ (todos os coeficientes em $\mathbb{Z}$ ), temos 


$$
H_{q}\left(S^{7}\right) \stackrel{i_{*}}{\longrightarrow} H_{q}\left(\Sigma^{2} K\right) \longrightarrow H_{q}\left(\Sigma^{2} K, S^{7}\right) \approx H_{q}\left(S^{8}\right) \stackrel{\partial}{\longrightarrow} H_{q}\left(S^{7}\right) .
$$

Para $0<q<7$

$$
0 \stackrel{i_{*}}{\longrightarrow} H_{q}\left(\Sigma^{2} K\right) \longrightarrow 0,
$$

e $\operatorname{assim} H_{q}\left(\Sigma^{2} K\right)=H_{q}\left(\Sigma^{2} K, S^{7}\right)=0$.

$$
\begin{gathered}
H_{8}\left(S^{7}\right) \stackrel{i_{*}}{\longrightarrow} H_{8}\left(\Sigma^{2} K\right) \longrightarrow H_{8}\left(\Sigma^{2} K, S^{7}\right) \approx H_{8}\left(S^{8}\right) \stackrel{\partial}{\longrightarrow} H_{7}\left(S^{7}\right) \stackrel{i_{*}}{\longrightarrow} \\
\stackrel{i_{*}}{\longrightarrow} H_{7}\left(\Sigma^{2} K\right) \longrightarrow H_{7}\left(\Sigma^{2} K, S^{7}\right) \stackrel{\longrightarrow}{\longrightarrow} H_{6}\left(S^{7}\right),
\end{gathered}
$$

como $\partial$ é a multiplicação por 8

$$
0 \stackrel{i_{*}}{\longrightarrow} H_{8}\left(\Sigma^{2} K\right) \longrightarrow \mathbb{Z} \stackrel{8}{\longrightarrow} \mathbb{Z} \stackrel{i_{*}}{\longrightarrow} H_{7}\left(\Sigma^{2} K\right) \longrightarrow 0 .
$$

Logo, os únicos grupos de homologia não triviais são

$$
\begin{gathered}
H_{7}\left(\Sigma^{2} K\right) \approx \mathbb{Z} / 8, \\
H_{8}\left(\Sigma^{2} K, S^{7}\right) \approx \mathbb{Z} .
\end{gathered}
$$

Segue de Hurewicz ([47] (7.8) e (7.10) pág. 180) que

$$
\begin{aligned}
& \pi_{7}\left(\Sigma^{2} K\right) \approx \mathbb{Z} / 8, \\
& \pi_{8}\left(\Sigma^{2} K, S^{7}\right) \approx \mathbb{Z} .
\end{aligned}
$$

Calculemos, agora, os grupos de homologia e homotopia de $L$. Considereremos a seqüência de homologia do par $i: S^{4} \rightarrow L$ e recordemos que $H_{q}\left(L, S^{4} ; \mathbb{Z}\right) \approx H_{q}\left(S^{8} ; \mathbb{Z}\right)$.

$$
H_{q}\left(S^{4}\right) \stackrel{i_{*}}{\longrightarrow} H_{q}(L) \longrightarrow H_{q}\left(L, S^{4}\right) \approx H_{q}\left(S^{8}\right) \stackrel{\partial}{\longrightarrow} H_{q-1}\left(S^{4}\right) .
$$

Para $0<q<4, H_{q}(L) \approx H_{q}\left(L, S^{4}\right)=0$.

Para $q=4, H_{4}(L) \approx \mathbb{Z}, H_{4}\left(L, S^{4}\right)=0$.

Para $q=5,6,7, H_{q}(L)=0, H_{q}\left(L, S^{4}\right)=0$.

$$
\begin{aligned}
H_{9}\left(S^{4}\right) \stackrel{i_{*}}{\longrightarrow} H_{9}(L) \longrightarrow H_{9}\left(L, S^{4}\right) \approx H_{9}\left(S^{8}\right) \stackrel{\partial}{\longrightarrow} \\
\stackrel{\partial}{\longrightarrow} H_{8}\left(S^{4}\right) \stackrel{i_{*}}{\longrightarrow} H_{8}(L) \longrightarrow H_{8}\left(L, S^{4}\right) \approx H_{8}\left(S^{8}\right) \longrightarrow H_{7}\left(S^{4}\right),
\end{aligned}
$$

obtendo

$$
0 \stackrel{i_{*}}{\longrightarrow} H_{9}(L) \longrightarrow 0 \stackrel{\partial}{\longrightarrow} 0 \stackrel{i_{*}}{\longrightarrow} H_{8}(L) \longrightarrow \mathbb{Z} \longrightarrow 0 .
$$

Assim, $H_{8}(L) \approx H_{8}\left(L, S^{4}\right) \approx \mathbb{Z}$ e o grupos em dimensões maiores são nulos. Novamente, pelo Toerema de Hurewicz

$$
\pi_{4}(L) \approx H_{4}(L) \approx \mathbb{Z}
$$




$$
\pi_{8}\left(L, S^{4}\right) \approx H_{8}\left(L, S^{4}\right) \approx \mathbb{Z}
$$

Também precisamos algumas informações sobre a co-homologia. Pelo Teorema dos coeficientes universais ([35] 53.1):

$$
H^{q}(M) \approx \operatorname{Hom}\left(H_{q}(M), \mathbb{Z}\right) \oplus \operatorname{Ext}\left(H_{q-1}(M), \mathbb{Z}\right)
$$

Então

$$
\begin{aligned}
& H^{q}(L)=0, \quad 0<q<4, \\
& H^{4}(L) \approx \operatorname{Hom}(\mathbb{Z}, \mathbb{Z}) \oplus \operatorname{Ext}(0, \mathbb{Z}) \approx \mathbb{Z}, \\
& H^{5}(L) \approx \operatorname{Hom}(0, \mathbb{Z}) \oplus \operatorname{Ext}(\mathbb{Z}, \mathbb{Z})=0, \\
& H^{q}(L)=0, \quad q=6,7, \\
& H^{8}(L) \approx \operatorname{Hom}(\mathbb{Z}, \mathbb{Z}) \oplus \operatorname{Ext}(0, \mathbb{Z}) \approx \mathbb{Z}, \\
& H^{9}(L) \approx \operatorname{Hom}(0, \mathbb{Z}) \oplus \operatorname{Ext}(\mathbb{Z}, \mathbb{Z})=0 .
\end{aligned}
$$

Logo, se $h: L \rightarrow L$ é uma aplicação, o homomorfismo induzido $h^{*}$ é um homomorfismo de anel na co-homologia. A saber, se $H^{4}(L)=\mathbb{Z}[x]$, com um gerador fixado $x$, então $h^{*}\left(x^{2}\right)=\left(h^{*}(x)\right)^{2}$. Mas observe que $x^{2}$ não é um gerador de $H^{8}(L)$. De fato, se $y$ é tal gerador, temos a relação $x^{2}=H\left(\Sigma \nu^{\prime}\right) y$, sendo $H(f)$ o invariante de Hopf de $f$ (veja [7]). Agora, $H\left(\Sigma \nu^{\prime}\right)=0$, já que $H \Sigma=0$ ([46] [17]).

Continuando, calculemos os grupos de $M$. Consideremos a seqüência de homologia do $\operatorname{par}\left(M, S^{4}\right)$ e seja $i: S^{4} \rightarrow M$ a inclusão. Recordemos que $H_{q}\left(M, S^{4} ; \mathbb{Z}\right) \approx H_{q-1}\left(\Sigma^{2} K ; \mathbb{Z}\right)$.

$$
H_{q}\left(S^{4}\right) \stackrel{i_{*}}{\longrightarrow} H_{q}(M) \longrightarrow H_{q}\left(M, S^{4}\right) \approx H_{q-1}\left(\Sigma^{2} K\right) \stackrel{\partial}{\longrightarrow} H_{q-1}\left(S^{4}\right) .
$$

Para $0<q<4, H_{q}(M) \approx H_{q}\left(M, S^{4}\right)=0$.

Para $q=4, H_{4}(M) \approx \mathbb{Z}, H_{4}\left(M, S^{4}\right)=0$.

Para $q=5,6,7 H_{q}(M)=0, H_{q}\left(M, S^{4}\right)=0$.

$$
\begin{gathered}
H_{9}\left(S^{4}\right) \stackrel{i_{*}}{\longrightarrow} H_{9}(M) \longrightarrow H_{9}\left(M, S^{4}\right) \approx H_{8}\left(\Sigma^{2} K\right) \stackrel{\partial}{\longrightarrow} \\
\stackrel{\partial}{\longrightarrow} H_{8}\left(S^{4}\right) \stackrel{i_{*}}{\longrightarrow} H_{8}(M) \longrightarrow H_{8}\left(M, S^{4}\right) \approx H_{7}\left(\Sigma^{2} K\right) \longrightarrow H_{8}\left(S^{4}\right),
\end{gathered}
$$

obtendo

$$
0 \stackrel{i_{*}}{\longrightarrow} H_{9}(M) \longrightarrow 0 \stackrel{\partial}{\longrightarrow} 0 \stackrel{i_{*}}{\longrightarrow} H_{8}(M) \longrightarrow \mathbb{Z} / 8 \longrightarrow 0 .
$$

Assim, $H_{8}(M) \approx H_{8}\left(M, S^{4}\right) \approx \mathbb{Z} / 8$, e os grupos maiores são nulos. Pelo Teorema de Hurewicz

$$
\begin{gathered}
\pi_{4}(M) \approx H_{4}(M) \approx \mathbb{Z} \\
\pi_{8}\left(M, S^{4}\right) \approx H_{8}\left(M, S^{4}\right) \approx \mathbb{Z} / 8 .
\end{gathered}
$$


Passo 6. Considereremos o seguinte diagrama

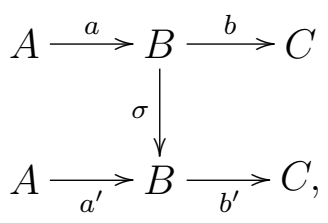

no qual $\sigma$ é uma equivalência de homotopia. Suponha que $f=b a, g=b^{\prime} a^{\prime}$ e $a^{\prime}=\sigma a$. O espaço-produto $m_{0}(A, B) \times m_{0}(B, C)$ é um subespaço do espaço das aplicações baseadas $m_{0}(A, C)$ e coincide com o subespaço das aplicações que se fatoram através de $B$, o qual denotamos por $m_{0}(A, B, C)$. A equivalência $\sigma$ define duas equivalências:

$$
\begin{aligned}
\sigma_{\#}: m_{0}(A, B) \rightarrow m_{0}(A, B), & \sigma_{\#}(a)=\sigma a, \\
\sigma^{\#}: m_{0}(B, C) \rightarrow m_{0}(B, C), & \sigma^{\#}\left(b^{\prime}\right)=b^{\prime} \sigma .
\end{aligned}
$$

A primeira define uma equivalência

$$
\sigma_{\#}: m_{0}(A, B, C) \rightarrow m_{0}(A, B, C), \quad \sigma_{\#}(f=a b)=b \sigma a,
$$

para cada $b$ fixado; a segunda define uma equivalência

$$
\sigma^{\#}: m_{0}(A, B, C) \rightarrow m_{0}(A, B, C), \quad \sigma^{\#}(f=a b)=b \sigma a,
$$

para cada $a$ fixado.

Passo 7. Calculemos alguns grupos de homotopia de $K, \Sigma^{3} K$ e $M$. Pelo Teorema de suspensão de Freudenthal ([47] VII.7.13), como $\Sigma^{2} K$ é $(7-1)$-conexo, $\Sigma: \pi_{q}(K) \rightarrow$ $\pi_{q+1}(\Sigma K)$ é um isomorfismo para $q<(10-1)=9$. Então $\Sigma^{3} K$ é $(8-1)$-conexo.

Seja $i: S^{4} \rightarrow M$ a inclusão e $\kappa: M \rightarrow M / S^{4}$ a projeção. O par $\left(M, S^{4}\right)$ é (8-1)-conexo já que $M / S^{4} \sim \Sigma^{3} K$ (veja os cálculos da homologia no Passo 5). Logo, $\pi_{q}\left(M, S^{4}\right) \approx$ $\pi_{q}\left(\Sigma^{3} K\right)$, para $q<(8+4-1)=11$, por Blackers-Massey ([47] VII.7.12), com o isomorfismo induzido pela projeção. Usando a seqüência de homotopia do par com $q<11$ :

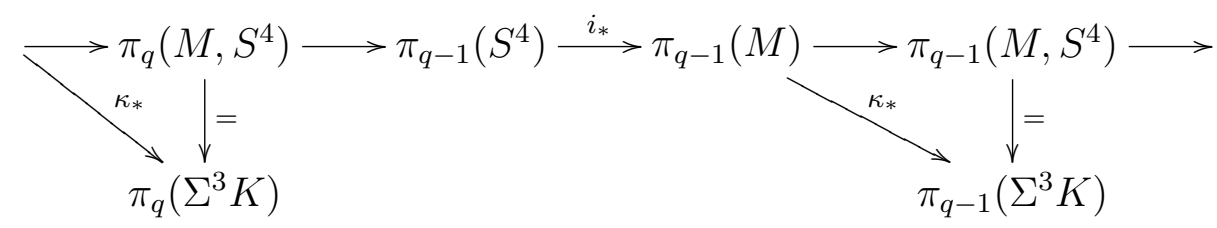

obtemos

$$
\longrightarrow \pi_{q}\left(\Sigma^{3} K\right) \longrightarrow \pi_{q-1}\left(S^{4}\right) \stackrel{i_{*}}{\longrightarrow} \pi_{q-1}(M) \stackrel{\kappa_{*}}{\longrightarrow} \pi_{q-1}\left(\Sigma^{3} K\right) \longrightarrow
$$

Com $q=5$ e $q=6$, isso mostra que $\pi_{4}(M)=\mathbb{Z}\left[i_{*}\left(\iota_{4}\right)\right], \pi_{5}(M)=\mathbb{Z}\left[i_{*}\left(\eta_{4}\right)\right]$.

Passo 8. Pelo Passo 6, para encontrar uma equivalência do espaço $L^{12} S^{3}$ definido no final do Passo 4, é suficiente encontrar uma equivalência $\sigma$ de $M$, já que disso temos o diagrama 


$$
\begin{aligned}
& S^{3} \wedge S^{12} \stackrel{\iota_{3} \wedge \Sigma \tilde{\gamma}}{\longrightarrow} S^{3} \wedge M \stackrel{\iota_{3} \wedge \bar{\eta}_{3}^{M}}{\longrightarrow} S^{3} \wedge S^{3} \stackrel{\nu^{\prime}}{\longrightarrow} S^{3} \\
& \iota_{3} \wedge \sigma \downarrow \\
& S^{3} \wedge M
\end{aligned}
$$

o qual permite aplicar a construção feita no Passo 6.

Construimos uma auto-equivalência de $M$. Consideremos a seqüência de Puppe do push out:

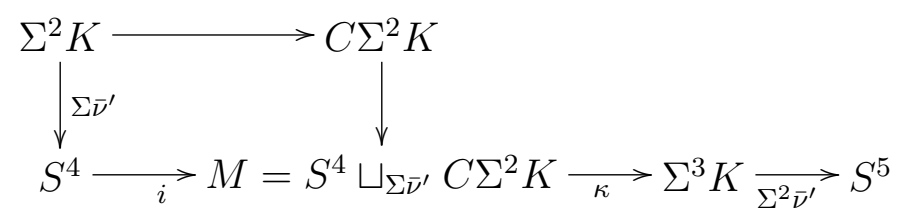

Obtendo a seqüência co-exata dos conjuntos e grupos (veja [42] pág. 366):

$$
\longrightarrow \pi_{5}(M) \stackrel{\left(\Sigma^{2} \bar{\nu}^{\prime}\right)}{\longrightarrow}\left[\Sigma^{3} K, M\right]_{0} \stackrel{\kappa^{*}}{\longrightarrow}[M, M]_{0} \stackrel{i^{*}}{\longrightarrow} \pi_{4}(M) \stackrel{\left(\Sigma \bar{\nu}^{\prime}\right)}{\longrightarrow}\left[\Sigma^{2} K, M\right]_{0} .
$$

Vimos que $\pi_{4}(M) \approx \mathbb{Z}$, e é gerado pela classe da inclusão $[i]$. Também, pela definição do push out, $i \Sigma \bar{\nu}^{\prime} \sim 0$. Como $\Sigma \bar{\nu}^{\prime}$ é uma suspensão, $\left(\sum \bar{\nu}^{\prime}\right)^{*}(k[i])=k\left(\sum \bar{\nu}^{\prime}\right)^{*}([i])$, logo deduzimos que o homomorfismo $\left(\Sigma \bar{\nu}^{\prime}\right)^{*}$ é o trivial (já que ele aplica o gerador em 0 ). Assim,

$$
\longrightarrow \pi_{5}(M) \stackrel{\left(\Sigma^{2} \bar{\nu}^{\prime}\right)}{\longrightarrow}\left[\Sigma^{3} K, M\right]_{0} \stackrel{p^{*}}{\longrightarrow}[M, M]_{0} \stackrel{i^{*}}{\longrightarrow} \pi_{4}(M) \longrightarrow 0 .
$$

Aplicando a mesma construção com $S^{4}$ e $\Sigma^{3} K$, respectivamente, obtemos o diagrama comutativo (as linhas verticais não são necessariamente exatas):

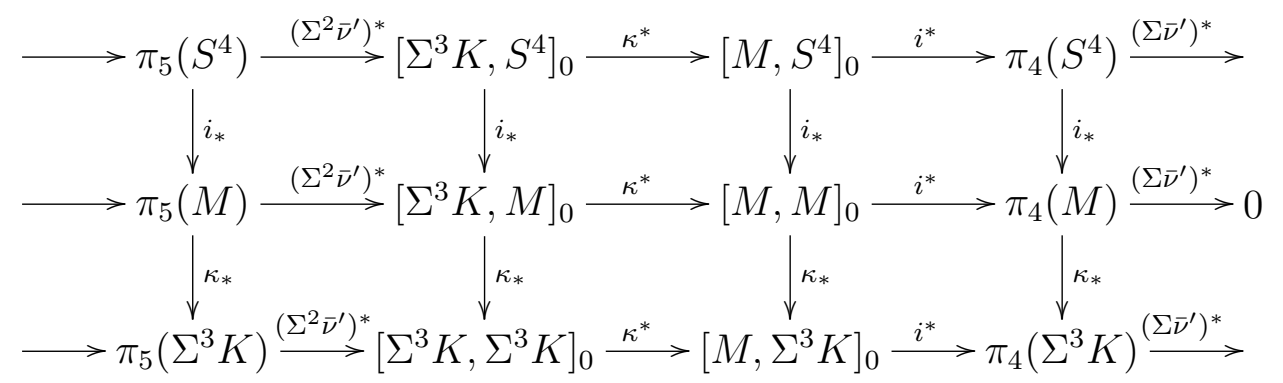

Temos mais informações desse diagrama que não serão necessárias. Usando os cálculos do Passo \%, o diagrama se torna

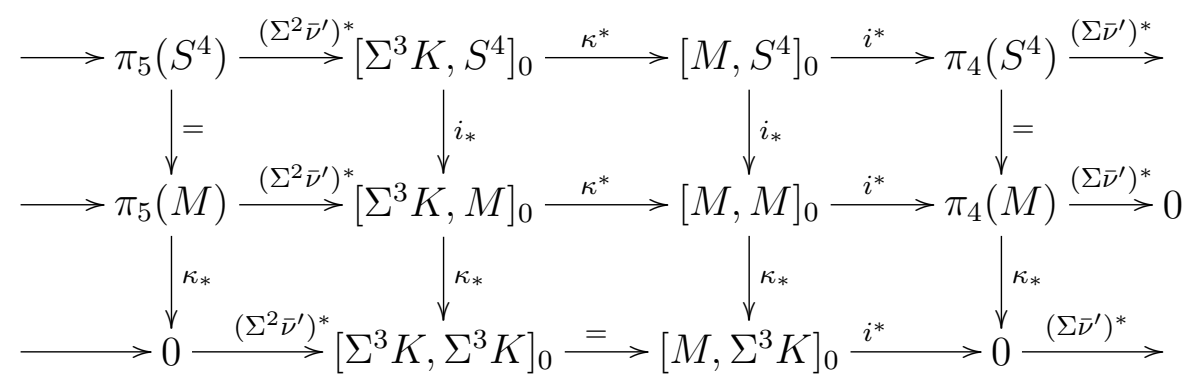

Também, como $i^{*}:[M, M]_{0} \rightarrow \pi_{4}(M)$ é sobrejetiva, para cada aplicação $k i_{4} \in \pi_{4}(M)$, existe uma aplicação $\sigma_{k} \in[M, M]_{0}$, na qual $k \in \mathbb{Z}$ vem do fato de que as aplicações $k i_{4}$ são 
enumeradas pela composição de uma auto-aplicação $k \iota_{4}$ com a inclusão $i: S^{4} \rightarrow M$, isto é, $k i_{4}=i_{*}\left(k \iota_{4}\right)=k i_{*} \iota_{4}$. Em particular, $\sigma_{1}=1$ é a identidade, sendo uma auto-equivalência de homotopia. Mas também $\sigma=\sigma_{-1}$ é uma auto-equivalência.

Podemos produzir auto-equivalências de homotopia de $M$ usando a abordagem feita em [36]. Temos homomorfismos

$$
\lambda: x \mapsto 1^{x}
$$

e

$$
\pi: x \rightarrow 1+k_{*}(x),
$$

fazendo o seguinte diagrama comutar

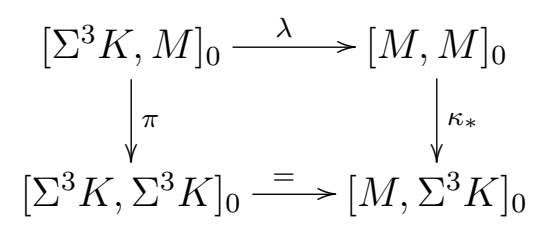

Aqui $1^{x}=\nabla(1 \vee x) \Delta$ é aplicação definida pela co-ação de Hilton,

$$
1^{x}: M \stackrel{\theta}{\longrightarrow} M \vee \Sigma^{3} K \stackrel{1 \vee x}{\longrightarrow} M \vee M \stackrel{\nabla}{\longrightarrow} M .
$$

Sabemos por [36] que $\lambda \pi^{-1}(1)$ é um subgrupo do grupo das auto-equivalências de $M$.

Construímos uma aplicação $x: \Sigma^{3} K \rightarrow M$, tal que $\pi(x)=1$. Isso significa que $\lambda(x)=1^{x}$ é uma auto-equivalência de $M$.

Consideremos o push out

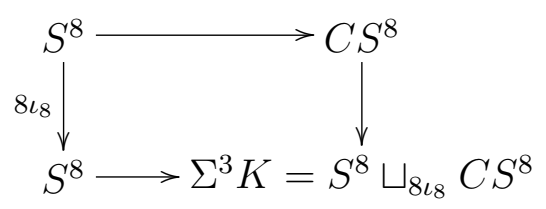

Como $\nu_{5} \circ 8 \iota_{8}=0$, temos extensões $\bar{\nu}_{5}$ de $\nu_{5}$ como segue

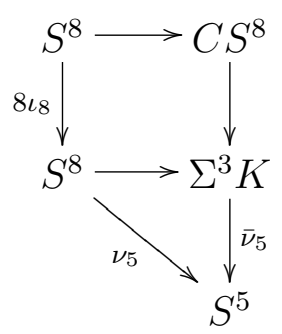

Já que $\Sigma^{3} K / S^{8}=S^{9}$, do push out

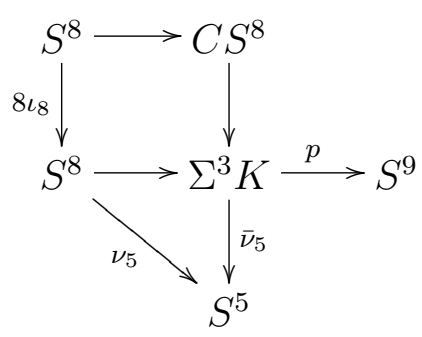


obtemos a seqüência de Puppe

$$
\pi_{9}\left(S^{5}\right) \stackrel{8}{\longrightarrow} \pi_{9}\left(S^{5}\right) \stackrel{p^{*}}{\longrightarrow}\left[\Sigma^{3} K, S^{5}\right]_{0} \stackrel{j^{*}}{\longrightarrow} \pi_{8}\left(S^{5}\right) \stackrel{8}{\longrightarrow} \pi_{8}\left(S^{5}\right),
$$

com o homomorfismo bordo sendo a multiplicação por 8, pela construção. Do fato que $\pi_{9}\left(S^{5}\right)=\mathbb{Z} / 2\left[\nu_{5} \circ \eta_{8}\right], \pi_{8}\left(S^{5}\right)=\mathbb{Z} / 8\left[\nu_{5}\right]$, temos a seqüência exata de grupos

$$
0 \longrightarrow \mathbb{Z} / 2\left[\nu_{5} \circ \eta_{8}\right] \stackrel{p^{*}}{\longrightarrow}\left[\Sigma^{3} K, S^{5}\right]_{0} \stackrel{j^{*}}{\longrightarrow} \mathbb{Z} / 8\left[\nu_{5}\right] \longrightarrow 0 .
$$

Isso significa que existem duas extensões de $\nu_{5}$, ambas não triviais e relacionadas pela co-ação de Hilton, a saber, se denotarmos uma por $\bar{\nu}_{5}$, a outra será $\bar{\nu}_{5}^{\nu_{5} \circ \eta_{8}}$.

Consideremos o diagrama

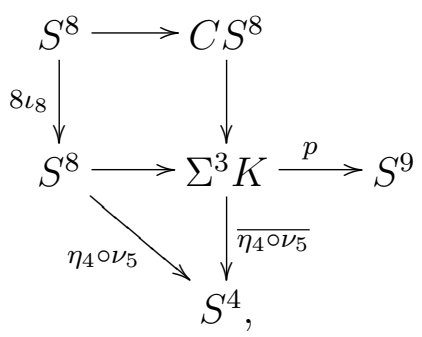

Aplicando novamente a construção de Puppe com $S^{4}$ ao invés de $S^{5}$, obtemos

$$
\pi_{9}\left(S^{4}\right) \stackrel{8}{\longrightarrow} \pi_{9}\left(S^{4}\right) \stackrel{p^{*}}{\longrightarrow}\left[\Sigma^{3} K, S^{4}\right]_{0} \stackrel{j^{*}}{\longrightarrow} \pi_{8}\left(S^{4}\right) \stackrel{8}{\longrightarrow} \pi_{8}\left(S^{4}\right),
$$

com o homomorfismo bordo sendo a multiplicação por 8, pela construção. Como $\pi_{9}\left(S^{4}\right)=$ $\mathbb{Z} / 2\left[\nu_{4} \circ \eta_{7}^{2}, \Sigma \nu^{\prime} \circ \eta_{7}^{2}\right], \pi_{8}\left(S^{4}\right)=\mathbb{Z} / 2\left[\nu_{4} \circ \eta_{7}, \Sigma \nu^{\prime} \circ \eta_{7}=\eta_{4} \circ \nu_{5}\right]$, temos a seqüência exata de grupos

$$
0 \longrightarrow \mathbb{Z} / 2\left[\nu_{4} \circ \eta_{7}^{2}, \Sigma \nu^{\prime} \circ \eta_{7}^{2}\right] \underset{p^{*}}{\longrightarrow}\left[\Sigma^{3} K, S^{4}\right]_{0} \underset{j^{*}}{\longrightarrow} \mathbb{Z} / 2\left[\nu_{4} \circ \eta_{7}, \eta_{4} \circ \nu_{5}\right] \longrightarrow 0
$$

mostrando que $\overline{\eta_{4} \circ \nu_{5}}$ é um dos quatro elementos de $\left[\Sigma^{3} K, S^{4}\right]_{0}$ que são aplicados em $\eta_{4} \circ \nu_{5}$, e logo, não é trivial.

Seja $x=i_{*}\left(\overline{\eta_{4} \circ \nu_{5}}\right)$, obtido de um desses elementos,

$$
x: \Sigma^{3} K \underset{\overline{\eta_{4} \circ \nu_{5}}}{\longrightarrow} S^{4} \underset{i}{\longrightarrow} M
$$

Agora, $k_{*}(x)=k_{*} i_{*}\left(\overline{\eta_{4} \circ \nu_{5}}\right)=0$, já que $k i \sim c_{0}$. Assim, $\pi(x)=1$ e $\lambda(x)$ é uma auto-equivalência de homotopia de $M$. Denote por $\sigma=\lambda(x)=1^{x}$ a auto-equivalência de homotopia de $M$.

Observe que $x$ pode ser trival em princípio, mas seguirá do próximo cálculo que isso não é possível.

Passo 9. Mostremos que

$$
\left\{\eta_{3}, \Sigma \bar{\nu}^{\prime}, \Sigma^{2} \tilde{\nu}_{5}\right\}_{1}=\left\{\mu_{3}, \mu_{3}+\eta_{3} \circ \epsilon_{4}\right\} \subseteq \pi_{12}\left(S^{3} ; 2\right) .
$$


Pela definição,

$$
\left\{\eta_{3}, \Sigma \bar{\nu}^{\prime}, \Sigma^{2} \tilde{\nu}_{5}\right\}_{1} \in \pi_{12}\left(S^{3} ; 2\right) / G
$$

é uma classe lateral do subrgrupo

$$
G=\left[\Sigma^{3} K, S^{3}\right] \circ \Sigma^{3} \tilde{\nu}_{5}+\eta_{3} \circ \pi_{12}\left(S^{3}\right) .
$$

Por [46] (6.4) pág. 56, $\left[\Sigma^{3} K, S^{3}\right] \circ \Sigma^{3} \tilde{\nu}_{5}=0$. Logo

$$
G=\eta_{3} \circ \pi_{12}\left(S^{3}\right) .
$$

Então,

$$
\pi_{12}\left(S^{3} ; 2\right) / G=\mathbb{Z} / 2\left[\mu_{3}, \eta_{3} \circ \epsilon_{4}\right] / \mathbb{Z} / 2\left[\eta_{3} \circ \epsilon_{4}\right] .
$$

Denotemos $x=\mu_{3}, y=\eta_{3} \circ \epsilon_{4}$. Logo, as classes laterais de $\{0, y\}$ em $\{0, x, y, x+y\}$ são

$$
\begin{aligned}
0+\{0, y\} & =\{0, y\}, \\
x+\{0, y\} & =\{x, x+y\}, \\
y+\{0, y\} & =\{y, 0\}, \\
x+y+\{0, y\} & =\{x+y, x\}
\end{aligned}
$$

e assim,

$$
\pi_{12}\left(S^{3} ; 2\right) / G=\mathbb{Z} / 2[x, y] / \mathbb{Z} / 2[y]=\{\{0, y\},\{x, x+y\}\} .
$$

Sabemos que $\mu_{3}=x \in\left\{\eta_{3}, \Sigma \bar{\nu}^{\prime}, \Sigma^{2} \tilde{\nu}_{5}\right\}_{1}$, e segue

$$
\left\{\eta_{3}, \Sigma \bar{\nu}^{\prime}, \Sigma^{2} \tilde{\nu}_{5}\right\}_{1}=\left\{\mu_{3}, \mu_{3}+\eta_{3} \circ \epsilon_{4}\right\} \subseteq \pi_{12}\left(S^{3} ; 2\right),
$$

como desejado.

Passo 10. Recordemos toda a situação descrita no final do Passo 4 (veja também o começo do Passo 8). Temos duas aplicações

$$
f, g: S^{3} \rightarrow L^{12} S^{3}
$$

e precisamos de uma equivalência de homotopia $s$ de $L^{12} S^{3}$ tal que $g \sim s f$. Pela definição,

$$
L^{12} S^{3}=\bigcup_{x \in S^{3}}\left\{h_{x} \in \Omega^{12} S^{3} \mid h_{x}=\nu^{\prime} \circ\left(\iota_{3} \wedge \bar{\eta}_{3}^{M}\right) \circ\left(\iota_{3} \wedge \tilde{\gamma}\right)\left(x \wedge_{-}\right), \Sigma \tilde{\gamma} \in \Omega^{12} M\right\}
$$

e logo, esse é um subespaço do espaço $m_{0}\left(S^{12}, M, S^{3}\right)$. Assim, podemos considerar as duas aplicações

$$
f, g: S^{3} \rightarrow m_{0}\left(S^{12}, M, S^{3}\right)
$$

e precisamos de uma equivalência de homotopia $s$ de $m_{0}\left(S^{12}, M, S^{3}\right)$ tal que $g \sim s f$. Usando o diagrama (como descrito no começo do Passo 8)

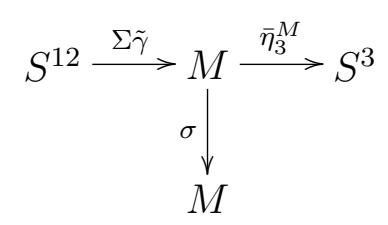


sendo $\sigma$ uma equivalência de homotopia, e a construção feita no Passo 6, a aplicação

$$
\begin{aligned}
& \sigma_{\#}: m_{0}\left(S^{12}, M, S^{3}\right) \rightarrow m_{0}\left(S^{12}, M, S^{3}\right), \\
& \sigma_{\#}: \bar{\eta}_{3}^{M} \circ f \mapsto \bar{\eta}_{3}^{M} \circ \sigma_{*} f
\end{aligned}
$$

é uma equivalência de homotopia. Recordando a forma explícita das aplicações $f$ e $g$ dadas no final do Passo 4,

$$
\begin{aligned}
& f: x \mapsto h_{x}=\nu^{\prime} \circ\left(\iota_{3} \wedge \bar{\eta}_{3}^{M}\right) \circ\left(\iota_{3} \wedge \Sigma \tilde{\gamma}_{1}\right)\left(x \wedge{ }_{-}\right), \\
& g: x \mapsto h_{x}=\nu^{\prime} \circ\left(\iota_{3} \wedge \bar{\eta}_{3}^{M}\right) \circ\left(\iota_{3} \wedge\left(\Sigma \tilde{\gamma}_{1}+\Sigma \gamma_{2}\right)\right)\left(x \wedge_{-}\right)
\end{aligned}
$$

Para mostrar que $\sigma_{\#} f=g$, precisamos mostrar que $\sigma_{*} \Sigma \tilde{\gamma}_{1}=\Sigma \tilde{\gamma}_{1}+\Sigma \gamma_{2}$, com $\tilde{\gamma}_{1}=\widetilde{\Sigma \tilde{\nu}_{5}}$ e $\gamma_{2}=j_{*} \tilde{\nu}_{5} \circ \eta_{10}\left(\sigma_{\#}\left(\mu_{3}\right)=\bar{\eta}_{3}^{M} \circ \sigma_{*}\left(\Sigma \tilde{\gamma}_{1}\right)\right)$.

Primeiro, temos o seguinte resultado:

Lema 4.3.13. Considere o seguinte diagrama:

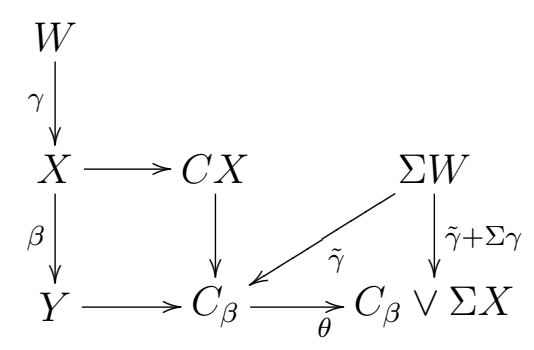

com $\beta \gamma=0, \tilde{\gamma}$ é uma co-extensão de $\gamma$ e $\theta$ é a aplicação de identificação. Então, o triângulo da direita comuta, a saber $\theta \tilde{\gamma}=\tilde{\gamma}+\Sigma \gamma$.

Demonstração: Assuma que $\beta \circ \gamma \sim_{B} 0: W \rightarrow Y$, por alguma homotopia nula $B$ : $C W \rightarrow Y$. Então, uma co-extensão é definida como

$$
\tilde{\gamma}(\langle w, t\rangle)= \begin{cases}\langle\gamma(w), 1-2 t\rangle, & 0 \leq t \leq \frac{1}{2} \\ B(w, 2 t-1), & \frac{1}{2} \leq t \leq 1\end{cases}
$$

Assim, temos o diagrama

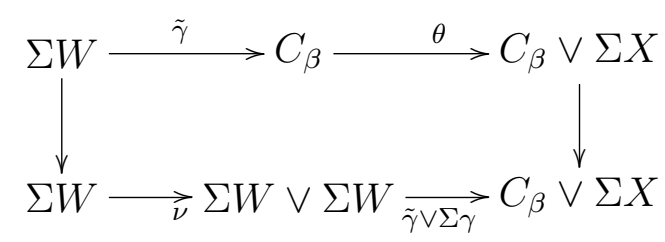

que comuta.

Pelo Lema 4.3.13, e como $\tilde{\gamma}_{1}=\widetilde{\Sigma \tilde{\nu}_{5}}$,

$$
\theta \Sigma \tilde{\gamma}_{1}=\left(\Sigma \tilde{\gamma}_{1} \vee \Sigma^{3} \tilde{\nu}_{5}\right) \nu=\Sigma \tilde{\gamma}_{1}+\Sigma^{3} \tilde{\nu}_{5}
$$


Pela definição (veja o Passo 8), $\sigma_{*}\left(\Sigma \tilde{\gamma}_{1}\right)=\nabla(1 \vee x) \theta \Sigma \tilde{\gamma}_{1}$. Logo,

$$
\sigma_{*}\left(\Sigma \tilde{\gamma}_{1}\right)=\nabla(1 \vee x)\left(\Sigma \tilde{\gamma}_{1} \vee \Sigma^{3} \tilde{\nu}_{5}\right) \nu=\nabla\left(\Sigma \tilde{\gamma}_{1} \vee x \circ \Sigma^{3} \tilde{\nu}_{5}\right) \nu=\Sigma \tilde{\gamma}_{1}+x \circ \Sigma^{3} \tilde{\nu}_{5},
$$

em $\pi_{12}(M)$. Assim, queremos mostrar que

$$
x \circ \Sigma^{3} \tilde{\nu}_{5}=\Sigma \gamma_{2}=j_{*} \Sigma \tilde{\nu}_{6} \circ \eta_{11}
$$

Na verdade, recordando o início do Passo 4, o elemento à direita é a co-extensão que, compondo com a extensão $\bar{\eta}_{3}^{M}$, obtemos

$$
\bar{\eta}_{3}^{M} \circ j_{*} \Sigma \tilde{\nu}_{6} \circ \eta_{11}=\epsilon_{3} \circ \eta_{11}
$$

Logo, o que queremos é mostrar que

$$
\bar{\eta}_{3}^{M} \circ x \circ \Sigma^{3} \tilde{\nu}_{5}=\epsilon_{3} \circ \eta_{11}
$$

Note que $\epsilon_{3} \circ \eta_{11}=\eta_{3} \circ \epsilon_{4}$. Recordando a definição de $x$,

$$
\bar{\eta}_{3}^{M} \circ x \circ \Sigma^{3} \tilde{\nu}_{5}=\bar{\eta}_{3}^{M} \circ i_{*}\left(\overline{\eta_{4} \circ \nu_{5}}\right) \circ \Sigma^{3} \tilde{\nu}_{5}=\eta_{3} \circ \overline{\eta_{4} \circ \nu_{5}} \circ \Sigma^{3} \tilde{\nu}_{5} .
$$

Mostramos que $\overline{\eta_{4} \circ \nu_{5}} \circ \Sigma^{3} \tilde{\nu}_{5}=\epsilon_{4}$.

Pela definição e por [46]:

$$
\Sigma \epsilon_{3}=\Sigma\left\{\eta_{3}, \Sigma \nu^{\prime}, \nu_{7}\right\}_{1} \subseteq-\left\{\eta_{4}, \Sigma^{2} \nu^{\prime}, \nu_{8}\right\}_{2} .
$$

Agora, $\left\{\eta_{4}, \Sigma^{2} \nu^{\prime}, \nu_{8}\right\}_{2}$ é um subconjunto de $\pi_{12}\left(S^{4}\right)$, e mais precisamente

$$
\left\{\eta_{4}, \Sigma^{2} \nu^{\prime}, \nu_{8}\right\}_{2} \subset \pi_{12}\left(S^{4}\right) /\left(\pi_{9}\left(S^{4}\right) \circ \nu_{9}+\eta_{4} \circ \Sigma^{2} \pi_{10}\left(S^{3}\right)\right) .
$$

Mas, $\pi_{10}\left(S^{3}, 2\right)=0$ e $\pi_{9}\left(S^{4}\right)=\mathbb{Z} / 2\left[\nu_{4} \circ \eta_{7}^{2}, \Sigma \nu^{\prime} \circ \eta_{7}^{2}\right]$, e $\eta_{8} \circ \nu_{9}=0$ (por [46] (5.9)). Assim, $\left\{\eta_{4}, \Sigma^{2} \nu^{\prime}, \nu_{8}\right\}_{2}$ é classe lateral do grupo trivial na 2-componente e, logo, é um único elemento da 2-componente.

Então,

$$
\Sigma \epsilon_{3}=\Sigma\left\{\eta_{3}, \Sigma \nu^{\prime}, \nu_{7}\right\}_{1}=-\left\{\eta_{4}, \Sigma^{2} \nu^{\prime}, \nu_{8}\right\}_{2}=-\left\{\eta_{4}, 2 \nu_{5}, \nu_{8}\right\}_{2}
$$

por [46] Proposição 1.3. Agora, por [46] Proposição 1.2 (iii) e (iv),

$$
\left\{\eta_{4}, 2 \nu_{5}, \nu_{8}\right\}_{2} \supseteq\left\{\eta_{4} \circ \nu_{5}, 2 \iota_{8}, \nu_{8}\right\}_{2} \supseteq\left\{\eta_{4} \circ \nu_{5}, 2 \iota_{8}, \nu_{8}\right\}_{3}
$$

e, pela definição, $\left\{\eta_{4} \circ \nu_{5}, 2 \iota_{8}, \nu_{8}\right\}_{3}$ é o conjunto das composições $\overline{\eta_{4} \circ \nu_{5}} \circ \Sigma^{3} \tilde{\nu}_{5}$. Como esse conjunto contém um único elemento, segue que

$$
\overline{\eta_{4} \circ \nu_{5}} \circ \Sigma^{3} \tilde{\nu}_{5}=\epsilon_{4},
$$

como desejado.

Diante de tais resultados, enunciamos a seguinte proposição:

Proposição 4.3.14. Existem quatro $S^{3}$-fibrados principais sobre $S^{13}$ e três tipos diferentes de homotopia dos grupos de gauge desses fibrados.

Demonstração: O resultado segue dos Lemas 4.3.7, 4.3.8, 4.3.9, da Proposição 4.3 .11 e da Observação 4.3.10. 


\subsubsection{O caso de $S^{21}$}

Este caso também merece atenção particular, pois seu comportamento é análogo ao caso de $S^{13}$. Iniciamos com o seguinte resultado:

Lema 4.3.15. Sobre $S^{21}$ existem quarenta e oito $S^{3}$-fibrados principais, classificados pelos elementos $\partial f_{m, n, k, l}$ de $\pi_{20}\left(S^{3}\right)=\mathbb{Z} / 2\left[x=\bar{\mu}_{3}\right] \oplus \mathbb{Z} / 2\left[y=\eta_{3} \circ \mu_{4} \circ \sigma_{13}\right] \oplus \mathbb{Z} / 4\left[z=\bar{\epsilon}^{\prime}\right] \oplus$ $\mathbb{Z} / 3\left[\alpha_{20}\right]$. O tipo de homotopia do grupo de gauge do fibrado trivial é igual ao do fibrado $f_{\bar{\epsilon}^{\prime}}$ e é diferente dos tipos de homotopia dos grupos de gauge dos demais fibrados.

Demonstração: Sabemos que

$$
\begin{gathered}
\partial \beta_{f} \in \pi_{20}\left(S^{3}\right) \approx \mathbb{Z} / 12 \oplus \mathbb{Z} / 2 \oplus \mathbb{Z} / 2, \\
\partial \beta_{f}(2) \in \pi_{20}\left(S^{3} ; 2\right)=\mathbb{Z} / 4\left[\bar{\epsilon}^{\prime}\right] \oplus \mathbb{Z} / 2\left[\bar{\mu}_{3}\right] \oplus \mathbb{Z} / 2\left[\eta_{3} \circ \mu_{4} \circ \sigma_{13}\right] .
\end{gathered}
$$

Então temos 48 fibrados. Pela Proposição 4.1 .9 (com $k=4)$ precisamos considerar apenas

$$
\nu^{\prime} \circ \Sigma^{3}\left(\bar{\epsilon}^{\prime}\right) \stackrel{(21)}{=} \nu^{\prime} \circ \Sigma^{2}\left(\Sigma \nu^{\prime} \circ \kappa_{7}\right) \stackrel{(5)}{=} \nu^{\prime} \circ 2 \nu_{6} \circ \kappa_{9}=0
$$

já que $\nu^{\prime} \circ \nu_{6} \in \pi_{9}^{3}=0, \mathrm{e}$

$$
\nu^{\prime} \circ \Sigma^{3}\left(\bar{\mu}_{3}\right)=\nu^{\prime} \circ \bar{\mu}_{6}
$$

e

$$
\nu^{\prime} \circ \Sigma^{3}\left(\eta_{3} \circ \mu_{4} \circ \sigma_{13}\right)=\nu^{\prime} \circ \eta_{6} \circ \mu_{7} \circ \sigma_{16}
$$

em $\pi_{23}\left(S^{3} ; 2\right)=\mathbb{Z} / 2\left[\nu^{\prime} \circ \bar{\mu}_{6}\right] \oplus \mathbb{Z} / 2\left[\nu^{\prime} \circ \eta_{6} \circ \mu_{7} \circ \sigma_{16}\right]$. Então temos, precisamente, cada um dos geradores e como eles têm ordem 2 , segue $\operatorname{Im} e v_{4}\left(\left[f_{\left[\bar{\mu}_{3}\right]}\right)=\operatorname{Im} e v_{4}\left(\left[f_{\left[\eta_{3} \circ \mu_{4} \circ \sigma_{13}\right]}\right)=2 \mathbb{Z}\right.\right.$ e $\operatorname{Im} e v_{4}\left(\left[f_{\left[\bar{\epsilon}^{\prime}\right]}\right)=\mathbb{Z}\right.$. Com isso, temos

$$
\pi_{2}\left(\mathcal{G}_{\left.f_{\left[\bar{\mu}_{3}\right]}\right)}\right) \approx \pi_{2}\left(\mathcal{G}_{f_{\left[\eta_{3} \circ \mu_{4} \circ \sigma_{13}\right]}}\right) \approx \pi_{23}\left(S^{3}\right) / \mathbb{Z} / 2
$$

e

$$
\pi_{2}\left(\mathcal{G}_{f_{\left[\bar{\epsilon}^{\prime}\right]}}\right) \approx \pi_{2}\left(\mathcal{G}_{c_{0}}\right) \approx \pi_{23}\left(S^{3}\right)
$$

Pelo Lema 4.2.7 temos que $\mathcal{G}_{f_{\left[\bar{\epsilon}^{\prime}\right]}} \sim \mathcal{G}_{c_{0}}$.

Com as informações que temos dos grupos de homotopia de $S^{3}$ conseguimos calcular apenas $\pi_{3}$ e $\pi_{4}$ dos grupos de gauge dos fibrados envolvidos. Esses cálculos nos permitem distingüir mais um tipo diferente de grupo de gauge, como podemos ver na seguinte proposição:

Proposição 4.3.16. Para $n=3,4$, temos $\pi_{n}\left(\mathcal{G}_{f_{(1,1, k, l)}}\right) \approx \pi_{n}\left(\mathcal{G}_{f_{(1,0, k, l)}}\right) \not \approx \pi_{n}\left(\mathcal{G}_{f_{(0,1, k, l)}}\right)$, sendo $\partial f_{m, n, k, l} \in \pi_{20}\left(S^{3}\right)=\mathbb{Z} / 2\left[x=\bar{\mu}_{3}\right] \oplus \mathbb{Z} / 2\left[y=\eta_{3} \circ \mu_{4} \circ \sigma_{13}\right] \oplus \mathbb{Z} / 4\left[z=\bar{\epsilon}^{\prime}\right] \oplus \mathbb{Z} / 3\left[\alpha_{20}\right]$ os elementos que classificam os fibrados sobre $S^{21}$. 
Demonstração: Caso $\pi_{3}\left(\mathcal{G}_{f}\right)$. Consideremos a seqüência no Lema $4.1 .2(\operatorname{com} k=4)$ :

$$
\begin{gathered}
\pi_{4}\left(S^{3}\right) \underset{\partial_{5}}{\longrightarrow} \pi_{24}\left(S^{3}\right) \underset{\sigma_{3}}{\longrightarrow}\left(\mathcal{G}_{f}\right) \underset{e v_{4}}{\longrightarrow} \pi_{3}\left(S^{3}\right) \underset{\partial_{4}}{\longrightarrow} \pi_{23}\left(S^{3}\right), \\
\mathbb{Z} / 2 \underset{\partial_{5}}{\longrightarrow} \mathbb{Z} / 2 \underset{\phi}{\longrightarrow} \pi_{3}\left(\mathcal{G}_{f}\right) \underset{\mathrm{ev}_{4}}{\longrightarrow} \mathbb{Z} \underset{\partial_{4}}{\longrightarrow} \mathbb{Z} / 2 \oplus \mathbb{Z} / 2 .
\end{gathered}
$$

É claro que

$$
\operatorname{Nuc} \phi=\operatorname{Im} \partial_{5}=0 \text { ou } \mathbb{Z} / 2 \text {. }
$$

Assim

$$
\operatorname{Im} \phi=(\mathbb{Z} / 2) / \operatorname{Nuc} \phi=\mathbb{Z} / 2 \text { ou } 0 .
$$

Agora, como um grupo abeliano finitamente gerado, $A=\pi_{3}\left(\mathcal{G}_{f}\right) \approx \mathbb{Z}^{m} \oplus T$, sendo $T$ o subgrupo de torção. Seja $f \neq c_{0}$. Então sabemos pelos cálculos do Lema anterior que

$$
\operatorname{Im} e v_{4}=2 \mathbb{Z}
$$

para o caso $(m, n, k, l) \neq\left(0,0, k^{\prime}, l^{\prime}\right)$, e

$$
\operatorname{Im} e v_{4}=\mathbb{Z}
$$

para o caso $(0,0, k, l)$.

Logo

$$
2 \mathbb{Z}=\operatorname{Im} e v_{4}=A / \mathrm{Nuc} e v_{4}=\left(\mathbb{Z}^{m} \oplus T\right) / \operatorname{Nuc} e v_{4}=\left(\mathbb{Z}^{m} \oplus T\right) / \operatorname{Im} \phi,
$$

implica que $m=1$ e $T=\mathbb{Z} / 2$ ou $T=0$ e

$$
\mathbb{Z}=\operatorname{Im} e v_{4}=A / \operatorname{Nuc} e v_{4}=\left(\mathbb{Z}^{m} \oplus T\right) / \operatorname{Nuc} e v_{4}=\left(\mathbb{Z}^{m} \oplus T\right) / \operatorname{Im} \phi
$$

implica que $m=1$ e $T=\mathbb{Z} / 2$ ou $T=0$.

Para decidir qual é o caso, consideremos que

$$
\operatorname{Im} \phi=(\mathbb{Z} / 2) / \operatorname{Nuc} \phi
$$

$$
\text { Nuc } \phi=\operatorname{Im} \partial_{5}=0 \text { ou } \mathbb{Z} / 2 \text {, }
$$

e

$$
\operatorname{Im} \partial_{5}=(\mathbb{Z} / 2) / \operatorname{Im} e v_{5} .
$$

Precisamos calcular Im $e v_{5}$. Pela Proposição 4.1 .8 (com $\left.k=5\right)$, precisamos resolver a equação

$$
\nu^{\prime} \circ \Sigma^{3} \partial \beta_{f}(2) \circ \Sigma^{20} \partial \omega(2)=0
$$

em $\pi_{24}\left(S^{3} ; 2\right)=\mathbb{Z} / 2\left[\nu^{\prime} \circ \eta_{6} \circ \bar{\mu}_{7}\right]$, com

$$
\partial \beta_{f}(2) \in \pi_{20}\left(S^{3} ; 2\right)=\mathbb{Z} / 4\left[\bar{\epsilon}^{\prime}\right] \oplus \mathbb{Z} / 2\left[\bar{\mu}_{3}\right] \oplus \mathbb{Z} / 2\left[\eta_{3} \circ \mu_{4} \circ \sigma_{13}\right]
$$

e

$$
\partial \omega(2) \in \pi_{4}\left(S^{3} ; 2\right)=\mathbb{Z} / 2\left[\eta_{3}\right]
$$


Então calculemos $\nu^{\prime} \circ \Sigma^{3} \partial \beta_{f}(2)$ para as três possibilidades

$$
\begin{gathered}
\nu^{\prime} \circ \Sigma^{3} \bar{\epsilon}^{\prime}=0, \\
\nu^{\prime} \circ \Sigma^{3}\left(\bar{\mu}_{3}\right)=\nu^{\prime} \circ \bar{\mu}_{6}
\end{gathered}
$$

e

$$
\nu^{\prime} \circ \Sigma^{3}\left(\eta_{3} \circ \mu_{4} \circ \sigma_{13}\right)=\nu^{\prime} \circ \eta_{6} \circ \mu_{7} \circ \sigma_{16},
$$

em $\pi_{23}\left(S^{3} ; 2\right)=\mathbb{Z} / 2\left[\nu^{\prime} \circ \bar{\mu}_{6}\right] \oplus \mathbb{Z} / 2\left[\nu^{\prime} \circ \eta_{6} \circ \mu_{7} \circ \sigma_{16}\right]$.

Compondo com o possível

$$
\Sigma^{20} \partial \omega=(n \bmod 2) \eta_{23},
$$

obtemos elementos de $\pi_{24}\left(S^{3} ; 2\right)$. O primeiro é 0 , o segundo é:

$$
\nu^{\prime} \circ \bar{\mu}_{6} \circ \eta_{23} \stackrel{(1)}{=} \nu^{\prime} \circ \eta_{6} \circ \bar{\mu}_{7},
$$

o qual é o gerador de $\pi_{24}\left(S^{3} ; 2\right)$.

O terceiro é:

$$
\begin{gathered}
\nu^{\prime} \circ \eta_{6} \circ \mu_{7} \circ \sigma_{16} \circ \eta_{23} \stackrel{(1)}{=} \nu^{\prime} \circ \eta_{6} \circ \mu_{7} \circ \eta_{16} \circ \sigma_{17} \stackrel{(1)}{=} \nu^{\prime} \circ \eta_{6} \circ \eta_{7} \circ \mu_{8} \circ \sigma_{17} \\
\stackrel{(12)}{=} \nu^{\prime} \circ \Sigma^{3}\left(2 \mu^{\prime}\right) \circ \sigma_{17} \stackrel{(17)}{=} \nu^{\prime} \circ 2 \Sigma\left(2 \zeta_{5}\right) \circ \sigma_{17}=4 \nu^{\prime} \circ \zeta_{6} \circ \sigma_{17}=0 .
\end{gathered}
$$

Recordemos que denotamos as três aplicações não triviais por $f_{m, n, k, l}, 0 \leq m, n \leq 1$. Logo,

$$
\begin{gathered}
\operatorname{Im} e v_{5}\left(\left[f_{1,0, k, l}\right]\right)=0, \\
\operatorname{Im} e v_{5}\left(\left[f_{0,1, k, l}\right]\right)=\mathbb{Z} / 2, \\
\operatorname{Im} e v_{5}\left(\left[f_{1,1, k, l}\right]\right)=0 . \\
\pi_{3}\left(\mathcal{G}_{f_{(1,1, k, l),(1,0, k, l)}}\right) \approx \mathbb{Z},
\end{gathered}
$$

e

$$
\pi_{3}\left(\mathcal{G}_{f_{(0,1, k, l)}}\right) \approx \mathbb{Z} \oplus \mathbb{Z} / 2
$$

Caso $\pi_{4}\left(\mathcal{G}_{f}\right)$. Consideremos a seqüência no lema $4.1 .2(\operatorname{com} k=5)$ :

$$
\begin{gathered}
\pi_{5}\left(S^{3}\right) \underset{\partial_{6}}{\longrightarrow} \pi_{25}\left(S^{3}\right) \longrightarrow \pi_{4}\left(\mathcal{G}_{f}\right) \underset{e v_{5}}{\longrightarrow} \pi_{4}\left(S^{3}\right) \underset{\partial_{5}}{\longrightarrow} \pi_{24}\left(S^{3}\right), \\
\mathbb{Z} / 2 \underset{\partial_{6}}{\longrightarrow} \mathbb{Z} / 210 \underset{\phi}{\longrightarrow} \pi_{4}\left(\mathcal{G}_{f}\right) \underset{\text { ev }}{\longrightarrow} \mathbb{Z} / 2 \underset{\partial_{5}}{\longrightarrow} \mathbb{Z} / 2 .
\end{gathered}
$$

Temos que

$$
\operatorname{Nuc} \phi=\operatorname{Im} \partial_{6}=0 \text { ou } \mathbb{Z} / 2 \text {. }
$$

Assim

$$
\operatorname{Im} \phi=(\mathbb{Z} / 210) / \mathrm{Nuc} \phi=\mathbb{Z} / 210 \text { ou } \mathbb{Z} / 105 \text {. }
$$


Agora, como um grupo abeliano finitamente gerado, $A=\pi_{4}\left(\mathcal{G}_{f}\right) \approx \mathbb{Z}^{m} \oplus T$, sendo $T$ o subgrupo de torção. Seja $f \neq c_{0}$. Então sabemos pelos cálculos acima que

$$
\operatorname{Im} e v_{4}=0
$$

para os casos $(1,0, k, l)$ e $(1,1, k, l)$, e

$$
\operatorname{Im} e v_{4}=\mathbb{Z} / 2
$$

para o caso $(0,1, k, l)$.

Logo,

$$
0=\operatorname{Im} e v_{5}=A / \operatorname{Nuc} e v_{5}=\left(\mathbb{Z}^{m} \oplus T\right) / \operatorname{Nuc} e v_{5}=\left(\mathbb{Z}^{m} \oplus T\right) / \operatorname{Im} \phi
$$

implica que $m=0$ e $T=\mathbb{Z} / 210$ ou $T=\mathbb{Z} / 105$, e

$$
\mathbb{Z} / 2=\operatorname{Im} e v_{5}=A / \operatorname{Nuc} e v_{5}=\left(\mathbb{Z}^{m} \oplus T\right) / \operatorname{Nuc} e v_{5}=\left(\mathbb{Z}^{m} \oplus T\right) / \operatorname{Im} \phi
$$

implica que $m=0$ e $T=\mathbb{Z} / 2 \oplus \mathbb{Z} / 210$ ou $T=\mathbb{Z} / 210$.

Para decidir qual é o caso, consideremos que

$$
\operatorname{Im} \phi=(\mathbb{Z} / 210) / \text { Nuc } \phi
$$

$$
\operatorname{Nuc} \phi=\operatorname{Im} \partial_{6}=0 \text { ou } \mathbb{Z} / 2 \text {, }
$$

e

$$
\operatorname{Im} \partial_{6}=(\mathbb{Z} / 2) / \operatorname{Im} e v_{6} .
$$

Precisamos calcular Im $e v_{6}$. Pela Proposição $4.1 .8(\operatorname{com} k=6)$, precisamos resolver a equação

$$
\nu^{\prime} \circ \Sigma^{3} \partial \beta_{f}(2) \circ \Sigma^{20} \partial \omega(2)=0
$$

em $\pi_{25}\left(S^{3} ; 2\right)=\mathbb{Z} / 2\left[\epsilon_{3} \circ \kappa_{11}\right]$, com

$$
\partial \beta_{f}(2) \in \pi_{20}\left(S^{3} ; 2\right)=\mathbb{Z} / 4\left[\bar{\epsilon}^{\prime}\right] \oplus \mathbb{Z} / 2\left[\bar{\mu}_{3}\right] \oplus \mathbb{Z} / 2\left[\eta_{3} \circ \mu_{4} \circ \sigma_{13}\right],
$$

e

$$
\partial \omega(2) \in \pi_{5}\left(S^{3} ; 2\right)=\mathbb{Z} / 2\left[\eta_{3}^{2}\right] .
$$

Então calculemos $\nu^{\prime} \circ \Sigma^{3} \partial \beta_{f}(2)$ para as três possibilidades

$$
\begin{gathered}
\nu^{\prime} \circ \Sigma^{3} \bar{\epsilon}^{\prime}=0, \\
\nu^{\prime} \circ \Sigma^{3}\left(\bar{\mu}_{3}\right)=\nu^{\prime} \circ \bar{\mu}_{6}
\end{gathered}
$$

e

$$
\nu^{\prime} \circ \Sigma^{3}\left(\eta_{3} \circ \mu_{4} \circ \sigma_{13}\right)=\nu^{\prime} \circ \eta_{6} \circ \mu_{7} \circ \sigma_{16},
$$

em $\pi_{23}\left(S^{3} ; 2\right)=\mathbb{Z} / 2\left[\nu^{\prime} \circ \bar{\mu}_{6}\right] \oplus \mathbb{Z} / 2\left[\nu^{\prime} \circ \eta_{6} \circ \mu_{7} \circ \sigma_{16}\right]$. 
Compondo com o possível

$$
\Sigma^{20} \partial \omega=(n \bmod 2) \eta_{23}^{2}
$$

obtemos elementos de $\pi_{25}\left(S^{3} ; 2\right)$. O primeiro é 0 , o segundo é:

$$
\begin{gathered}
\nu^{\prime} \circ \bar{\mu}_{6} \circ \eta_{23}^{2} \stackrel{(1)}{=} \nu^{\prime} \circ \eta_{6} \circ \bar{\mu}_{7} \circ \eta_{24} \stackrel{(1)}{=} \nu^{\prime} \circ \eta_{6} \circ \eta_{7} \circ \bar{\mu}_{8} \\
\stackrel{(22)}{=} \nu^{\prime} \circ \Sigma^{3}\left(2 \bar{\mu}^{\prime}\right) \stackrel{(22)}{=} \nu^{\prime} \circ 2 \Sigma\left(2 \bar{\zeta}_{5}\right)=4 \nu^{\prime} \circ \bar{\zeta}_{6}=0 .
\end{gathered}
$$

O terceiro é:

$$
\begin{gathered}
\nu^{\prime} \circ \eta_{6} \circ \mu_{7} \circ \sigma_{16} \circ \eta_{23}^{2} \stackrel{(1)}{=} \nu^{\prime} \circ \eta_{6} \circ \mu_{7} \circ \eta_{16} \circ \sigma_{17} \circ \eta_{24} \stackrel{(1)}{=} \nu^{\prime} \circ \eta_{6} \circ \eta_{7} \circ \mu_{8} \circ \sigma_{17} \circ \eta_{24} \\
\stackrel{(12)}{=} \nu^{\prime} \circ \Sigma^{3}\left(2 \mu^{\prime}\right) \circ \sigma_{17} \circ \eta_{24} \stackrel{(17)}{=} \nu^{\prime} \circ 2 \Sigma\left(2 \zeta_{5}\right) \circ \sigma_{17} \circ \eta_{24}=4 \nu^{\prime} \circ \zeta_{6} \circ \sigma_{17} \circ \eta_{24}=0 .
\end{gathered}
$$

Recordemos que denotamos as três aplicações não triviais por $f_{m, n, k, l}, 0 \leq m, n \leq 1$. Logo,

$$
\begin{gathered}
\operatorname{Im} e v_{6}\left(\left[f_{m, n, k, l}\right]\right)=\mathbb{Z} / 2 . \\
\pi_{4}\left(\mathcal{G}_{f_{(1,1, k, l),(1,0, k, l)}}\right) \approx \mathbb{Z} / 210,
\end{gathered}
$$

e

$$
\pi_{4}\left(\mathcal{G}_{f_{(0,1, k, l)}}\right) \approx \mathbb{Z} / 2 \oplus \mathbb{Z} / 210
$$

Observação 4.3.17. Podemos ver, pela prova do Lema e dos cálculos feitos acima, que

$$
\nu^{\prime} \circ \Sigma^{3}\left(\eta_{3} \circ \mu_{4} \circ \sigma_{13}\right) \circ \Sigma^{20} \partial \omega(2)=0
$$

para qualquer um dos $\partial \omega(2)$ usados (notação da Proposição 4.1.8). Continuando os cálculos, agora só para essa composição, esperamos encontrar pelo menos um caso no qual ela seja diferente de zero. Caso isso ocorra, poderemos ter então $\pi_{n}\left(G_{f_{(1,0, k, l)}}\right) \not$ $\pi_{n}\left(G_{f_{(1,1, k, l)}}\right)$, para algum $n$.

Usando a Proposição 4.1.8 em cada uma dessas composições, consideremos cada caso: Caso $k=6$. Temos

$$
\nu^{\prime} \circ \Sigma^{3} \partial \beta_{f}(2) \circ \Sigma^{20} \partial \omega(2)=0 .
$$

Aqui, $\partial \omega(2)$ está em $\pi_{6}\left(S^{3} ; 2\right)=\mathbb{Z} / 4\left[\nu^{\prime}\right]$.

Compondo

$$
\begin{gathered}
\nu^{\prime} \circ \Sigma^{3}\left(\eta_{3} \circ \mu_{4} \circ \sigma_{13}\right) \circ \Sigma^{20}\left(\nu^{\prime}\right)=\nu^{\prime} \circ \eta_{6} \circ \mu_{7} \circ \sigma_{16} \circ 2 \nu_{23} \\
=\nu^{\prime} \circ \eta_{6} \circ 2 \mu_{7} \circ \sigma_{16} \circ \nu_{23}=0 .
\end{gathered}
$$


Caso $k=7$. Temos

$$
\nu^{\prime} \circ \Sigma^{3} \partial \beta_{f}(2) \circ \Sigma^{20} \partial \omega(2)=0 .
$$

Aqui, $\partial \omega(2)$ está em $\pi_{7}\left(S^{3} ; 2\right)=\mathbb{Z} / 4\left[\nu^{\prime} \circ \eta_{6}\right]$.

Compondo

$$
\begin{gathered}
\nu^{\prime} \circ \Sigma^{3}\left(\eta_{3} \circ \mu_{4} \circ \sigma_{13}\right) \circ \Sigma^{20}\left(\nu^{\prime} \circ \eta_{6}\right)=\nu^{\prime} \circ \eta_{6} \circ \mu_{7} \circ \sigma_{16} \circ 2 \nu_{23} \circ \eta_{26} \\
=\nu^{\prime} \circ \eta_{6} \circ 2 \mu_{7} \circ \sigma_{16} \circ \nu_{23} \circ \eta_{26}=0 .
\end{gathered}
$$

Caso $k=8$. Temos

$$
\nu^{\prime} \circ \Sigma^{3} \partial \beta_{f}(2) \circ \Sigma^{20} \partial \omega(2)=0 .
$$

Aqui, $\partial \omega(2)$ está em $\pi_{8}\left(S^{3} ; 2\right)=\mathbb{Z} / 4\left[\nu^{\prime} \circ \eta_{6}^{2}\right]$.

Compondo

$$
\begin{gathered}
\nu^{\prime} \circ \Sigma^{3}\left(\eta_{3} \circ \mu_{4} \circ \sigma_{13}\right) \circ \Sigma^{20}\left(\nu^{\prime} \circ \eta_{6}^{2}\right)=\nu^{\prime} \circ \eta_{6} \circ \mu_{7} \circ \sigma_{16} \circ 2 \nu_{23} \circ \eta_{26}^{2} \\
=\nu^{\prime} \circ \eta_{6} \circ 2 \mu_{7} \circ \sigma_{16} \circ \nu_{23} \circ \eta_{26}^{2}=0 .
\end{gathered}
$$

Caso $k=9$. Temos

$$
\nu^{\prime} \circ \Sigma^{3} \partial \beta_{f}(2) \circ \Sigma^{20} \partial \omega(2)=0 .
$$

Aqui, $\partial \omega(2)$ está em $\pi_{9}\left(S^{3} ; 2\right)=0$.

Caso $k=10$. Temos

$$
\nu^{\prime} \circ \Sigma^{3} \partial \beta_{f}(2) \circ \Sigma^{20} \partial \omega(2)=0 .
$$

Aqui, $\partial \omega(2)$ está em $\pi_{10}\left(S^{3} ; 2\right)=0$.

Caso $k=11$. Temos

$$
\nu^{\prime} \circ \Sigma^{3} \partial \beta_{f}(2) \circ \Sigma^{20} \partial \omega(2)=0 .
$$

Aqui, $\partial \omega(2)$ está em $\pi_{11}\left(S^{3} ; 2\right)=\mathbb{Z} / 2\left[\epsilon_{3}\right]$.

Compondo

$$
\nu^{\prime} \circ \Sigma^{3}\left(\eta_{3} \circ \mu_{4} \circ \sigma_{13}\right) \circ \Sigma^{20}\left(\epsilon_{3}\right)=\nu^{\prime} \circ \eta_{6} \circ \mu_{7} \circ \sigma_{16} \circ \epsilon_{23} \stackrel{(19)}{=} 0 .
$$

Caso $k=12$. Temos

$$
\nu^{\prime} \circ \Sigma^{3} \partial \beta_{f}(2) \circ \Sigma^{20} \partial \omega(2)=0 .
$$

Aqui, $\partial \omega(2)$ está em $\pi_{12}\left(S^{3} ; 2\right)=\mathbb{Z} / 2\left[\mu_{3}\right] \oplus \mathbb{Z} / 2\left[\eta_{3} \circ \epsilon_{4}\right]$.

Compondo

$$
\nu^{\prime} \circ \Sigma^{3}\left(\eta_{3} \circ \mu_{4} \circ \sigma_{13}\right) \circ \Sigma^{20}\left(\mu_{3}\right)=\nu^{\prime} \circ \eta_{6} \circ \mu_{7} \circ \sigma_{16} \circ \mu_{23} \stackrel{(24)}{=} \nu^{\prime} \circ \eta_{6} \circ \mu_{7} \circ \eta_{16} \circ \rho_{17}
$$




$$
\stackrel{(1)}{=} \nu^{\prime} \circ \eta_{6} \circ \eta_{7} \circ \mu_{8} \circ \rho_{17} \stackrel{(17)}{=} \nu^{\prime} \circ 4 \zeta_{6} \circ \rho_{17}=4 \nu^{\prime} \circ \zeta_{6} \circ \rho_{17}=0
$$

e

$$
\begin{gathered}
\nu^{\prime} \circ \Sigma^{3}\left(\eta_{3} \circ \mu_{4} \circ \sigma_{13}\right) \circ \Sigma^{20}\left(\eta_{3} \circ \epsilon_{4}\right) \stackrel{(1)}{=} \nu^{\prime} \circ \eta_{6} \circ \mu_{7} \circ \sigma_{16} \circ \epsilon_{23} \circ \eta_{31} \\
=\nu^{\prime} \circ \eta_{6} \circ \mu_{7} \circ \sigma_{16} \circ \epsilon_{23} \circ \eta_{31} \stackrel{(19)}{=} 0 .
\end{gathered}
$$

Caso $k=13$. Temos

$$
\nu^{\prime} \circ \Sigma^{3} \partial \beta_{f}(2) \circ \Sigma^{20} \partial \omega(2)=0 .
$$

Aqui, $\partial \omega(2)$ está em $\pi_{13}\left(S^{3} ; 2\right)=\mathbb{Z} / 4\left[\epsilon^{\prime}\right] \oplus \mathbb{Z} / 2\left[\eta_{3} \circ \mu_{4}\right]$.

Compondo

$$
\begin{gathered}
\nu^{\prime} \circ \Sigma^{3}\left(\eta_{3} \circ \mu_{4} \circ \sigma_{13}\right) \circ \Sigma^{20}\left(\epsilon^{\prime}\right)=\nu^{\prime} \circ \eta_{6} \circ \mu_{7} \circ \sigma_{16} \circ 2 \Sigma^{18}\left(\nu_{5} \circ \sigma_{8}\right) \\
=\nu^{\prime} \circ \eta_{6} \circ 2 \mu_{7} \circ \sigma_{16} \circ \Sigma^{18}\left(\nu_{5} \circ \sigma_{8}\right)=0
\end{gathered}
$$

e

$$
\begin{gathered}
\nu^{\prime} \circ \Sigma^{3}\left(\eta_{3} \circ \mu_{4} \circ \sigma_{13}\right) \circ \Sigma^{20}\left(\eta_{3} \circ \mu_{4}\right) \stackrel{(1)}{=} \nu^{\prime} \circ \mu_{6} \circ \sigma_{15} \circ \eta_{22} \circ \eta_{23} \circ \mu_{24} \\
\stackrel{(12)}{=} \nu^{\prime} \circ \mu_{6} \circ \sigma_{15} \circ \Sigma^{19}\left(2 \mu^{\prime}\right)=\nu^{\prime} \circ 2 \mu_{6} \circ \sigma_{15} \circ \Sigma^{19} \mu^{\prime}=0 .
\end{gathered}
$$

Caso $k=14$. Temos

$$
\nu^{\prime} \circ \Sigma^{3} \partial \beta_{f}(2) \circ \Sigma^{20} \partial \omega(2)=0 .
$$

Aqui, $\partial \omega(2)$ está em $\pi_{14}\left(S^{3} ; 2\right)=\mathbb{Z} / 4\left[\mu^{\prime}\right] \oplus \mathbb{Z} / 2\left[\epsilon_{3} \circ \nu_{4}\right] \oplus \mathbb{Z} / 2\left[\nu^{\prime} \circ \epsilon_{6}\right]$.

Compondo

$$
\begin{gathered}
\nu^{\prime} \circ \Sigma^{3}\left(\eta_{3} \circ \mu_{4} \circ \sigma_{13}\right) \circ \Sigma^{20}\left(\mu^{\prime}\right) \stackrel{(17)}{=} \nu^{\prime} \circ \eta_{6} \circ \mu_{7} \circ \sigma_{16} \circ \Sigma^{18}\left(2 \zeta_{5}\right)=\nu^{\prime} \circ \eta_{6} \circ 2 \mu_{7} \circ \sigma_{16} \circ \Sigma^{18} \zeta_{5}=0, \\
\nu^{\prime} \circ \Sigma^{3}\left(\eta_{3} \circ \mu_{4} \circ \sigma_{13}\right) \circ \Sigma^{20}\left(\epsilon_{3} \circ \nu_{4}\right)=\nu^{\prime} \circ \eta_{6} \circ \mu_{7} \circ \sigma_{16} \circ \epsilon_{23} \circ \nu_{24} \stackrel{(19)}{=} 0
\end{gathered}
$$

e

$$
\nu^{\prime} \circ \Sigma^{3}\left(\eta_{3} \circ \mu_{4} \circ \sigma_{13}\right) \circ \Sigma^{20}\left(\nu^{\prime} \circ \epsilon_{6}\right)=\nu^{\prime} \circ \eta_{6} \circ \mu_{7} \circ \sigma_{16} \circ 2 \nu_{23} \circ \epsilon_{26} \stackrel{(5)}{=} 0
$$

Caso $k=15$. Temos

$$
\nu^{\prime} \circ \Sigma^{3} \partial \beta_{f}(2) \circ \Sigma^{20} \partial \omega(2)=0 .
$$

Aqui, $\partial \omega(2)$ está em $\pi_{15}\left(S^{3} ; 2\right)=\mathbb{Z} / 2\left[\nu^{\prime} \circ \mu_{6}\right] \oplus \mathbb{Z} / 2\left[\nu^{\prime} \circ \eta_{6} \circ \epsilon_{7}\right]$.

Compondo

$$
\nu^{\prime} \circ \Sigma^{3}\left(\eta_{3} \circ \mu_{4} \circ \sigma_{13}\right) \circ \Sigma^{20}\left(\nu^{\prime} \circ \mu_{6}\right)=\nu^{\prime} \circ \eta_{6} \circ \mu_{7} \circ \sigma_{16} \circ 2 \nu_{23} \circ \mu_{26} \stackrel{(5)}{=} 0
$$

e

$$
\nu^{\prime} \circ \Sigma^{3}\left(\eta_{3} \circ \mu_{4} \circ \sigma_{13}\right) \circ \Sigma^{20}\left(\nu^{\prime} \circ \eta_{6} \circ \epsilon_{7}\right)=\nu^{\prime} \circ \eta_{6} \circ \mu_{7} \circ \sigma_{16} \circ 2 \nu_{23} \circ \eta_{26} \circ \epsilon_{27} \stackrel{(5)}{=} 0 .
$$


Caso $k=16$. Temos

$$
\nu^{\prime} \circ \Sigma^{3} \partial \beta_{f}(2) \circ \Sigma^{20} \partial \omega(2)=0 .
$$

Aqui, $\partial \omega(2)$ está em $\pi_{16}\left(S^{3} ; 2\right)=\mathbb{Z} / 2\left[\nu^{\prime} \circ \eta_{6} \circ \mu_{7}\right]$.

Compondo

$$
\nu^{\prime} \circ \Sigma^{3}\left(\eta_{3} \circ \mu_{4} \circ \sigma_{13}\right) \circ \Sigma^{20}\left(\nu^{\prime} \circ \eta_{6} \circ \mu_{7}\right)=\nu^{\prime} \circ \eta_{6} \circ \mu_{7} \circ \sigma_{16} \circ 2 \nu_{23} \circ \eta_{26} \circ \mu_{27} \stackrel{(5)}{=} 0 .
$$

Caso $k=17$. Temos

$$
\nu^{\prime} \circ \Sigma^{3} \partial \beta_{f}(2) \circ \Sigma^{20} \partial \omega(2)=0 .
$$

Aqui, $\partial \omega(2)$ está em $\pi_{17}\left(S^{3} ; 2\right)=\mathbb{Z} / 2\left[\epsilon_{3} \circ \nu_{11}^{2}\right]$.

Compondo

$$
\nu^{\prime} \circ \Sigma^{3}\left(\eta_{3} \circ \mu_{4} \circ \sigma_{13}\right) \circ \Sigma^{20}\left(\epsilon_{3} \circ \nu_{11}^{2}\right)=\nu^{\prime} \circ \eta_{6} \circ \mu_{7} \circ \sigma_{16} \circ \epsilon_{23} \circ \nu_{31}^{2} \stackrel{(19)}{=} 0 .
$$

Caso $k=18$. Temos

$$
\nu^{\prime} \circ \Sigma^{3} \partial \beta_{f}(2) \circ \Sigma^{20} \partial \omega(2)=0 .
$$

Aqui, $\partial \omega(2)$ está em $\pi_{18}\left(S^{3} ; 2\right)=\mathbb{Z} / 2\left[\bar{\epsilon}_{3}\right]$.

Compondo

$$
\nu^{\prime} \circ \Sigma^{3}\left(\eta_{3} \circ \mu_{4} \circ \sigma_{13}\right) \circ \Sigma^{20}\left(\bar{\epsilon}_{3}\right) \stackrel{(1)}{=} \nu^{\prime} \circ \mu_{6} \circ \sigma_{15} \circ \eta_{22} \circ \bar{\epsilon}_{23} \stackrel{(23)}{=} \nu^{\prime} \circ \mu_{6} \circ \sigma_{15} \circ \epsilon_{22} \circ \epsilon_{30} \stackrel{(19)}{=} 0 .
$$

Caso $k=19$. Temos

$$
\nu^{\prime} \circ \Sigma^{3} \partial \beta_{f}(2) \circ \Sigma^{20} \partial \omega(2)=0 .
$$

Aqui, $\partial \omega(2)$ está em $\pi_{19}\left(S^{3} ; 2\right)=\mathbb{Z} / 2\left[\mu_{3} \circ \sigma_{12}\right] \oplus \mathbb{Z} / 2\left[\eta_{3} \circ \bar{\epsilon}_{4}\right]$.

Compondo

$$
\begin{gathered}
\nu^{\prime} \circ \Sigma^{3}\left(\eta_{3} \circ \mu_{4} \circ \sigma_{13}\right) \circ \Sigma^{20}\left(\mu_{3} \circ \sigma_{12}\right) \stackrel{(24)}{=} \nu^{\prime} \circ \eta_{6} \circ \mu_{7} \circ \eta_{16} \circ \rho_{17} \circ \sigma_{32} \\
\stackrel{(1)}{=} \nu^{\prime} \circ \eta_{6} \circ \eta_{7} \circ \mu_{8} \circ \rho_{17} \circ \sigma_{32} \stackrel{(17)}{=} \nu^{\prime} \circ 4 \zeta_{6} \circ \rho_{17} \circ \sigma_{32}=0
\end{gathered}
$$

e

$$
\begin{aligned}
& \nu^{\prime} \circ \Sigma^{3}\left(\eta_{3} \circ \mu_{4} \circ \sigma_{13}\right) \circ \Sigma^{20}\left(\eta_{3} \circ \bar{\epsilon}_{4}\right) \stackrel{(23)}{=} \nu^{\prime} \circ \eta_{6} \circ \mu_{7} \circ \sigma_{16} \circ \bar{\epsilon}_{23} \circ \eta_{24} \\
& \stackrel{(1)}{=} \nu^{\prime} \circ \mu_{6} \circ \sigma_{15} \circ \eta_{22} \circ \bar{\epsilon}_{23} \circ \eta_{24} \stackrel{(23)}{=} \nu^{\prime} \circ \mu_{6} \circ \sigma_{15} \circ \epsilon_{22} \circ \epsilon_{30} \circ \eta_{24} \stackrel{(19)}{=} 0 .
\end{aligned}
$$

Caso $k=20$. Temos

$$
\nu^{\prime} \circ \Sigma^{3} \partial \beta_{f}(2) \circ \Sigma^{20} \partial \omega(2)=0 .
$$

Aqui, $\partial \omega(2)$ está em $\pi_{20}\left(S^{3} ; 2\right)=\mathbb{Z} / 4\left[\bar{\epsilon}^{\prime}\right] \oplus \mathbb{Z} / 2\left[\bar{\mu}_{3}\right] \oplus \mathbb{Z} / 2\left[\eta_{3} \circ \mu_{4} \circ \sigma_{13}\right]$. 
Compondo

$$
\begin{gathered}
\nu^{\prime} \circ \Sigma^{3}\left(\eta_{3} \circ \mu_{4} \circ \sigma_{13}\right) \circ \Sigma^{20}\left(\bar{\epsilon}^{\prime}\right) \stackrel{(21)}{=} \nu^{\prime} \circ \eta_{6} \circ \mu_{7} \circ \sigma_{16} \circ \Sigma^{20} \nu^{\prime} \circ \Sigma^{19} \kappa_{7} \\
\stackrel{(5)}{=} \nu^{\prime} \circ \eta_{6} \circ \mu_{7} \circ \sigma_{16} \circ 2 \nu_{23} \circ \kappa_{26}=0, \\
\nu^{\prime} \circ \Sigma^{3}\left(\eta_{3} \circ \mu_{4} \circ \sigma_{13}\right) \circ \Sigma^{20}\left(\bar{\mu}_{3}\right) \stackrel{(1)}{=} \nu^{\prime} \circ \eta_{6} \circ \mu_{7} \circ \bar{\mu}_{16} \circ \sigma_{33} \stackrel{(1)}{=} \nu^{\prime} \circ \eta_{6} \circ \bar{\mu}_{7} \circ \mu_{24} \circ \sigma_{33} \\
\stackrel{(24)}{=} \nu^{\prime} \circ \eta_{6} \circ \bar{\mu}_{7} \circ \eta_{24} \circ \rho_{25} \stackrel{(1)}{=} \nu^{\prime} \circ \eta_{6} \circ \eta_{7} \circ \bar{\mu}_{8} \circ \rho_{25}=\nu^{\prime} \circ \eta_{6}^{2} \circ \bar{\mu}_{8} \circ \rho_{25} \\
=\nu^{\prime} \circ \Sigma^{3}\left(\eta_{3}^{2} \circ \bar{\mu}_{5}\right) \circ \rho_{25} \stackrel{(22)}{=} \nu^{\prime} \circ \Sigma^{3}\left(2 \bar{\mu}^{\prime}\right) \circ \rho_{25}=2 \nu^{\prime} \circ \Sigma\left(\Sigma^{2} \bar{\mu}^{\prime}\right) \circ \rho_{25} \\
\stackrel{(22)}{=} 2 \nu^{\prime} \circ \Sigma\left(2 \bar{\zeta}_{5}\right) \circ \rho_{25}=4 \nu^{\prime} \circ \bar{\zeta}_{6} \circ \rho_{25}=0
\end{gathered}
$$

e

$$
\begin{gathered}
\nu^{\prime} \circ \Sigma^{3}\left(\eta_{3} \circ \mu_{4} \circ \sigma_{13}\right) \circ \Sigma^{20}\left(\eta_{3} \circ \mu_{4} \circ \sigma_{13}\right) \stackrel{(1)}{=} \nu^{\prime} \circ \mu_{6} \circ \sigma_{15} \circ \eta_{22}^{2} \circ \mu_{24} \circ \sigma_{33} \\
\stackrel{(12)}{=} \nu^{\prime} \circ \mu_{6} \circ \sigma_{15} \circ \Sigma^{19}\left(2 \mu^{\prime}\right) \circ \sigma_{33}=0 .
\end{gathered}
$$

Caso $k=21$. Temos

$$
\nu^{\prime} \circ \Sigma^{3} \partial \beta_{f}(2) \circ \Sigma^{20} \partial \omega(2)=0 .
$$

Aqui, $\partial \omega(2)$ está em $\pi_{21}\left(S^{3} ; 2\right)=\mathbb{Z} / 4\left[\mu^{\prime} \circ \sigma_{14}\right] \oplus \mathbb{Z} / 2\left[\nu^{\prime} \circ \bar{\epsilon}_{6}\right] \oplus \mathbb{Z} / 2\left[\eta_{3} \circ \bar{\mu}_{4}\right]$.

Compondo

$$
\begin{gathered}
\nu^{\prime} \circ \Sigma^{3}\left(\eta_{3} \circ \mu_{4} \circ \sigma_{13}\right) \circ \Sigma^{20}\left(\mu^{\prime} \circ \sigma_{14}\right) \stackrel{(17)}{=} \nu^{\prime} \circ \eta_{6} \circ \mu_{7} \circ \sigma_{16} \circ \Sigma^{18}\left(2 \zeta_{5}\right) \circ \sigma_{34}=0, \\
\nu^{\prime} \circ \Sigma^{3}\left(\eta_{3} \circ \mu_{4} \circ \sigma_{13}\right) \circ \Sigma^{20}\left(\nu^{\prime} \circ \bar{\epsilon}_{6}\right) \stackrel{(5)}{=} \nu^{\prime} \circ \eta_{6} \circ \mu_{7} \circ \sigma_{16} \circ 2 \nu_{23} \circ \bar{\epsilon}_{26}=0
\end{gathered}
$$

e

$$
\begin{gathered}
\nu^{\prime} \circ \Sigma^{3}\left(\eta_{3} \circ \mu_{4} \circ \sigma_{13}\right) \circ \Sigma^{20}\left(\eta_{3} \circ \bar{\mu}_{4}\right) \stackrel{(1)}{=} \nu^{\prime} \circ \mu_{6} \circ \sigma_{15} \circ \eta_{22} \circ \eta_{23} \circ \bar{\mu}_{24} \\
\stackrel{(22)}{=} \nu^{\prime} \circ \mu_{6} \circ \sigma_{15} \circ \Sigma^{19}\left(2 \bar{\mu}^{\prime}\right)=0 .
\end{gathered}
$$

Caso $k=22$. Temos

$$
\nu^{\prime} \circ \Sigma^{3} \partial \beta_{f}(2) \circ \Sigma^{20} \partial \omega(2)=0 .
$$

Aqui, $\partial \omega(2)$ está em $\pi_{22}\left(S^{3} ; 2\right)=\mathbb{Z} / 4\left[\bar{\mu}^{\prime}\right] \oplus \mathbb{Z} / 2\left[\nu^{\prime} \circ \mu_{6} \circ \sigma_{15}\right]$.

Compondo

$$
\nu^{\prime} \circ \Sigma^{3}\left(\eta_{3} \circ \mu_{4} \circ \sigma_{13}\right) \circ \Sigma^{20}\left(\bar{\mu}^{\prime}\right) \stackrel{(22)}{=} \nu^{\prime} \circ \eta_{6} \circ \mu_{7} \circ \sigma_{16} \circ \Sigma^{18}\left(2 \bar{\zeta}_{5}\right)=0
$$

e

$$
\nu^{\prime} \circ \Sigma^{3}\left(\eta_{3} \circ \mu_{4} \circ \sigma_{13}\right) \circ \Sigma^{20}\left(\nu^{\prime} \circ \mu_{6} \circ \sigma_{15}\right) \stackrel{(5)}{=} \nu^{\prime} \circ \eta_{6} \circ \mu_{7} \circ \sigma_{16} \circ 2 \nu_{23} \circ \mu_{26} \circ \sigma_{35}=0
$$

Caso $k=23$. Temos

$$
\nu^{\prime} \circ \Sigma^{3} \partial \beta_{f}(2) \circ \Sigma^{20} \partial \omega(2)=0
$$


Aqui, $\partial \omega(2)$ está em $\pi_{23}\left(S^{3} ; 2\right)=\mathbb{Z} / 2\left[\nu^{\prime} \circ \bar{\mu}_{6}\right] \oplus \mathbb{Z} / 2\left[\nu^{\prime} \circ \eta_{6} \circ \mu_{7} \circ \sigma_{16}\right]$.

Compondo

$$
\nu^{\prime} \circ \Sigma^{3}\left(\eta_{3} \circ \mu_{4} \circ \sigma_{13}\right) \circ \Sigma^{20}\left(\nu^{\prime} \circ \bar{\mu}_{6}\right) \stackrel{(5)}{=} \nu^{\prime} \circ \eta_{6} \circ \mu_{7} \circ \sigma_{16} \circ 2 \nu_{23} \circ \bar{\mu}_{26}=0
$$

$\mathrm{e}$

$\nu^{\prime} \circ \Sigma^{3}\left(\eta_{3} \circ \mu_{4} \circ \sigma_{13}\right) \circ \Sigma^{20}\left(\nu^{\prime} \circ \eta_{6} \circ \mu_{7} \circ \sigma_{16}\right) \stackrel{(5)}{=} \nu^{\prime} \circ \eta_{6} \circ \mu_{7} \circ \sigma_{16} \circ 2 \nu_{23} \circ \eta_{26} \circ \mu_{27} \circ \sigma_{36}=0$.

Caso $k=24$. Temos

$$
\nu^{\prime} \circ \Sigma^{3} \partial \beta_{f}(2) \circ \Sigma^{20} \partial \omega(2)=0 .
$$

Aqui, $\partial \omega(2)$ está em $\pi_{24}\left(S^{3} ; 2\right)=\mathbb{Z} / 2\left[\nu^{\prime} \circ \eta_{6} \circ \bar{\mu}_{7}\right]$.

Compondo

$$
\nu^{\prime} \circ \Sigma^{3}\left(\eta_{3} \circ \mu_{4} \circ \sigma_{13}\right) \circ \Sigma^{20}\left(\nu^{\prime} \circ \eta_{6} \circ \bar{\mu}_{7}\right) \stackrel{(5)}{=} \nu^{\prime} \circ \eta_{6} \circ \mu_{7} \circ \sigma_{16} \circ 2 \nu_{23} \circ \eta_{26} \circ \bar{\mu}_{27}=0
$$

Caso $k=25$. Temos

$$
\nu^{\prime} \circ \Sigma^{3} \partial \beta_{f}(2) \circ \Sigma^{20} \partial \omega(2)=0
$$

Aqui, $\partial \omega(2)$ está em $\pi_{25}\left(S^{3} ; 2\right)=\mathbb{Z} / 2\left[\epsilon_{3} \circ \kappa_{11}\right]$.

Compondo

$$
\nu^{\prime} \circ \Sigma^{3}\left(\eta_{3} \circ \mu_{4} \circ \sigma_{13}\right) \circ \Sigma^{20}\left(\epsilon_{3} \circ \kappa_{11}\right)=\nu^{\prime} \circ \eta_{6} \circ \mu_{7} \circ \sigma_{16} \circ \epsilon_{23} \circ \kappa_{31} \stackrel{(19)}{=} 0 .
$$

Infelizmente, não encontramos mais informações na literatura sobre os geradores dos grupos envolvidos. Sendo assim, formulamos a seguinte conjectura quanto aos tipos de homotopia dos grupos de gauge dos $S^{3}$-fibrados principais sobre $S^{21}$ :

Conjectura 4.3.18. Sobre $S^{21}$ existem quarenta e oito $S^{3}$-fibrados principais, classificados pelos elementos $\partial f_{m, n, k, l}$ de $\pi_{20}\left(S^{3}\right)=\mathbb{Z} / 2\left[x=\bar{\mu}_{3}\right] \oplus \mathbb{Z} / 2\left[y=\eta_{3} \circ \mu_{4} \circ \sigma_{13}\right] \oplus \mathbb{Z} / 4[z=$ $\left.\bar{\epsilon}^{\prime}\right] \oplus \mathbb{Z} / 3\left[\alpha_{20}\right]$. Os grupos de gauge são de três tipos de homotopia: um de $f_{0,0, l, k}$, outro de $f_{1, n, l, k}$, e mais outro de $f_{0,1, l, k}(m, n, l, k \in \mathbb{Z})$.

Finalizamos aqui, reunindo todos esses resultados na seguinte proposição:

Proposição 4.3.19. A seguinte tabela reúne o número dos $S^{3}$-fibrados principais sobre a esfera $S^{n}$, o número máximo de grupos de gauge (não homotópicos) desses fibrados, e o número de diferentes $\pi_{2}$ desses grupos de gauge (= número de diferentes tipos de homotopia): 


\begin{tabular}{|c|c|c|c|c|c|}
\hline$n$ & $\pi_{n-1}\left(S^{3}\right)$ & $=$ fibrados, $\xi_{\mathrm{f}}$ & $\pi_{n+2}\left(S^{3}\right)$ & $=$ grupos de gauge, $\mathcal{G}_{\mathrm{f}}$ & $\pi_{2}\left(\mathcal{G}_{f}\right)$ \\
\hline 4 & $\mathbb{Z}$ & $\mathbb{Z}$ & $\mathbb{Z} / 12$ & 12 & 6 \\
\hline 5 & $\mathbb{Z} / 2$ & 2 & $\mathbb{Z} / 2$ & 2 & 2 \\
\hline 6 & $\mathbb{Z} / 2$ & 2 & $\mathbb{Z} / 2$ & 2 & 2 \\
\hline 7 & $\mathbb{Z} / 12$ & 12 & $\mathbb{Z} / 3$ & 3 & 1 \\
\hline 8 & $\mathbb{Z} / 2$ & 2 & $\mathbb{Z} / 15$ & 15 & 1 \\
\hline 9 & $\mathbb{Z} / 2$ & 2 & $\mathbb{Z} / 2$ & 2 & 1 \\
\hline 10 & $\mathbb{Z} / 3$ & 3 & $\mathbb{Z} / 2 \oplus \mathbb{Z} / 2$ & 4 & 1 \\
\hline 11 & $\mathbb{Z} / 15$ & 15 & $\mathbb{Z} / 2 \oplus \mathbb{Z} / 12$ & 24 & 1 \\
\hline 12 & $\mathbb{Z} / 2$ & 2 & $(\mathbb{Z} / 2)^{2} \oplus \mathbb{Z} / 84$ & 336 & 2 \\
\hline 13 & $\mathbb{Z} / 2 \oplus \mathbb{Z} / 2$ & 4 & $\mathbb{Z} / 2 \oplus \mathbb{Z} / 2$ & 4 & 2 \\
\hline 14 & $\mathbb{Z} / 2 \oplus \mathbb{Z} / 12$ & 24 & $\mathbb{Z} / 6$ & 6 & 2 \\
\hline 15 & $(\mathbb{Z} / 2)^{2} \oplus \mathbb{Z} / 84$ & 336 & $\mathbb{Z} / 30$ & 30 & 1 \\
\hline 16 & $\mathbb{Z} / 2 \oplus \mathbb{Z} / 2$ & 4 & $\mathbb{Z} / 30$ & 30 & 1 \\
\hline 17 & $\mathbb{Z} / 6$ & 6 & $\mathbb{Z} / 2 \oplus \mathbb{Z} / 6$ & 12 & 1 \\
\hline 18 & $\mathbb{Z} / 30$ & 30 & $(\mathbb{Z} / 2)^{2} \oplus \mathbb{Z} / 12$ & 48 & 1 \\
\hline 19 & $\mathbb{Z} / 30$ & 30 & $(\mathbb{Z} / 2)^{2} \oplus \mathbb{Z} / 12$ & 48 & 2 \\
\hline 20 & $\mathbb{Z} / 2 \oplus \mathbb{Z} / 6$ & 12 & $\mathbb{Z} / 2 \oplus \mathbb{Z} / 132$ & 264 & 2 \\
\hline 21 & $(\mathbb{Z} / 2)^{2} \oplus \mathbb{Z} / 12$ & 48 & $\mathbb{Z} / 2 \oplus \mathbb{Z} / 2$ & 4 & 2 \\
\hline 22 & $(\mathbb{Z} / 2)^{2} \oplus \mathbb{Z} / 12$ & 48 & $\mathbb{Z} / 2$ & 2 & 2 \\
\hline 23 & $\mathbb{Z} / 2 \oplus \mathbb{Z} / 132$ & 264 & $\mathbb{Z} / 210$ & 210 & 1 \\
\hline 24 & $\mathbb{Z} / 2 \oplus \mathbb{Z} / 2$ & 4 & $\pi_{26}^{3} \approx \mathbb{Z} / 4$ & $4 x$ & 1 \\
\hline 25 & $\mathbb{Z} / 2$ & 2 & $\pi_{27}^{3} \approx(\mathbb{Z} / 2)^{3}$ & $8 x$ & 1 \\
\hline
\end{tabular}

E nos casos de $S^{13}$ e $S^{21}$ temos três tipos diferentes para os grupos de gauge.

Demonstração: O resultado segue da Tabela 1, apresentada no início deste capítulo, de [25] quando $n=4$, das Proposições 4.3.1, 4.3.2, 4.3.3, 4.3.4, 4.3.5, 4.3.6, 4.3.14 e da Conjectura 4.3.18. 


\subsection{Apêndice do capítulo 4}

Apresentamos aqui algumas relações úteis para o nosso trabalho que podem ser encontradas em Toda [46].

1. pág. 25 (Prop. 3.1) Seja $\alpha \in \pi_{p+k}\left(S^{p}\right), \beta \in \pi_{q+h}\left(S^{q}\right)$, então $\Sigma^{q} \alpha \circ \Sigma^{p+k} \beta=$ $\pm \Sigma^{p} \beta \circ \Sigma^{q+h} \alpha$.

2. pág. 39 (5.2) A composição com $\eta_{2}: \quad \alpha \rightarrow \eta_{2} \circ \alpha$ define um isomorfismo $\pi_{i}^{3} \approx \pi_{i}^{2}$ para $i \geq 3$.

3. pág. $40(5.3) \nu^{\prime} \in \pi_{6}^{3}, H\left(\nu^{\prime}\right)=\eta_{5}$ e $2 \nu^{\prime}=\eta_{3} \circ \eta_{4} \circ \eta_{5}$.

4. pág. 41 (Lema 5.4) $\nu_{4} \in \pi_{7}^{4}, H\left(\nu_{4}\right)=\iota_{7}$ e $2 \Sigma \nu_{4}=\Sigma^{2} \nu^{\prime}$.

5. pág. $42(5.5) 2 \nu_{n}=\Sigma^{n-3} \nu^{\prime}, 4 \nu_{n}=\eta_{n}^{3}$ e $4 \nu=\eta^{3}$, para $n \geq 5$.

6. pág. $43(5.7) H\left(\nu^{\prime} \circ \eta_{6}\right)=\eta_{5}^{2}$.

7. pág. $43(5.8) P\left(\iota_{9}\right)= \pm\left(2 \nu_{4}-\Sigma \nu^{\prime}\right)= \pm\left[\iota_{4}, \iota_{4}\right]$.

8. pág. 43 (Lema 5.7) Se $\Sigma^{2} \alpha \in 2 \iota_{5} \circ \pi_{i+2}\left(S^{5}\right)$ para um elemento $\alpha \in \pi_{i}\left(S^{3}\right)$, então $\Sigma\left(\eta_{2} \circ \alpha\right)=0$. Em particular $\Sigma\left(\eta_{2} \circ \nu^{\prime}\right)=0$ e $P\left(\nu_{5}\right)= \pm\left(\eta_{2} \circ \nu^{\prime}\right)$.

9. pág. $44(5.9) \eta_{3} \circ \nu_{4}=\nu^{\prime} \circ \eta_{6}, \eta_{n} \circ \nu_{n+1}=0$, para $n \geq 5$ e $\nu_{n} \circ \eta_{n+3}=0$, para $n \geq 6$.

10. pág. 56 (Lema 6.5$) H\left(\mu_{3}\right)=\sigma^{\prime \prime \prime}, 2 \mu_{n}=2 \mu=0$, para $n \geq 3$.

11. pág. $64(7.5) \epsilon_{n} \circ \eta_{n+8}=\eta_{n} \circ \epsilon_{n+1}, n \geq 3$.

12. pág. $66(7.7) H\left(\mu^{\prime}\right)=\mu_{5}$ e $2 \mu^{\prime}=\eta_{3}^{2} \circ \mu_{5}$.

13. pág. $66(7.8) H\left(\nu^{\prime} \circ \epsilon_{6}\right)=H\left(\nu^{\prime}\right) \circ \epsilon_{6}=\eta_{5} \circ \epsilon_{6}$ e $H\left(\epsilon_{3} \circ \nu_{11}\right)=H\left(\epsilon_{3}\right) \circ \nu_{11}=\nu_{5}^{2} \circ \nu_{11}=\nu_{5}^{3}$.

14. pág. $67(7.10) 2\left(\nu_{5} \circ \sigma_{8}\right)= \pm \Sigma^{2} \epsilon^{\prime}$ e $\eta_{n}^{2} \circ \epsilon_{n+2}=4\left(\nu_{n} \circ \sigma_{n+3}\right)$, para $n \geq 5$.

15. pág. 68 (7.11) $P\left(\nu_{5} \circ \sigma_{8}\right) \equiv \pm\left(\eta_{2} \circ \epsilon^{\prime}\right) \bmod \eta_{2}^{2} \circ \mu_{4}$ e $\Sigma \pi_{12}^{2}=\left[\eta_{3}^{2} \circ \mu_{5}\right] \approx \mathbb{Z} / 2$.

16. pág. $68(7.12) \epsilon_{3} \circ \nu_{11}=\nu^{\prime} \circ \bar{\nu}_{6}$ e $\epsilon^{\prime} \circ \eta_{13}=\nu^{\prime} \circ \epsilon_{6}$.

17. pág. $69(7.14) 2 \zeta_{5}= \pm \Sigma^{2} \mu^{\prime}$ e $\eta_{n}^{2} \circ \mu_{n+2}=4 \zeta_{n}$, para $n \geq 5$.

18. pág. $97(\operatorname{Lema} 10.2) H(\bar{\epsilon}) \equiv \nu_{5} \circ \sigma_{8} \circ \nu_{15} \bmod \nu_{5} \circ \eta_{8} \circ \mu_{9}$ e $2 \overline{\epsilon_{n}}=2 \bar{\epsilon}=0$ para $n \geq 3$.

19. pág. 107 (Lema 10.7) $\epsilon_{n} \circ \sigma_{n+8}=0$, para $n \geq 3, \sigma_{n} \circ \epsilon_{n+7}=0$, para $n \geq 11 \mathrm{e}$ $\bar{\nu}_{n} \circ \sigma_{n+8}=0$, para $n \geq 6$.

20. pág. 136 (Lema 12.2) $H\left(\bar{\mu}_{3}\right)=\rho^{I V}$ e $2 \bar{\mu}_{n}=2 \bar{\mu}=0$, para $n \geq 3$.

21. pág. 136 (Lema 12.3) $2 \epsilon^{\prime}=\eta_{3}^{2} \circ \bar{\epsilon}_{5}$, e $\Sigma \bar{\epsilon}^{\prime}=\Sigma \nu^{\prime} \circ \kappa_{7}$. 
22. pág. 137 (Lema 12.4) $H\left(\bar{\mu}^{\prime}\right) \equiv \bar{\mu}_{5} \bmod \Sigma^{3} \pi_{19}^{2}, H\left(\bar{\zeta}_{5}\right)=8 \rho^{\prime}, 2 \bar{\mu}^{\prime}=\eta_{3}^{2} \circ \bar{\mu}_{5}$, e $2 \bar{\zeta}_{5}=\Sigma^{2} \bar{\mu}^{\prime}$.

23. pág. 143 (Lema 12.10) $\nu_{n} \circ \sigma_{n+3} \circ \nu_{n+10}^{2}=\eta_{n} \circ \bar{\epsilon}_{n+1}$, para $n \geq 5$ e $\epsilon_{n} \circ \epsilon_{n+8}=$ $\epsilon_{n} \circ \bar{\nu}_{n+8}=\eta_{n} \circ \bar{\epsilon}_{n+1}=\bar{\epsilon}_{n} \circ \eta_{n+15}$, para $n \geq 3$.

24. pág. 161 (Prop. 12.20) $\sigma_{n} \circ \mu_{n+7}=\mu_{n} \circ \sigma_{n+9}=\eta_{n} \circ \rho_{n+1}=\rho_{n} \circ \eta_{n+16}$, para $n \geq 13$ e $\eta_{12} \circ \rho_{13}=\sigma_{12} \circ \mu_{19}$.

Aqui apresentamos os grupos de homotopia de $S^{3}$ calculados por Toda [46].

\begin{tabular}{|c|c|}
\hline $\mathrm{k}$ & $\pi_{k+3}\left(S^{3}\right)$ \\
\hline 1 & $\mathbb{Z} / 2$ \\
\hline 2 & $\mathbb{Z} / 2$ \\
\hline 3 & $\mathbb{Z} / 12$ \\
\hline 4 & $\mathbb{Z} / 2$ \\
\hline 5 & $\mathbb{Z} / 2$ \\
\hline 6 & $\mathbb{Z} / 3$ \\
\hline 7 & $\mathbb{Z} / 15$ \\
\hline 8 & $\mathbb{Z} / 2$ \\
\hline 9 & $\mathbb{Z} / 2 \oplus \mathbb{Z} / 2$ \\
\hline 10 & $\mathbb{Z} / 12 \oplus \mathbb{Z} / 2$ \\
\hline
\end{tabular}

\begin{tabular}{|c|c|}
\hline $\mathrm{k}$ & $\pi_{k+3}\left(S^{3}\right)$ \\
\hline 11 & $\mathbb{Z} / 84 \oplus \mathbb{Z} / 2 \oplus \mathbb{Z} / 2$ \\
\hline 12 & $\mathbb{Z} / 2 \oplus \mathbb{Z} / 2$ \\
\hline 13 & $\mathbb{Z} / 6$ \\
\hline 14 & $\mathbb{Z} / 30$ \\
\hline 15 & $\mathbb{Z} / 30$ \\
\hline 16 & $\mathbb{Z} / 6 \oplus \mathbb{Z} / 2$ \\
\hline 17 & $\mathbb{Z} / 12 \oplus \mathbb{Z} / 2 \oplus \mathbb{Z} / 2$ \\
\hline 18 & $\mathbb{Z} / 12 \oplus \mathbb{Z} / 2 \oplus \mathbb{Z} / 2$ \\
\hline 19 & $\mathbb{Z} / 132 \oplus \mathbb{Z} / 2$ \\
\hline
\end{tabular}

Em [32] Mimura e Toda continuam os cálculos dos grupos de homotopia das esferas e obtêm

$$
\pi_{23}\left(S^{3}\right) \approx \mathbb{Z} / 2 \oplus \mathbb{Z} / 2
$$

Já em [29] e [30] Mimura vai um pouco além e obtêm

$$
\begin{gathered}
\pi_{24}\left(S^{3}\right) \approx \mathbb{Z} / 2, \\
\pi_{25}\left(S^{3}\right) \approx \mathbb{Z} / 210 .
\end{gathered}
$$

Em 1975, Mimura, Mori e Oda [31] determinaram as 2-componentes dos grupos de homotopia das esferas $\pi_{n+i}\left(S^{n}\right)$, com $n \geq 2$ e $i=23,24$. Assim, temos

$$
\begin{gathered}
\pi_{26}\left(S^{3} ; 2\right) \approx \mathbb{Z} / 4, \\
\pi_{27}\left(S^{3} ; 2\right) \approx \mathbb{Z} / 2 \oplus \mathbb{Z} / 2 \oplus \mathbb{Z} / 2 .
\end{gathered}
$$

Quanto ao produto de Whitehead, encontramos em [3] III.1 as seguintes propriedades

$$
\begin{array}{ll}
{\left[\iota_{n}, \iota_{n}\right]=0} & n=1,3,7, \\
2\left[\iota_{n}, \iota_{n}\right]=0, & n \text { ímpar, } \\
{\left[\iota_{n}, \iota_{n}\right] \text { ordem infinita }} & n \text { par. }
\end{array}
$$

Também, em [17] pág. 330, podemos encontrar

$$
\left[\iota_{4}, \iota_{4}\right]=2 \nu_{4}+\Sigma \xi,
$$


com

$$
\pi_{6}\left(S^{3}\right)=\mathbb{Z} / 12[\xi]
$$

e

$$
\pi_{7}\left(S^{4}\right)=\mathbb{Z} / 12[\Sigma \xi] \oplus \mathbb{Z}\left[\nu_{4}\right]
$$

Temos o diagrama

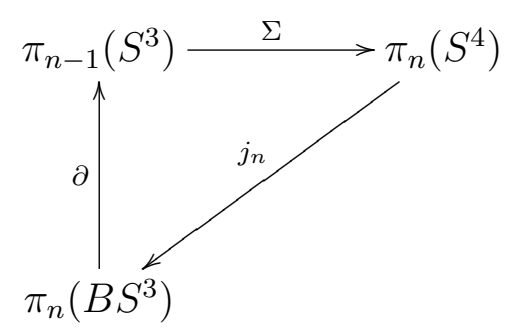

e $j_{n} \psi=1$, com $\psi=\Sigma \partial$. Logo, $j_{n}$ é sobrejetiva e $\psi$ é injetiva.

Se $n=4, \Sigma$ é um isomorfismo e, com isso, $j_{4}$ é um isomorfismo. Assim, $\pi_{4}\left(B S^{3}\right)=$ $\mathbb{Z}\left[j_{4}\left(\iota_{4}\right)=\partial^{-1}\left(\iota_{3}\right)\right]$.

Queremos calcular

$$
j_{7}\left(\left[\iota_{4}, \iota_{4}\right]\right)=\left[j_{4}\left(\iota_{4}\right), j_{4}\left(\iota_{4}\right)\right]
$$

Usando a relação acima,

$$
\left[j_{4}\left(\iota_{4}\right), j_{4}\left(\iota_{4}\right)\right]=2 j_{7}\left(\nu_{4}\right)+j_{7}(\Sigma \xi)
$$

Agora, temos o isomorfismo de Serre

$$
\begin{gathered}
\pi_{6}\left(S^{3}\right) \oplus \pi_{7}\left(S^{7}\right) \rightarrow \pi_{7}\left(S^{4}\right), \\
(a, b) \mapsto \Sigma a+\nu_{4} \circ b .
\end{gathered}
$$

Como $\pi_{7}\left(S^{7}\right)=\mathbb{Z}\left[\iota_{7}\right], b=n \iota_{7}$, então $\nu_{4} \circ b=\nu_{4} \circ\left(n \iota_{7}\right)=n \nu_{4} \circ \iota_{7}$, já que $\iota_{7}$ é uma suspensão.

Como a decomposição acima é uma soma direta, segue que $\nu_{4} \in$ Nuc $j_{7}$. Então

$$
\left[j_{4}\left(\iota_{4}\right), j_{4}\left(\iota_{4}\right)\right]=j_{7}(\Sigma \xi)=\partial^{-1}(\xi)
$$

Devemos ter cuidado e observar que

$$
\partial\left(\left[j_{4}\left(\iota_{4}\right), j_{4}\left(\iota_{4}\right)\right]\right) \neq\left[\partial j_{4}\left(\iota_{4}\right), \partial j_{4}\left(\iota_{4}\right)\right]=\left[\iota_{3}, \iota_{3}\right]=0
$$

De fato, em geral

$$
\partial[x, y] \neq[\partial(x), \partial(y)]
$$

já que $\partial$ não é induzida por uma aplicação.

Lema 4.4.1. Nuc $j_{n}=\nu_{4} \circ \pi_{n}\left(S^{7}\right)$ e a restrição de $j_{n}$ é injetiva sobre $\Sigma \pi_{n-1}\left(S^{3}\right)$ em $\pi_{n}\left(S^{4}\right)$. Em particular, $j_{n}\left(\nu_{4} \circ y\right)=0$. 
Demonstração: Pelo isomorfismo de Serre,

$$
\pi_{n}\left(S^{4}\right)=\Sigma \pi_{n-1}\left(S^{3}\right) \oplus \nu_{4} \circ \pi_{n}\left(S^{7}\right) .
$$

Por outro lado, do diagrama de homomorfismos de grupos abelianos

$$
\pi_{n}\left(B S^{3}\right) \underset{\partial}{\longrightarrow} \pi_{n-1}\left(S^{3}\right) \underset{\Sigma}{\longrightarrow} \pi_{n}\left(S^{4}\right) \underset{j_{n}}{\longrightarrow} \pi_{n}\left(B S^{3}\right)
$$

cuja composição é a identidade, $j_{n} \Sigma \partial=1$, segue que

$$
\pi_{n}\left(S^{4}\right)=\operatorname{Nuc} j_{n} \oplus \operatorname{Im} \Sigma \partial=\Sigma \pi_{n-1}\left(S^{3}\right) \oplus \operatorname{Nuc} j_{n} .
$$

Como a decomposição é uma soma direta, o resultado segue. 


\section{Trocando a esfera $S^{n}$ pelo espaço projetivo quaterniônico}

Consideremos aqui os fibrados principais sobre o espaço projetivo quaterniônico.

Assim como no caso de $S^{n}$, uma pergunta natural é saber quantos fibrados linhas quaterniônicos existem sobre o espaço $\mathbb{H} P^{2}$. Essa pergunta foi respondida em [27], no qual podemos encontrar o seguinte resultado:

Teorema 4.4.2. $\left[H P^{2}, B S^{3}\right]=R_{2,+} \times P(2) \cup R_{2,-} \times Q(2)$, onde $R_{2}=\{n \in \mathbb{Z} \mid n=$ $0,1,9,16(\bmod 24)\}$.

Aqui, +/- denotam subconjuntos par/ímpar de um subconjunto de números inteiros e $P(2), Q(2)$ são conjuntos de cardinalidade 1,2 , respectivamente.

Como no caso da esfera, assumimos $f$ como a aplicação que classifica o fibrado sobre $\mathrm{H} P^{2}$ e denotamos por $\mathcal{G}_{f}$ o grupo de gauge desse fibrado. Temos as equivalências de homotopia [39]:

$$
\begin{aligned}
& \mathcal{G}_{f} \sim \Omega m\left(\mathbb{H} P^{2}, B S^{3} ; f\right), \\
& B \mathcal{G}_{f} \sim m\left(\mathbb{H} P^{2}, B S^{3} ; f\right) .
\end{aligned}
$$

Consideremos a fibração avaliação

$$
e v: m\left(\mathbb{H} P^{2}, B S^{3} ; f\right) \rightarrow B S^{3},
$$

com ev $(g)=g\left(x_{0}\right)$, para todo $g \in m\left(\mathbb{H} P^{2}, B S^{3} ; f\right)$ e $x_{0}$ é o ponto-base de $\mathbb{H} P^{2}$. A seqüência de fibrações e inclusões de fibra (a menos de homotopia) fica:

$$
\begin{gathered}
\cdots \longrightarrow \mathcal{G}(f) \underset{\Omega e v}{\longrightarrow} \Omega_{f} B S^{3} \sim S^{3} \longrightarrow m_{0}\left(\mathbb{H} P^{2}, B S^{3} ; f\right) \longrightarrow \\
\longrightarrow m\left(\mathbb{H} P^{2}, B S^{3} ; f\right) \stackrel{e v}{\longrightarrow} B S^{3} .
\end{gathered}
$$

Assim, temos três fibrações

$$
\begin{gathered}
\mathcal{G}_{f} \longrightarrow S^{3} \longrightarrow m_{0}\left(\mathbb{H} P^{2}, B S^{3} ; f\right) \\
S^{3} \longrightarrow m_{0}\left(\mathbb{H} P^{2}, B S^{3} ; f\right) \longrightarrow m\left(\mathbb{H} P^{2}, B S^{3} ; f\right) \\
m_{0}\left(\mathbb{H} P^{2}, B S^{3} ; f\right) \longrightarrow m\left(\mathbb{H} P^{2}, B S^{3} ; f\right) \underset{e v}{\longrightarrow} B S^{3} .
\end{gathered}
$$


Considerando a primeira fibração, vemos que $\mathcal{G}_{f}$ é a fibra de homotopia de uma aplicação $h: S^{3} \rightarrow m_{0}\left(\mathbb{H} P^{2}, B S^{3} ; f\right)$. Isso significa que os diferentes tipos de $\mathcal{G}_{f}$ são dados por diferentes aplicações $h: S^{3} \rightarrow m_{0}\left(\mathbb{H} P^{2}, B S^{3} ; f\right)$.

Assim, estamos interessados nas possíveis classes de homotopia de aplicações $h: S^{3} \rightarrow$ $m_{0}\left(\mathbb{H} P^{2}, B S^{3} ; f\right)$, isto é, em $\pi_{3}\left(m_{0}\left(\mathbb{H} P^{2}, B S^{3} ; f\right)\right)$ já que, certamente, os diferentes tipos de homotopia dos grupos de gauge estão contidos nesse conjunto.

Para calcular tal grupo, seguimos a idéia desenvolvida em [27]: consideremos a inclusão natural $i_{n-1}: \mathbb{H} P^{n-1} \rightarrow \mathbb{H} P^{n}$, a qual induz à fibração (veja [23])

$$
\left(i_{n-1}\right)_{\sharp}: m_{0}\left(\mathbb{H} P^{n}, B S^{3}\right) \rightarrow m_{0}\left(\mathbb{H} P^{n-1}, B S^{3}\right)
$$

$\operatorname{com}\left(i_{n-1}\right)_{\sharp}(f)=f \circ i_{n-1}$ para todo $f \in m_{0}\left(\mathbb{H} P^{n}, B S^{3}\right)$. Seja $f_{\lambda}$ com grau $\lambda$. Denotemos por $m_{n}\left(f_{\lambda}\right)=m_{0}\left(\mathbb{H} P^{n}, B S^{3} ; f_{\lambda}\right)$ a componente de $f_{\lambda}$ e $M_{n}\left(f_{\lambda}\right)=\bigcup_{g}\left(m_{n}\left(f_{\lambda}\right)\right)$, sendo que $g$ percorre todas as aplicações em $m_{0}\left(\mathbb{H} P^{n}, B S^{3}\right)$ com grau $\lambda$.

Se $p_{n}$ é a restrição de $\left(i_{n-1}\right)_{\sharp}$ a $M_{n}\left(f_{\lambda}\right)$, então sua fibra $F_{n}\left(f_{\lambda}\right)$ sobre $f_{\lambda} i_{n-1}$, com ponto base $f_{\lambda}$, tem o tipo de homotopia de $\Omega^{4 n} B S^{3}$, e temos a seqüência exata

$$
\cdots \underset{\partial_{n, q+1}}{\longrightarrow} \pi_{q}\left(F_{n}\left(f_{\lambda}\right)\right) \underset{j_{n, q}}{\longrightarrow} \pi_{q}\left(M_{n}\left(f_{\lambda}\right)\right) \underset{p_{n, q}}{\longrightarrow} \pi_{q}\left(M_{n-1}\left(f_{\lambda} i_{n-1}\right)\right) \underset{\partial_{n, q}}{\longrightarrow} \cdots
$$

As seqüências com índices $n-1$ e $n$ podem ser compostas do seguinte modo:

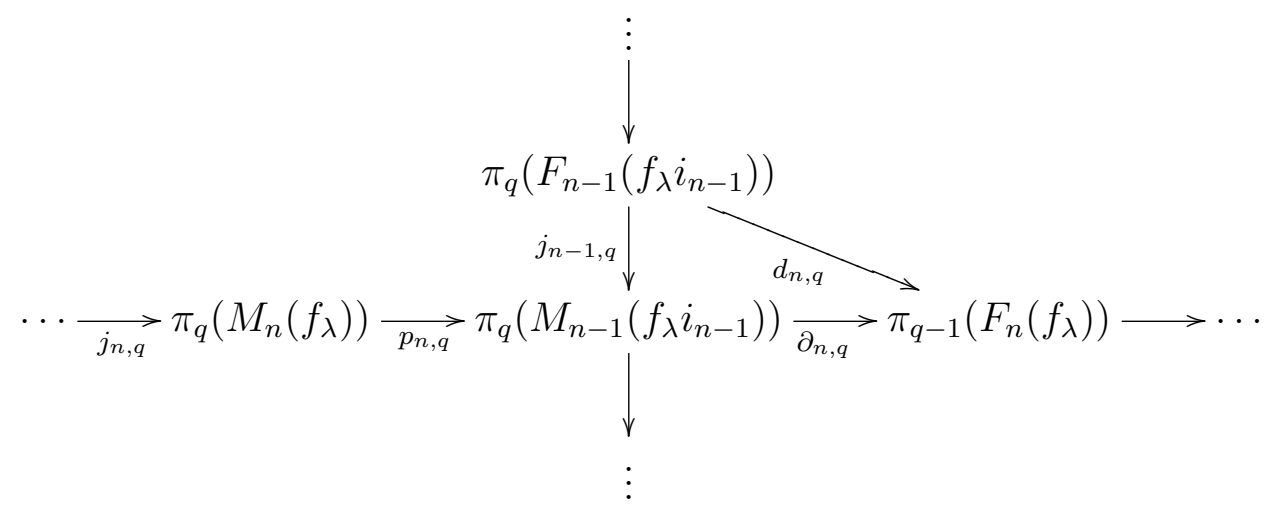

Aqui, uma descrição explícita do homomorfismo $d_{n, q}=\partial_{n, q} j_{n-1, q}$ pode ser obtida usando a construção de James [22], como em [19]:

$$
\begin{gathered}
d_{n, q}: \pi_{q}\left(F_{n-1}\left(f_{\lambda} i_{n-1}\right)\right) \approx \pi_{4 n+q-4}\left(B S^{3}\right) \rightarrow \pi_{q-1}\left(F_{n}\left(f_{\lambda}\right)\right) \approx \pi_{4 n+q-1}\left(B S^{3}\right), \\
d_{n, q}: \zeta \mapsto \pm(n-1) \zeta \circ j_{*}\left(\nu_{4 n+q-4}\right) \pm \lambda[\gamma, \zeta],
\end{gathered}
$$

sendo $\gamma=[j]$ a classe da inclusão $j: S^{4} \rightarrow B S^{3}$. Em particular, o homomorfismo

$$
j_{1, q}: \pi_{q}\left(F_{1}\left(f_{\lambda}\right)\right) \approx \pi_{q+4}\left(B S^{3}\right) \rightarrow \pi_{q}\left(M_{1}\left(f_{\lambda}\right)\right)
$$

é sempre um isomorfismo, já que $M_{0}\left(f_{\lambda} i_{0}\right)$ é apenas um ponto. 
Quando $n=2$, obtemos o seguinte diagrama:

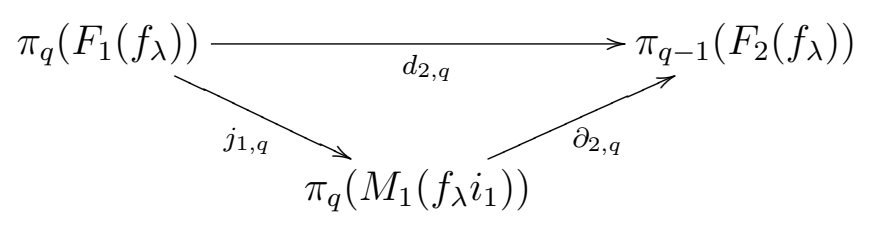

e, como $j_{1, q}$ é um isomorfismo, identificamos $d_{2, q}$ com $\partial_{2, q}$. Assim, podemos calcular o homomorfismo bordo

$$
\begin{gathered}
\partial_{2, q}=d_{2, q}: \pi_{q}\left(F_{1}\left(f_{\lambda}\right)\right) \approx \pi_{q+4}\left(B S^{3}\right) \rightarrow \pi_{1}\left(F_{2}\left(f_{\lambda}\right)\right) \approx \pi_{q+7}\left(B S^{3}\right), \\
\partial_{2, q}(\zeta)= \pm \zeta \circ j_{*}\left(\nu_{q+4}\right) \pm \lambda[\gamma, \zeta]= \pm \zeta \circ j_{*}\left(\nu_{q+4}\right) \pm \lambda\left[j_{*}\left(\iota_{4}\right), \zeta\right] .
\end{gathered}
$$

Usando a composição $S^{4} \underset{i d}{\longrightarrow} S^{4} \underset{j_{1}}{\longrightarrow} B S^{3}$, obtemos $j_{1 *}\left(\iota_{4}\right)=\gamma$.

Consideremos a parte final da seqüência exata de homotopia:

$$
\begin{gathered}
\cdots \longrightarrow \pi_{4}\left(M_{1}\left(f_{\lambda} i_{1}\right)\right) \approx \pi_{8}\left(B S^{3}\right) \underset{\partial_{2,4}}{\longrightarrow} \\
\underset{\partial_{2,4}}{\longrightarrow} \pi_{3}\left(F_{2}\left(f_{\lambda}\right)\right) \approx \pi_{11}\left(B S^{3}\right) \underset{j_{2,3}}{\longrightarrow} \pi_{3}\left(M_{2}\left(f_{\lambda}\right)\right) \underset{p_{2,3}}{\longrightarrow} \pi_{3}\left(M_{1}\left(f_{\lambda} i_{1}\right)\right) \approx \pi_{7}\left(B S^{3}\right) \underset{\partial_{2,3}}{\longrightarrow} \underset{\partial_{2,3}}{\longrightarrow} \pi_{2}\left(F_{2}\left(f_{\lambda}\right)\right) \approx \pi_{10}\left(B S^{3}\right) \underset{j_{2,2}}{\longrightarrow} \pi_{2}\left(M_{2}\left(f_{\lambda}\right)\right) \underset{p_{2,2}}{\longrightarrow} \pi_{2}\left(M_{1}\left(f_{\lambda} i_{1}\right)\right) \approx \pi_{6}\left(B S^{3}\right) \underset{\partial_{2,2}}{\longrightarrow} \underset{\partial_{2,2}}{\longrightarrow} \pi_{1}\left(F_{2}\left(f_{\lambda}\right)\right) \approx \pi_{9}\left(B S^{3}\right) \underset{j_{2,1}}{\longrightarrow} \pi_{1}\left(M_{2}\left(f_{\lambda}\right)\right) \underset{p_{2,1}}{\longrightarrow} \pi_{1}\left(M_{1}\left(f_{\lambda} i_{1}\right)\right) \approx \pi_{5}\left(B S^{3}\right) \underset{\partial_{2,1}}{\longrightarrow} \\
\underset{\partial_{2,1}}{\longrightarrow} \pi_{0}\left(F_{2}\left(f_{\lambda}\right)\right) \approx \pi_{8}\left(B S^{3}\right) \underset{j_{2,0}}{\longrightarrow} \pi_{0}\left(M_{2}\left(f_{\lambda}\right)\right) \underset{p_{2,0}}{\longrightarrow} \pi_{0}\left(M_{1}\left(f_{\lambda} i_{1}\right)\right) .
\end{gathered}
$$

Disso, obtemos a seqüência

$$
\begin{gathered}
\cdots \longrightarrow \mathbb{Z} / 2\left[j_{8}\left(\Sigma \nu^{\prime} \circ \eta_{7}\right)\right] \underset{\partial_{2,4}}{\longrightarrow} \mathbb{Z} / 15\left[j_{11}(\Sigma z)\right] \underset{j_{2,3}}{\longrightarrow} \\
\underset{j_{2,3}}{\longrightarrow} \pi_{3}\left(M_{2}\left(f_{\lambda}\right)\right) \underset{p_{2,3}}{\longrightarrow} \mathbb{Z} / 12\left[j_{7}(\Sigma \xi)\right] \underset{\partial_{2,3}}{\longrightarrow} \\
\underset{\partial_{2,3}}{\longrightarrow} \mathbb{Z} / 3\left[j_{10}\left(\Sigma\left(\alpha_{1}(3 ; 3) \circ \alpha_{1}(6 ; 3)\right)\right)\right] \underset{j_{2,2}}{\longrightarrow} \pi_{2}\left(M_{2}\left(f_{\lambda}\right)\right) \underset{p_{2,2}}{\longrightarrow} \mathbb{Z} / 2\left[j_{6}\left(\eta_{4}^{2}\right)\right] \underset{\partial_{2,2}}{\longrightarrow} \\
\underset{\partial_{2,2}}{\longrightarrow} \mathbb{Z} / 2\left[j_{9}\left(\Sigma \nu^{\prime} \circ \eta_{7}^{2}\right)\right] \underset{j_{2,1}}{\longrightarrow} \pi_{1}\left(M_{2}\left(f_{\lambda}\right)\right) \underset{p_{2,1}}{\longrightarrow} \mathbb{Z} / 2\left[j_{5}\left(\eta_{4}\right)\right] \underset{\partial_{2,1}}{\longrightarrow} \\
\underset{\partial_{2,1}}{\longrightarrow} \mathbb{Z} / 2\left[j 8\left(\Sigma \nu^{\prime} \circ \eta_{7}\right)\right] \underset{j_{2,0}}{\longrightarrow} \pi_{0}\left(M_{2}\left(f_{\lambda}\right)\right) \underset{p_{2,0}}{\longrightarrow} 0 .
\end{gathered}
$$

com o seguinte homomorfismo fazendo a conexão

$$
\begin{gathered}
d_{2,1}=\partial_{2,1}: \mathbb{Z} / 2\left[j_{5}\left(\eta_{4}\right)\right] \rightarrow \mathbb{Z} / 2\left[j_{8}\left(\Sigma \nu^{\prime} \circ \eta_{7}\right)\right] \\
\partial_{2,1}(\zeta)=\zeta \circ j_{8}\left(\nu_{5}\right)+\lambda \circ\left[j_{4}\left(\iota_{4}\right), \zeta\right],
\end{gathered}
$$

que, usando [46] 5.9 pág. 44

$$
\eta_{3} \circ \nu_{4}=\nu^{\prime} \circ \eta_{6}
$$

e

$$
\eta_{4} \circ \nu_{5}=\Sigma\left(\eta_{3} \circ \nu_{4}\right)=\Sigma\left(\nu^{\prime} \circ \eta_{6}\right)=\Sigma \nu^{\prime} \circ \eta_{7} .
$$


Temos

$$
\partial_{2,1}\left(j_{5}\left(\eta_{4}\right)\right)=((\lambda+1) \bmod 2) j_{8}\left(\Sigma \nu^{\prime} \circ \eta_{7}\right)
$$

e

$$
\begin{gathered}
d_{2,2}=\partial_{2,2}: \mathbb{Z} / 2\left[j_{6}\left(\eta_{4}^{2}\right)\right] \rightarrow \mathbb{Z} / 2\left[j_{9}\left(\Sigma \nu^{\prime} \circ \eta_{7}^{2}\right)\right] \\
\partial_{2,2}(\zeta)=\zeta \circ j_{9}\left(\nu_{6}\right)+\lambda \circ\left[j_{4}\left(\iota_{4}\right), \zeta\right],
\end{gathered}
$$

que, usando [46] 5.9 pág. 44, nos dá

$$
\eta_{4}^{2} \circ \nu_{6}=\eta_{4} \circ \eta_{5} \circ \nu_{6}=0,
$$

e, por [47] Capítulo X 8.18 pág. 484, temos

$$
\begin{gathered}
{\left[j_{4}\left(\iota_{4}\right), j_{6}\left(\eta_{4}^{2}\right)\right]=j_{9}\left(\left[\iota_{4}, \Sigma\left(\eta_{3} \circ \eta_{4}\right)\right]\right)=j_{9}\left(\left[\iota_{4}, \iota_{4}\right] \circ \Sigma^{4}\left(\eta_{3} \circ \eta_{4}\right)\right)=} \\
=j_{9}\left(\left(2 \nu_{4} \pm \Sigma \nu^{\prime}\right) \circ \eta_{7} \circ \eta_{8}\right)=j_{9}\left(\Sigma \nu^{\prime} \circ \eta_{7} \circ \eta_{8}\right),
\end{gathered}
$$

e obtemos

$$
\partial_{2,2}\left(j_{6}\left(\eta_{4}^{2}\right)\right)=\lambda j_{9}\left(\Sigma \nu^{\prime} \circ \eta_{7}^{2}\right)
$$

Também,

$$
\begin{gathered}
d_{2,3}=\partial_{2,3}: \mathbb{Z} / 12\left[j_{7}(\Sigma \xi)\right] \rightarrow \mathbb{Z} / 3\left[j_{10}\left(\Sigma\left(\alpha_{1}(3 ; 3) \circ \alpha_{1}(6 ; 3)\right)\right)\right] \\
\partial_{2,3}(\zeta)=\zeta \circ j_{10}\left(\nu_{7}\right)+\lambda \circ\left[j_{4}\left(\iota_{4}\right), \zeta\right] \\
\partial_{2,3}\left(j_{7}(\Sigma \xi)\right)=j_{7}(\Sigma \xi) \circ j_{10}\left(\nu_{7}\right)+\lambda\left[j_{4}\left(\iota_{4}\right), j_{7}(\Sigma \xi)\right] .
\end{gathered}
$$

Por [46] 4.7 e usando o fato que $\nu^{\prime} \circ \nu_{6} \in \pi_{9}^{3}=0$ ([46] pág. 46),

$$
\Sigma \xi \circ \nu_{7}=\Sigma\left(\xi \circ \nu_{6}\right)=\Sigma\left(\nu^{\prime} \circ \nu_{6}\right)=0,
$$

e por [47] Capítulo X 8.18 pág. 484,

$$
\begin{gathered}
{\left[\iota_{4}, \Sigma \xi\right]=\left[\iota_{4}, \iota_{4}\right] \circ \Sigma^{4} \xi=\left[\iota_{4}, \iota_{4}\right] \circ \Sigma^{4} \nu^{\prime}=} \\
\left(2 \nu_{4} \pm \Sigma \nu^{\prime}\right) \circ \Sigma^{4} \nu^{\prime}=2 \nu_{4} \circ \Sigma^{4} \nu^{\prime}
\end{gathered}
$$

já que $\Sigma \nu^{\prime} \circ \Sigma^{4} \nu^{\prime}=\Sigma\left(\nu^{\prime} \circ \Sigma^{3} \nu^{\prime}\right)$ e $\nu^{\prime} \circ \Sigma^{3} \nu^{\prime} \in \pi_{9}^{3}=0$.

Então, temos

$$
\begin{aligned}
\partial_{2,3}\left(j_{7}(\Sigma \xi)\right)= & j_{7}(\Sigma \xi) \circ j_{10}\left(\nu_{7}\right)+\lambda\left[j_{4}\left(\iota_{4}\right), j_{7}(\Sigma \xi)\right]= \\
& \lambda j_{10}\left(2 \nu_{4} \circ \Sigma^{4} \nu^{\prime}\right)=0,
\end{aligned}
$$

pois $\nu_{4} \in \operatorname{Nuc} j_{10}$.

Agora,

$$
\begin{gathered}
d_{2,4}=\partial_{2,4}: \mathbb{Z} / 2\left[j_{8}\left(\Sigma \nu^{\prime} \circ \eta_{7}\right)\right] \rightarrow \mathbb{Z} / 15\left[j_{11}(\Sigma z)\right] \\
\partial_{2,4}(\zeta)=\zeta \circ j_{11}\left(\nu_{8}\right)+\lambda \circ\left[j_{4}\left(\iota_{4}\right), \zeta\right], \\
\partial_{2,4}\left(j_{8}\left(\Sigma \nu^{\prime} \circ \eta_{7}\right)\right)=j_{8}\left(\Sigma \nu^{\prime} \circ \eta_{7}\right) \circ j_{11}\left(\nu_{8}\right)+\lambda\left[j_{4}\left(\iota_{4}\right), j_{8}\left(\Sigma \nu^{\prime} \circ \eta_{7}\right)\right]=0,
\end{gathered}
$$

já que, usando [46] 5.9 pág. 44

$$
\Sigma \nu^{\prime} \circ \eta_{7} \circ \nu_{8}=0
$$

e por [47] Capítulo X 8.18 pág. 484 e [46] 4.7 pág. 36

$$
\left[\iota_{4}, \iota_{4}\right] \circ \Sigma^{3}\left(\Sigma \nu^{\prime} \circ \eta_{7}\right)=\left[\iota_{4}, \iota_{4}\right] \circ \Sigma^{4}\left(\nu^{\prime} \circ \eta_{6}\right) \in \pi_{11}^{4}=0 .
$$

Podemos afirmar o seguinte 
1. se $\lambda$ é par, então $\partial_{2,1}$ é injetiva e assim é um isomorfismo. Então Nuc $j_{2,0}=\operatorname{Im} \partial_{2,1}=$ $\mathbb{Z} / 2$ e, como $j_{2,0}$ é sobrejetiva, já que $p_{2,0}$ é trivial, isso significa que $\pi_{0}\left(M_{2}\left(f_{\lambda}\right)\right)=0$.

2. se $\lambda$ é ímpar, então $\partial_{2,1}$ é trivial e, logo, Nuc $j_{2,0}=0$. Assim $j_{2,0}$ é injetiva e $\operatorname{Im} j_{2,0}=\operatorname{Nuc} p_{2,0}=\mathbb{Z} / 2$, mas como $p_{2,0}$ é trivial, isso implica $\pi_{0}\left(M_{2}\left(f_{\lambda}\right)\right) \approx \mathbb{Z} / 2$.

3. se $\lambda$ é par, então $\partial_{2,1}$ é injetiva, logo Nuc $\partial_{2,1}=0=\operatorname{Im} p_{2,1}$ e $p_{2,1}$ é trivial e $\pi_{1}\left(M_{2}\left(f_{\lambda}\right)\right)=$ Nuc $p_{2,1}=\operatorname{Im} j_{2,1}$. Mas, com $\lambda$ par, $\partial_{2,2}$ é trivial e, logo, Nuc $j_{2,1}=0$ e $\pi_{1}\left(M_{2}\left(f_{\lambda}\right)\right)=\operatorname{Im} j_{2,1} \approx \mathbb{Z} / 2$.

4. se $\lambda$ é ímpar, então $\partial_{2,1}$ é trivial e Nuc $\partial_{2,1}=\mathbb{Z} / 2=\operatorname{Im} p_{2,1}$. Por outro lado, $\partial_{2,2}$ é injetiva, então Nuc $j_{2,1}=\mathbb{Z} / 2=\operatorname{Im} \partial_{2,2}$, e isso implica que $j_{2,1}$ é trivial, Nuc $p_{2,1}=\operatorname{Im} j_{2,1}=0$, e com isso $\pi_{1}\left(M_{2}\left(f_{\lambda}\right)\right)=\operatorname{Im} p_{2,1} /$ Nuc $p_{2,1} \approx \mathbb{Z} / 2$.

5. se $\lambda$ é par, $\partial_{2,2}$ é trivial. Logo, $\operatorname{Im} p_{2,2}=\mathbb{Z} / 2$. Como $\partial_{2,3}=0$, temos que $j_{2,2}$ é injetiva, e disso $\operatorname{Im} j_{2,2}=\mathbb{Z} / 3=\operatorname{Nuc} p_{2,2}$. Assim, $\pi_{2}\left(M_{2}\left(f_{\lambda}\right)\right) / \operatorname{Nuc} p_{2,2}=\operatorname{Im} p_{2,2}=0$ e isso implica que $\pi_{2}\left(M_{2}\left(f_{\lambda}\right)\right) \approx \mathbb{Z} / 6$.

6. se $\lambda$ é ímpar, $\partial_{2,2}$ é injetiva. Logo, $\operatorname{Im~} p_{2,2}=0$. Já que $\partial_{2,3}=0$, temos que $j_{2,2}$ é injetiva, e assim $\operatorname{Im} j_{2,2}=\mathbb{Z} / 3=$ Nuc $p_{2,2}$. Com isso, $\pi_{2}\left(M_{2}\left(f_{\lambda}\right)\right) / \mathrm{Nuc} p_{2,2}=$ $\operatorname{Im} p_{2,2} \approx \mathbb{Z} / 2$ e isso implica $\pi_{2}\left(M_{2}\left(f_{\lambda}\right)\right) \approx \mathbb{Z} / 3$.

7. como $\partial_{2,4}=0$ e $\partial_{2,3}=0$, temos que $j_{2,3}$ é injetiva e, logo, $\operatorname{Im} j_{2,3}=\mathbb{Z} / 15=$ Nuc $p_{2,3}$, e $\operatorname{Im} p_{2,3}=\operatorname{Nuc} \partial_{2,3}=\mathbb{Z} / 12$. Assim, $\pi_{3}\left(M_{2}\left(f_{\lambda}\right)\right) /$ Nuc $p_{2,3}=\operatorname{Im} p_{2,3} \approx \mathbb{Z} / 12$, o que implica $\pi_{3}\left(M_{2}\left(f_{\lambda}\right)\right) \approx \mathbb{Z} / 15 \oplus \mathbb{Z} / 12$.

Portanto, pelo ítem 7, temos que os diferentes tipos de homotopia dos grupos de gauge serão, no máximo, de 180 tipos. 


\section{Referências Bibliográficas}

[1] Adams, J.F., On the non-existence of elements of Hopf invariant one, Ann. of Math. (2) 72 (1960), 20-104.

[2] Atiyah, M.F.; Bott, R., The Yang-Mills equations over Riemann surfaces, Philos. Trans. Roy. Soc. London A 308 (1982) 523-615.

[3] Baus, H.J., Commutator calculus and groups of homotopy classes, LNM 50 (1981).

[4] Bourbaki, N., Topologie gènèrale II, Hermann et Co., Paris 1961.

[5] Booth, P.; Heath, P.; Morgan, C.; Piccinini, R., H-spaces of self-equivalences of fibrations and bundles, Proc. London Math. Soc. 49 (1984) 111-127.

[6] Bott, R.; Tu, L. W., Differential forms in algebraic topology, Springer-Verlag, New York-Heidelberg-Berlin, 1982.

[7] Bredon, G. E., Topology and geometry, Graduate Texts in Mathematics ; 139, New York : Springer, 1993.

[8] Cartan, H., Suspension et invariant de Hopf, Exposé 5, Séminaire H. Cartan, 11e. anneé, 1958/59. Invariant de Hopf et opérations cohomologiques secondaires. École Normale Supérieure, Paris, 1959.

[9] Crabb, M.C.; Sutherland,W.A., Counting homotopy types of gauge groups, Proc. London Math. Soc. 81 (2000) 747-768.

[10] Davis, J.F.; Kirk, P., Lecture notes in algebraic topology, American Mathematical Society, 2001.

[11] Diek, T., Transformation groups, W. de Gruyter, Berlin - New York 1987.

[12] Freudenthal, H., Uber die klassen de sphärenabbildungen, Comp. Math., 5 (1937).

[13] Fritsch, R.; Piccinini, R., Cellular structures in topology, Cambridge U. Press, Cambridge 1990.

[14] Hamanaka, H.; Kono, A., Unstable $K^{1}$-group and homotopy type of certain gauge groups, Proc. Roy. Soc. Edinburgh Sect. A 136 (2006), no. 1, 149-155.

[15] Hamanaka, H.; Kono, A., Homotopy type of gauge groups of $S U(3)$-bundles over $S^{6}$, Topology and App. 154 (2007) 1377-1380. 
[16] Hopf, H., Uber die abbildungen von sphären aufsphären niedrigerer dimension, Fund. Math. 25 (1935).

[17] Hu, S-T., homotopy theory, Ac. Press (1959).

[18] Husemoller, D., Fibre bundles, McGraw-Hill, 1966.

[19] Iwase, N.; Maruyama, K.; Oka, S., A note on $\mathcal{E}\left(\mathbb{H} P^{n}\right)$ for $n \leq 4$, Math. J. Okayama Univ. 33 (1991) p. 163-176.

[20] James, I.M., Reduced product spaces, Ann. de Math., 62 (1955), 170-197.

[21] James, I.M., Suspension triad of a sphere, Ann. of Math., 63 (1956), 407-429.

[22] James, I.M., Note on cup products, Proc. Amer. Math. Soc. 84 (1957) p. 374-383.

[23] James, I.M., General topology an homotopy theory, Springer-Verlag New York, 1984.

[24] Koh, S.S., Note on the homotopy properties of the components of the mapping space $X^{S^{p}}$, Proc. Amer. Math. Soc., 11 (1960), 896-904.

[25] Kono, A., A note on the homotopy type of certain gauge groups, Proc. Royal Soc. Edinburgh, 117A (1991), 295-297.

[26] Kono, A.; Tsukuda, S., A remark on the homotopy type of certain gauge groups, J. Math. Kyoto Univ., 36-1 (1996), 115-121.

[27] Lima Gonçalves, D.; Spreafico, M., Quaternionic line bundles over quaternionic projective spaces (2006) to appear in Math. J. Okayama Univ.

[28] Miller, The topology of rotation groups, Annals of Math. 57 (1953), 90-111.

[29] Mimura, M., On the generalized Hopf homomorphism and the higher composition, Part I, J. Math. Kyoto Univ. 4-1 (1964) 171-190.

[30] Mimura, M., On the generalized Hopf homomorphism and the higher composition. Part II. $\pi_{n+i}\left(S^{n}\right)$ for $i=21$ and 22., J. Math. Kyoto Univ. 4-2 (1965) 301-326.

[31] Mimura, M.; Mori, M.; Oda, N., Determination of 2-components of the 23 and 24stems in homotopy groups of spheres, Memoirs of the Faculty of Science, Kyushu University, Ser. A. Vol. 29, No. 1, 1975.

[32] Mimura, M.; Toda, H., The $(n+20)$-th homotopy groups of $n$-spheres, J. Math. Kyoto Univ. 3-1 (1963) 37-58.

[33] Mimura, M.; Toda, H., Topology of Lie groups, Transl. of Math. Mon. 91 (1978).

[34] Morgan, C.; Piccinini, R., Conjugacy classes of groups of bundles automorphisms, Manscripta Math. 63 (1989), 233-244.

[35] Munkres, J.R., Elements of algebraic topology, The Benjamin/Cummings Publishing Company, Inc. (1984). 
[36] Oka, S.; Sawashita, N.; Sugawara, M., On the groups of self equivalences of a mapping cone, Hiroshima Math. J. 4 (1974) 9-28.

[37] Piccinini, R.A., Lectures on homotopy theory. Università Degli Studi di Milano, Milano, Italia.

[38] Piccinini, R.; Spreafico, M., Conjugacy classes of gauge groups, Queen's papers in pure and applied mathematics 111 (1998) Kingston.

[39] Piccinini, R.; Spreafico, M., The Milgram-Steenrod construction of classifying spaces for topological groups, Expo. Math. (1998).

[40] Serre, J.P., Homologie singulière de espaces fibrés, Ann. of Math., 54 (1951), 425-505.

[41] Serre, J.P., Groups d'homotopie et groupes abéliens, Ann. of Math., 58 (1953), 258294.

[42] Spanier, E., Algebrairc topology, Springer-Verlag New York, Inc. 1966.

[43] Steenrod, N.E., Topology of fibre bundles, Princeton.

[44] Toda, H., On double suspension $E^{2}$, Jour. of. Inst. Ply. Univ., 7 (1956), 103-145.

[45] Toda, H., p-primary components of homotopy groups IV, Compositions and toric constructions Memoirs. Univ. of Kyoto, 32 (1959), 297-332.

[46] Toda, H., Composition methods in the homotopy groups of the spheres, Ann. of Math. Studies No. 49 Princeton, 1962.

[47] Whitehead, G., Elements of homotopy theory, Springer Verlag, New York, 1978.

[48] Whitehead, G., The $(n+2)^{\text {nd }}$ homotopy groups of the $n$-spheres, Ann. of Math. Soc., 52 (1950) 245-247. 


\section{Tabela de símbolos}

\begin{tabular}{|c|c|}
\hline$S^{n}$ & Esfera unitária $n$-dimensional em $\mathbb{R}^{n+1}$ \\
\hline Top & Categoria dos espaços topológicos e aplicações contínuas \\
\hline Top $_{0}$ & Categoria dos espaços baseados e aplicações contínuas baseadas \\
\hline HTop & $\begin{array}{l}\text { Categoria dos espaços topológicos e das classes de equivalência } \\
\text { de aplicações homotópicas }\end{array}$ \\
\hline$\cong$ & Homeomorfismo \\
\hline$\sim$ & Homotopia \\
\hline$\approx$ & Isomorfismo \\
\hline$\wedge$ & Produto smash \\
\hline$V$ & Produto wedge \\
\hline$\Sigma$ & Suspensão \\
\hline$\Gamma$ & Função levantamento \\
\hline$H$ & Aplicação de Hopf \\
\hline$m(X, Y)$ & $\begin{array}{l}\text { Espaço das aplicações contínuas } f: X \rightarrow Y \text { com a } \\
\text { topologia compacto-aberta }\end{array}$ \\
\hline$m_{0}(X, Y)$ & $\begin{array}{l}\text { Espaço das aplicações contínuas baseadas } f: X \rightarrow Y \\
\text { com a topologia compacto-aberta }\end{array}$ \\
\hline$B^{I}$ & $=m(I, B)$ Espaço de todos os caminhos em $B$ \\
\hline$P Z$ & $=m_{0}(I, B)$ Espaço de todos os caminhos baseados em $B$ \\
\hline$\Omega Y$ & Espaço dos laços associados a $\left(Y, y_{0}\right)$ \\
\hline$[X, Y]$ & Conjunto das classes de homotopias livres \\
\hline$[X, Y]_{0}$ & Conjunto das classes de homotopias baseadas \\
\hline$\pi_{n}\left(Y, y_{0}\right)$ & $n$-ésimo grupo de homotopia de $\left(Y, y_{0}\right)$ \\
\hline$\pi_{n}^{p}$ & Componente $p$-primária do $n$-ésimo grupo de homotopia \\
\hline$X \sqcap Y$ & Espaço pull back \\
\hline$X \sqcup Y$ & Espaço push out \\
\hline $\operatorname{Princ}_{G}(B)$ & Conjunto do $G$-fibrados principais sobre o espaço $B$ \\
\hline & Grupo de gauge do fibrado $p$ \\
\hline 0 & Grupo de gauge do fibrado $p$ classificado pela aplicação $f$ \\
\hline
\end{tabular}




\section{Índice Remissivo}

$G$-ação principal, 19

$G$-aplicação, 19

$G$-espaço, 18

localmente trivial, 20

principal, 19

$G$-fibrado, 23

principal, 22

principal enumerável, 30

principal trivial, 26

universal, 31

$G$-homeomorfismo, 19

$k$-espaço, 43

$n$-fibrado universal, 31

push out, 6

pull back, 6

ação

efetiva, 18

própria, 19

transitiva, 18

aplicação

$G$-equivariante, 19

fibrada, 27

quociente induzida, 27

retração, 8

aplicações

adjuntas, 4

homotópicas, 4

homotópicas baseadas, 5

classes de homotopias

baseadas, 5

livres, 5

co-fibra, 11

co-fibração, 8

mapping cone, 9

composição secundária, 54

cone, 8 espaço

base, 22

baseado mapping track, 12

classificante, 31

das órbitas, 18

dos caminhos, 7

dos caminhos baseados, 9

dos laços, 4

homogêneo, 18

retrato, 8

total, 22

mapping path, 12

mapping track, 12

pull back, 6

push out, 6

fibra, 8

de homotopia, 12

fibração, 8

mapping path, 11

mapping track, 11

fibrado

induzido, 27

linha, 23

função

avaliação, 3

levantamento, 7

própria, 19

transitiva, 23

translação, 19

grupo

de gauge, 31

de gauge local, 34

de homotopia, 5

de isotropia, 19

de tranformação, 18

topológico, 17 
homotopia, 4

lei de composição

horizontal, 7

vertical, 7

lei exponencial, 4

produto

de fibras, 28

de Whitehead, 48

smash, 4

wedge, 4

retrato de deformação forte, 8

seqüência exata

de uma co-fibração, 11

longa de espaços e aplicações, 9

subconjunto compactamente fechado, 43

suspensão, 4

translação à direita, 18

trivialização, 22 